Journal of

Nutrigenetics

Nutrigenomics
J Nutrigenet Nutrigenomics 2012;5:171-302 DOI: 10.1159/000343955

\title{
6th Congress of the International Society of Nutrigenetics/ Nutrigenomics (ISNN)
}

\section{Abstracts}

\author{
November 18-21, 2012, \\ São Paulo, Brazil
}

\author{
Guest Editors \\ Rui Curi, São Paulo \\ Franco Lajolo, São Paulo \\ Thomas Ong, São Paulo \\ Raffaele De Caterina, Chieti/Pisa
}




\section{Journal of \\ Nutrigenetics \\ Nutrigenomics}

\section{President}

Raffaele De Caterina (Chieti, Italy)

\section{Past President}

Artemis P. Simopoulos (Washington, D.C., USA)

\section{Secretary/Treasurer}

Jing X. Kang (Charlestown, USA)

\section{Members}

Michel de Logeril (Grenoble, France)

Lynnette R. Ferguson (Auckland, New Zealand)

Serge Ferrari (Geneva, Switzerland)

Ronald Krauss (Oakland, USA)

Renato Mariani-Constantini (Chieti, Italy)

Alfredo Martínez (Pamplona, Spain)

John A. Milner (Rockville, USA)

Sergio Muntoni (Cagliary, Italy)

Marjanne Senekal (Cape Town, South Africa)

Charles N. Serhan (Boston, USA)

Gérard Siest (Nancy, France)

Antonio Velázquez (Mexico City, Mexico)

Nikos Yiannakouris (Athens, Greece) 


\section{Journal of \\ Nutrigenetics \\ Nutrigenomics}

\section{Welcome Address}

\section{Dear Colleagues,}

It is with great pleasure that we welcome you to this 6th Congress of the International Society of Nutrigenetics/Nutrigenomics (ISNN), held in the City of São Paulo, Brazil (November 18th$21 \mathrm{st}, 2012$ ). This edition of the congress is being organized by the ISNN together with its Brazilian partners and co-organizers: the University of São Paulo (USP) and the Brazilian Food and Nutrition Society (SBAN).

Nutrigenomics/Nutrigenetics represent a post-human genome scientific discipline that has received increasing awareness by the academy, health professionals, food industry and the general public. As a multidisciplinary field, it adopts an integrative view towards the understanding on how gene-nutrient interactions manifest and profoundly influence health and disease states. Because non-communicable diseases represent major global public health problems and are deeply influenced by diet and genes, the 6th ISNN Congress scientific program will cover the impact of nutrients on gene expression (nutrigenomics), as well as the impact of genetic variation on dietary response (nutrigenetics), in the context of cardiovascular diseases, obesity, diabetes, cancer, inflammation, metabolic control, human development, and exercise and muscle function. The translation of nutrigenetic/nutrigenomic knowledge into health solutions will be further addressed.

This congress will provide a special forum for junior scientists to present and discuss their research accomplishments, through the Young Investigator Award session. Moreover, because nutrigenetics/nutrigenomics is an emerging scientific discipline, the 6th ISNN Congress will also offer as a pre-congress activity the Nutritional Genomics Introductory Workshop, that is being organized by ISNN, SBAN and the Dietitians in Integrative and Functional Medicine Dietetic Practice Group of the American Academy of Nutrition and Dietetics.

We would also like to take this opportunity to acknowledge the fundamental support of the Brazilian Funding Agencies (FAPESP, CNPq and CAPES), Industry sponsors, and Institutional partners. Moreover, we would also like to thank Meeting Eventos for the efficient management of all logistical aspects of the Congress and to Dr. Jing Kang and the Editorial team of the Journal of Nutrigenetics and Nutrigenomics at Karger for all the assistance with this issue with the Congress abstracts. Special thanks is also dedicated to the Organizing, Scientific and Divulgation Committees involved in the congress planning and to all of our colleagues that accepted to participate as speakers and co-chairs.

We hope that you experience an unique meeting, from both the scientific and social perspectives. We also expect this Congress to represent a dynamic forum for sharing ideas and views on Nutrigenetics/Nutrigenomics, during these 4 days in São Paulo, a city you will find a vibrant and cosmopolitan destination great for both science and leisure.

Thank you all for taking part in the 6th Congress of the International Society of Nutrigenetics/ Nutrigenomics!

Warmest regards,

\author{
Rui Curi \\ Franco Lajolo \\ Co-Presidents of the Congress
}

Thomas Ong

President of the Organizing Committee

Raffaele De Caterina

President of the ISNN

\section{KARGER \\ Fax +4161306 1234 \\ E-Mail karger@karger.ch}

www.karger.com

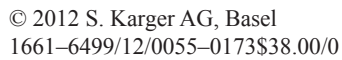

(C) 2012 S. Karger AG, Basel

$1661-6499 / 12 / 0055-0173 \$ 38.00 / 0$

Accessible online at:

www.karger.com/jnn 


\section{Journal of \\ Nutrigenetics \\ Nutrigenomics}

\section{Congress Co-Presidents}

Rui Curi, Institute of Biomedical Sciences, University of São Paulo, Brazil

Franco Maria Lajolo, Faculty of Pharmaceutical Sciences,

University of São Paulo, Brazil

\section{Congress Co-Chairs}

Raffaele De Caterina, President of the International Society of Nutrigenetics and Nutrigenomics

Eder Quintão, Faculty of Medicine, University of São Paulo, Brazil Mário Hiroyuki Hirata, Faculty of Pharmaceutical Sciences,

University of São Paulo, Brazil

Silvia Cozzolino, Faculty of Pharmaceutical Sciences,

University of Sao Paulo, Brazil

\section{Scientific Committee}

André Marette, Laval University, Canada

Antonio Velazquez, Autonomous National University of Mexico

Artemis P. Simopoulos, The Center for Genetics, Nutrition and

Health, USA

Dan Waitzberg, Faculty of Medicine, University of São Paulo, Brazil

Dulcinéia Saes Parra Abdalla, Faculty of Pharmaceutical Sciences,

University of Sao Paulo, Brazil

Helio Vannucchi, Faculty of Medicine of Ribeirão Preto,

University of São Paulo, Brazil

Jing Kang, Harvard Medical School, USA

Jorge Mancini, Faculty of Pharmaceutical Sciences,

University of São Paulo, Brazil

J. Alfredo Martinez, University of Navarra, Spain

John Milner, USDA/ARS, USA

Lynnette R. Ferguson, University of Auckland, New Zealand

Louis Pérusse, Laval University, Canada

Sergio Paiva, Faculty of Medical Sciences, São Paulo State

University at Botucatu, Brazil

Silvia Cozzolino, Faculty of Pharmaceutical Sciences,

University of Sao Paulo, Brazil

Susan Ozanne, University of Cambridge, United Kingdom

\section{Organizing Committee}

Thomas Ong (President), Faculty of Pharmaceutical Sciences, University of São Paulo, Brazil

Alice Cristina Rodrigues, Institute of Biomedical Sciences, University of São Paulo, Brazil

Ângelo Rafael Carpinelli, Institute of Biomedical Sciences, University of São Paulo, Brazil

Hosana Gomes Rodrigues, Institute of Biomedical Sciences, University of São Paulo, Brazil

Julio Tirapegui Toledo, Faculty of Pharmaceutical Sciences,

University of São Paulo, Brazil

Lucia Regina Ribeiro, State University of São Paulo, Brazil

Maria Fernanda Cury Boaventura, Cruzeiro do Sul University,

São Paulo, Brazil

Marcelo Rogero, Public Health Faculty, University of São Paulo, Brazil

Marco Aurelio Vinolo, Institute of Biomedical Sciences,

University of São Paulo, Brazil

Renata Gorjão, Cruzeiro do Sul University, São Paulo, Brazil

Sandro Massao Hirabara, Cruzeiro do Sul University, São Paulo,

Brazil

Tania Cristina Pithon Curi, Cruzeiro do Sul University, Brazil

William Festuccia, Institute of Biomedical Sciences,

University of São Paulo, Brazil

\section{Divulgation Committee}

Alexandre Rodrigues Lobo, Divulgation Committee of the Brazilian Food and Nutrition Society, Brazil

Carla Soraya Maia, State University of Ceará, Brazil

Carol Leandro, Federal University of Pernambuco, Brazil

Claudio Bernal, Universidad Nacional del Litoral, Argentina

Cristiane Cominetti, Federal University of Goiás, Brazil

Dilina Marreiro, Federal University of Piauí, Brazil

Eliane Fialho, Federal University of Rio de Janeiro, Brazil

Everardo Magalhães Carneiro, University of Campinas, Brazil

Helen Hermana M. Hermsdorff, Federal University of Viçosa, Brazil

Jussara Gazzola, Federal University of Santa Catarina, Brazil

Luiz Antonio Salazar, Universidad de La Frontera, Chile

Neuza Brunoro, Vila Velha Federal University, Brazil

Roberto Barbosa Bazotte, State University of Maringá, Brazil 


\section{(4) \\ Nutrigenetics \\ Nutrigenomics}

\section{Sunday, November 18}

\begin{tabular}{|c|c|}
\hline $10: 00-17: 30$ & Registration \\
\hline $12: 30-17: 00$ & $\begin{array}{l}\text { Promoted by: } \\
\text { - International Society of Nutrigenetics/Nutrigenomics (ISNN) } \\
\text { - Brazilian Food and Nutrition Society (SBAN) } \\
\text { - Dietitians in Integrative and Functional Medicine DPG (Dietetic Practice Group) of the American Academy of Nutrition } \\
\text { and Dietetics } \\
\text { Coordinators: } \\
\text { Thomas Ong, University of São Paulo, Brazil } \\
\text { Colleen Fogarty Draper, American Academy of Nutrition and Dietetics, USA }\end{array}$ \\
\hline $12: 30-12: 45$ & $\begin{array}{l}\text { Introduction and Welcome } \\
\text { Thomas Ong, Brazil }\end{array}$ \\
\hline $12: 45-13: 15$ & $\begin{array}{l}\text { Genetics 101: Genetics and Basic Aspects of Molecular Biology } \\
\text { Colleen Fogarty Draper, USA }\end{array}$ \\
\hline $13: 15-13: 45$ & $\begin{array}{l}\text { Nutritional Genomics: Dietary Modulation of Gene Expression: Implications for Health and Disease } \\
\text { Chandan Prasad, Texas Woman's University, USA }\end{array}$ \\
\hline $14: 30-15: 00$ & $\begin{array}{l}\text { Nutrigenetics and Implications for Dietetics Practice } \\
\text { Colleen Fogarty Draper, USA }\end{array}$ \\
\hline $15: 00-15: 30$ & $\begin{array}{l}\text { A New strategy to Analyze Gene-Nutrient Interaction } \\
\text { Jaqueline Pontes Monteiro, University of São Paulo, Brazil }\end{array}$ \\
\hline $15: 30-15: 45$ & Break \\
\hline $15: 45-16: 15$ & $\begin{array}{l}\text { Nomenclature and Databases in Nutrigenetics } \\
\text { Ron Martin, Nutrigenetics Unlimited, Inc, USA }\end{array}$ \\
\hline $16: 15-16: 30$ & $\begin{array}{l}\text { Nutritional Genomics Education: Teaching Nutritional Genomics to Dietetics Students } \\
\text { Chandan Prasad, USA }\end{array}$ \\
\hline $\begin{array}{l}16: 30-17: 00 \\
17: 30-18: 30\end{array}$ & $\begin{array}{l}\text { Roundtable Discussion } \\
\text { Opening Ceremony }\end{array}$ \\
\hline
\end{tabular}

Key Note Presentation

Co-Chairs:

Raffaele De Catarina, University of Chieti, Italy

Rui Curi, University of São Paulo, Brazil

$\begin{array}{ll}\text { 18:30-19:30 } & \text { State of the Art on Nutrigenetics and Nutrigenomics } \\ \text { Artemis P. Simopoulos, The Center for Genetics, Nutrition and Health, USA }\end{array}$

\section{Monday, November 19}

08:40-10:35

Session 1: Nutrients and Cardiovascular Diseases

Co-Chairs:

Éder Quintão, University of São Paulo, Brazil

J. Alfredo Martinez, University of Navarra, Spain

08:40-09:00

Genetic Determinants of Blood Pressure Responses to Caffeine Drinking

Raffaele De Caterina, University of Chieti, Italy

09:00-09:20 Gene Variants Influencing Cardiovascular Risk Factors in an Admixted Population - Brazilians

Jose Eduardo Krieger, University of São Paulo, Brazil 
09:20-09:40 Nutrigenetic Studies of Cardiovascular and Metabolic Diseases Hooman Allayee, University of Southern California, USA

09:40-10:00 The Impact of Plant Sterols on Lipids and Inflammatory Markers Raul Dias dos Santos Filho, University of São Paulo, Brazil

10:00-10:20 Genetic Markers of Obesity and Lowering-Body Weight Response to Dietary Interventions Rosario Dominguez Crespo Hirata, University of São Paulo, Brazil

10:20-10:35 Discussion

10:35-11:00

\section{Coffee-Break}

11:00-12:35

Session 2: Gene-Nutrient Interaction in the Control of Leukocyte Function

Co-Chairs:

Francisco Soriano, University of São Paulo, Brazil

Susan Ozanne, Cambridge University, UK

11:00-11:20 Fatty Acids, Inflammation and Wound Healing

Rui Curi, University of São Paulo, Brazil

11:20-11:40 The Role of the Microbiota and Its Metabolites in Controlling Acute Inflammatory Responses

Mauro Teixeira, Federal University of Minas Gerais, Brazil

11:40-12:00 Pro-Resolving n-3 Mediators Stimulate Resolution of Inflammation and Tissue Regeneration

Nan Chiang, Harvard University, USA

12:00-12:20 Integration of Genomics, Proteomics, \& Metabolomics of Lipid Metabolism

Edward Dennis, University of California at San Diego, USA

12:20-12:35

Discussion

12:35-14:00 Lunch

14:00-15:35 Session 3: Epigenetics in Human Development and Chronic Diseases

Co-Chairs:

Ana Lydia Sawaya, Federal University of São Paulo, Brazil

Francisco Bolaños-Jiménez, University of Nantes, France

14:00-14:20 Nutritionally Programmed Influences on Gene Expression- a Mechanism Underlying the Developmental Origins of Health and Disease

Susan Ozanne, Cambridge University, UK

14:20-14:40 The Epigenome as a Promising Target for Breast Cancer Prevention with Bioactive Food Compounds

Thomas Ong, University of São Paulo, Brazil

14:40-15:00 Epigenetics as a Mediator of the Impact of Nutrition on Bowel Cancer Development

John Mathers, University of Newcastle, UK

15:00-15:20 Diet and MicroRNA Regulation of Inflammatory Bowel Disease

Lynnette R. Ferguson, University of Auckland, New Zealand

15:20-15:35 Discussion

15:35-16:00 Coffee-Break

16:00-18:00 Session 4: Gene-Nutrient Interaction in Metabolic Control

Co-Chairs:

Mauro Teixeira, Federal University of Minas Gerais

Louis Pérusse, Laval University, Canada

16:00-16:20 Transcriptional Program Associated with a Dietary Life-Style Intervention in LDR-/- Mice

Marijana Radonjic, TNO, The Netherlands

16:20-16:40 Regulation of Lipogenic Gene Expression Profile and Adiposity by the Nutrient Sensors mTOR e PPAR

William Festuccia, University of São Paulo, Brazil

16:40-17:00 Energy-Sensing Changes Induced by Perinatal Nutrition and their Implications for the Development of Metabolic Disorders

Francisco Bolaños-Jiménez, University of Nantes, France

17:00-17:20 Hypothalamic Dysfunction in Obesity

Lício A. Velloso, State University of Campinas, Brazil

17:20-17:40 Control of the Kidney Inflammation by Short Chain Fatty Acid

Neils Camara, University of São Paulo, Brazil

17:40-18:00

Discussion 


\section{Tuesday, November 20}

08:40-10:35 Session 5: Gene-Nutrient Interaction in Obesity and Diabetes

Co-Chairs:

Antonio Velazquez, Universidad Autónoma de Mexico, Mexico

Jing Kang, Harvard University, USA

08:40-09:00 Gene-Nutrient Interaction in Obesity and Diabetes

Hannelore Daniel, Technische Universität München, Germany

09:00-09:20 Classical and Novel Gene Targets of Long-Chain Omega3 Fatty Acids and their Role in the Prevention of Obesity-Linked

Diabetes

André Marette, Laval University, Canada

09:20-09:40 Gut Microbiota is a Key Modulator of Insulin Resistance in TLR 2 Knockout Mice

Mario Saad, State University of Campinas, Brazil

09:40-10:00 Dietary Interventions in Obese People as Affected by the Genotype: Nutrigenetic Approaches

J. Alfredo Martinez, University of Navarra, Spain

10:00-10:20 Genetic Determinants of Obesity and Weight Changes in Response to Obesity Treatments

Louis Pérusse, Laval University, Canada

10:20-10:35 Discussion

10:35-11:00 Coffee-Break

$\begin{aligned} \text { 11:00-12:35 } & \text { Session 6: Gene-Nutrient Interaction in Diet and Cancer } \\ & \text { Co-chairs: } \\ & \text { Dan Waitzberg, University of São Paulo, Brazil } \\ & \text { Lynnette R. Ferguson, University of Auckland, New Zealand }\end{aligned}$

11:00-11:20 Genomic-Nutrient Interactions in Cancer Prevention: Defining Molecular Targets for Bioactive Food Components John A. Milner, USDA/ARS, USA

11:20-11:40 Fatty Acids, Eicosanoids and Brain Tumours

Alison Colquhoun, University of São Paulo, Brazil

11:40-12:00 Omega-6/Omega-3 PUFA Ratio and Tumorigenesis

Jing X. Kang, Harvard University, USA

12:00-12:20 Structured Lipids from Tributyrin, a Dietary Histone Deacetylase Inhibitor, and Flax Seed Oil: Cellular and Molecular Effects During Hepatocarcinogenesis Chemoprevention

Fernando Salvador Moreno, University of São Paulo, Brazil

12:20-12:35 Discussion

12:35-14:00 Lunch

14:00-15:35 Session 7: Translation of Nutrigenetic/Nutrigenomic Knowledge

Co-Chairs:

Franco M. Lajolo, University of São Paulo, Brazil

Raffaele De Caterina, University of Chieti, Italy

14:00-14:20 Nutrigenomics Research: Personalized Nutrition and Medicine Jim Kaput, Nestlé Institute of Health Sciences, Switzerland

14:20-14:40 Caenorhabditis Elegans as a Predictive Model for a Nutrigenomics Approach to Identify New Mechanistic Insights on Potential Probiotic Bacteria

Gianfranco Grompone, Pasteur Institut de Montevideo, Uruguay

14:40-15:00 Practical Application of Nutrigenomics for the Development of an Oral Cosmetic Anti-Wrinkle Supplement

Robin van den Berg, Unilever Research\&Development, Netherlands

15:00-15:20 "From Micro-Array to Daily Meal Tray"

Jean Michel Antoine, Danone Research, France

15:20-15:35 Discussion

15:35-16:00 Coffee-Break

16:00-18:00 Poster Visiting and Discussion Session 


\section{Wednesday, November 21}

08:40-10:35 Session 8: Young Investigator Award

Co-Chairs:

Lucia Ribeiro, State University of São Paulo, Brazil

Artemis Simopoulos, The Center for Genetics, Nutrition and Health, USA

08:40-08:50 Regulation of Lipid Metabolism Transcription Factors by Polyunsaturated Fatty Acids on the Postpartum Period of

Successive Generations in Rats

Carolina Bespalhok Jacometo, Federal University of Pelotas, Brazil

08:50-09:00 The IFAT-1 Mouse Model: Studying Temporal and Tissue Specific Effects of n-3 Polyunsaturated Fatty Acid Enrichment Shannon E. Clarke, University of Guelph/MGH Harvard Medical School, Canada/USA

09:00-09:10 Involvement of Glomerular Micro-RNAs Expression on Epithelial-to-Mesenchymal Transition in Adult Maternal Low Protein Diet Programmed Rats

Letícia Barros Sene, State University of São Paulo Júlio de Mesquita, Brazil

09:10-09:20 Methylation of IL6 Gene Promoter in Peripheral White Blood Cells is Associated with Truncal Fat and Plasma Free Fatty Acids in Young Women Helen Hermana M. Hermsdorff, Federal University of Viçosa/University of Navarra, Brazil/Spain

09:20-09:30 FTO T/A, PPARGAMMA PRO12ALA But Not APOA1-75 Polymorphisms Are Associated with Better Response to Lifestyle Intervention in Brazilians at High Cardiometabolic Risk

Maíra Ladeia R. Curti, University of São Paulo, Brazil

09:30-09:40 Moderate Alcohol Consumption Alters Hepatic Metabolism-Related Markers in Rats with Pre-existing NASH

Andre Ferreira Nascimento, UNESP/TUFTS University, USA

09:40-09:50 Influence of Yerba Mate (Ilex Paraguariensis) in Insulin Signaling Pathway

Demetrius Paiva Arçari, UNICAMP/USF, Brazil

09:50-10:00 Carbon Metabolism Genomic Changes Are Caused by Reduced ATP Production in Biotin Starvation

Estefania Ochoa-Ruiz, Universidad Nacional Autonoma de Mexico, Mexico

10:00-10:10 Docosahexaenoic Acid Regulates Genes Related with Lipid Metabolism Differently in Normal and Breast Cancer Cell Line: a Microarray Approach

Danielle Fontes Almeida, Faculty of Medicine of the University of São Paulo, Brazil

10:10-10:20 Stimulation of Muscle Growth and Carbohydrate Metabolism Genes by Dietary Brassinosteroids

Debora Esposito, North Carolina State University, USA

10:20-10:35 Discussion

10:35-11:00 Coffee-Break

11:00-12:35 Session 9: Gene-Nutrient Interaction in Exercise, Chronic Diseases and Skeletal Muscle Function

Co-chairs:

Hannelore Daniel, Technische Universität München, Germany

Julio Orlando Tirapegui Toledo, University of São Paulo, Brazil

11:00-11:20 Molecular Mechanisms Involved in Skeletal Muscle Atrophy

Anselmo Moriscot, University of São Paulo, Brazil

11:20-11:40 Catalase Overexpression and Mitochondrial Function in Insulin Resistant Muscle Cells Leonardo dos Reis Silveira, University of São Paulo, Brazil

11:40-12:00 Genomics in Sports: The Athletes of the Future Project

João Bosco Pesquero, Federal University of São Paulo, Brazil

12:00-12:10 Discussion

12:10-13:00 Session 10: Oral Abstract Presentations

12:10-12:30 Bitter Taste Receptor Polymorphisms and Human Aging

Roberto Barale, University of Pisa, Italy

12:30-12:50 Genetic Polymorphism of Homocysteine Metabolic Genes and Susceptibility of Type 2 Diabetes in Chinese

Duo Li, Zhejiang University China

12:50-13:00 Discussion

13:00-14:00 Lunch

14:00-15:15 Session 11: Round Table - Perspectives and Challenges in Nutrigenetics and Nutrigenomics

Raffaele De Caterina, University of Chieti, Italy

Franco M. Lajolo, University of São Paulo, Brazil

Louis Pérusse, Laval University, Canada

J. Alfredo Martinez, University of Pamplona, Spain

John A. Milner, USDAVARS, USA

Artemis P. Simopoulos, The Center for Genetics, Nutrition and Health, USA

15:15-15:30 Closing Session 


\section{Nutritional Genomics Introductory Workshop}

\section{1 \\ Dietary Modulation of Gene Expression: Implications for Health and Disease}

\author{
Chandan Prasad \\ Professor \& Chair, Department of Nutrition and Food \\ Sciences, Texas Woman's University, Denton, TX 76204
}

"Let thy food be thy medicine and thy medicine be thy food." - Hippocrates (460-377 B.C.)

It was not until the early naturalistic era (circa $400 \mathrm{BC}$ ) that diet was recognized as an important element in disease prevention and treatment. This is reflected by a quote from Hippocrates, the great physician-philosopher, who lived during the early part of the era. Since then, we have made great progresses in our understanding of nutrition and its role in disease as well as maintenance of good health. However, it was not until some 25 years ago that we could explain why some individuals who consume high fat diet, show no evidence of atherosclerotic disease like most others? The answer to this question lies in our inherent genetic differences that make us respond differently to different dietary components. The completion of the sequencing of the human genome in 2001 has not only provided tools but also ignited interest in our search for role genetics in handling of nutrients and vice versa. In today's talk I am going to summarize how different components of the food that we consume affect gene expression. Examples will come from modulation of gene expression by macronutrients (fats, carbohydrates and proteins), micronutrients (vitamins and minerals) and other food components (flavonoids and xenobiotics). Time permitting, I will also touch upon how food components can also affect expression and function of essential proteins.

\section{2}

\section{Nutritional Epigenomics}

Thomas Ong

Faculty of Pharmaceutical Sciences, Food and Nutrition Research Center, University of São Paulo, Brazil

Epigenetics refers to heritable changes in gene expression that are not accompanied by alterations in DNA sequence. The main epigenetic processes are DNA methylation, histones post-translational mod- ifications and microRNAs. They modulate gene expression by altering chromatin architecture. Increasing evidence shows that nutrients influence these epigenetic processes. 1-carbon metabolism, in which folate and vitamin B12 are key components, provides the methyl groups for cytosine and histone methylation reactions. Several other food components including green tea catechins, garlic allylcompounds, retinoids, fatty acids and selenium have been shown to modulate the activity of enzymes that integrate the epigenetic machinery, including DNA methyltransferases and histone deacetylases and acetyltransferases. More recently, nutritional regulation of microRNAs has been reported. Dietary modulation of the epigenome expands the complexity of gene-nutrient interactions. At the molecular level, non-communicable diseases including not only cancer, but also obesity, diabetes, cardiovascular and neurodegenerative diseases display profound epigenetic deregulation. Inadequate nutritional modulation of DNA methylation, histone acetylation and methylation and microRNA expression during early life, in which the epigenome is especially plastic, could be associated with the developmental origin of health and disease (DOHaD). Thus a deeper understanding of the mechanisms whereby nutrients exert such influences will be fundamental for the definition of strategies for health promotion and disease prevention, that should consider a life course approach probably starting early in life.

03

\section{Nutrigenetics and Implications for Dietetics Practice}

Colleen Draper

American Academy of Nutrition and Dietetics, Nestlé Institute of Health Sciences, Lausanne, Switzerland

Nutrigenetics is focused on the impact alterations in our genes have on our potential health trajectory, which is strongly influenced by food, nutrition, stress, and toxins. In order to understand an individual's health trajectory of resilience and adaptability, we need to assess multiple components of the health equation. One essential component is genetic susceptibility. Although we know gene polymorphisms, such as single gene polymorphisms (SNPs) only contribute $1 \%$ to our knowledge of heritability, and the large number of variants (including approximately 2 million SNPs) identified through genome wide association studies on large numbers of people (as much as 250,000 in some studies) so far explain only $5-10 \%$ of heritability for common chronic health issues, such as dyslipidemia, obesity, and diabetes, this offers us a place to start. Ultimately, the outcomes of this equation need to be measured and monitored, through gene expression analyses (mRNA microarrays), proteomic and metabolomic analyses. For example, urinary metabolite testing is already used by early adopters in nutrition practice to conduct a func- 
tional nutrition assessment that identifies early phase markers of metabolic dysfunction that, if left unchecked, will eventually result in a loss of health plasticity and disease will ensue. Nutritional genetic susceptibility testing has been available commercially, although sporadically, for the last decade. It is important to know the limitations of this information. This includes: missing disease risk heritability, which has not been fully explained due to the need for a better understanding of epistasis (gene $\mathrm{x}$ gene interactions - including the newly recognized impact of copy number variants), impact of rare variants, epigenomics interactions, and environmental interactions not yet identified.

\section{4}

\section{A New Strategy to Analyze Gene-Nutrient Interaction}

Jacqueline Pontes Monteiro ${ }^{1 *}$, Carolyn Wise ${ }^{2}$, Anna Williams², Bridgett Green², Lisa Pence3, Melissa Morine 4 , Marco Scotti ${ }^{4}$, Margaret Bogle, Beverly McCabe-Sellers ${ }^{5}$, Catherine Champagne, Jerome Turner ${ }^{7}$, Beatrice Shelby ${ }^{7}$, Corrado Priami ${ }^{4}$, Richard Beger ${ }^{3}$, Jim Kaput ${ }^{8 *}$

${ }^{1}$ Department of Pediatrics, Faculty of Medicine. Faculty of Nutrition and Metabolism, University of Sao Paulo Ribeirao Preto. SP, Brazil; '2Division of Personalized Nutrition and Medicine, National Center for Toxicological Research, Food and Drug Administration. Jefferson, AR, USA; ${ }^{3}$ Division of Systems Biology, National Center for Toxicological Research, Food and Drug Administration. Jefferson, AR, USA; ${ }^{4}$ The Microsoft Research - University of Trento Centre for Computational and Systems Biology (COSBI). Trento, Italy; ${ }^{5}$ Delta Obesity Prevention Research Unit. United States Department of Agriculture Agricultural Research Service. Little Rock, AR. USA; ${ }^{6}$ Dietary Assessment and Nutrition Counseling. Pennington Biomedical Research Center. Baton Rouge, LA. USA; ${ }^{7}$ Boys, Girls, and Adults Community Development Center. Marvell, AR. USA; ${ }^{8}$ Clinical Translation Unit. Nestle Institute of Health Sciences. Lausanne, Switzerland Affiliated with Division of Personalized Nutrition and Medicine. NCTR/FDA. Jefferson, AR. USA for portions of this study

The challenges with analyzing gene-nutrient interactions include: genetic heterogeneity in humans: the complexity of environmental factors, with particular attention to dietary intake differences, the resulting diverse physiologies that produce the same apparent disease and, to provide reliable and predictable personalized dietary recommendations for specific health outcomes. Our novel experimental approach is (i) to use cross over intervention designs for nutrients with small effect sizes, (ii) use deep phenotyping and genotyping methods (iii) identify patterns with and between different data types using conventional and novel statistical methods, (iv) use dietary and lifestyle information to interpret results of group and level individual analyses, and (v) make recommendations for diet changes. This strategy was developed and tested in a 2-year intervention study of 61 children ages 6 through 14 (23 boys and 38 girls). Plasma levels of homocysteine (HCys), SAM, SAH, pyridoxal, pyridoxine, thiamin, riboflavin, vitamin $\mathrm{A}$, vitamin $\mathrm{E}$ and vitamin $\mathrm{D}$ were analyzed in samples taken before, at the end, and one month after a whole food intervention trial. Discovery methods identified two SAM/SAH clusters, one with a mean SAM/SAH of $1.81(\mathrm{C} 1)$ and the other $(\mathrm{C} 2)$ with a mean of $0.81(\mathrm{p}=<10.4)$. Generalized Linear Mixed Effects analyses discovered patterns of 182 SNPs in 81 genes that differed significantly between $\mathrm{C} 1$ and $\mathrm{C} 2$. The average gene methylation differences were similar between $\mathrm{C} 1$ and $\mathrm{C} 2$. The SAM/SAH ratio in children with genotype A was sensitive to decreased nutrient intake values. $\mathrm{SAM} / \mathrm{SAH}$ ratio was associated with nutritional status and may be a marker of poor nutrition in this population. Genetic patterns associated with response to the intervention were found and analyzed through a systems biology approach. Replicating and validating nutritional genomics studies on the individual level are a priority before personalized nutrition can be considered a valid approach to improving human health.

\section{5 \\ Nomenclature and Databases in Nutrigenetics}

Ron L. Martin

President, Nutrigenetics Unlimited, Inc., Fullerton, California, USA

Nutrition is the most universal example of gene-environment interaction since its unavoidable impact begins even before birth and continues throughout life. Gene-environment interactions are profound because they affect our physical health, emotions, behaviors, and sense of well-being. Although automated tools for clinical decision support are constantly being pursued, it is unlikely that automation will ever completely replace the need for individualized attention from professionals. In order to effectively utilize the "evidence base" for either nutrition or medicine, one of the most basic needs is the ability to recognize within the literature the multiple synonyms for genes and gene variants used by different authors. Examples will be shown of common synonyms for genes and gene variants relevant to nutrition. For instance, a single nucleotide polymorphism (SNP) may sometimes be described by its nucleotide substitution (e.g., 677C-T for the MTHFR gene), or by its corresponding amino acid substitution (Ala222Val, or A222V), or by its reference SNP number (rs1801133). Resources for identifying synonyms for both genes and gene variants will be described, including the Human Gene Nomenclature database at www.genenames.org, or the OMIM database at www.ncbi.nlm.nih.gov/omim, or the MeSH Browser at www. nlm.nih.gov/mesh/MBrowser.html, or the SNP database at www.ncbi. nlm.nih.gov/sites/entrez?db $=$ Snp. Besides assisting with nomenclature, the database at www.nutrigenetics.net can also help identify exactly which genes and gene variants have been studied in connection with any given topic, including nutrients, health conditions, pharmaceuticals, and other risk modifiers. Valuable tools are already available for studying gene-environment interactions including nutrition, and for translating that information into clinical practice, as listed above with others to be described. Practical use of tools like 
these requires both education and engagement with researchers, health professionals, and members of the public - including educators, students, journalists, and companies.

\section{6}

\section{Teaching Nutritional Genomics to Dietetics Students}

Chandan Prasad

Professor \& Chair, Department of Nutrition and Food

Sciences, Texas Woman's University, Denton, TX 76204

Nutritional genomics offers a great promise to personal health management based on understanding of relationship between diet, gene expression and health outcomes. While the ideal healthcare provider for delivery of nutrigenomic education to public will be the primary care physician (general internist, family medicine physician, or pediatrician), this is unlikely to come to fruitions for many reasons. These include, a greater demand on physicians to see more patients in less time as well as lack of emphasis on training of physicians in the science of nutrition and nutrigenomics in the medical schools, or during residency training. With physicians out of picture at the moments, the responsibility of nutrigenomic education must fall on the shoulders of Registered Dietitian (RD). These RDs serve in hospitals, clinics, nursing homes, athletic facilities, schools and also as private practitioners as nutrition consultants. This highlights an urgent need for training dietitians in the science and practice of nutritional genomics. Our readiness to deliver nutrigenomics-based nutrition education has been slow due to complexity of gene-nutrient interaction and interplays between many disciplines such as genetics, nutrition, biostatistics, sociology, law and philosophy in the process. In this talk, I would like to present some key learning objectives that dietitians must master to be able to educate public with not necessarily a nutrition advice based on gene profiling, but also limitations of this technology.

\section{Keynote Presentation}

07

\section{State of the Art on Nutrigenetics and Nutrigenomics}

\author{
Artemis P. Simopoulos \\ The Center for Genetics, Nutrition and Health, \\ Washington, DC, USA
}

The health of the individual and the population in general results from the interactions between genetic and environmental factors. Diet (Nutrition) is an environmental factor of major importance, both in the prevention of chronic diseases, in normal development, and in the maintenance of homeostasis. Studies on genetic variants and their response to diet in health and disease (Nutrigenetics) as well as studies on the role of nutrients in gene expression (Nutrigenomics) will lead the way in the development of personalized nutrition, in the definition of a healthy diet, and development of Novel Foods. To continue in its role as an integrator of genomic and environmental processes, Nutritional science is adjusting its focus to include the microstructure of the genome, the metabolome, the proteome, the epigenome, metagenomics, etc. Ultimately we will be able to understand nutritional metabolism at levels of discrimination sufficient to permit individual dietary prescription. In contemplating how genetics and Nutrigenetics/Nutrigenomics will look in the future, we can foresee that Genetics will not remain the exclusive prerogative of regional genetic centers. Instead, every physician will need to use genetic knowledge and combine it with appropriate dietary regimen, type and amount of physical activity, and if needed drugs. Expectations for the first quarter of the 21 st Century include: the identification of disease/ gene associations for many illnesses and function of genes, the expansion of Nutrigenetics/Nutrigenomics, Pharmacogenetics and Pharmacogenomics (treatment and prevention), the responsiveness to interventions (nutrients, drugs) will be predicted, since variation in the responses is often attributable to the genetic profile of the individual, the individualization of prescriptions, diets and life style modifications and/or drug treatment, and the development of gene based designer diets for coronary artery disease, hypertension, diabetes, arthritis, asthma, mental health, etc. Public health and regulatory processes will need to be established to define when genomic discoveries such as gene/nutrient disease associations are ready to be evaluated as potential tools to improve health screening and recommended dietary values.

\section{Nutrients and Cardiovascular Diseases}

\section{8 \\ Genetic Determinants of Blood Pressure Responses to Caffeine Drinking}

Raffaele De Caterina

Institute of Cardiology and Center of Excellence on Aging, "G. d'Annunzio" University, Chieti; "G. Monasterio"

Foundation, Pisa, Italy

Background: The widely observed between-subject variability in cardiovascular responses to coffee may have a genetic basis.

Objective: We evaluated acute blood pressure (BP) responses to caffeine and explored whether they are influenced by candidate gene variants affecting caffeine metabolism (for cytochrome P450 1A2), adenosine metabolism (for adenosine receptor and adenosine monophosphate deaminase), or catecholamine receptors. 
Methods: We recruited 110 male healthy habitual moderate coffee drinkers refraining from coffee for the day preceding the study. Each subject underwent ambulatory BP monitoring at 6 min intervals for $2 \mathrm{~h}$. Each participant was administered, in a double-blind design, $40 \mathrm{~mL}$ of either a decaffeinated coffee preparation plus $3 \mathrm{mg} / \mathrm{kg}$ caffeine ("caf") or the corresponding vehicle ("decaf"). The protocol was repeated $24 \mathrm{~h}$ later with the alternative preparation. Blood samples were collected for genetic, plasma caffeine and catecholamine evaluations.

Results: Compared with decaf, caf was associated with a significant increase in systolic and diastolic BP (SBP, DBP) (by $4 \pm 12$ $\mathrm{mmHg}$ for SBP and $3 \pm 10 \mathrm{mmHg}$ for DBP (mean $\pm \mathrm{SD}), \mathrm{P}<0.001$ for both). Plasma caffeine and adrenaline increased after caf, but not decaf. Out of 11 gene polymorphisms analyzed, a relationship between ADORA2A TT variant and $\triangle \mathrm{SBP}$ peak and between ADRA2B I variant and $\triangle \mathrm{SBP}$ mean and peak was observed: these variants were associated with increased SBP responses to caf.

Conclusions: Variability in the acute BP response to coffee may be partly explained by genetic polymorphisms of the adenosine A2A receptors and $\alpha_{2}$-adrenergic receptors.

\section{9 \\ Nutrigenetic Studies of Cardiovascular and Metabolic Diseases}

Hooman Allayee

Department of Preventive Medicine and Institute for Genetic Medicine, Keck School of Medicine, University of Southern California, Los Angeles, CA USA

Understanding the genetic basis of cardiovascular and metabolic diseases has been revolutionized by the ability to carry out association studies on a genome-wide basis. This approach has revealed dozens of novel genes for atherosclerosis, diabetes, obesity, as well as related quantitative phenotypes, but the identified susceptibility alleles still only explain $\sim 10 \%$ (or less) of the overall phenotypic variance. This can likely be attributed to several factors including: 1) relatively weak effects of the at-risk variants, 2) the low power to detect rare susceptibility alleles, and/or 3) that the effects are detectable only in the context of other genetic factors or an appropriate environmental exposure. In this regard, the interaction between genes and dietary factors is an area of cardiovascular and metabolic disease research that remains poorly understood. We have explored nutrigenetic studies of cardiovascular and metabolic traits using candidate gene approaches, with a particular focus on the leukotriene pathway. Leukotrienes (LTs) are mediators of inflammation synthesized from dietary polyunsaturated fatty acids (PUFAs) and have long been known to influence chronic allergic inflammatory conditions, such as asthma. However, studies from our group and others over the last several years support the concept that the LT pathway also plays a role in atherosclerosis and that genetic variation in this pathway interacts with dietary PUFAs to modulate disease risk. In other recent studies, we have similarly applied this approach to non-alcoholic fatty liver disease (NAFLD), which has dramatically risen in prevalence among minority populations, such as Hispanics. NAFLD also has a strong genetic component, with patatin-like phospholipase domain contain- ing 3 (PNPLA3) being the major susceptibility gene identified in adults across multiple ethnicities. We have validated the association of PNPLA3 with NAFLD in Hispanic children and observe that the genetic effects manifest as early as eight years of age. Furthermore, our data suggests that high dietary sugar exacerbates liver fat accumulation but only in subjects carrying the at-risk PNPLA3 allele. On overview of these studies will be presented with a discussion of how these results can be translated into novel and more effective treatment strategies for cardiovascular and metabolic diseases.

\section{0 \\ Genetic Markers of Obesity and Lowering- Body Weight Response to Dietary Interventions}

Rosario Dominguez Crespo Hirata

Faculty of Pharmaceutical Sciences, University of São Paulo, Brazil

Obesity is a wide world common disease that is caused by interactions of genetic, environmental and behavior factors resulting in excessive body fat accumulation. Obesity is a relevant risk factor for type 2 diabetes and cardiovascular disease. Gene candidate and genome scanning approaches have indicated various genetic polymorphisms potentially linked to obesity-related phenotypes. Common variants located within or near genes encoding proteins involved in energy homeostasis, such as leptin, leptin receptor, proopiomelanocortin, melanocortin 4 receptor and other genes, such as fat mass and obesity-associated (FTO), have been implicated on increased adiposity and susceptibility to obesity. Several studies have shown considerable variability on response to dietary interventions to reduce body weight and adiposity. This variability has been ascribed to interactions between genomic and environmental, and even psychosocial factors. This lecture aims to discuss results from some studies which investigated the relationship between obesity-related and other genes and response to dietary interventions for lowering body weight. Gene-diet interactions implicated in regulation of metabolic status, which are monitored in obese patients to reduce cardiovascular risk, will be also approached. 


\section{Gene-Nutrient Interaction in the Control of Leukocyte Function}

\section{1 \\ Fatty Acids, Inflammation and Wound Healing}

H.G. Rodrigues, M.A. Vinolo, J. Magdalon, E. Hatanaka, R. Curi

Department of Physiology and Biophysics, Institute of Biomedical Sciences, University of São Paulo, and Institute of Physical Activity and Sports, Cruzeiro do Sul University, São Paulo, Brazil

Vegetal oils with high content of oleic (OA) and linoleic (LA) acids have been used to treat patients with cutaneous wound but the mechanism involved remains unknown. The effect of topic application and oral administration of pure OA and LA on wound healing was investigated. A wound in the dorsal surface of adult rats was induced and OA and LA were topically administered. OA and LA increased the wound healing tissue mass, the number of neutrophils in the wounded area whereas the thickness of the necrotic cell layer edge around the wound was decreased. Oral administration of LA increased the influx of inflammatory cells, production of hydrogen peroxide, expression of CINC-2 $\alpha \beta$, and activation of the AP-1 whereas OA increased TNF- $\alpha$ concentration and NF- $\kappa$ B activation in the wound at 1 hour post wounding. Neutrophil migration in response to intraperitoneal injection of glycogen and in the air pouch assay was elevated by OA and LA. This effect was associated with enhancement of rolling, release of CINC-2 $\alpha \beta$, and L-selectin expression. At 24 hours post wounding, however, the fatty acids decreased the number of inflammatory cells and IL-1, IL-6, and MIP-3 content, and $\mathrm{NF}-\mathrm{\kappa B}$ activation in the wound. LA accelerated wound closure over a period of seven days. In cultured neutrophils, the fatty acids increased the production of VEGF-alpha, IL- $1 \beta$, and CINC-2 $\alpha \beta$. The wound healing involves inflammatory reaction, proliferation and maturation of newly formed tissue. OA and LA accelerate the inflammatory phase and so speed up the wound healing.

Research supported by FAPESP, CNPq, and CAPES.

\section{2 \\ The Role of the Microbiota and Its Metabolites in Controlling Acute Inflammatory Responses}

Mauro M. Teixeira, Caio T. Fagundes, A.M. Vieira, Daniele G. Souza

Immunopharmacology Laboratory, Institute of Biological Sciences, Universidade Federal de Minas Gerais, Belo Horizonte, MG, Brazil

The interaction between a host and its residing microorganisms has been found to be relevant to the development of the immune system. Recognition of microbial components by receptors of the host innate immune system triggers signals necessary for the development of the immune response. Such interaction is also relevant for the ability of higher organisms to mount an acute inflammatory response. Indeed, in the absence of microbiota (ie. In germ free - GF- mice), acute inflammatory responses are greatly decreased. This is not because GF animals cannot interact and respond to inflammatory stimulation. On the contrary, there is high production of anti-inflammatory mediators (including annexin-1, lipoxin A4 and IL-10) which actively prevent acute inflammation. Blockade of the these antiinflammatory molecules or restoration of the microbiota restore inflammatory responsiveness of GF mice. Because of their deficient ability to mount acute inflammatory responses, GF mice have deficient adaptive immune responses and capacity to deal with infections, such as by bacteria. Therefore, daily contact with bacteria fine tunes the immune system to deal with acute infections, at a cost of dampening anti-inflammatory pathways. Not all host-bacterial interactions facilitate inflammatory responses. Indeed, we have shown that decreased production of short chain free fatty acids (SCFAs) and absence of GPR43 (a receptor to which SCFAs bind) were associated with enhanced or prolonged tissue inflammation. On the other hand, administration of SCFAs or the use of certain prebiotics (higher fiber diet) and probiotics (Bifidobacterium longum) enhanced circulating levels of SCFAs and decreased intensity and duration of inflammatory responses. These effects were associated with activation of GPR43. Therefore, our studies clearly show that interactions with the microbiota control the inflammatory responsiveness of animals. Our studies also clearly show that dietetic modifications can favor growth of certain bacteria and production of metabolites that decrease inflammatory responsiveness via activation of the GPR43 receptor, therefore providing a molecular link between microbiota, diet and inflammatory response. 


\section{3 \\ Pro-Resolving N-3 Mediators Stimulate Resolution of Inflammation and Tissue Regeneration}

Nan Chiang, Charles N. Serhan

Center for Experimental Therapeutics and Reperfusion Injury, Department of Anesthesia, Perioperative and Pain Medicine, Harvard Institutes of Medicine, BWH and Harvard Medical School

The endogenous mechanisms involved in resolution of the acute inflammatory responses have provided new insights in host defense. Using a systems approach coupled with lipid mediator lipidomics, we uncovered several new families of potent bioactive lipid-derived mediators in resolving exudates. This presentation shall update new advances on the biosynthesis and functions of this novel genus of specialized pro-resolving mediators (SPM). SPM include 3 families of chemical mediators: resolvins, protectins and the most recent addition, maresins. These are local autacoids biosynthesized from essential omega-3 fatty acids (EPA and DHA) and possess potent multi-pronged anti-inflammatory, pro-resolving and microbial clearance actions in murine models of sepsis. The actions of SPM proved potent, cell type-specific and stereoselective with isolated human cells and in animal diseases. Endogenous formation of resolvins and protectins and their organ-protective roles were confirmed by others and extended into clinical trial. For example, SPM have potent actions in murine ischemic renal injury, obesity-induced insulin resistance and liver disease, murine colitis and arthritis, as well as reducing pain. New results indicate that resolvin regulates microRNAs in a receptor-dependent manner. Identification of endogenous SPM biosynthesized locally and temporally during acute inflammatory responses indicates that the resolution of acute inflammation is an active programmed process that also stimulates tissue regeneration. These findings change the $>200$ year old concept that resolution of inflammation is a passive process. Together, these findings indicate that natural resolution pathways may underlie many prevalent diseases associated with uncontrolled inflammation and open the potential for resolution-based pharmacology. [Authors acknowledge support of NIH grants GM095467, GM038765, DE019938 and NS067686].

\section{4}

\section{Integration of Geonomics, Proteomics, \& Metabolomics of Lipid Metabolism}

\section{Edward A. Dennis \\ Distinguished Professor of Chemistry, Biochemistry, and Pharmacology, University of California at San Diego, La Jolla, California, USA}

The omics evolution began at the end of the 20th century with the cloning of the human genome. The 21 st century has already seen the development of comprehensive proteomics analyses, but the emerging evolution is to metabolomics, the definition of which is the iden- tification and quantitation of all of the molecular constituents of the cell including its nucleic acids, amino acids, sugars, and fats. But by far, the largest number of distinct molecular species in cellular metabolism lies in the fats (or lipids) where tens of thousands of distinct molecular species exist in cells and tissues [Dennis, Proc.Natl.Acad. Sci.U.S.A., 106, 2089-2090 (2009)]. We have now applied novel liquid chromatographic-mass spectrometric based lipidomics techniques termed "CLASS" [Harkewicz \& Dennis, Annual Reviews of Biochemistry, 80, 301-25 (2011)] generally in the context of an overall omics analysis of immunologically-activated macrophages integrating transcriptomics, proteomics, and metabolomics of lipid metabolites [Dennis et al, J Biol Chem, 285, 39976-85 (2010)]. As part of the LIPID MAPS Consortium [see, LIPID MAPS/Nature Lipidomics Gateway www.lipidmaps.org], our laboratory has developed a robust and comprehensive approach to the lipidomic analysis of hundreds of fatty acids, acylethanolamines and inflammatory eicosanoids, including their numerous metabolites arising from an array of cyclooxygenases, lipoxygenases, cytochrome P450s and nonenzymatic oxidation producing isoprostanes, as well as combinations thereof [J. Lipid Res. 50, 1015-1038 (2009)]. We will discuss the application of lipidomic analysis to characterize cellular lipid signaling of Toll-like (TLR) and purinergic receptors and their "synergy" in endotoxin stimulated macrophages as models for inflammation and infection [J. Biol. Chem., 282, 22834-22847 (2007)]. New results comparing various primary macrophages and analysis of the fluxes of metabolites as well as "directed proteomics" of the systtem will be presented. Also lipidomic analysis of cells supplemented with small amounts of the omega-3 fatty acids eicosapentaenoic acid (EPA) or docosahexaenoic acid (DHA) provides information on the overall effects of EPA and DHA on the inflammatory eicosadome. Human plasma has also been profiled to quantify almost six hundred distinct lipid molecular species present across all mammalian lipid categories [J Lipid Res., 51, 3299-3305 (2010)] and the implications for the future of clinical medicine and the understanding of the mechanisms of disease will be discussed [Quhenberger \& Dennis, New England Journal of Medicine, 365, 1812 (2011)]. [Supported by LIPID MAPS Glue Grant U54 GM069338, R01 GM020501, and R01 GM064611]. 


\section{Epigenetics in Human Development and Chronic Diseases}

\section{5}

Nutritionally Programmed Influences on Gene Expression-a Mechanism Underlying the Developmental Origins of Health and Disease

\section{Susan Ozanne}

Department of Clinical Biochemistry, Institute of Metabolic Science, University of Cambridge, Cambridge CB2 2QR, UK

It is well established that there is a relationship between patterns of early growth and subsequent risk of diseases such as type 2 diabetes, cardiovascular disease and the metabolic syndrome. Studies of identical twins, individuals who were in utero during periods of famine, randomized intervention studies and animal models have provided strong evidence that the early environment, and in particular early nutrition, plays an important role in mediating these relationships. These observations underlie the developmental origins of health and disease hypothesis. The mechanisms mediating such developmental programming are still poorly defined. A number of potential mechanisms have been suggested including: (1) Permanent structural changes in an organ, such as the endocrine pancreas, resulting from suboptimal levels of an important factor during a critical period of development. (2) Permanent effects on regulation of cellular ageing through increases in oxidative stress leading to macromolecular damage, including that to DNA and (3) Epigenetic mechanisms (e.g. changes in DNA methylation, histone modifications and expression of miRNAs) leading to changes in gene expression. These epigenetic mechanisms could operate at either the transcriptional or post-transcriptional level. Several transcription factors have been shown to be susceptible to programmed changes in expression through changes in DNA methylation and histone modifications. More recently, miRNAs have been shown to be regulated by maternal diet. Transcription factors and miRNAs are both conceptually attractive targets of programmed epigenetic regulation, as through regulation of their expression a whole network of other genes will be regulated. Further understanding of the extent and nature of such processes will enable the development of preventative and intervention strategies to combat the burden of common diseases such as type 2 diabetes and cardiovascular disease.

\section{6 \\ The Epigenome as a Promising Target for Breast Cancer Prevention with Bioactive Food Compounds}

Thomas Ong

\section{Faculty of Pharmaceutical Sciences, Food and Nutrition Research Center, University of São Paulo, Brazil}

Epigenetic deregulation manifests throughout the carcinogenic process and have been implicated in breast cancer development. Global DNA hypomethylation, gene promoter hypermethylation and histone onco-modifications are associated with genomic instability and aberrant expression profiles of genes involved with cell proliferation, death and differentiation in breast cancer cells. Bioactive food compounds with anticancer potential include polyphenols from tea, soy and curcuma, sulfur-containing compounds from cruciferous vegetables, selenium, folic acid, retinoids and fatty acids. Accumulating evidence shows that these dietary bioactive compounds modulate epigenetic processes by interefering with different components of the epigenetic machinery. Because epigenetic marks are potentially reversible and early altered during carcinogenesis, the epigenome represents a promising target for breast cancer prevention with bioactive food compounds. Furthermore, because diet-epigenome interactions are likely to occur in utero, the impact of earlylife nutrition on breast cancer risk programming should be further investigated.

017

\section{Epigenetics as a Mediator of the Impact of Nutrition on Bowel Cancer Development}

John C. Mathers

Human Nutrition Research Centre, Institute for Ageing and Health, Newcastle University, Newcastle on Tyne, NE4 5PL, UK

There is compelling evidence that lifestyle factors, especially diet, are major modulators of bowel cancer risk. However, although there is evidence for cancer-related phenotypic changes in response to some dietary interventions ${ }^{1}$, the molecular mechanisms through which dietary factors modulate the acquisition or repair of the genomic damage which is fundamental for tumorigenesis remains poorly understood. We have hypothesised that poor dietary choices and/or increased adiposity may enhance bowel cancer risk through increased inflammation in the colorectal epithelium. This inflammatory load, together with other stressors e.g. altered redox state, may be responsible for aberrant gene expression though epigenetic mechanisms. Epigenetics describes the information overlaid on the primary genetic sequence which is used to regulate gene expression under specific circumstances. Epigenetic marks and molecules are plastic in response to a wide range of environmental exposures, including dietary factors ${ }^{2}$ and provide a clear mechanistic link with the aberrant gene expression which is a cardinal feature of tumours. Using both proteomic and sensitive gene methylation approaches, we have 
demonstrated that the macroscopically normal mucosa of those at higher bowel cancer risk can be distinguished from that of normal (low risk) individuals. ${ }^{3,4}$ The genes showing differential patterns of methylation in those at greater bowel cancer risk included genes in the WNT signalling pathway e.g. APC, SFRP4 and WIF1 and other tumour suppressor i.e. HPP1, P16 and ESR1.4 Such observations suggest that there may be field changes in the vulnerable large bowel epithelium which are i) detectable long in advance of any evidence of colorectal mucosal pathology and ii) potential biomarkers of risk which could be used as surrogate endpoints in dietary intervention studies. We have undertaken a randomised controlled trial (the DISC Study) in which 75 healthy volunteers were given supplements of resistant starch (RS) and polydextrose (PD) for 50 days in a $2 * 2$ factorial design. We have collected mucosal biopsies before and after the intervention to identify genes which are potential diet-responsive epigenetic markers and which could be used as surrogate endpoints for nutritional intervention studies. The DISC Study is funded by the Biotechnology and Biological Sciences Research Council (BBSRC) (Grant no. BH090948).

1. Dronamraju SS et al. (2008) Gut 58, 413-420. Cell kinetics and gene expression changes in colorectal cancer patients given resistant starch - A randomised controlled trial.

2. Mathers JC et al. (2010) Adv Genet 71, 3-39. Induction of epigenetic alterations by dietary and other environmental factors.

3. Polley AC et al. (2006) Cancer Res 66, 6553-6562. Proteomic analysis reveals field-wide changes in protein expression in the morphologically normal mucosa of patients with colorectal neoplasia.

4. Belshaw NJ et al. (2008) Br J Cancer 99, 136-142. Profiling $\mathrm{CpG}$ island field methylation in both morphologically normal and neoplastic human colonic mucosa.

\section{8 \\ Diet and MicroRNA Regulation of Inflammatory Bowel Disease}

\section{Lynnette R. Ferguson}

Discipline of Nutrition, Faculty of Medical \& Health Sciences, The University of Auckland, Auckland, New Zealand

A range of small, single-stranded, non-coding RNA molecules have been recently found to act as negative gene-regulators in both ulcerative colitis and Crohn's disease. Various microRNAs are differentially expressed in active vs. inactive ulcerative colitis, while the microRNA profile differs betweeen ileal and colonic forms of Crohn's disease. There has been some success in identifying such differences in patient's blood samples, making microRNAs potential diagnostic biomarkers. An increasing list of foods and food components has been found to be able to both up and down regulate various types of microRNAs. These include food components such as curcumin, epigalocatechin-3-gallate, and genistein, or high fat or ethanol consumption. It is of interest that the three food components detailed here have all shown some promise, at least in animal studies, in controlling symptoms of Inflammatory bowel diseases, and this may be through their effects on microRNAs.

\section{Gene-Nutrient Interaction in Metabolic Control}

\section{9
Regulation of Lipogenic Gene Expression Profile and Adiposity by the Nutrient Sensors mTOR e PPAR $\gamma$

William Festuccia

Institute of Biomedical Sciences, University of São Paulo, Brazil

Overnutrition plays an important role in the development of obesity, a highly incident disease defined as an excessive body lipid accumulation. In obesity, body tissues adjust their metabolism to coupe with a chronic exposure to an excess of nutrients. In this sense, the nutrient sensors receptor peroxisome proliferator activated receptor (PPAR) $\gamma$ and mammalian target of rapamycin (mTOR) seem to play a major role translating this nutrient excess in higher adiposity. Activation of the PPAR $\gamma$, a lipid sensor that regulates adipogenesis and adipocyte lipid metabolism, is associated with marked fat accretion in subcutaneous white adipose and brown adipose tissues. Such fat accretion is mainly attributable to the vastly enhanced uptake and storage of circulating lipids due to increased expression of genes involved in lipid uptake and esterification. Along with PPAR $\gamma$, expansion of fat mass in obesity is also associated with marked activation of the amino acid sensor mTOR in adipose tissue. Accordingly, chronic pharmacological or genetic inhibition of the mTOR signaling pathway is associated with a reduction in adipose tissue mass due to both reduced adipocyte size and number. Considering that PPAR $\gamma$ and mTOR activation are associated with fat accretion in vivo we carried out several experiments to investigate the relationships between these two nutrient sensors in the regulation of adipose tissue gene expression profile, lipid storage and metabolism. From these experiments, we have gathered strong evidences of a model of positive feed-forward regulation between the nutrient sensors PPAR $\gamma$ and mTOR, where PPAR $\gamma$ activation stimulates adipose tissue mTOR activity that, in turn, further enhances PPAR $\gamma$ transcriptional action towards some specific genes. Thus, in situations of nutrient abundance such as obesity, mTOR activation would enhance adipose tissue PPAR $\gamma$ transcriptional activity, thereby enhancing the ability and propensity of some adipose depots to store the excess nutrients as triacylglycerol. 
020

\section{Energy-Sensing Changes Induced by Perinatal Nutrition and their Implications for the Development of Metabolic Disorders}

Francisco Bolaños-Jiménez*, Omar Guzmán-Quevedo, Raquel da Silva Aragão, Georgina Pérez García, Raul Manháes de Castro

Université de Nantes, Nantes, France

A large body of epidemiological and experimental studies has demonstrated that pre- and postnatal nutrition determines the risk of developing metabolic diseases later in life. Actually, perinatal nutrient-restriction, maternal obesity or neonatal over-feeding all result in hypertension, obesity and insulin resistance in adulthood. These observations have led to the concept of developmental programming or the developmental origins of health and disease (DOHaD). According to this hypothesis, an imbalanced nutritional environment during in utero and/or neonatal development sensitises the offspring to the development of obesity and metabolic diseases via epigenetic changes that fix the effects of early, and transient, nutritional events leading to long-term changes in feeding behaviour and energy homeostasis. The fact that diverse nutritional interventions in pregnancy establish common phenotypic outcomes in the resulting offspring, suggests that sub-optimal or excessive nutrition during early development affect a common set of genes or signaling pathways that might be similar across diverse cell types. At the present time these genes and pathways are unknown, but might reasonably be proposed to be transcription factors, genes involved in regulation of the cell cycle, cell/tissue differentiation or nutrient sensing. To get insight into the shared cellular responses that may ultimately constitute a common nutritional programming mechanism, we have examined the consequences of perinatal protein restriction on the phosphorylation (activity) levels of the protein kinases mTOR and AMP, which are critical regulators of nutrient sensing and cellular metabolism. Under ad libitum feeding conditions, the energy-sensing activity of mTOR and AMPK matched the nutritional status of the animals. That is, the phosphorylation of mTOR was decreased while that of AMPK was enhanced in undernourished rats. These effects were observed in skeletal muscle but not in liver. Fasting induced a decrease in the phosphorylation levels of mTOR in liver and skeletal muscle of control but not of undernourished animals. In contrast, there were no differences in the activity of AMPK in response to fasting between the two groups. In response to fasting, the activity of mTOR within the arcuate nucleus (ARC) of the hypothalamus decreased by $30 \%$ in control as well as in undernourished rats. However, after a re-feeding period of $3 \mathrm{~h}$, the phosphorylation levels of mTOR in the ARC of control, but not of malnourished rats, were identical to those found in ad libitumfed animals. Moreover, in contrast to control animals, undernourished animals exhibited a significant reduction in the activity of mTOR in the paraventricular nucleus (PVN) in response to fasting. Altogether, these observations suggest that central and peripheral adaptation of nutrient sensing to an inadequate foetal and neonatal energetic environment is one of the basic mechanisms of the nutritional programming of metabolic disorders.

\section{1}

\section{Hypothalamic Dysfunction in Obesity}

Licio A. Velloso

University of Campinas, Brazil

The hypothalamus plays a central role in the control of food intake and energy expenditure. This activity depends on a number of environmental and genetic cues. Recent studies have identified mechanisms linking the exposition to western diets to activation of inflammation in the hypothalamus. Upon diet-induced inflammation, hypothalamic neurons become less responsive to hormonal and nutrient signals that control feeding and thermogenesis. Saturated fatty acids activate microglia leading to the local production of inflammatory factors such as TNF-a and IL1b. Once exposed to this inflammatory environment, neurons become resistant to anorexigenic/ thermogenic factors such as leptin. One of the outcomes of this inflammatory activity is the induction of neuronal apoptosis. In this presentation we will discuss the main mechanisms leading to hypothalamic dysfunction in obesity. The main nutrients responsible for this effect will be introduced and nutritional alternatives for correcting the phenotype will be presented.

\section{2 \\ Control of the Kidney Inflammation by Short Chain Fatty Acid}

Niels Olsen Saraiva Câmara

Institute of Biomedical Science, University of Sao Paulo, Sao Paulo, Brazil

Acute kidney injury is a frequent event in hospitalized patients. Its occurrence is associated with high mortality rates, and when it occurs in septic patients augments, the mortality rates up to $70 \%$. Kidney inflammation is one major mechanism in the acute kidney injury physiopathology that contributes for damage tissue. This injury is characterized by increased expression of cytokines and chemokines and activation/recruitment of leucocytes cells, as neutrophils macrophages and dendritic cells. Short chain fatty acids (SCFA) acetate, butyrate and propionate, which are produced through carbohydrate fermentation from intestinal microbiota, seem to have anti-inflammatory roles, although their role in acute kidney injury remains unveiled. By working with a renal ischemia and reperfusion model, we were able to demonstrate that the treatment with all SCFAs ameliorates renal function after kidney injury, being the acetate treatment showed the best protection, considering serum creatinine and urea levels. This better function after acetate treatment was associated with lower levels of mRNA in kidney tissue of the chemokines and pro-inflammatory cytokines along with a lower protein level of these molecules. In addition, we observed an inhibition of NFkb signaling. It was also observed that the treatment of acetate reduced the frequency of infiltrating activated dendritic cells, macrophages and neutrophils as well the apoptosis of tubular epithelial cells in kidney tissue. In conclusion, the SCFA treatment protects mice from the AKI through decreasing inflammatory process, being a promising tool to management inflammatory process in kidney. Support: Fapesp and CNPq. 


\section{Gene-Nutrient Interaction in Obesity and Diabetes}

\section{3 \\ Gene-Nutrient Interaction in Obesity and Diabetes}

Hannelore Daniel

Molecular Nutrition Unit, Technische Universität München, Gregor-Mendel-Strasse 2

D-85350 Freising-Weihenstephan, Germany

Human metabolism is the expression of a genome-environment interaction with the most complex interrelationships. The individual's genome is exposed life-long to literally hundreds of nutrients and non-nutrient compounds with highly variable concentrations. Dietdependent transcription factors and diet-dependent changes in hormone levels alter gene expression, protein turnover and the protein functions in the most complex way in an organ-dependent, cell-specific and timed manner on the background of the individual's "genetic make-up". However, with genetics, transcriptomics, proteomics and metabolomics technologies we are now able to assess the effects of diet at all levels of biology, from genome to phenotype. Although omics applications in humans are often limited by the availability of biosamples, DNA is easily obtained and is used for SNP-Analysis or whole genome sequencing. Despite enormous efforts, genome-wide association studies (GWAS) for assessing genetic determinants of diseases have so far only shown very weak effects of heterogeneity in individual genes in association type 2 diabetes (NIDDM) development. Some 40 different susceptibility genes have been identified from which the TZF7L2 gene in all ethnic groups represents the most relevant individual gene with the risk allele providing an increase in risk of around $35 \%$ whereas most of the other genes contribute much less. Although additive effects of all risk alleles predict well the fasting blood glucose level in an individual, genotyping improves the quality of diagnosis of insulin resistance (IR) or NIDDM by only $2 \%$. Analysis of changes in the transcriptome, proteome or metabolome in tissues such as muscle or adipose tissue revealed the difficulties to obtain robust and coherent data sets that could serve as biomarkers or provide novel insights into impaired organ functions in IR or NIDDM. The most convincing data are from metabolomics approaches that consistently identified (in plasma and urine) a subset of metabolites with significant associations to NIDDM with a proper predictive power. In summary, nutrigenomics is far from delivering predictive parameters for personalized health and/or disease prevention. What we needed are well-controlled long-term trials with robust clinical endpoints that allow time-dependent trajectories of all biological parameters to be recorded in the context of the individual moving from a health to disease state.

\section{4 \\ Classical and Novel Gene Targets of Long- Chain Omega3 Fatty Acids and their Potential Role in the Prevention of Obesity- Linked Diabetes}

Phillip J. White, Jing X. Kang, André Marette

Laval University, Quebec, Canada

Dietary administration of $\omega-3$ polyunsaturated fatty acids (PUFA) is often associated with altered adipose tissue (AT) morphology/function in obese mice. However, it is unclear whether these observations are an indirect consequence of reduced weight gain or result from direct actions of $\omega-3$ PUFA. Here we studied the AT of fat- 1 transgenic mice that convert endogenous $\omega-6$ to $\omega-3$ PUFA. These mice display equivalent weight gain and fat accretion to their wild-type (WT) counterparts. We performed Affymetrix microarray in epididymal AT of high fat-fed fat-1 mice and their WT counterparts. GenMAPP/MAPPFinder 2.0 pathway analysis highlighted adipogenesis, cholesterol biosynthesis, insulin signaling, prostaglandin synthesis/regulation and small ligand GPCRs as key areas where differentially expressed genes were significantly overrepresented. In accordance with the pro-adipogenic expression profile, histological analysis revealed a left shift in adipocyte size distribution in transgenic animals. Furthermore, real-time RT-PCR confirmed that mRNA for PPAR $\gamma$ was upregulated in fat- 1 mice. Transgenic restoration of $\omega$-3 PUFA also enhanced endocannabinoid receptor expression while downregulating expression of the endocannabinoid catabolic enzyme Faah. These data reveal that $\omega-3$ PUFA coordinately regulate AT gene expression programs in a manner that is independent of restriction of weight gain or fat accrual and highlight an important influence of $\omega-3$ PUFA on adipogenesis and endocannabinoid pathways in AT.

\section{5 \\ Dietary Interventions in Obese People as Affected by the Genotype: Nutrigenetic Approaches}

\section{J. Alfredo Martinez \\ Institute of Nutrition and Food Sciences, University of Navarra, 31008 Pamplona Spain}

The excessive fat accumulation occurring in obesity is the result of a disequilibrium between energy intake and expenditure, which partly depends upon genetically regulated processes affecting appetite, adipogenesis, thermogenesis, etc.. There are several plausible scenarios for the interaction between genetic and environmental factors (Diet, exercise ...) on obesity incidence, since individuals inherit a number of gene variants in key loci, but they also make specific lifestyle choices concerning dietary and activity patterns that affect weight maintenance. Gene-Nutrient interactions arise when the response of a phenotype (e.g., fat mass changes) to external impacts (e.g., overfeeding) depends on the individual's genetic background. The gene-environment relationship is a key issue in designing appropriate treatments such as 'personalised nutrition'. Thus, the large- 
scale intervention trials NUGENOB or DIOGENES and other projects investigated the interaction of more than 40 genetic polymorphisms on weight loss with low-calorie diets, which evidenced not only that some genes could control weight loss outcomes, but that much work is required in this area since gene expression profiling and epigenetics processes may be also involved Clinically relevant data have been reported about the involvement of different SNPs concerning genes regulating food intake (MC4R, LEP, FTO, etc), fat metabolism and thermogenesis, (PPARG, ADBRs, UCP's, etc), inflammation and signaling (IL-6, ADIPOQ, etc), which produced different genetically-mediated responses to energy-restricted diets or macronutrient (fat, fiber, etc) content of the dietary treatment concerning weight lowering, insulin sensitivity, lipid markers, satiety, etc. Summing up, this contribution will show that different dietary strategies based on energy restriction or macronutrient distribution manipulation devised to induce weight/fat loss were differentially effective depending on the genotype of the treated obese, whose nutrigenomically characterization will contribute to a more precise diagnosis and personalized based treatments.

1. Marti A, Goyenechea E, Martínez JA. Nutrigenetics: a tool to provide personalized nutritional therapy to the obese. J Nutrigenet Nutrigenomics 2010;3(4-6):157-169.

2. Steemburgo T, Azevedo MJ, Martínez JA. [Gene-nutrient interaction and its association with obesity and diabetes mellitus]. Arq Bras Endocrinol Metabol 2009;53(5):497-508.

3. Martinez JA, Parra MD, Santos JL, Moreno-Aliaga MJ, Marti A, Martinez-Gonzalez MA. Genotype-dependent response to energyrestricted diets in obese subjects: towards personalized nutrition. Asia Pac J Clin Nutr 2008;17(Suppl 1):119-122.

\section{6}

\section{Genetic Determinants of Obesity and Weight Changes in Response to Obesity Treatments}

\section{Louis Pérusse \\ Department of Kinesiology, Laval University, Quebec, Canada}

Reduced physical activity and increased food intake are considered as the two principal factors responsible for the dramatic increases observed in the prevalence obesity worldwide. Although this "obesogenic" environment has contributed to the shift of the distribution of BMI at the population level, not everyone in the population is overweight or obese. This suggests that genetic factors are responsible for explaining individual differences in the susceptibility (or resistance) to obesity. This gene-environment interaction is not only important in determining and individual's susceptibility to become obese, but can also influence changes of body weight in response to diet and/or exercise. The objective of this presentation is to review the evidence for gene-environment interactions in the regulation of body weight with emphasis on gene-nutrient interactions in the context of obesity. Overfeeding studies in monozygotic twins have clearly shown that the response to a caloric surplus or a caloric deficit is strongly influenced by the genotype of the individual. Changes in body weight in response to lifestyle interventions are partly heritable and several candidate gene polymorphisms have been associated with the response of obesity-related phenotypes to obesity treatments such as diet, exercise or surgery. A brief overview of the candidate gene polymorphisms that have been shown to influence body weight changes in response to various obesity treatments will be given. There is now increasing evidence that susceptibility to obesity and outcome of weight loss interventions are genotype dependent and that multiple genes are probably involved. Research in nutrigenomics and nutrigenetics have the potential help identify subjects at risk of obesity as well as those who might profit the most from various interventions to treat obesity.

\section{Gene-Nutrient Interaction in Diet and Cancer}

\section{7 \\ Omega-6/Omega-3 PUFA Ratio and Tumorigenesis}

Jing $X$. Kang

Laboratory for Lipid Medicine and Technology, Department of Medicine, Massachusetts General Hospital and Harvard Medical School, Boston, Massachusetts 02114, USA

The imbalance of omega- 6 to omega- 3 fatty acids in the human diet and body is now recognized as a modern nutritional problem important to human health. Whether or not the omega-6/omega-3 fatty acid ratio plays a critical role in cancer development has recently received attention and is under intensive investigation. Omega- 6 and omega- 3 fatty acids are essential nutrients that cannot be synthesized de novo by the human body and are mainly acquired through dietary means. These two classes of fatty acids compete with each other for the same metabolizing enzymes, but generate functionally distinct metabolites. Tumor initiation and progression require a tumor-supporting microenviroment, especially inflammation and angiogenesis. Omega- 6 and omega- 3 fatty acids differentially modulate the production of key factors or mediators that regulate inflammation, angiogenesis and proliferation, and thereby influence tumorigenesis differently. We have used the novel transgenic fat- 1 mouse as a genetic model of a balanced omega-6/omega-3 fatty acid ratio to examine its impact on tumorigenesis in different types of cancer. The data derived from the fat-1 mouse studies demonstrate that an increased tissue status of omega-3 fatty acids and a balanced omega-6/omega-3 ratio may reduce tumor formation and progression. The results also suggest that modulation of inflammatory state by omega-3 and omega- 6 fatty acids might be a key mechanism underlying their effects on tumorigenesis. Our findings implicate that a high tissue omega-6/omega-3 fatty acid ratio may be a risk factor for cancer, and that balancing the omega-6/omega-3 ratio may be an effective and safe strategy for cancer prevention and treatment. 


\section{Translation of Nutrigenetic/ Nutrigenomic Knowledge}

\section{8 \\ Nutrigenomics Research: Personalized Nutrition and Medicine}

Jim Kaput

Head. Clinical Translation Unit. Nestlé Institute of Health Sciences. Lausanne, Switzerland. For the Delta Vitamin

Obesity Research Team

Current human nutritional and genetic epidemiological methods yield "risk factors" called population attributable risks (PAR). These risk factors are statistical estimates of the percentage reduction in disease in the population if the risk were to avoided or the gene variant were not present - these measures are often assumed to apply to individuals who are likely to differ in genetic make-up, lifestyle, and dietary patterns than those individuals in the study population. Developing individual risk factors in light of the genetic diversity of human populations, the complexity of foods, culture and lifestyle, and the variety of metabolic processes that lead to health or disease are significant challenges for personalizing dietary advice for healthy or medical treatments for individuals with chronic disease. We have developed novel research strategy and analytical process that associates patterns of metabolites to genome wide genetic variations. Data for this approach was obtained in a community based participatory research paradigm that conducts basic research and community health programs.

\section{9}

\section{Caenorhabditis Elegans as a Predictive Model for a Nutrigenomics Approach to Identify New Mechanistic Insights on Potential Probiotic Bacteria}

\begin{tabular}{|c|}
\hline 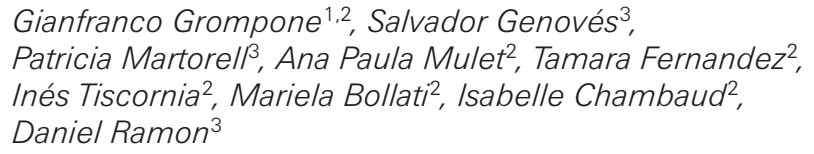 \\
\hline $\begin{array}{l}\text { anone Research, Gut \& Microbiology Platform, RD128. } \\
\text { 'enue de la Vauve, } 91767 \text { Palaiseau, France; }{ }^{2} \text { Institut } \\
\text { steur de Montevideo, Valorization Unit, Mataojo 2020, } \\
\text { 11400, Montevideo, Uruguay; }{ }^{3} \text { Biopolis S.L., Parc } \\
\text { entífic Universitat de Valencia-Edificio 2, C/Catedrático } \\
\text { E. Benlloch, no 9,46980 Valencia, España }\end{array}$ \\
\hline
\end{tabular}

The human immune system must deal with the paradox of destroying dangerous bacterial pathogens while preserving the beneficial gut microbiota which colonizes the intestine in huge numbers. Three key players mediate immune tolerance: the microbes themselves, the gut epithelium and the immune cells, especially within the lymphoid tissue of the lamina propia. Getting new insights on how gut homeostasis is established, maintained or disrupted is crucial to better understand the role of the gut commensal microbiota in the health status of the host. There is increasing evidence that probiotics play specific roles as modulators of inflammatory pathways by reducing tissue inflammation or having antioxidant effects. We develop here a new functional screening method using the nematode Caenorhabditis elegans as a host to identify and characterize new potential antioxidant probiotic strains. We fed C. elegans with 77 Lactic Acid Bacteria (LAB) strains, and viability of worms upon an $\mathrm{H} 2 \mathrm{O} 2$ oxidative stress treatment was assessed. Lifespan of worms fed with selected probiotic candidates was also studied. We obtained 3 potential probiotic strains in C. elegans. This approach was complementary to a multidimensional integrative screening for anti-inflammatory effects in vitro. We showed positive correlations of the selected strains between anti-inflammatory in vitro profiles and oxidative stress resistance in C. elegans. Moreover, we developed a nutrigenomic approach where 3 days-old (young) and 10 days-old (adult) C. elegans were fed with selected probiotic candidates. Transcriptomic analysis of worms fed with these strains showed modulation of highly conserved cell signaling pathways from nematodes to mammals. Future work will focus on mechanistic studies in higher animal models. (Funding: ANII: PE_ALI_1_1702, Danone Research).

\section{0 \\ Practical Application of Nutrigenomics for the Development of an Oral Cosmetic Anti- Wrinkle Supplement}

\author{
Robin van den Berg*, Linda J. Wainwright*, \\ Karen E. Barrett*, John Casey*, Gail Jenkins* \\ \#Unilever R\&D, Vlaardingen, PO Box 114, 3130 AC \\ Vlaardingen, The Netherlands; *Unilever R\&D, Colworth \\ Science Park, Sharnbrook, Bedfordshire MK44 1LQ. UK
}

Application of nutrigenomics to skin health presents some unique options. It is well established that the skin tissue can obtain benefits from specific nutrients given orally. Studies that have performed previously provided evidence for the interaction of nutrients with the genome demonstrating marked changes in gene expression and the ability of genetic variations to cause marked differences in individual response to dietary factors. Our in vitro data already indicated that several genes encoding proteins vital for good skin tone, such as decorin and several anti-inflammatory molecules, could be upregulated with dietary ingredients. We therefore conducted a double-blind, placebo-controlled human intervention study for 14 weeks to evaluate the effect of a soya based drink on skin ageing parameters. The main actives that were used were isoflavones from soya which activate oestrogen receptors that trigger the skin to make collagen and omega-3 polyunsaturated fatty acids from fish oils which can activate PPAR which is involved in collagen synthesis. Also vitamins C, E and lycopene were included to promote general tissue health. Healthy, non-smoking Caucasian women (skin type II-III), aged 45-65, were recruited. At the end of the intervention, a statistically significant reduction in wrinkle depth $(R z)$ in the test group compared to placebo 
was found. Moreover the treatment had the greatest effect on deepest wrinkles. In addition, skin biopsies were analysed for pro-collagen I synthesis (new collagen) and a significant increase in pro-collagen I synthesis in biopsies from the test group at the end of the study when compared to the placebo was found, with an average increase in procollagen levels of $25 \%$. This human study has demonstrated that skin anti-ageing effects can be obtained through consumption of a soy isoflavone-rich drink. In particular, we were able to demonstrate a reduction in wrinkle depth in the crow's foot area of the eye and that this change appears to be underpinned by an increase in new collagen synthesis over a 14 week period.

\section{1}

\section{From Micro-Array to Daily Meal Tray}

\section{Jean-Michel Antoine}

Danone Research, France

An adequate nutrition is beneficial for Health, and Recommended Dietary Allowances and Food Dietary Guidelines are providing average targets for the general population. On the other hand the general population has developed a diversity of physiological pathways to be able to cope with the large variety of food on Earth, and to increase its survival capacity. Nutrigenomic is exploring how genes control nutrition, and how nutrition modulates genes. Nutrigenomic adds complexity to Nutrition science, provides new tools to adapt food to specific nutritional needs, and new markers to demonstrate the benefits of nutrients, foods and diets. Genes are sensitive to nutrition as demonstrated by epigenetic. Many genomic pathways are sensitive to different nutrients either before, during or after transcription of DNA into proteins. Most of "our" genes are within our microbial compartments, and they are easily changed: either turned on or off, or coming in/out with new micro-organisms or new concentrations of existing micro-organisms, or new levels of expression.

One specific area we are interested in is gut genomics:

- Gut microbiota can modulate gut genes expression and modulate food efficiency from Energy to bio-actives.

- Food can modulate Gut microbiota composition and functions with Prebiotics.

- Probiotics can modulate Gut microbiota composition and functions and modulate gut genes expression.

Finally Nutrigenomic is a perfect example for a timely ethical debate: When scattered science should be translated into practical applications and communicated to consumers. We should keep in mind that the discovery of the beneficial effect of lime on scurvy (Lind 1742) was used by Cook many years before the official recognition the benefits of lime. It is the down side of precautionary principle. On the other hand, costs of demonstration should not add another barrier to offering adapted products to those who need them most.

\section{Gene-Nutrient Interaction in Exercise, Chronic Diseases and Skeletal Muscle Function}

\author{
032 \\ Molecular Mechanisms Involved in Skeletal \\ Muscle Atrophy
}

Anselmo Moriscot

Institute of Biomedical Sciences, University of São Paulo, Brazil

Skeletal muscle atrophy is marked by protein loss, leading to functional deficit and decreased quality of life. Skeletal muscle atrophy is triggered by several circumstances including mechanical unloading such as immobilization and space flights and metabolic conditions such as sepsis, cancer, renal failure, AIDS. Although it is well known that skeletal muscle atrophy occurs due decreased protein synthesis and increased protein degradation, the underlying mechanisms are still under investigation. In this talk we will first review the current knowledge upon structural, cellular and molecular aspects of skeletal muscle atrophy, also making correlations with hypertrophy and regeneration. We will then focus on results from our group regarding the impact of leucine in skeletal muscle atrophy (immobilization model) at specific fiber types, and the effects of leucine supplementation in vivo upon protein synthesis and degradation. The impact of HMB, a leucine metabolite, in atrophying muscles will also be addressed. Emphasis will be given to the proteasome system, a key proteolitic system in skeletal muscle. In addition to the immobilization model, we will also present results on the impact a hormonal model (thyroid hormone), upon the proteasome system. We are also going to present results on the role of specific E3 ligases (key enzymes in the proteasome system) upon skeletal muscle fiber mass.

033

\section{Catalase Overexpression and Mitochondrial Function in Insulin Resistant Muscle Cells}

Leonardo R. Silveira, Marina R. Barbosa, Igor H. Sampaio, Luciane C. Alberici, Isis C. Kettelhut, Claudio Zoppi, Everardo M. Carneiro, Rui Curi

School of Physical Education and Sports of Ribeirao Preto, Department of Biochemistry and Immunology, University of Sao Paulo, Ribeirao Preto, ICB - USP, São Paulo, IB-Unicamp, Campinas, Brazil

We examined whether the reduction of mitochondrial capacity and insulin response imposed by the high availability of fatty acids are associated with an increase of intracellular hydrogen peroxide $\left(\mathrm{H}_{2} \mathrm{O}_{2}\right)$. We investigated whether the oxidative stress induced by excess fatty acids can be reversed with improved antioxidant capacity 
through overexpression of catalase. Alcance um público internacional. vídeos do YouTubeThe catalase gene was successfully transfected in skeletal muscle cells through pcDNA3 plamid as indicated by catalase mRNA and content $(\mathrm{p}<0.05)$. The $\mathrm{H}_{2} \mathrm{O}_{2}$ production was increased after palmitic acid treatment. This effect markedly reduced the citrate sintase activity as well as the mRNA levels of PGC1 $\alpha(\mathrm{p}<$ 0.05 ). This effect was accompanied by a significantly reduction of $\mathrm{p}$-AKt which further was demonstrated to down regulate $\mathrm{p}$-CREB and PPAR- $\beta$. In addition, oxygen consumption and uptake glucose were markedly reduced in the presence of palmitic acid $(\mathrm{p}<0.05)$. However, the catalase transfection was observed to prevent reduction of $\mathrm{p}$-AKt level which further was demonstrated to up regulate $\mathrm{p}$-CREB and PPAR- $\beta(\mathrm{p}<0.05)$. Our results showed that fatty acid reduced the p-AKt level. This effect was associated with a low p-CREB level and consequently PGC1- $\alpha$ transcription. The reduced mitochondrial oxygen consumption favored ROS production and insulin resistance. The catalase- induced effect on p-AKt increased the oxygen consumption and glucose and fatty acid metabolism suggesting that mitochondrial biogenesis might be regulated by phosphorylated level of AKt.

\section{Oral Abstract Presentations}

\section{4}

\section{Bitter Taste Receptor Polymorphisms and Human Aging}

Daniele Campa ${ }^{*}$, Francesco De Rango ${ }^{2 *}$, Maura Carrai ${ }^{3}$, Paolina Crocco 2, Alberto Montesanto 2, Federico Canzian", Giuseppina Rose? ${ }^{2}$ Cosmeri Rizzato', Giuseppe Passarino ${ }^{2 \#}$, Roberto Barale $3 \#$

${ }^{1}$ Genomic Epidemiology Group, German Cancer Research Center (DKFZ), Heidelberg, Germany; ${ }^{2}$ Department of Cell Biology, University of Calabria, Rende, Italy; ${ }^{3}$ Department of Biology, University of Pisa, Pisa, Italy

Several studies have shown that genetic factors account for $25 \%$ of the variation in human life span. On the basis of published molecular, genetic and epidemiological data, we hypothesized that genetic polymorphisms of bitter taste receptors, which modulate food preferences, but are also expressed in a number of organs and regulate food absorption processing and metabolism, could modulate the aging process. Using a tagging approach, we investigated the possible associations between longevity and the common genetic variation at the three bitter taste receptor gene clusters on chromosomes 5, 7 and 12 in a population of 941 individuals ranging in age from 20 to 106 years from the South of Italy. We found that one polymorphism, rs978739, situated 212 bp upstream of the TAS2R16 gene, which encode a receptor for bitter molecules present in several plants, shows a statistically significant association ( $\mathrm{p}=0.001)$ with longevity. In particular, the frequency of A/A homozygotes increases gradually from $35 \%$ in subjects aged $20-70$ up to $55 \%$ in centenarians these data provide suggestive evidence on the possible correlation between human longevity and taste genetics.

\section{5 \\ Genetic Polymorphism of Homocysteine Metabolic Genes and Susceptibility of Type 2 Diabetes in Chinese}

\author{
Tao Huang, Jianqin Sun, Duo Li \\ Zhejiang University, China
}

Background: An association of genetic variants of homocysteine (Hcy) metabolic genes with type 2 diabetes (T2DM) has been reported, however, there is no data in Chinese T2DM.

Objectives: The objective of the present study was to investigate the relationship between the genetic variants in Hcy metabolism related genes and plasma Hcy levels and T2DM susceptibility in Chinese.

Methods: Seven hundred and seventy-four T2DM patients and 500 healthy subjects were recruited in China. The plasma Hcy concentration was determined, 13 polymorphisms of 6 genes involved in homocysteine/folate pathway were genotyped.

Results: The prevalence of Hyperhomocysteinemia (HHcy) in T2DM was significantly lower $(20.35 \%)$ than in healthy subjects $(26.97 \%)(p=0.023)$. MTHFR (rs1801131, rs1801133), MTR (rs1805087, rs16834521), MAT1A (rs3851059, rs4933327), PEMT (rs4646406) was significantly associated with plasma Hcy in T2DM. MTHFR (rs1801131) showed a significant association with increased risk of T2DM $(\mathrm{OR}=1.93$ for $\mathrm{CC}$ vs AA, $\mathrm{p}=0.041, \mathrm{OR}=3.13$ for $\mathrm{CC}$ vs $\mathrm{AA}+\mathrm{AC}, \mathrm{p}=0.017$, respectively). The variant MTHFD (rs2236225) also showed significant association with decreased risk of T2DM $(\mathrm{OR}=0.36$, for AA vs GG, $\mathrm{p}=0.027, \mathrm{OR}=0.36$ for $\mathrm{AA}$ vs $\mathrm{GG}+\mathrm{GA}, \mathrm{p}=0.017$, respectively). In addition, PEMT (rs4646356) variants displayed a significant association with increased risk of $\mathrm{T} 2 \mathrm{DM}(\mathrm{OR}=1.52$ for $\mathrm{CT}+\mathrm{TT}$ vs CC, $\mathrm{p}=0.042)$. However, PEMT (rs4646406) variants was significantly associated with decreased risk of T2DM (OR $=0.48$ for TT vs AA, $\mathrm{p}=0.048)$.

Conclusions: This study provides evidence that these common variants of MTHFR, MTR, MAT1A, PEMT, and MTRR are associated with alterations in plasma Hcy. MTHFR, MTHFD, and PEMT genetic variants are associated with the susceptibility to T2DM in Chinese. 


\section{Journal of \\ Nutrigenetics \\ Nutrigenomics}

\section{P001 \\ Comparative Toxicity of Fatty Acids on Human Neutrophils}

\section{Adriana Cristina Levada-Pires, Thais Martins Lima-Salgado, Tania Cristina Pithon-Curi, Raquel Zambonatto, Rui Curi \\ Universidade Cruzeiro do Sul}

In the present study the highest concentration of palmitic, stearic, oleic, linoleic, docosahexaenoic and eicosapentaenoic acids that are not toxic to human neutrophils were determined. Neutrophils from sedentary volunteers were cultivated for 3 hours with increasing concentrations of these fatty acids (from 50 to 300 microM). Cell membrane integrity, DNA fragmentation and phosphatidilserine externalization were evaluated by flow cytometry. The mechanisms involved in the toxic effects of the fatty acids (FA) on neutrophils were investigated by measuring ROS production, mitochondrial membrane depolarization, expression of bax and caspase 3, activities of caspases 3,8 and 9 and determination of intracellular neutral lipid content. The percentage of cells with intact membrane and fragmented DNA varied depending on the fatty acid. The toxic effect of the FA on human neutrophils varied irrespective of the chain length and the degree of unsaturation. The cytotoxicity was as follows: stearic $>$ DHA $=$ oleic $=$ linoleic $=$ palmitic $>$ EPA. All fatty acids induced human neutrophil death by apoptosis, however, by different mechanisms. Oleic, linoleic and DHA increased ROS production, expression of the pro-apoptotic protein bax and caspase 3, caspase 3 activity and neutral lipid accumulation. The mitochondrial membrane depolarization was induced by oleic, linoleic and palmitic acids. The saturated fatty acids did not cause the same changes, stearic acid raised caspase 3 expression and activity, whereas palmitic acid changed all parameters measured, except for neutral lipid accumulation.

\section{P002 \\ Glucagon Like Peptide 1 (GLP-1) and Central Control of Body Weight}

\section{Adriana Laura Burgueño, Noelia Luz Gonzales Mansil, Tomas Fernandez Giano, Silvia Cristina Sookoian, Carlos Jose Pirola \\ Medical Research Institute, UBA-CONICET}

Background: Glucagon Like Peptide 1 (GLP-1) is a small intestine-derived hormone secreted from gut L-cells in response to food intake. Circulating GLP-1 has a half life of 1-2 minutes owing to the dipeptidyl peptidase 4 (DPP4)-mediated-degradation. Besides its wellknown anti-hyperglycaemic effects, GLP-1 has also been involved in the regulation of food intake, body weight and cardiovascular function.
Objectives: our aim was to study whether GLP1 and leptin shared common energy balance regulation pathways.

Methods: We used 32 male 20 weeks-old Wistar rats, 8 rats were feed ad-libitum group (controls) and 24 rats were fasted for $72 \mathrm{hs.}$ During fasting, 8 rats/group, received leptin $(10 \mu \mathrm{g} / \mathrm{rat})$, exenatide $(5 \mu \mathrm{g} / \mathrm{rat})$ or vehicle (phosphate buffered saline with $0.1 \%$ bovine serum albumin) every $12 \mathrm{hs}$. We recorded daily body weight and arterial blood pressure. At the end of the experiment, animals were kill, diencephalon was dissected, plasma collected and visceral white adipose tissue weighed.

Results: Fasting produced notorious decrease in: body weight $(\mathrm{p}<$ $0.005)$, adipose tissue weight $(\mathrm{p}<0.02)$, serum glucose levels $(\mathrm{p}<$ $0.00001)$ and plasma leptin levels $(\mathrm{p}<0.0000004)$ compared with adlibitum rats. We observed that fasting produced a decrease in diencephalic expression levels of Glp-1 receptor $($ Glp1R) $(p<0.0002)$ and the long isoform of leptin receptor $(\mathrm{ObRb})(\mathrm{p}<0.05)$ versus ad-libitum group. Both, leptin and exenatide, produced similar responses. Both reduced systolic arterial blood pressure (leptin: $\mathrm{p}<0.02$ and exenatide: $\mathrm{p}<0.00005$ versus vehicle) and serum glucose levels (leptin: $\mathrm{p}<0.001$ and exenatide: $p<0.000001$ versus vehicle), increased diencephalic expression levels of pro-opiomelanocortin (Pomc) (leptin: $p<0.01$ and exenatide: $p<0.02$ versus vehicle) and have no effect on expression levels of $\mathrm{ObRb}$. On the other hand, leptin treatment increased diencephalic expression levels of Glp-1R ( $p<0.04$ vs vehicle). In addition, adipose tissue weight/body weight ratio correlated with expression levels of Glp1R $(\mathrm{R}=-0.51, \mathrm{p}<0.008)$ and diencephalic thyrotropinreleasing hormone $(\operatorname{Trh})(\mathrm{R}=-0.53, \mathrm{p}<0.04)$.

Conclusions: Our results suggest that leptin and GLP-1 produced a similar effect on some of the studied parameters. It is tempting to speculate that they would be involved in the same regulatory pathway. We postulated that the effects of Glp-1 analogues on body weight might be due to activation of the central TRH system.

\section{P003}

\section{Gene Modulation by Nutrients and Bioactive Food Compounds in the Pathogenesis of Chronic Diseases}

Ágatha C. Silva De Carvalho, Larisse Monteles Nascimento, Gabriela De Sousa Silva Rios, Ana Karolinne Da Silva Brito, Eduardo Emanuel Sátiro Vieira,

Vanessa Brito Lira De Carvalho,

Kyria Jayanne Clímaco Cruz,

Daniele Rodrigues Carvalho Caldas, Dilina do N. Marreiro

Universidade Federal Do Piauí

The completion of the human genome project has contributed to a breakthrough in research aimed at finding answers about the relationship between the pathogenesis of chronic diseases and food, more

\section{KARGER \\ (C) 2012 S. Karger AG, Basel}

Fax +41613061234

E-Mail karger@karger.ch

www.karger.com
Accessible online at: www.karger.com/jnn 
specifically about the possible interactions between genes and nutrients. Vitamin D, obtained by endogenous synthesis or by consumption of food from animal origin, binds to its nuclear receptor VDR regulating gene transcription and cellular functions in different tissues. Some studies have shown the influence of hypovitaminosis D in the etiology of cardiovascular disease, obesity, metabolic syndrome and chronic kidney disease. Resveratrol is a phytoalexin present in peanuts, blueberries, grapes and plant species. This compound has antioxidant, anti-inflammatory, anticancer and cardioprotective capabilities. The mechanisms that explain these properties refer to the power to modulate gene expression by inhibiting the activation of nuclear factor kappa B (NF-kB), Activator Protein 1 (AP-1), nitric oxide synthase (iNOS ), Jun N-terminal kinase (JNK) and 5-lipoxygenase (5-LOX), promoting an improvement in inflammatory processes and oxidative stress and suppressing tumor progression. Camellia sinensis is a plant popularly known as green tea, which has been used in diets because of its major chemical components, flavonoids and catechins, have chemoprotective, anticarcinogenic, antioxidant and anti-inflammatory actions. Among the green tea catechins, the most abundant is epigallocatechin gallate, which is an antiinflammatory and inhibits in vitro activation of NF- $\mathrm{BB}$. From these data in the literature, it is possible to identify the importance of nutrigenomics as a tool to assess the interaction between nutrients and bioactive food compounds in gene modulating and especially in contributing to the reduction of chronic diseases.

\section{P004 \\ Fish Oil and Curcumin Have No Effect on Glucose Metabolism But Improves Lipid Metabolism and Inflammation in Obesity- Induced Insulin Resistance}

\section{Agussalim Bukhari, Mardiana Mardiana, Marniar Marniar, Rohani Rohani, Radiah Radiah}

Medical Faculty, Hasanuddin University, Indonesia

Background: Obesity induced-insulin resistance has been linked to chronic low grade inflammation. On the other hand, fish oil and curcumin has been reported to have antii nflammatory and lipolytic effects. But the effects of fish oil and curcumin on preexisting insulin resistance is inconlusive.

Objectives: To examine whether fish oil and curcumin could improve preexisting insulin resistance via their anti inflammatory and fat oxidation inducing effects.

Methods: Six groups of mice were on Normal diet (ND) or High fat diet (HFD) for 12 weeks, then treated for $8 \mathrm{wk}$ as follows: 1) Normal diet, 2) HFD alone, 3) HFD with fish oil (HFD-FO), 4) HFD with curcumin (HFD-CUR), 5) HFD with fish oil and curcumin $3 \mathrm{~g} /$ $\mathrm{kg}$ diet (HFD-FO + CUR) and 6) HFD with Metformin (HFD-MET) as positive control.

Results: Glucose and Insulin tolerance test (GTT, ITT) revealed higher blood glucose levels in all groups of HFD than those of ND. Surprisingly, mice treated with HFD-FO had higher blood glucose levels than other groups during GTT and ITT. Whereas mice with HFD-FO + CUR have significantly lower blood glucose level on min 60 of GTT compared to HFD alone. Expression of TNF $\alpha$ from epi- didymal fat was significantly increased in HFD than ND and lower in groups of HFD-FO, HFD-FO + CUR and HFD-MET compared to HFD alone. On the other hand, expression of PPAR $\alpha$ in the liver and adiponectin in epididymal fat were decresead in HFD than in ND but increased in group of HFD-FO, HFD-FO + CUR and HFD-MET compared to HFD alone.

Conclusions: Fish oil does not improve preexisting obesityinduced insulin resistance, but it lowers TNF $\alpha$ and tends to increase adiponectin and PPAR $\alpha$ expression. Interestingly mice with HFD-FO + CUR have improved glucose metabolism, lower expression of TNF $\alpha$ and higher expression of PPAR $\alpha$.

\section{P005 \\ Expression of Genes Associated to Metabolism in the Heart of Infarcted Rat Supplemented with Fish Oil}

\author{
Alcione Lescano de Souza Jr., Christiane Malfitano, \\ Leandro Ezequiel de Souza, Maria Cláudia Irigoyen, \\ Rui Curi
}

ICB 1-USP

Background: Myocardium infarct size is influenced by many factors, including fatty acids. n-3 PUFAs are natural ligands of several nuclear receptors and transcription factors that regulate gene expression in the heart.

Objectives: Effects of supplementation with n-3 PUFA on expression of genes associated to metabolism in the left ventricle, infarct size and coronary blood flow were investigated.

Methods: The rats were supplemented by gavage with fish oil rich in n-3 PUFA (42 and 17\% of EPA and DHA, respectively) during 20 days before induction of infarction. The permanent ligation of the left descending coronary artery was used as model of the heart infarction. Gene expression, infarct area and blood flow were analyzed 24 hours after coronary ligation. Coronary blood flow was quantified using color microsphere technique. eNos, Glut1, HIF 1, iNOS, PFK, PDK 4 and UCP 3 genes expressions were measured by real time PCR.

Results: Fish oil group exhibited smaller infarction size (by $28 \%$ ). The infarct per se reduced by 4 times the coronary blood flow when compared with non infarcted group and fish oil supplementation prevented this reduction. In the infarcted groups, increased expression of eNos, HIF 1 and Glut 1 by fish oil supplementation was observed. There was a decrease in iNOS expression by the infarct and fish oil prevented this reduction. The infarct increased the expression of PFK, PDK4 and UCP3 genes and fish oil supplementation did not change this effect.

Conclusions: Supplementation with fish oil protected the heart from ischemic injuries and increased expression of genes associated to cell metabolism and survival.

Financial support: CAPES, CNPQ, FAPESP, NATURALIS and UNEMAT. 
P006

\section{Antiangiogenic Effect of Chilean Propolis Could Be Mediated by MicroRNAs}

Alejandro Cuevas, Nicolás Saavedra, Martina Rudnicki, Dulcineia Saes Parra Abdalla, Luis Antonio Salazar

Universidad De La Frontera

Background: Several studies have shown that polyphenolic extract from propolis can modulate angiogenesis in both in vitro and in vivo models. However, the mechanisms implicated in this effect are poorly understood.

Objectives: The aim of this study was to investigate the effect of an ethanolic-extract of Chilean propolis (EEChP) on in vitro angiogenesis and expression of angiogenic microRNAs.

Methods: In vitro "scratch wound assay", "matrigel angiogenesis assay" and the ex vivo "rat aortic ring model" were used to assess the antiangiogenic properties of the EEChP. In order to study ERK $1 / 2$ phosphorylation non stimuled human umbilical vein endothelial cells (HUVECs) were incubated in standard culture condition for 15 min and protein were extracted. HUVECs incubated under hypoxic conditions for $4 \mathrm{~h}$ (hypoxia chamber or dimethyloxallylglycine induced hypoxia) were used to evaluate the HIF1- $\alpha$ protein stabilization. Western blot was performed to assess the level of protein. HUVECs stimuled with VEGF $(20 \mathrm{ng} / \mathrm{mL})$ and FGF $(35 \mathrm{ng} / \mathrm{mL})$ were treated with EEChP $(10 \mu \mathrm{g} / \mathrm{mL})$ or vehicle for $4 \mathrm{~h}$ for total RNA (enriched with small RNAs) extraction. MicroRNAs miR-221, miR222, miR-17, miR-19b and miR-27b were studied using relative qPCR.

Results: At $10 \mu \mathrm{g} / \mathrm{mL}$ of EEChP, in vitro cell migration, formation of capillary-like structures in matrigel and the ex vivo sprouting of endothelial cells from the rat aorta fragment were attenuated. In addition, the treatment of EEChP showed no effect on HIF 1- $\alpha$ protein accumulation in hypoxia- or dimethyloxallylglycine-stimulated HUVECs, but the key step on MAPK/ERK signal pathway, ERK 1/2 phosphorylation, was inhibited. Finally, the propolis extract showed no effect on the expression level of miR-221, miR-222, miR-17 and miR-27b. However, miR-19b was overexpressed. In silico analysis suggesting that MAPK1 (ERK2) is target for miR-19b.

Conclusions: In summary, these results suggest that miR-19b and ERK 1/2 phosphorylation could be related with the antiangiogenic effect of Chilean propolis, but more studies are needed to corroborate these findings.

\section{P007 \\ Proglucagon Gene Transcriptional Level and Glucagon Like Peptide 1 Messenger in Response to Dietary Complex Carbohydrates Ingestion in Rats}

\author{
Jacira Maria Andrade Sousa, Alexandre Coelho Serquiz, \\ Fabiana M.C. Carvalho, Brenda C.O. Galvão, \\ Luciana M.A. Rabelo, Elizeu Antunes Santos, \\ Ana H.A. Morais, Thales M.C. Araújo, \\ Ana Luíza S.C. Villarim
}

Universidade Federal do Rio Grande do Norte

Background: The hypoglycemic, insulinotropic and satiety response roles of GLP-1 in to dietary carbohydrates and exercise as well as the nutrient and exercise effects on the GLP-1 production are well documented. The molecular screening to determine the role of specific dietary complex carbohydrate in triggering intestinal GLP-1 release can be explored to optimize the GLP-1 production and may be useful in planning diets for diabetic people or to prevent decreased glucose tolerance caused by other etiologies.

Objectives: To evaluate the intestinal GLP-1 transcriptional levels in response to an exercise protocol training associated with dietary complexes carbohydrates.

Methods: Wistar males rats, 21 days old were fed with hypercaloric diets and underwent protocol training during 15 days and feeding with commercial pellets, tapioca, couscous and algarroba, foods rich in complex carbohydrates. The RNA was extracted from intestines using TRIzol reagent (Invitrogen). Reverse transcription was performed with $1 \mu \mathrm{g}$ of total RNA using the first strand cDNA synthesis kit for RT-PCR (Invitrogen) with oligo $\mathrm{d}(\mathrm{T})$ as a primer. The resultant cDNA was amplified using the following primers for proglucagon gene and GLP-1 messenger evaluation (Forward Primer) $=5$ 'GTTTA CATCGTGGCTGGATTG3'(Reverse Primer) = 5 'CTATTTCTTGTCAGGATCTT3` and for G3PDH, positive control, the primers (Forward Primer) 5'TCCACCACCCTGTTGCTGTA G3 '(Reverse primer) 5'GACCACAGTCCATGACATCACT 3' were used. The RT-PCR products profile was analyzed after electrophoresis in $1.2 \%$ agarose gel in $1 \mathrm{x}$ TBE and $5 \mathrm{ug} / \mathrm{ul}$ Ethidium bromide.

Results: The blood glucose was not different between the animal groups $(\mathrm{P}>0.05)$. The body weight gain of animals in cuscuz diet showed significant differences $(\mathrm{P}<0.05)$. The RT-PCR results showed no proglucagon gene expression in rats fed with diets galactomannan rich while the transcription of this gene was increased after the ingestion of the cuscuz (Zea mays) and tapioca (Manihot sculenta).

Conclusions: Galactomannan from algarroba (Prosopis juliflora) does not have any effect on the proglucagon gene expression, using primers to amplify the gene region corresponding to the GLP-1 peptide in opposition to the ingestion of diets rich in starch from couscous (Zea mays) and tapioca (Manihot sculenta).

Financial support: UFRN/FAPERN. 


\section{P008 \\ Maternal Insulin Resistance and Exposure to High Sucrose and Fat Diet Lead to Impairment of Glucose Metabolism, Hepatic Insulin Signaling Pathway, and Global DNA Hypermethylation in the Offspring}

\author{
Alfonso Hernández, Cátia Lira Amaral, Diogo Vasconcelos, \\ Amanda Roque Martins, Marco Aurelio Vinolo, \\ William Tadeu Festuccia, Sandro M. Hirabara, Rui Curi, \\ Luis Antonio Salazar \\ U. De La Frontera, U. Sao Paulo, UC Temuco
}

Background: High sucrose and fat diets (HSFD) are known to cause liver steatosis and insulin resistance. However, maternal insulin resistance as a potential risk factor for late onset metabolic-related disorders is still unclear.

Objectives: The effect of maternal insulin resistance on glucose metabolism and insulin signaling pathway in hepatic tissue of the offspring was investigated. The involvement of DNA methylation in the changes observed was also examined.

Methods: Female Wistar rats $(10 \pm 1$ weeks $)$ were fed a HSFD $\left(\mathrm{F}_{0} \mathrm{IR}\right)$ or a balanced (control) diet $\left(\mathrm{F}_{0} \mathrm{~N}\right)$ for 8 weeks. Glucose tolerance test (GTT), plasma insulin levels, and body weight were then measured before being mated with healthy males. The male pups of F0IR (identified as $F_{1} I R$ ) and F0N (called $F_{1} N$ ), were fed from the 3 th to 8th week with standard rodent diet, and then divided in two groups each. One group of $F_{1} I R$ and one of $F_{1} N$ rats were fed a HSFD and the rest were fed with CD for 14 days. Afterwards, GTT and insulin tolerance tests were performed. After euthanasia, liver was extracted, DNA and RNA were isolated and gene expression analyses were performed using a PCR array platform for insulin signaling pathway. Global DNA methylation was analyzed with an EIA-based assay.

Results: At end of dietary manipulation, $\mathrm{F}_{0} \mathrm{IR}$ rats showed raised blood glucose and plasma insulin levels at GTT versus the F0N group, with no differences in body weight. $\mathrm{F}_{1}$ IR pups showed differences in body weight at the 3 th day but not in basal glycemia, trend that remained at the $3 \mathrm{rd}$ and 8 th weeks. After 14 days of dietary manipulation, the $\mathrm{F}_{1} \mathrm{IR}$ group fed HSFD showed impaired glucose tolerance, basal hyperinsulinemia and elevated plasma insulin levels during the GTT, in addition to increased white fat depots, liver weight and hepatic lipid content when compared with the others groups. Gene expression analyses showed overexpression of genes associated to lipid and glucose metabolism (Fasn, Pklr, Acaca, Srebfl and Pparg) and insulin-responsiveness (Tg, Prkcz, Pik3r2, Irs 1, Igfbp1, Gab1, G6pc, Akt3 and Adrald) in $\mathrm{F}_{1} \mathrm{IR}$ rats fed HSFD $(\mathrm{P}<0.05)$. Global hepatic DNA analyses showed hypermethylation in $\mathrm{F}_{1} \mathrm{IR}$ rats fed HSFD $(\mathrm{P}<0.001)$.

Conclusions: Maternal insulin resistance leads to hepatic insulin resistance, fatty liver and hepatic DNA hypermethylation.

Financial support: Conicyt-Chile, FAPESP and Guggenheim Foundation.

\section{P009 \\ Muscle-Specific MicroRNAs Are Differentially Regulated in Insulin Resistant Muscle from Diet-Induced Obese Mice}

Alice C. Rodrigues, Amanda Roque Martins,

Luis Gustavo De Sousa, Sandro M. Hirabara, Rui Curi

Institute of Biomedical Sciences, Brazil

Background: A clinically relevant question is whether microRNA (miR or miRNA) levels are capable of assessing the probability of diabetes mellitus type 2 (DM2) manifestations in patients with insulin resistance.

Objectives: The aim of this study was to investigate which microRNAs regulate the genes involved in insulin resistance in skeletal muscle after a high fat diet.

Methods: Male C57BL/6 mice received a balanced (control group) or a high-fat diet (HFD) during 4, 8 or 12 weeks (weeks 0 to 12). At the end of the treatment, soleus muscles were isolated and subjected to insulin-induced glucose uptake and $\mathrm{CO}_{2}$ production or flash-frozen in liquid nitrogen immediately for total RNA extraction. Muscle specific microRNAs (miR-1, miR-206 and miR-133a) and target mRNAs were measured by TaqMan Real-time PCR assay in soleus muscle of HFD-fed mice or control group, using snoRNA20 or Rpl (36B4), for normalization, respectively.

Results: Insulin efficiently stimulated 2-DG uptake, glycogen synthesis and $\mathrm{CO}_{2}$ production in control group and HFD-fed animals for 4, 8 and 12 weeks were unresponsive to insulin. HFD-fed animals were hyperinsulinemic after 4,8 and 12 weeks when compared to control diet-fed mice. Accordingly, Irs-1 mRNA was down-regulated after 8 weeks of HFD (HFD: $0.63 \pm 0.17$ vs control: $1.00 \pm 0.24, p=$ $0.02)$. After 8 weeks of HFD, miR-1 and miR-206 were significantly increased $(\sim 1.9$ fold, $\mathrm{p}<0.05)$ and decreased $(\sim 2$-fold $)$, respectively, when compared to control group $(1 \pm 0.66)$. MiR-133 was up regulated only after 12 weeks of feeding a HFD compared to control group $(0.66 \pm 0.22$ vs $1 \pm 0.08, \mathrm{p}<0.05)$. After, we have measured the expression of potential mRNA targets for these microRNAs, such as mTOR, Igf1, Igf1R, and Rheb. Igf1R, Igf1 and Rheb mRNAs were all down-regulated in soleus muscle of HFD-fed mice for 12 weeks. Consistently with lower levels of Rheb, mTOR mRNA was increased $(\sim 1.8$-fold, $\mathrm{p}<0.05)$ in soleus muscle of these animals. Decreased myogenesis in skeletal muscle of obesity prone animals has been observed, thus we attempted to measure MyoD expression in soleus muscles of HFD mice. Indeed, MyoD mRNA was decreased in soleus muscles of HFD-mice for 8 weeks compared to the control group (control: $1.00 \pm 0.74 \mathrm{vc} \mathrm{HFD:} 0.46 \pm 0.17, \mathrm{p}<0.05$ ).

Conclusions: These findings reveal that reduced insulin sensitivity is associated to differential effect on the muscle-specific miRNA expression.

Financial support: FAPESP (2011/05876-6). 
P010

\section{Current Perspectives on Hyperhomocysteinemia and Alzheimer Disease: A Nutrigenomics Approach}

Amanda C.C.C. Carlos, Mariana Séfora B. Sousa, Michel Cardoso Angelis-Pereira

Sao Paulo University

Alzheimer's disease (AD) is a neurodegenerative process characterized by extracellular deposition of amyloid substance in senile plaques and cerebral vessels, which leads to progressive deterioration of cognitive functions. The causes of AD are not fully understood, though, it is known that the disease's development involves genetic and environmental factors. Thus, studies have linked hyperhomocysteinemia as a risk factor for the AD. Homocysteine is a methionine derived from an amino acid, participating in the folate/methionine pathway and is critically linked to DNA methylation and modified histone. The methylation is the addition of a methyl group in which cytosine usually precedes a guanine ( $\mathrm{CpG}$ dinucleotide), and is mainly present in promoter regions of genes. Methyl radical donors are obtained by diet, which are methionine, folate, vitamin B12 and choline. For DNA methylation, homocysteine must be converted to methionine (folate dependent reaction) which in turn is produced to S-adenosyl methionine (SAM), which substance is the methyl group donor DNA by the action of DNAmethyltransferases (DNMT), becoming SAH. The folate inadequacy and other B vitamins, avoid DNA methylation, and increased levels of homocysteine and S-adenosyl-homocysteine (SAH). High quantities of SAH inhibit the activity of methyltransferase activity and improve demethylases. Other researches have shown that the expose specific sites of presenilin (PSEN-1 genes and PSEN-2, encoding cell membrane proteins) increasing the activity gamma-secretase and producing amyloid $\beta$ peptide. Accumulation of this peptide is harmful to the neurons membrane, it promotes neurodegeneration and deregulating calcium channels and NGF (nerve growth factor) levels, and also blocking receptors for vitamin D. Furthermore, studies points out the NF- $\kappa B$ as transcription factor activated in neurodegenerative processes such as in $\mathrm{AD}$. In addition, current studies have pointed out the importance of some polymorphisms in $\mathrm{AD}$, such as the MTHFR C677T (characterized by reduced activity of the methylenetetrahydrofolate reductase enzyme), MTHFD1 G1958A (associated with increased SAH) and C $\beta$ S 844ins68 (which decreases the performance of cystathionine beta-synthase, an enzyme converting homocysteine to cystathionine). Despite of it has strong evidence presented between hyperhomocysteinemia and $\mathrm{AD}$, papers have still been inconclusive, which indicates the need for further studies in different populations.

\section{P011}

Oxidative and Inflammatory Processes Involved in Atherosclerosis: A Molecular Approach

Mariana Séfora B. Sousa, Amanda C.C.C. Carlos, Michel Cardoso De Angelis-Pereira

Sao Paulo University

Atherosclerosis is characterized by narrowing of the inner diameter of blood vessels. It is related to diverse physiologic conditions, including hypercholesterolemia, specially increase of low density lipoproteins (LDLs). Important advances in the comprehension of atherosclerosis mechanisms provided evidence that LDL oxidation and secretion of inflammatory mediators are key elements in the initiation and progression of atherosclerosis. LDL become atherogenic after undergoing oxidation by myeloperoxidase, lipoxygenase and reactive oxygen species, that transform them into oxidized LDL (highly bioreactive and cytotoxic). In response to injury, the endothelium expresses adhesion molecules for monocytes such as intercellular adhesion molecule (ICAM-1) and vascular cellular adhesion molecule (VCAM-1), which promotes rolling and adhesion of these cells in the vessel. Once attached, the monocytes migrate to the internal layer of the artery in response to chemotactic stimulus, meanly monocyte chemotactic protein (MCP-1), those differentiate into macrophages, when internalized the LDL oxidized form the foam cells. Several biological effects of oxidized LDLs are mediated through changes in the activity of transcription factors and subsequently in gene expression. Research has shown that ox-LDLs decrease the expression of ATP-binding cassette transporter-1 (ABCA1) by inhibiting liver $\mathrm{X}$ receptor (LXR) activation in endothelial cells, which may contribute to endothelial dysfunction and plaque formation. Both oxidized LDL and the products of oxidation, bind to scavenger receptors and the toll-like receptor (TLR) located in the macrophages stimulating the secretion of IL-1 $\beta$ and TNF- $\alpha$. Also, they activate NF- кappa B (NF-kB) pathway and the activator protein-1 (AP-1), which activates transcription of genes encoding adhesion molecules, chemokines, eicosanoid and inflammatory cytokines. Furthermore, ox-LDL modulates peroxisome proliferator-activated receptor- $\alpha$ (PPAR- $\alpha$ ) and PPAR- $\gamma$. On the other hand, it has been demonstrated that the nuclear factor erythroid (Nrf2), a major factor that activates transcription of genes encoding proteins antioxidant, is activated under conditions of extreme stress and can be a protective agent of the arteries, through the negative regulation of MAPK (mitogen-activated protein kinases), mainly MKK 3 and 6 . A better understanding of molecular mechanisms may contribute to the development of new alternatives for the treatment of atherosclerosis. 


\section{P012 \\ Participation of TLR4 Receptor in the Inflammatory Status in High-Fat Diet- Induced Obesity: Possible Modulating Effect of Omega-3 Fatty Acids}

\author{
Amanda Rabello Crisma, Laureane Nunes Masi, \\ Cátia Lira Amaral, Amanda Roque Martins, \\ Marco Aurélio Ramirez Vinolo, Rui Curi \\ University of São Paulo
}

Obesity is a worldwide problem and the main trigger of chronic inflammation that accompanies type 2 diabetes mellitus (DM2) and insulin resistance. Since TLR4 receptor is involved with inflammatory response in many tissues and omega- 3 fatty acids are considered anti-inflammatory agents, the aim of this study was to characterize the role of TLR4 receptor in the inflammation caused by feeding a high fat diet and to evaluate the effect of supplementation with fish oil. Wild-type and TLR4 knockout (TLR4 ${ }^{--}$) C57BL/6J adult male mice received control diet (CD, $9 \%$ of energy from fat) or high-fat diet (HFD, 59\% of energy from fat) for 8 weeks. Supplementation with fish oil began 4 weeks prior to induction of obesity and was kept until the end of the protocol. Control animals were supplemented with water. It was assessed body weight and food intake, responsiveness to insulin, adipokine production by fat tissue and cytokine, reactive oxygen species (ROS) and nitric oxide (NO) production by peritoneal macrophages. Wild-type animals fed a HFD showed increase of leptin production, but no change in the production of other adipokines. Fish oil supplementation of animals fed a HFD did not prevent the increase of leptin. HFD led to increase of TNF- $\alpha$, IL-1 $\beta$, IL-6, NO and ROS production and decrease of IL-10 production. Production of IL-1 $\beta$, IL- 6 and NO was normalized with fish oil supplementation. Macrophages from TLR $4^{-/}$animals fed a CD had lower production of cytokines and NO after stimulation with LPS than wildtype animals fed the same diet. There was no alteration in production of NO, TNF- $\alpha$ and IL-10 but an increase in IL-1 $\beta$ and IL- 6 production by $\mathrm{TLR}^{-/}$animals fed HFD. However the increase of IL- $1 \beta$ and IL-6 was lower than that observed in wild-type animals fed the same diet. After supplementation with fish oil, TLR $4^{-/}$animals fed a HFD showed decrease in the production of NO and IL-1 $\beta$. The results show that HFD leads to an inflammatory status with increase of proinflammatory cytokines, NO and ROS production and decrease of IL-10 production. This alterations were partially prevented in animals lacking TLR4. Supplementation with fish oil had a partially protective effect in both wild-type and TLR $4^{-/}$animals. The association of $\mathrm{TLR}^{-/}$and fish oil supplementation seems to have a positive effect in preventing chronic inflammation caused by HFD.

\section{P013 \\ Gene Expression in PBMC Is Inversely Correlated to Plasma Levels of IL-6 in Clinically Healthy Adults}

\author{
Ana Carolina Pinheiro Volp, José Luiz Marques Rocha, \\ Denise Machado Mourão, Caroline Müller, Kiriaque Barra \\ Ferreira Barbosa, Josefina Bressan \\ UFV, UFOP, UFS
}

Background: Peripheral biomarkers of metabolic diseases have been studied in order to identify tools which indicate a possible metabolic prognosis. Peripheral blood mononuclear cells (PBMC) have been used in gene expression studies by their metabolic activity. However, there are gaps about how the inflammatory process could lead to the development of chronic diseases in healthy individuals.

Objectives: To evaluate the gene expression in PBMC and the circulating levels of IL-6 in clinically healthy adults.

Methods: This study evaluated 92 women and 68 men whose ages ranged from 18 to 35 years. It was considered as exclusion criteria for evidence of any disease related to oxidative stress, chronic inflammation, changes in body composition or metabolism of nutrients. Blood collection was performed after fasting for 12 hours. Isolation of PBMC was accomplished by the method of gradient centrifugation (Polymorphoprep ${ }^{\circledR}$ ). The extraction of total RNA was made using Trizol ${ }^{\circledR}$ and its concentration and its purity were determined by spectrometer. The integrity of the extracted RNA was verified by agarose gel electrophoresis. The gene expression was performed using the method threshold cycle (TC), using the real-time PCR, with fluorescence detection SYBR Green. The expression of target genes was based on the comparative methods proposed by Zhao \& Fernald (2005), where the mean values of triplicate TC's of each sample were used to calculate the expression of the gene of interest in each individual group normalized by the average of two genes selected controls ( $\beta$-actin and GPDH). The level of IL-6 was performed by ELISA, in EDTA plasma. According to the distribution of variables the Student's t test or the Mann-Whitney-U test was adopted for comparison between the groups categorized by the median of the plasma concentration of IL-6. The software SAS version 9.0 was used to statistical analysis, and it was considered the $5 \%$ of probability.

Results: The blood concentration of IL-6 is higher in males (5.19 \pm 1.54 vs $3.45 \pm 1.51, \mathrm{p}<0.001)$, however, the same does not occur in gene expression, since it was also expressed in both sexes. The gene expression in PBMC has inversely correlated to the plasma concentrations of IL-6 $(p=0.03)$. No correlation was found between gene expression of IL-6 and lifestyle and body composition variables.

Conclusions: The contribution to the production of IL- 6 can be particularly small from PBMC in clinically healthy young adults.

Financial support: CAPES, CNPQ, FAPEMIG, UFV, UFOP. 
P014

An Aqueous Extract from Rosemary Reduces Concentrations of Reactive Oxygen Species and Increases HO-1, SOD-2 and GCLC mRNA Levels in HEPG2 Cells Under Hyperglycemic Conditions

\author{
Ana Mara Oliveira Silva, Joy Michelle Stanilka, \\ Cheryl Ann Rowe, Susan S. Percival, \\ Illana Louise Pereira Melo, Dalva A.Portari Mancini, \\ Jorge Mancini-Filho \\ University of Sao Paulo
}

Background: Hyperglycemia leads to oxidative stress and damage in cells. These effects are related to glucose toxicity. The mechanisms of glucose toxicity are associated with an increase in reactive oxygen species (ROS) and cell dysfunction. Recent studies have shown that phenolic compounds may reduce oxidative stress by modulating antioxidant defenses via the transcription factor Nrf2. Nrf2 is associated with the expression of genes involved in ROS production (HO-1), free-radical scavenging (SOD-2) and glutathione metabolism (GCLC).

Objective: To evaluate the effect of an aqueous extract (AE) of rosemary on cell viability of HepG2 cells under hyperglycemic conditions, ROS generation and expression of genes involved in antioxidant defense.

Methods: HepG2 cells were cultured under normal $(5.5 \mathrm{mM})$ or high glucose conditions $(25-00 \mathrm{mM})$, and treated with the $\mathrm{AE}$ of rosemary at different concentrations $(1-100 \mu \mathrm{g} / \mathrm{mL})$ for 24,48 and 72 hours. Cell viability, intracellular ROS generation and HO-1, SOD-2 and GCLC mRNA levels (qRT-PCR) were examined. Statistical significance was determined using the one-way analysis of variance followed by the Tukey post-test.

Results: Hyperglycemia affected cell viability and stimulated ROS generation in HepG2 cells, which coincided with decreased HO-1 and GCLC mRNA levels. Hyperglycemia, when associated with high concentrations of $\mathrm{AE}(100 \mu \mathrm{g} / \mathrm{mL})$, reduced cell viability. This effect was quite pronounced and was related to increased production of ROS. However, lower concentrations of AE $(1-50 \mu \mathrm{g} / \mathrm{mL})$ had no effect on cell viability and reduced ROS levels in a dosedependent manner. A $10 \mu \mathrm{g} / \mathrm{mL}$ concentration of AE significantly increased mRNA levels of HO-1, SOD-2 and GCLC in HepG2 cells exposed to $50 \mathrm{mM}$ glucose for 72 hours.

Conclusions: The phenolic compounds present in the AE of rosemary attenuated oxidative stress in HepG2 cells under hyperglycemic conditions, by increasing the expression of HO-1, SOD-2 and GCLC, genes involved in antioxidant defense, possibly via the transcription factor Nrf2.

Acknowledgments: National Council for Scientific and Technological Development (CNPq).

\section{P015}

Total Phenolic Compounds in Sample of Yerba-Mate (Ilex Paraguariensis St. Hil.)

\author{
Ana Ceolin Colpo, Hemerson Rosa, \\ Vanessa Brum Camargo, Felipa Melgarecho Bassante, \\ Maria Eduarda Lima, Andreas Mendes, Vanderlei Folmer \\ UNIPAMPA
}

Background: Ilex paraguariensis St.Hilaire (Yerba mate) is a South America native species. Dried and ground leaves are used in the preparation of a peculiar beverage, named "chimarrão", "maté" or "terere". This beverage has been acknowledged for its antioxidant capacity, and the polyphenols concentration has strong correlation with this function. Extracts of this beverage were used, as basis, to this study.

Aims: To analyze the variation in the content of total polyphenols from three herbal compounds marketed in Argentina.

Material and Methods: The herbal compounds were bought from a commercial establishment in Argentina. The brands analyzed were: El Chasqui, Yemaipe, Romance. The aqueous extracts were obtained mimicking the "mate" using a medium size gourd, yerba mate, "mate pump", Kitasato flask and a vacuum pump. The extracts of $1^{\circ}, 2^{\circ}, 5^{\circ}, 10^{\circ}, 15^{\circ}$ infusions (mates) were filtered, and stored in eppendorfs to subsequent analysis. The concentration of total polyphenols in the samples was determined by UV-visible spectrophotometry, using a modified Folin-Cioacalteau method. Data were subjected to analysis of variance, and means were compared using Tukey's test. Statistical significance was accepted at $p<0.05$.

Results: Chasqui and Yemaipe had the highest concentration of phenolics in the second mate and the Romance in the first. For the first two the decrease was statistically significant between the $2^{\circ}$ and $10^{\circ}, 2^{\circ}$ and $15^{\circ}$ mates, for Romance between $1^{\circ}$ and $10^{\circ}, 1^{\circ}$ and $15^{\circ}$ mates.

Conclusions: In the course of consumption there was a decrease in the amount of total polyphenols.

Financial support: FAPERGS, CNPQ, CAPES.

\section{P016 \\ Oxidative Stress and Antioxidant Profile in Leprosy Patients}

Anderson Marliere Navarro, Fabiana M. Oliveira, Alceu Jordão Junior, Norma T. Foss, Marco Andrey Frade

Faculdade de Medicina de Ribeirão Preto - USP

Background: Oxidative stress and the concomitant antioxidant depletion are important metabolic conditions observed in leprosy patients. The evaluation of these parameters may prevent the deleterious effects that hamper treatment and rehabilitation.

Methods: We analyzed 30 serum samples from adult patients with the multibacillary (MB) and paucibacillary (PB) forms of leprosy and 40 samples from a control group. The marker of lipid peroxidation malondialdehyde (MDA) was quantitated by the TBARS method proposed. Reduced glutathione (GSH) was quantitated by the 
Table 1. MDA, GSH and vit E values for the various clinical forms of leprosy and for the controls. Data are reported as mean

\begin{tabular}{llll}
\hline Groups & $\begin{array}{l}\text { TBARS } \\
\text { n(mean:min-max }) \\
(\mu \mathrm{M} / \mathrm{L})\end{array}$ & $\begin{array}{l}\text { GSH } \\
\text { (mean:min-max }) \\
(\mu \mathrm{M} / \mathrm{L})\end{array}$ & $\begin{array}{l}\text { Vitamina E } \\
\mathrm{n}(\operatorname{mean}: \min -\mathrm{max}) \\
(\mu \mathrm{M} / \mathrm{L})\end{array}$ \\
\hline Control & $(30) 5.9,2.0-13.5^{\mathrm{a}}$ & $(30) 374.5,214.5-807.0^{\mathrm{a}}$ & $(30) 13.9,8.5-24.8^{\mathrm{a}}$ \\
\hline $\mathrm{MB}$ & $(26) 13.5,5.9-22.8^{\mathrm{b}}$ & $(38) 312.1,145.9-564.5^{\mathrm{b}}$ & $(32) 3.9,1.11-11.3^{\mathrm{b}}$ \\
\hline $\mathrm{PB}$ & $(14) 14.7,9.2-33.3^{\mathrm{b}}$ & $(14) 392.0,174.5-629.5^{\mathrm{b}}$ & $(6) 4.6,1.3-7.0^{\mathrm{b}}$ \\
\hline a,b Kruskal-Wallis test $(\mathrm{p}<0.05)$ & &
\end{tabular}

method described by Sedlack \& Lindsay (1968). Vitamin E (vit E) was determined by high performance liquid chromatography. The data determined for the MB and PB groups and for the controls were compared by the Kruskal-Wallis test. When differences were detected between some group pairs, the data were analyzed by the Dunn posttest.

Results: Mean patient age was $44.6+8.9$ years and most patients were men $(71.4 \%)$. The mean age of the control group was $24+3.7$ years, and most subjects were women $(78.6 \%)$. Table 1 presents the values of the variables quantitated (in $\mu \mathrm{M} / \mathrm{L}$ ).

Conclusion: Leprosy patients have a high degree of oxidative stress and antioxidant depletion. In the present study it was possible to detect differences between forms of leprosy and for the controls. Nutritional intervention may be beneficial for the correction of this metabolic condition.

Financial support: FAPESP (2011/11704-3).

\section{P017 \\ Hepatic Steatosis Progression Was Not Associated with Cardiovascular Performance Alteration Under Chronic Nutritional Overload}

\author{
Andre Ferreira Nascimento, Renata A.M. Luvizotto, \\ Camila R. Correa, Katashi Okoshi, Aline O. Martins, \\ Tamiris A.S. Oliverira, Ana Lucia A. Ferreira, \\ Xiang Dong Wang, Antonio C. Cicogna \\ UNESP/TUFTS University
}

Background: Consumptions of higher energy-dense foods enriched in fat and sugars in Western countries have been associated with metabolic disorders, including obesity and non-alcoholic fatty liver disease (NAFLD). Recent evidences suggest that NAFLD is a risk factor for cardiovascular disease.

Objectives: To evaluate whether chronic nutritional overload might be a factor for hepatic steatosis progression and, consequently, cardiovascular performance alteration.
Methods: Male Wistar rats were randomly divided to receive either chow diet [C, $25 \%$ protein, $58 \%$ carbohydrate and $5 \%$ fat $(17 \%$ saturated and $82 \%$ unsaturated)] or high-fat diet [H, 21\% protein, $45 \%$ carbohydrate and $29 \%$ fat (50\% saturated and $50 \%$ unsaturated) plus sugar in the drinking water $(300 \mathrm{~g} / \mathrm{L})$ for 6,12 and 24 weeks. Adiposity index (sum of fat deposits normalized by body weight) and hepatic and plasma triacylglycerol and free fatty acid concentration were evaluated. Hepatic steatosis was analyzed by H\&E staining. Gene expression of hepatic metabolism-related gene [DGAT2, FAS, SREBP-1c and PPAR- $\alpha$ ] was assessed by Real Time PCR. Cardiovascular performance was analyzed by a two-dimensional echocardiography. Results were presented as mean \pm standard. Comparison between groups was performed by Two-Way ANOVA followed by post hoc Tukey test.

Results: The nutritional overload significantly increased adiposity index in all three experimental periods compared to $\mathrm{C}$ group. The higher amount of adipose tissue in $\mathrm{H}$ group was associated with elevated plasma TG concentration, while plasma FFA was influenced just with long-term of obesity. The nutritional overload resulted in a time-dependent hepatic steatosis progression in $\mathrm{H}$ group, which related to the alterations of SREBP-1c and FAS, but not PPAR-a and DAGT2. Hepatic TG and FFA concentration was elevated in H group than $\mathrm{C}$ group in all moments. These metabolic changes in the livers did not result in any dysregulation of cardiac morphology and function in $\mathrm{H}$ group compared with $\mathrm{C}$ group.

Conclusions: Chronic nutritional overload induced and promoted hepatic steatosis progression. However, this metabolic scenario did not alter cardiovascular performance evaluated by in vivo two dimensional echocardiography.

Financial support: FAPESP (2011/21664-9, 2011/09945-2). 


\section{P018 \\ Indicated for the Young Investigator Award \\ Moderate Alcohol Consumption Alters Hepatic Metabolism-Related Markers in Rats with Pre-Existing NASH}

\author{
Andre Ferreira Nascimento, Blanche Ip, \\ Renata A.M. Luvizotto, Xiang Dong Wang \\ UNESP/TUFTS University
}

Background: It has been shown that moderate consumption of alcohol in rats with pre-existing nonalcoholic steatohepatitis (NASH) increased hepatic inflammation.

Objectives: To investigate whether moderate alcohol intake alters hepatic lipid metabolism, SIRT1 expression and Adiponectin/ Adiponectin receptor (AdipoR)-related signaling in a pre-existing NASH status.

Methods: Sprague-Dawley rats were fed with a high-fat diet (HFD, 71\% energy from fat) for 6 weeks to induce NASH. Rats were then equally divided into 2 sub-groups: fed either a modified high-fat diet (HFD, 55\% energy from fat) or a modified high-fat alcoholic diet (HFA, 55\% energy from fat and 16\% energy from ethanol), for additional 4 weeks. Genes involved in hepatic lipid metabolism and Adiponectin/AdipoR-related signaling as well as SIRT1 protein were determined. Plasma adiponectin, triaciglycerol (TG) and free fatty acids (FFA) were analyzed. Hepatic TG and FFA concentration were also evaluated.

Results: In comparison to HFD group: 1) HFA significantly decreased hepatic AdipoR1 but not AdipoR2, 2) plasma adiponectin was lower in the HFA group although this change only reached a statistical tendency $(\mathrm{p}=0.06)$, and 3$)$ FAS gene expression, SREBP-1c and SIRT1 protein expression were significantly up-regulated, while DGAT1/2 and CPT-I gene expression were significantly down-regulated in HFA. Unexpectedly, HFA did not alter the phosphorylations of AMPK and ACC as well as PPARa protein level. In addition, there were no differences on plasma and hepatic TG and FFA concentrations between two groups.

Conclusions: These data suggest that the down-regulation of Adiponectin/AdipoR-related signaling may contribute to the moderate alcohol consumption-exacerbated liver injuries in rats with preexisting NASH.

Financial support: FAPESP / NIH / USDA (2011/21664-9 / R01CA104932 / 1950-51000-064S).

\section{P019}

Tributyrin Treatment Leads to Alteration in Gut Microbiota Composition and in GutAssociated Lymphoid Tissue

\author{
Andrea Caricilli, Angela Castoldi, Vinicius Andrade, \\ Enio Bassi, Marco Aurélio Vinolo, Tiago de Souza, \\ Susan Da Silva, Mario Saad, Rui Curi, Niels Câmara \\ University of São Paulo
}

Background: Low-grade inflammation, found in obese individuals, is associated with impaired intestinal barrier and increased lipopolysaccharide serum concentration. Obesity is also associated with alteration in gut microbiota composition. However, the mechanism by which altered bacterial composition may lead to increased gut permeability is not yet understood. Short-chain fatty acids, such as butyrate, are fermentation products of some types of bacteria and studies have shown that they may interfere with the modulation of the immune system.

Objectives: Our study aims to investigate the modulation of the immune system found in the gastrointestinal tract of diet-induced obese mice and correlate it with changes in intestinal barrier function after treatment with butyrate prodrug, tributyrin (TB).

Methods: C57BL/6 male mice fed a standard chow or high-fat diet (HFD) were treated with TB $(2 \mathrm{~g} / \mathrm{kg}$, by gavage) during 10 weeks and evaluated for body weight gain, glucose homeostasis, intestinal immune cell profile by flow citometry, gut microbiota composition by $16 \mathrm{~S}$ mRNA sequencing and intestinal tight-junction protein expression by Western Blot.

Results: TB-treated mice fed a HFD gained less weight and presented increased glucose tolerance than saline-treated mice. However, no significant differences were observed concerning food intake of TB-treated animal, neither in standard chow nor in HFD group. TB-treated mice on a HFD showed increased expression of T regulatory cells $(\mathrm{CD} 4+\mathrm{CD} 25+$ Foxp3 +$)$ and decreased TNF-alpha and IFN-gamma producing cells (TCD8 + and TCD4 + ) in mesenteric limph nodes. Moreover, TB-treated mice on a HFD showed increased expression of IL-17 producing cells in mesenteric limph nodes. After HFD feeding and TB treatment, alteration in the gut microbiota composition was also observed, with decrease in Firmicutes and increase in Verrucomicrobia phyla. Intestinal barrier was altered by TB treatment, with increase of tight-junction proteins, such as ZO-1 and occludin.

Conclusions: Our results suggest that TB treatment leads to an improvement of the intestinal barrier by reducing proinflammatory cytokine production and increasing inflammatory suppression by regulatory $\mathrm{T}$ cells. $\mathrm{TB}$ treatment also triggers changes in the gut microbiota composition, which might contribute to the modulation of the intestinal immune system. Thus, tributyrin may present itself as a potential drug to be used in the treatment of obesity.

Financial support: FAPESP (2011/19247-0). 


\section{P020}

\section{Camu-Camu Extracts (Myrciaria Dubia McVaugh) Inhibits ROS and iNOS in Lipopolysaccharide-Stimulated Mouse Macrophages}

\author{
Any Elisa Schmidt Gonçalves, Bruno Marcotte, \\ Marie Julie Dubois, Maria Inés Genovese, \\ Franco Maria Lajolo, Yves Desjardins, André Marette \\ Universidade de São Paulo
}

Inflammation is involved in numerous diseases, including chronic inflammatory diseases and the development of cancer. Macrophages play an important role in inflammation through the release of inflammatory mediators involved in the immune response. Inappropriate and prolonged macrophage activation is largely responsible for the pathology of acute and chronic inflammatory conditions. It has been already described that fruits bioactive compounds possess a variety of biological activities, including antifungal, antibacterial and antiinflammatory activities. Camu-camu is an Amazonia fruit with a high content of antioxidants, specially phenolics compounds and vitamin C. However, there is no information of the anti-inflammatory effects of camu-camu. A hydromethanolic extract (Crude Extract - CE) was prepared and fractionated by solid-phase extraction, providing two different fractions: Total Polyphenolics (TP) and Total Flavonoids (TF). Phenolic compounds of each fraction were identified and quantified by HPLC. Anti-inflammatory properties of CE $(500 \mu \mathrm{g} / \mathrm{mL})$, TP $(500 \mu \mathrm{g} / \mathrm{ml})$ and TF $(500 \mu \mathrm{g} / \mathrm{ml})$, were investigated using lipopolysaccharide (LPS)-stimulated J774.1 macrophages for 6 and 24 hours as in vitro model. As inflammatory parameters, nitric oxide (NO) production was evaluated by Griess reaction, as well as effects on inducible NO synthase (iNOS) expression on intracellular signaling pathways activation, which were analyzed by Western blot using specific antibodies. At the time of 6 hours of LPS stimulation, crude extract (CE) inhibited NO production and iNOS expression. Both TP and $\mathrm{TF}$ fraction significantly inhibited NO production, however they were not able to inhibit iNOS expression. At 24 hours of stimulation, CE was the most effective by inhibiting NO production, but none of three fractions were able to modulate iNOS expression. Our data support the usage of Camu-camu extracts as a natural source of antiinflammatory and antioxidants compounds.

Financial support: CNPQ.

\section{P021 \\ Offspring from Dams Fed High-Fat Diet During Pregnancy and Lactation Presented Activation of Endoplasmic Reticulum Stress}

Arine Mattos Melo, Rafaela Oliveira Benatti, Nicole Guimarães Ashino, Letícia Martins Ignácio-Souza, Janaína Cursino, Gustavo Aparecido Santos, Licio Augusto Velloso, Adriana Souza Torsoni, Marcio Alberto Torsoni

Faculdade de Ciências Aplicadas - Unicamp

Background: Obesity is associated to increase in the serum proinflammatory cytokines and activation of serine-kinases, as JNK and IKK, responsible for the impairment in insulin signaling. Obese subjects also present proteins related to unfolded protein response (UPR) activated, an indicator of endoplasmic reticulum stress and responsible by activation of JNK and IKK. Maternal obesity and the high fat diet consumption during pregnancy and lactation has been linked to metabolic damage in the offspring. However, the contribution of UPR to metabolic disorders in offspring of high fat fed mothers remains unknown.

Objectives: Evaluate the activation of UPR in the offspring of dams fed with high fat diet during pregnancy and lactation.

Methods: Females of Swiss mice were fed with either standard chow (SC) or high-fat diet (HFD) during gestation and lactation. After weaning the offspring was divided in SC-offspring (SC-O) and HFD-offspring (HFD-O) and both groups were fed with standard chow until the experiment period. The analyses were performed on the 28th day of life. Initially, we evaluated the body weight. The phosphorylation of the proteins PERK, JNK, eIF2 $\alpha$, in the liver and hypothalamus were analyzed through Western Blot technique. Relative gene expression of CHOP, GRP78, IL1 $\beta$, TNF $\alpha$ and IL10 were measured in the hypothalamus by real time PCR.

Results: No differences in body weight between the groups on day (d) 2 after birth, but from d7 the HFD-O showed an increase in body weight compared to SC-O. In both tissues from HFD-O the level of p.JNK were increased. Besides, the hypothalamus also showed increase NFkB expression and gene expression of proinflammatory cytokines (IL1 $\beta, \mathrm{TNF} \alpha$ ) and decrease of IL10, an antiinflammatory cytokine, compared to SC-O. The gene expression of CHOP e GRP78 was also higher in HFD-O compared to SC-O. In both tissues the level of p-PERK, p-eIF $2 \alpha$ in HFD-O were increased compared to SC-O mice. Besides, hypothalamic insulin-induced phosphorylation of AKT of HFD-O was reduced compared to SC-O.

Conclusions: Recently weaned offspring from high fat diet dam present activation of proteins related to UPR in liver and hypothalamus, suggesting that these tissues are under metabolic stress condition. This cellular response may be related to diminished activation of the AKT by insulin in the hypothalamus leading to damage to the signal of satiety and glucose homeostasis in the offspring contributing to the development of obesity in adulthood. 
P022

\section{Protein or Methionine Restriction Affects Dicer Expression and MicroRNA Processing in Adipose Tissue of Rodents}

Beatriz Alves Guerra, Carmen E. Perrone,

Thiago Martins Batista, Everardo Magalhaes Carneiro,

Marcelo Alves Mori

Universidade Federal de São Paulo

Background: Caloric restriction promotes beneficial health effects and prolongs lifespan in diverse species, from yeast to primates. Our group recently demonstrated that microRNA (miRNA) processing is progressively down-regulated with age across the evolutionary spectrum. In particular, this phenomenon is characterized by a decrease in Dicer expression in adipose tissue, and this can be prevented by caloric restriction by mechanisms that are not fully understood. Methionine restriction has been shown to promote beneficial health effects that mimic the effects of caloric restriction in rodents. Thus, we sought to analyze whether the restriction of dietary protein or, more specifically, methionine had an effect on the miRNA processing pathway (i.e. Dicer, Argonaute-2 and miRNAs) in adipose tissue of rodents.

Objectives: To identify how nutritional interventions (i.e. protein or methionine restriction) affect the miRNA processing pathway in adipose tissue of rodents.

Methods: Four-week old $\mathrm{C} 57 \mathrm{Bl} / 6$ male mice were subjected to protein restriction (PR) or ad libitum chow diet for 14 weeks. A subgroup of these mice were put on a high fat diet (HF) with 10 weeks of age and sacrificed after 8 weeks. Methionine restriction (MR) started with eight-week old Fischer 344 rats and was carried out for 12 weeks. We tested the expression of Dicer and Argonaute-2 (Ago2) by RT-qPCR and Western blotting. The expression of miR-125b and miR-145 was assessed by RT-qPCR.

Results: Our preliminary results indicate that PR resulted in a 12.8-fold increase in Dicer expression at the mRNA, but not at the protein level, in the subcutaneous white adipose tissue of mice. HF decreased Dicer protein levels and attenuated by $32 \%$ the effect of PR on Dicer mRNA expression in these mice. The expression of miR$125 \mathrm{~b}$ and miR-145 also increased with PR and PR followed by HF. To test whether MR could explain some of the phenotypes observed in calorie or protein restricted animals, we measured Dicer and miRNAs in fat of methionine restricted rats. MR resulted in a 5.2-fold increase in Dicer and a 1.6-fold increase in Ago2 in the epididymal white adipose tissue. Moreover, miR-125b increased by 2.2 -fold in the MR group in relation to the control group.

Conclusions: The effects of caloric restriction on the miRNA processing pathway can be mimicked at least in part by protein or methionine restriction.

Financial support: FAPESP (2012/07259-7 E 2010/52557-0).

\section{P023}

\section{Green Tea Attenuates Oxidative Stress in Heart Remodeling Induced by Myocardial Infarction}

\author{
Beatriz H.B. Lustosa, Diego F. Batista, Ana A.H. Fernandes, \\ Katashi Okoshi, Marcos Minicucci, Luiz Matsubara, \\ Leonardo Zornoff, Paula S.A. Gaiolla \\ UNESP - Faculdade de Medicina de Botucatu
}

Objectives: The objective of this study is to evaluate whether green tea attenuates cardiac remodeling after acute myocardial infarction.

Methods: wistar rats were allocated in 4 groups: C (control), GT (diet with green tea $0.25 \%$ ), MI (infarcted group) IGT (infarcted group with green tea $0.25 \%$ ). After 3 months, animals were submitted to echocardiography and analysis of oxidative strees. Two way anova and Holm Sidak tests were performed.

Results: Both infarcted groups showed the same infarction size $(\mathrm{p}=0.7)$. Echocardiographic data without interaction: left atrium/ weight (with MI $14.4 \pm 0.5$, without MI $10.7 \pm 0.6)(\mathrm{p}<0,001)$ (with GT 13.0 \pm 0.5 , without GT $12.1 \pm 0.6)(\mathrm{p}=0.2)$, and diastolic ventricle diameter/weight (with MI $21.2 \pm 0.6$, without MI 17.4 \pm 0.7$)(\mathrm{p}<$ $0.001)$ (with GT 19.1 \pm 0.6 , without GT $19.7 \pm 0.7)(\mathrm{p}=0.5)$. Systolic function: fractional area change (with MI $41.2 \pm 1.8$, without MI 70.8 $\pm 2)(\mathrm{p}<0.001)$ (with GT $56.1 \pm 1.8$, without GT $55.8 \pm 2)(\mathrm{p}=0.9)$. Echocardiographic data with interaction $(\mathrm{p}=0.04)$ E wave deceleration time (EDT) (C 48.5 \pm 2.9 , GT $45 \pm 2.6$, MI $35.3 \pm 2.5$, MIGT 43 $\pm 2.4)$. Oxidative stress data without interaction: hydroperoxide (HP) $(\mathrm{p}=0.28)$ (with MI 204, without MI 165.3) $(\mathrm{p}=0.002)$ (with GT 174.3, without GT 195) ( $\mathrm{p}=0.068)$, and glutathione peroxidase $(\mathrm{GSH}-\mathrm{PX})(\mathrm{p}=0.12)($ with MI 36.8, without MI 34.8) $(\mathrm{p}=0.43)$ (with GT 38.3, without GT 33.3) $(\mathrm{p}=0.06)$. Data with interaction catalase $(\mathrm{p}<0.001)(\mathrm{C} 67.9$, GT 45.8, MI 35.1, MIGT 52.3), superoxide dismutase (SOD) $(\mathrm{p}=0.002)(\mathrm{C} 0.64$, GT 0.76, MI 1.04, MIGT 0.88).

Conclusions: There was no reduction in the formation of HPX, but is numerically smaller. IGT decreases SOD and catalase increases. It may be because SOD has already been consumed and then increases catalase to try to hold the oxidative stress. There was no difference in GPX.

Acknowledgment: This work is supported by Fapesp.

\section{P024 \\ Effect of Methylglyoxal, Glyoxal and Resveratrol on Breast Cancer Cells MCF-7 Viability}

Betina Schmidt, Carolina Agostinho, Eliane Filaho de Oliveira

Universidade Federal do Rio de Janeiro

Background: Breast cancer is the second most common cancer worldwide. Since the discovery of high aerobic glycolysis in tumors, it has remained unclear how to exploit this in chemotherapy. During the glycolysis, metabolites are formed including glyoxal and methyl- 
glyoxal, Advanced Glycation End-products (AGEs) precursors. The AGEs promote oxidative stress, morphological and functional changes and increase inflammatory mediators becoming aggressive to the healthy cells, but potentially chemotherapeutic. Recent studies show that resveratrol, a polyphenol, has anti-inflammatory and antioxidants properties becoming a promising chemopreventive agent.

Objectives: This study aimed to verify the action of methylglyoxal, glyoxal and resveratrol, alone and in combination on viability of MCF-7 cells.

Methods: MCF-7 cells were grown in Dulbecco's Modified Eagle Medium supplemented and grown at $37^{\circ} \mathrm{C}$ in an atmosphere of $5 \% \mathrm{CO}_{2}$. On reaching 70-80\% confluency they were treated with different concentrations of resveratrol, glyoxal and methylglyoxal. Cell viability was determined by MTT reduction assay.

Results: IC 50 values for methylglyoxal, glyoxal and resveratrol were $3.1 \mathrm{mM}, 3.0 \mathrm{mM}$ and $133 \mu \mathrm{M}$, respectively. To examine a possible synergistic effect of the precursors of AGEs with resveratrol the treatments were associated. The association enhances the decrease of viable cells, demonstrating a synergistic effect of drugs.

Conclusions: Methylglyoxal and glyoxal have a detoxification system composed of two major enzymes, glyoxalase I and II. The cytotoxic action of glyoxals, found in this study, reinforces the findings reported in the literature with others cancer cells. Increasing speed of the glycolytic pathway in cases of cancer, there is an increase of glyoxals formation, which would, hypothetically, have a protective effect and induce cancer cells apoptosis, but the literature explained that cancer cells undergo an adaptation of glyoxalase I expression and can detoxify the glyoxals without undergoing cytotoxic injury. It was proved that curcumin minimize glyoxalase I gene expression, increasing the amounts of glyoxals, justifying the chemotherapy power of the polyphenol. It is possible that resveratrol has a similar action to curcumin and the synergistic effect of resveratrol and glyoxals, shown in this study, is related to decreased expression of glyoxalase I. Studies involving the expression of the gene and protein have been made by our group to confirm the hypothesis.

Financial support: CAPES, FAPEJ, CNPQ, Fundação do Câncer.

P025

\section{Resveratrol Stimulates the Production of Reactive Oxygen Species in Breast Cancer} Cells

Patrícia Severo Ramos, Paula Seixas Costa, Jerson Lima Silva, Eliane Fialho

UFRJ

Background: It is estimated that in 2020, the worldwide incidence of cancer is on the order of 15 million, of which $60 \%$ of new cases will occur in developing countries. The breast cancer is the second most deadly cancer among women. Resveratrol, a phytoalexin found in grapes and red wine has been reported to exhibit a wide range of pharmacological properties and is believed to play a role as anti-cancer agent but the precise mechanism of action, however, is not fully elucidated. In the present study, the anticancer activity of resveratrol towards MCF-7 human breast cancer cells was investigated.

Materials and Methods: One breast cancer cell line (MCF-7) was cultured using standard techniques. Cell viability was determined by MTT assay. In order to demonstrate the decrease of the cell viability towards the production of reactive oxygen species (ROS), the cells were treated with resveratrol $(\mathrm{RV})(200 \mu \mathrm{M})$ for $24 \mathrm{~h}$. The generation of ROS was measured by using the cell-permeable nonfluorescent compound H2DCF-DA. N-Acetyl-L-cysteine (NAC) was used in different concentrations $(0-5 \mathrm{mM})$ for $24 \mathrm{~h}$ to evaluate the capability of reverse the pro-oxidant effect of RV $(200 \mu \mathrm{M})$, the same was done using L-Glutathione reduced (GSH) in different concentrations (10mM-300mM).

Results: RV inhibited cellular proliferation in time and dosedependent manner with an IC50 of $238 \mu \mathrm{M}$ and $151 \mu \mathrm{M}$ for 24 and 48 $\mathrm{h}$, respectively. ROS production was increased when MCF-7 cells were treated with RV $200 \mu \mathrm{M}$ for $24 \mathrm{~h}$. Recently, the effects of several drugs that produce ROS and interfere directly with apoptosis in cancer cells such as MCF-7 cells have been described. NAC and GSH completely reverted the ROS generation, promoted by high concentrations of RV, demonstrating a pro oxidant effect of RV which caused the decrease of the MCF-7 cell viability.

Conclusions: Taken together, our results demonstrate that RV inhibited the growth of MCF-7 cells in a dose-dependent manner and that this reduction in cell viability resulted from ROS generation which was reverted using different concentrations of NAC and GSH.

Financial support: CAPES, FAPEJ, CNPQ, Fundação do Câncer.

P026

\section{The Advanced Glycation End-Products (AGES) and the Complications of Diabetes Mellitus - A New Therapeutic Target for Nutrition}

\author{
Betina Schmidt, Eliane Fialho de Oliveira, \\ Carolina Agostinho \\ UFRJ
}

Diabetes mellitus is a significant concern in prevalence worldwide. A possible mechanism of tissue damage induced by hyperglycemia in Diabetes Mellitus is the formation of advanced glycation end-products (AGEs). The pathological effects of AGEs are related to its ability to modify the chemical properties and functions of various biological structures. During the Maillard reaction, some intermediate products are formed such as dycarbonils and $\alpha$-oxaldehydes as glyoxal, methylglyoxal and 3-D-glyoxuglucosone, precursos of AGEs. The intracellular increase in oxidative stress also contributes to the formation of AGEs. The preparation of food containing high fat and sugars at high temperatures $\left(130{ }^{\circ} \mathrm{C}\right)$ is the main exogenous source of AGEs. It is known that approximately 10\% of AGEs ingested with diet is absorbed although the mechanisms pertaining to such absorption are not fully elucidated. The fraction absorbed, about two thirds are retained in the body and one third is excreted in the urine, within 48 hours, by individuals with normal renal function. There is evidence that dietary AGEs are added to the pool of endoge- 
nous AGEs, favoring the emergence and progression of several complications of diabetes mellitus. Some anti-AGEs substances have been identified as $\mathrm{N}$-acetylcysteine and penicillamine. Polyphenols acts as hostages of methylglyoxal and glyoxal, precursors of AGEs, and may act as a new target for prevention of diseases associated with diabetes mellitus. Understanding the contribution of AGEs to the development of complications of Diabetes Mellitus will show new lines of research in medicine and functional foods in order to inhibit the formation or absorption of AGEs.

Financial support: CAPES, FAPEJ, CNPQ, Fundação do Câncer.

P027

\section{Vitamin A Modulation of IL-10 and IL-17 in Human CD4+ Cells Stimulated with Soluble Leishmania Infantum Antigens}

\author{
Bruna Leal Lima Maciel, Francisco P. Freire Neto, \\ João Firmino R. Neto, Tatjana Keesen, \\ Selma Maria B. Jerônimo
}

Universidade Federal do Rio Grande do Norte

Backgound: Vitamin A supplementation has long been used in developing countries to prevent mortality by diarrheal and respiratory diseases. Infection by Leishmania infantum is endemic in Northeast Brazil and may cause a spectrum of clinical outcomes that ranges from asymptomatic self-healing infection to visceral leishmaniasis (VL), a disease that is fatal even with treatment. VL is characterized by hepatosplenomegaly, fever, pancytopenia and altered hepatic function with increase in acute phase proteins and decrease in albumin synthesis. Determinants of the disease severity are not clearly understood, but nutritional status and the immune response influence the outcome of infection and disease. Cytokines as IL-10 and IL-17 are associated with parasite persistence/disease and protection against infection, respectively. IL-10 is a suppressive cytokine produced by effector CD4 + cells and T regulatory (Treg) cells. IL-17 is produced by inflammatory CD4 + (Th17) cells.

Objectives: This work analyzed the effect of retinoic acid (RA), an active vitamin A metabolite, and soluble Leishmania infantum crude antigens (SLA) in CD4 + cells isolated from healthy children.

Methods: PBMCs isolated from children $(n=9)$ with no signs of Leishmania infection (negative antibody to SLA and negative Montenegro skin test) were stimulated with SLA $(10 \mu \mathrm{g} / \mathrm{mL})$ or RA $(0.25 \mathrm{nM})$ or SLA $(10 \mu \mathrm{g} / \mathrm{mL})$ and RA $(0.25 \mathrm{nM})$. Cells were cultured for 20 hours and the production of IL-10, IL-17 in effector CD4 + cells and IL-10 in Treg cells (CD4 + CD25highFOXP3 + cells) was analyzed by flow cytometry.

Results: PBMCs stimulates in presence of RA + SLA increased the expression of CD25 in CD4 + cells, more than SLA alone $(32.4 \%$ and $22.6 \%$, respectively, $p=0.006)$. RA elevated the production of IL-10 in CD4 + cells more than SLA (4.1\% and 2.5\%, respectively, $\mathrm{p}=0.036)$. SLA stimulated IL-17 production by CD4 + CD25+ (6.2\% vs $3.4 \%$ without any stimulation), while RA addition down regulated IL-17 production to $2.8 \%$ of CD4 + CD25 + cells $(\mathrm{p}=$ $0.015)$. Stimulation by either SLA or RA + SLA did not result in significant changes in Treg IL-10.
Conclusions: Data show increased production of IL-10 and decreased IL-17 in CD4 + cells, which may suggest negative modulation of the immune response by vitamin A in presence of Leishmania antigens.

Financial support: National Institutes of Health, CNPQ.

\section{P028 \\ Arg213Gly Polymorphism in EC-SOD Gene and Its Relationship with Cardiovascular Disease}

\author{
Bruna Zavarize Reis, Graziela Biude Silva, \\ Isabela Saraiva Almeida, Silvia Maria Franciscato Cozzolino \\ University of São Paulo
}

Superoxide dismutases (SOD) are considered the first line of antioxidant enzyme defense systems. They are responsible for dismutation of superoxide anions to hydrogen peroxide, which in turn is catalyzed in water by catalase, glutathione peroxidase and peroxiredoxins. There are three isoforms of this enzyme in mammals: copperzinc (CuZnSOD, SOD1), found in the cytosol, manganese (MnSOD, SOD2), found almost exclusively in mitochondria, and the extracellular superoxide dismutase (EC-SOD, SOD3) in the extracellular space and fluids. EC-SOD is highly expressed in blood vessels, lung, kidney and uterus. In vascular tissue, EC-SOD is synthesized by vascular smooth muscle cells and fibroblasts, being located on the surface of the endothelial membrane bound to sulfated glycoproteins such as heparan sulfate and heparin. EC-SOD is the main arterial SOD isoform and thus makes a major contribution to cardiovascular defense. Some individuals may exhibit extremely high plasma levels of EC-SOD as a result of a SNP in the enzyme gene, the Arg213Gly. The polymorphism is located in the heparin binding domain, causing a decrease in affinity for glycosaminoglycans. As a result, there is an increase 10 to 30 -fold plasma concentration of SOD3 in polymorphic subjects. Consequently, despite high plasma concentration, the arterial wall may be deficient in EC-SOD and thus may have insufficient antioxidative capacity against superoxide anions and LDL oxidation, which could lead to increased atherogenesis. In fact, it was shown that heterozygous individuals have an increased risk of developing ischemic heart disease. In patients undergoing angiography, there was an association between the polymorphism and the severity of coronary artery disease and the risk for myocardial infarction. Polymorphic patients with diabetes and end-stage renal disease had significantly higher incidence of ischemic heart disease and cerebrovascular disease. In another study, uremic patients who carried the variant allele showed accelerated progression in renal failure and atherosclerosis. It was also observed a positive correlation between the presence of the polymorphism and severity of macro and microvascular complications in both diabetic and healthy subjects. Studies suggest that the presence of Arg213Gly variant in EC-SOD gene reduces the protective role of the enzyme in vascular wall, constituting an independent risk factor for cardiovascular disease. 


\section{P029}

\section{Effects of n-3 Polyunsaturated Fatty Acids in Treatment of Hypertriglyceridemia in Subjects with Diabetes Mellitus}

Caio E.G. Reis, Karla C. Ladim, Aline C.S. Nunes, Jane Dullius

University of Brasília

Diabetes mellitus increases the risk of hypertriglyceridemia and is an independent risk factor for cardiovascular diseases. Current literature reveals the beneficial effects of $n-3$ polyunsaturated fatty acids (n-3 PUFA) in hypertriglyceridemia treatment, however its efficacy for subjects with diabetes mellitus are still debatable. This literature review discusses the effects of n-3 PUFA supplementation in the treatment of hypertriglyceridemia and its effects on glycemic control in subjects with diabetes mellitus (type 1 and 2). A search was conducted in the LILACS, MEDLINE, and COCHRANE databases. Eighteen articles (fourteen clinical trials and four review studies) were selected for this review. All articles showed triglycerides reductions ranging from 18 to $35 \%$ (final vs. initial values) and 15 to $29 \%$ (placebo vs. intervention values). Furthermore the articles showed a reduction between $26-36 \%$ in very low-density lipoprotein and $5-22 \%$ in low-density lipoprotein, and an increase of 5-19\% in highdensity lipoprotein levels. There was no significant alteration of total cholesterol levels in most of the articles $(86 \%, n=16)$. The articles that evaluated fasting plasma glucose and glycated hemoglobin showed an increase of $18 \%$ and $16 \%$ respectively. These findings provide preliminary evidence that n-3 PUFA supplementation can be a promising tool in the treatment of hypertriglyceridemia in diabetes mellitus subjects. However may cause a slight increase in glucose and glycated hemoglobin levels. Further clinical trials are needed to investigate the complex mechanisms relating n-3 PUFA to the reduction of triglycerides levels and to understand its effects on lipid profile and glycemic control in people with diabetes.

\section{P030}

\section{Effect of Fish Oil Supplementation on Neutrophil Death in Wheelchair Basketball Players}

Camila Garcia Marques, Adriana Cristina Levada-Pires, Vinicius Coneglian Santos, Sâmia Rocha Alves, Rui Curi, Tânia Cristina Pithon-Curi, Maria Fernanda Cury-Boaventura

\section{Universidade Cruzeiro do Sul}

Background: Individuals with spinal cord injuries commonly develop infections of the urinary tract, respiratory tract, and skin, indicating that their host defense mechanisms may be diminished. The engaging of paraplegics in sports programs combined with an adequate food plan can minimize the risk of related diseases. Evidence suggests that fish oil rich in $n-3$ polyunsaturated fatty acids ( $n-3$ PUFA) has a beneficial effect on immune response.
Objectives: We investigated the effect of fish oil supplementation on neutrophil death in athletes with spinal cord injuries at rest and after basketball play.

Methods: Nine paraplegic athletes were supplemented with $3 \mathrm{~g}$ of fish oil per day. The athlete's blood samples were collected on rest and immediately after basketball play (60 minutes) before and after supplementation. Neutrophils were isolated by density gradient, using Histopaque-1077. The following determinations were carried out: loss of membrane integrity to evaluate necrosis and DNA fragmentation, phosphatidylserine externalization to evaluate apoptosis.

Results: Were studied nine athletes with following characteristics: age: $33.8+2.7$ years, height: $170+4 \mathrm{~cm}$, body fat: $22+5 \%$, BMI $23.9 \pm 3.16 \mathrm{~kg} / \mathrm{m}^{2}$ and training time: $6.9+5.4$ years. Basketball play induced an increase in the percentage of necrotic neutrophils $(2.57+0.37$ to $17.5+3.5 \%)$ but had no effect on percentage of cells with DNA fragmentation and phosphatidylserine externalization. Fish oil supplementation prevents the necrosis induced by training but induced a slight increase in the percentage of apoptotic neutrophils $(2.91+1.01$ to $5.42+1.47 \%)$.

Conclusions: Basketball play induced neutrophil death in wheelchair athletes by necrosis. Fish oil supplementation may be a strategy for basketball players in wheelchairs to promote improves in athletic performance and health for paraplegia condition.

Financial support: FAPESP (2010/04440-7).

\section{P031 \\ Polyphenol-Rich Cagaita (Eugenia Dysenterica DC) Extract Promotes Pancreatic Beta Cell Proliferation and Improves Insulin Sensitivity in Diabetic Rats}

\author{
Camilo Lellis-Santos, Laila Romagueira Santos, \\ Any Elisa Souza Gonçalves, Lucas Carminatti Pantaleão, \\ Ingrid Candido Garofolo, Marcela Roquim, Renata Gorjão, \\ William Tadeu Festuccia, Franco Maria Lajolo, \\ Maria Inés Genovese, Silvana Bordin, Rui Curi \\ University of São Paulo
}

Pancreatic beta cells failure and apoptosis are key events in the development and progression of Diabetes Mellitus (DM). Many bioactive compounds from natural sources have been described to delay or prevent DM through inhibition of oxidative stress and inflammatory processes in metabolism-related tissues, such as liver, muscle and adipose tissue. However, there is no data addressing the effects of phenolic extracts from the native Brazilian fruit Cagaita (Eugenia dysenterica DC) over pancreatic beta cell physiology. Pancreatic beta INS1e cells were treated with different concentrations $(100,500$ or $1000 \mu \mathrm{g} / \mathrm{mL}$ ) of polyphenol-rich Cagaita extract (PCE). PCE were also given to control and streptozotocin (SZT)-induced diabetic rats by gavage at dose of $3 \mathrm{~g} / \mathrm{Kg}$ body weight for 30 days. Phosphorylation of ERK1/2 was significantly increased after treatment with 100 and $500 \mu \mathrm{g} / \mathrm{mL}$, but the lower dose induced more effectively cell cycle progression and beta cell proliferation, measured by flow cytometry and $[3 \mathrm{H}]$-thymidine incorporation, respectively. Treatment of INS1e cells with $100 \mu \mathrm{g} / \mathrm{mL}$ of PCE for $24 \mathrm{~h}$ increased gene expression of proliferating cell markers (mcm7 and CDK4), and pre-incubation 
with ERK1/2 inhibitor UO126 for $1 \mathrm{~h}$ abrogated this effect $(\mathrm{P}<0.05)$. Among the seven phenolic compounds identified by HPLC, only quercetin significantly increased both phosphorylation of ERK1/2 and Akt at levels of PCE. And quercetin and the polyphenol mixture of all compounds at dose equivalent to PCE $(100 \mu \mathrm{g} / \mathrm{mL})$ were able to partially reproduce the effects of PCE on pancreatic beta cell proliferation. Control rats gavaged with PCE showed increased insulin content and mRNA expression in isolated islets, as well as Cyclin D1 expression, a mark of cell proliferation, when compared to salinetreated animals. Besides, islets from PCE-treated animals were more resistant to apoptosis induced by serum deprivation. PCE treatment was not able to counteract streptozotocin (SZT)- induced pancreatic beta cell death. Nevertheless, diabetic rats treated with PCE showed improved glycemia, insulin sensitivity, plasma antioxidant capacity and lipid profile when compared to diabetic rats. Cagaita extract induces pancreatic beta cell proliferation in an ERK1/2 and Akt dependent manner, and quercetin was the major compound to induce these effects. The extract also ameliorates SZT-induced damages in rats. Thus, PCE should be considered as new nutraceuticals to prevent or manage DM.

Financial support: FAPESP (2010/10778-0).

\section{P032 \\ Nutrition in the Post Genomic Era: Genetic Aspects Related to Obesity}

\section{Carlos Henrique Miotto \\ Faculdades Integradas ASMEC}

Obesity has reached epidemic proportions worldwide, with more than one billion adults being overweight, and is a major contributor to the global burden of chronic disease and disability, affecting virtually all ages and socioeconomic groups. The completion of the ambitious Human Genome Project in April 2003, has the ability for the first time, be held to read the complete model of the genetic makeup of a human being. The post-genome Era resulted in the development of several new "omics" technologies, applicable in many areas of science. Defined as nutritional genomics or nutrigenomics, this area aims to provide an understanding on how nutrition can affect the balance between health and disease by altering the expression or genetic structure of an individual. No human, except for identical twins have the same genome than others. Each charge is a set of genetic polymorphisms that makes them unique. The metabolism of drugs and nutrients is associated with the polymorphic profile of an individual, which may explain the adversity of reactions and responses to pharmacological interventions and nutritional. Like other chronic diseases, obesity is caused by multiple molecular pathways. Thus, except in special behavioral conditions, it was noticed that obesity usually results from small and continuous accumulation of body fat over the years due to changes in dietary habits and increased sedentary lifestyle associated with the polymorphism of several genes. Studies of monogenic and rare forms of human obesity may provide important information regarding endocrine physiology and the most common forms of obesity. In 2007, an association of SNPs - known as Fat Mass and Obesity-Associated (FTO) - the genetic regions linked to $\mathrm{BMI}$ and the risk of obesity, was identified in various populations, becoming the first locus associated with adiposity. The nutrigenomics will be the driving force of the future of nutritional research and will entail a major impact on public health.

\section{P033 \\ Effects of Maternal Plane of Nutrition and Weaning Age of the Offspring on Total RNA Yield in Subcutaneous Adipose Tissue}

\author{
Fernanda Trindade Rosa, Sonia Moisa, Johan Osorio, \\ Carolina Bespalhok Jacometo, Eduardo Schmitt, \\ Marcio Nunes Corrêa, Juan J. Loor \\ University of Illinois
}

Background: One of the carcass traits that influence meat quality is the intramuscular fat deposition which begins its development before birth. Therefore, maternal nutrition can potentially result in alterations of the development not only of the offspring but of adipose tissue including changes in gene expression. Overall, assess the RNA yield from tissue samples can generate important information for the gene expression analysis.

Objectives: Evaluate the total RNA yield in fat tissue from offspring of beef cows that were on different planes of nutrition during late gestation.

Materials and Methods: Thirty six cows at late gestation $( \pm$ 90 days from expected calving) were allocated into three planes of nutrition: 1) high plane of nutrition, fed with endophyte-infected tall fescue (F)/red clover pastures (C) plus $9.1 \mathrm{~kg}$ of a diet based on Distiller's Dried Grains with Solubles (DDGS) and soyhulls, 2) medium plane of nutrition, fed with F/C, $2.3 \mathrm{~kg}$ of DDGS and soyhulls, and 3) low plane of nutrition, fed with $\mathrm{F} / \mathrm{C}$ without supplementation. The offspring from each plane of nutrition were separated into an early weaning $(\mathrm{EW}, 79 \pm 2$ days of age) $(n=7)$ group housed in a feedlot, and a normal weaning $(\mathrm{NW})(\mathrm{n}=7)$ group in which calves remained with the dams until $186 \pm 2$ days of age. Biopsies from the tail fat pad were harvested at EW and NW and at the mid-point during the finishing phase. RNA was extracted from approximately $100 \mathrm{mg}$ of tissue using the Ambion miRNA/total RNA isolation kit as described by the manufacturer. The spectrophotometric absorbance at 260 and $280 \mathrm{~nm}$ was used to determine RNA quality, concentration and total yield. Statistical analysis was performed using the Proc Mixed Procedure in SAS, and a value of $\mathrm{P}<0.05$ considered significant.

Results: At the point of EW the total RNA yield ( $\mu \mathrm{g} / \mathrm{mg}$ tissue) was greater $(\mathrm{P}<0.0001)$ for offspring from dams in the high plane of nutrition than the medium and low planes $(119 \mu \mathrm{g}, 66 \mu \mathrm{g}$ and $42 \mu \mathrm{g}$, respectively). In addition, offspring from dams in the high plane of nutrition had greater $(\mathrm{P}=0.0009)$ total RNA yield $(116 \mu \mathrm{g})$ at the moment of NW than the medium and low planes (75 $\mu \mathrm{g}$ and $63 \mu \mathrm{g}$, respectively).

Conclusions: The maternal plane of nutrition during late gestation appears to affect the total RNA content of subcutaneous adipose tissue in the offspring. 


\section{P034}

\section{Prenatal Exposure to Fatty Acids and Its} Influence on the Developmental Biology

Fernanda Trindade Da Rosa, Carolina Bespalhok Jacometo, Eduardo Schmitt, Juan J. Loor, Marcio Nunes Corrêa

Universidade Federal de Pelotas

Dietary exposure during pregnancy could influence the offspring development. The maternal diet could cause changes in the offspring health and growth, these changes provided by molecular mechanisms and the effects could be across generations. The effects of a fetal programming can be multigerational, if the individual had a direct exposure to the nutrition or transgenerational if the effects are maintained throughout generations that were not directly exposed. DNA methylation and histone modifications are genetics processes that can regulate the gene expression. Based on environmental factors, like nutritional prenatal exposure, these alterations on gene expression potentially could cause alterations on the growth and on the metabolic status of the progenies. Approaches have focused on the benefits of fatty acids by maternal nutrition. Among the fatty acids, mammal requires essential ones, the polyunsaturated fatty acids (PUFAs), like linoleic (C18:2) and alpha linolenic (C18:3), which cannot be synthesized by the mammalian organism, therefore they must be obtained from the diets. These fatty acids are involved on the regulation of lipid metabolism, cell growth and differentiation and immune function. The beneficial effects of C18:2 and C18:3 on fetal development and on the offspring metabolism are well documented. As the main biology function of the PUFAs is to provide energy, researches demonstrated that these fatty acids can regulate the lipid metabolism due the activation of target transcription factors that stimulates fatty acid oxidation and/or inhibits the lipogenesis. Also, studies have demonstrated that the ratio between $\mathrm{C} 18: 2$ and $\mathrm{C} 18: 3$ are important for maintenance of polyunsaturated fatty acids homeostasis and for liver enzymatic control. In addition, observations showed that the metabolites of fatty acid desaturation, like arachidonic acid and docosahexaenoic acid are involved with the activity of brain membranes. However, there are studies with alpha linolenic acid that showed an anti-inflammatory effect avoiding some chronic diseases on the adult health. In the context of healthy development, the maternal diet with polyunsaturated fatty acids might be an important genetic agent within the adult life.

\section{P035 \\ Indicated for the Young Investigator Award \\ Regulation of Lipid Metabolism Transcription Factors by Polyunsaturated Fatty Acids on the Postpartum Period of Successive Generations in Rats}

Carolina Bespalhok Jacometo, Fernanda Trindade Da Rosa, Patrícia Mattei, Eduardo Schmitt,

Luiz Francisco Machado Pfeifer, Augusto Schneider,

Nelson José Laurino Dionello,

Francisco A. Burkert Del Pino, Marcio Nunes Corrêa

Universidade Federal de Pelotas

Background: Polyunsaturated fatty acids (PUFAs) nutrition exposure during fetal life could represent an important factor related to the offspring metabolic status.

Objectives: To evaluate the expression of transcription factors related to lipid metabolism by essential fatty acids and their hepatic profile through consecutive generations.

Methods: Eighteen female Rattus norvegicus - Wistar/UFPel, 8 weeks old, were separated in two groups: 1) diet rich in omega-6, with soybean oil (CTL) and, 2) diet rich in omega-3, with flaxseed oil (OM). The diets were elaborated in accordance with AIN-93 recommendations. After $30 \mathrm{~d}$ of adaptation the rats were mated. The males received only the control diet. The F1 females were selected at weaning ( $n=16 /$ group) and divided into three groups: females from OM group that continued receiving the same diet (OM-OM), females from $\mathrm{OM}$ group that began to receive diet containing soybean oil (OM-CTL), and females from CTL group that continued to receive the same diet (CTL-CTL). These animals were mated at $60 \mathrm{~d}$ old as described (G0). The F2 ( $\mathrm{n}=16$ /group) was selected as F1 and the same groups were maintained: OM-OM-OM, OM-CTL-CTL, and CTL-CTL-CTL. The F2 was mated as F1. Female postpartum euthanasia (21 days) was conducted for each generation ( $n=6 /$ group) to collect liver samples. Real-Time quantitative PCR was performed to evaluate the peroxisome proliferator-activated receptor alpha $(\mathrm{PPAR} \alpha)$, retinoid $\mathrm{X}$ receptor alpha $(\mathrm{RXR} \alpha)$, liver $\mathrm{X}$ receptor alpha $(\mathrm{LXR} \alpha)$, sterol regulatory element binding protein 1c (SREBP-1c). ACT $\beta$, G3PDH and 18S rRNA were used as internal controls. The fatty acid profile of the hepatic tissue was evaluated by the adapted Bligh Dyer Method.

Results: The hepatic omega-3 profile was 10 folds higher for the OM group than the OM-CTL $(\mathrm{P}<0.001)$ and 15 folds than the CTL $(\mathrm{P}<0.001)$. An upregulation was observed in LXR $\alpha$ expression across generations $(\mathrm{P}<0.001)$. F1 had higher expression of RXR $\alpha$ than F2 $(P<0.001)$, however, it was not different from G0. PPAR $\alpha$ expression was not affected by OM treatment, although a reduction was observed in the CTL group from $\mathrm{G} 0$ to $\mathrm{F} 2(\mathrm{P}=0.03)$. SREBP-1c expression was higher in the CTL-CTL than the OM-OM group for $\mathrm{F} 1$ generation $(\mathrm{P}=0.04)$, and an upregulation was observed for all groups from $\mathrm{F} 1$ to $\mathrm{F} 2(\mathrm{P}<0.001)$.

Conclusions: PUFAs affected the expression of transcription factors related to lipolysis and lipogenesis, and this effect seems to be cumulative throughout generations. 
P036

\section{The Omega-3/Omega-6 Ratio in the Regulation of Lipogenesis in the Prepartum Period of Successive Generations in Rats}

\author{
Carolina Bespalhok Jacometo, Diego A.V. Acosta, \\ Patrícia Mattei, Eduardo Schmitt, Francisco A.B. Del Pino, \\ Marcio Nunes Corrêa \\ Universidade Federal de Pelotas
}

Background: Besides the polyunsaturated fatty acids effect on gene expression regulation, the omega-3/omega- 6 ratio could influence the relationship between lipogenic and lipolytic effects.

Objectives: To evaluate the expression of a transcription factor and a target gene related to lipid metabolism by the omega-3/omega- 6 ratio in the prepartum period through consecutive generations.

Methods: Eighteen female Rattus norvegicus - Wistar/UFPel, 8 weeks old, were separated in two groups: 1) diet rich in omega-6, with soybean oil (CTL), 0.4/1 ratio, and, 2) diet rich in omega-3, with flaxseed oil (OM), 13.6/1 ratio. The diets were elaborated in accordance with AIN-93 recommendations. After $30 \mathrm{~d}$ of adaptation the rats were mated. The males received only the control diet. The F1 females were selected at weaning $(n=16 /$ group $)$ and divided into three groups: females from OM group that continued receiving the same diet (OM-OM), females from OM group that began to receive diet containing soybean oil (OM-CTL), and females from CTL group that continued to receive the same diet (CTL-CTL). These animals were mated at $60 \mathrm{~d}$ old as described (G0). The F2 ( $\mathrm{n}=16$ /group) was selected as F1 and the same groups were maintained: OM-OM-OM, OM-CTL-CTL, and CTL-CTL-CTL. The F2 was mated as F1. Female prepartum euthanasia (19 \pm 1 days of pregnancy) was conducted for each generation ( $n=4 /$ group) to collect liver samples. Real-Time quantitative PCR was performed to evaluate the sterol regulatory element binding protein 1c (SREBP-1c), fatty acid synthase (FAS) and acetyl-CoA carboxylase $\alpha$ (ACACA). ACT $\beta$, G3PDH and 18S rRNA were used as internal controls.

Results: An upregulation across generations was observed in SREBP-1c (G0-F2, P < 0.01, F1-F2, P = 0.01) and in FAS (G0-F2 and $\mathrm{F} 1-\mathrm{F} 2, \mathrm{P}<0.01)$, with no differences between groups. A contrasting behavior was observed for ACACA expression, with differences between groups in the F1, a higher expression in OM-OM, an intermediated level for OM-CTL and a lower for CTL-CTL $(\mathrm{P}<0.01$ for all pairs) and along generations, the $\mathrm{OM}$ increased from $\mathrm{G} 0$ to $\mathrm{F} 1(\mathrm{P}<$ $0.01)$ while CTL decreased ( $\mathrm{P}<0.01)$.

Conclusions: Both omega3/omega6 ratio affected the expression of transcription factors related to lipogenesis, and this effect seems to be cumulative throughout generations.

\section{P037 \\ Nutritional Status of Children and Adolescents in Ribeirão Preto, Sao Paulo, Brazil}

Carolina de Almeida Coelho, Jacqueline Pontes Monteiro

Departamento de Puericultura e Pediatria HCFMRPUSP

Introduction: Despite the increasing rate of overweight and obesity in children and adolescents in a lot of cities in Brazil, nutritional deficiencies are still a concern. Even paradoxical, micronutrients deficiencies are associated with overweight and obesity.

Aim: To determine the nutritional status and analise the body composition of a sample of children and adolescents in Ribeirão Preto, Sao Paulo, Brazil and compare data collected in public and private schools. In a second study these same individuals will be analyzed in the way they respond to a set of supplementation of vitamins through proteomics analysis.

Methodology: During April to June of 2012 individuals of 9-13 years were selected from two public schools and one private school in the city of Ribeirão Preto. We measured weight, height and body composition. BMI was calculated using the formula weight $/$ height $^{2}$. We used a digital scale (Plenna), stadiometer (Alturexata) and bioelectrical impedance device (TBW Biodynamic 450). To classify the nutritional status were used charts BMI/age of WHO (2007) for 5-19 years for each sex. To assess the adequacy of the percentage of body fat were used reference for individuals of 7-17 years: 10.01 to $20 \%$ for boys and 15.01 to $25 \%$ for girls (Bristish Journal of Nutrition, v.63, n.2, 1990).

Results: We evaluated 166 boys and 168 girls. The overall prevalence of underweight, normal weight, overweight and obesity was $5.3 \%, 54.2 \%, 24.4 \%$ and $16.1 \%$, respectively, in girls and $7.8 \%$, $50.6 \%, 223 \%$ and $19.3 \%$, respectively, in boys. Excess body fat was found in $91 \%$ of girls and $92.1 \%$ of boys. In private school the prevalence of underweight, normal weight, overweight and obesity was $3.8 \%, 54.7 \%, 30.2 \%$ and $11.3 \%$, respectively, in girls and $0 \%, 42.8 \%$, $26.2 \%$ and $31 \%$ in boys, while the prevalence of excess body fat was $90.6 \%$ for girls and $92.8 \%$ in boys. In public schools, the prevalence of underweight, normal weight, overweight and obesity was $6.1 \%$, $53.9 \%, 21.7 \%$ and $18.3 \%$, respectively, and $10.5 \%$ girls, $53,2 \%, 21 \%$ and $15.3 \%$, respectively, in boys, and that excess body fat was $91.3 \%$ in girls and $91.9 \%$ in boys.

Conclusions: The prevalence of overweight in individuals of 9-13 years in the city of Ribeirão Preto-SP is high, and there is no difference between the prevalence of excess body fat in the private and public schools, in both sexes. From this information it will be possible to investigate the possible association between excess weight and nutritional deficiencies.

Financial support: FAPESP (2012/00783-2). 


\section{P038}

\section{Evidence of Weight Regain after 36 Months of Roux En Y Gastric Bypass}

\author{
Carolina Ferreira Nicoletti, Carolina Hunger Malek-Zadeh, \\ Wilson Salgado-Filho, Julio Sergio Marchini, \\ Carla Barbosa Nonino \\ Faculty of Medicine of Ribeirao Preto
}

Background: Obesity is a complex disease and involves genetic, behavioral and environmental factors. Studies have shown a 30 to $70 \%$ genetic influence on body mass. Research allowed the identification of single nucleotide polymorphisms (SNPs) that may influence individual responses to food. The number of bariatric surgeries performed has greatly increased in recent decades and weight loss maintenance is difficult to achieve for some patients that have regained weight in the late postoperative period. Also, regardless of the strategy used in the control/treatment of obesity, the individual response to interventions is highly variable.

Objectives: Analyze evidence of weight regain after 36 months of roux-en y gastric bypass.

Methods: Anthropometric data were analyzed (weight, height and Body Mass Index - BMI) in the preoperative and postoperative period $(12,24$ and 36 months after surgery) in 150 patients undergoing Roux en $Y$ gastric bypass.

Preliminary Results: $95.5 \%$ of patients had grade III obesity before surgical treatment. It was observed weight loss of $47.2 \pm 13.6$ $\mathrm{kg}(30.7 \%)$ at the end of 12 months and $6.4 \pm 9.3 \mathrm{~kg}(7.8 \%)$ from 12 to 24 months. After 36 months it was observed that $51.7 \%$ of the patients had weight regain: $32 \%$ regained more than $10 \%$ of lost weight $(18.3 \pm 9.3 \%)$ and $68 \%$ had regained from 0.2 to $9.9 \%$.

Conclusions: Bariatric surgery promotes important weight change in the postoperative period, as evidenced by the great weight loss after 12 months of surgery. However, it is observed that a number of patients recover weight in the late postoperative period. Based on the findings of weight evolution, the second step of this study is to detect the SNPs in obese subjects submitted to GRYB and their association with the different responses to the surgical procedure during the late postoperative period (36 months). This study will identify some SNPs (Trp64Arg in gene ADRB3, - Pro12Ala in gene PPARG2, $-3826 \mathrm{~A} / \mathrm{G}$ in gene UCP1, $-866 \mathrm{G} / \mathrm{A}$ and Ala55Val in gene UCP2, $11482 \mathrm{G}>\mathrm{A}$ in gene PLIN4, $-393 \mathrm{~T}>\mathrm{C}$ and C825T in the protein $\mathrm{G}$ gene, GNAS1and GNB3 subunits) that determine weight changes after GRYB. Genotype analysis will be performed by the method of allele discrimination using the real-time polymerase chain reaction (PCR).

Financial support: FAPESP (2012/05539-2).

\section{P039 \\ Therapeutic Role of Acetate-Producing Probiotic on Allergic Airway Inflammation}

\author{
Caroline M. Ferreira, Eduardo Mendes, \\ Fernanda Yamamoto Silva, Niels O. Câmara, \\ Mauro Martins Teixeira, Wothan Tavares de Lima, Rui Curi \\ Universidade de São Paulo
}

Background: Asthma is a chronic inflammatory lung disease that affects about 300 million people worldwide. Genetic and environmental factors have been associated with triggering the disease. An important envirommental factors, it is the exposure to microbes in early life that protects against allergies, called as hygiene hypothesis. Since this hypothesis was proposed, many studies have investigated the role of intestinal microbiota on the modulation of the immune system and allergy. Additionally, studies have shown differences in the composition of the microflora between healthy and allergic infants. Alongside scientific findings about gastrointestinal micorbiota influence on allergy, probiotic market is expanding with promises for the treatment and prevention of disease. However, it is unclear the role for probiotics in treating allergic disorders. Evidences in the literature support the possibility that short chain fatty acids (SCFA) produced by some intestinal bacteria, as acetate, it could be a mechanism involved in the immune system modulation by intestinal microbiota. The hypothesis of the present study is that probiotic bacteria could modulate allergic airway inflammation via production of SCFA, such as acetate.

Methods: We investigated the role of two different probiotics, $B$. longum and $B$. adolescentis on airway inflammation. The choice of two probiotics was based on the fact that $B$. longum is associated with allergy protection and great acetate production, and B. adolescentis is not associated with allergy protection and it is not a relevant acetate producer. We also investigated wether host genetics may influence on the probiotic effects.

Results: We found that while $B$. longum reduced eosinophils cells, airway hyperresposiveness and mucus production in Balb/c and C57BL/6 mice. Surprisely, B. adolescentis treatment also decreased airway inflammation in Balb/c mice but did not change mucus production. Also, B. adolescentis did not affect Th2 mediated airway inflammation in C57BL/6 mice.

Conclusions: In conclusion, our data show that B. longum can affect Th2 mediated airway inflammation independent of host genetic. However, B. adolescentis changes some characteristics of airway inflammation in Balb/c but did not change in C57BL/6. Thus, it is possible that great acetate production by B. longum may be an important key to modulate all factors involved on Th2 mediated airway inflammation, independently of host genetics variation.

Financial support: CAPES. 
P040

\section{lodide Excess Reduces the Activity of Sodium-lodide Symporter (NIS) Promoter: the Role of PAX8 and P65 Transcription Factors on the Inhibitory Effects of This Oligoelement}

Caroline Serrano-Nascimento, Juan Pablo Nicola,

Universidade de São Paulo/Universidad de Córdoba

Background: Iodide is an essential micronutrient for thyroid hormone (TH) synthesis and an important regulator of thyroid function. Iodide is transported across the basolateral membrane of thyrocytes by NIS, which is the limiting step for TH synthesis. However, iodide excess rapidly reduces NIS mRNA poly(A) tail length and consequently its half-life and expression, mechanisms that take part in the autoregulation of thyroid function. In longer periods of treatment (12-24h) the reduction of NIS expression persists, even though there is no alteration in the transcript poly(A) tail length. This indicates that mechanisms other than posttranscriptional are involved on NIS expression regulation by iodide. NIS gene expression is upregulated mainly by two transcriptional factors, PAX8 and p65. Thus, this study aimed to investigate if these transcriptional factors are involved on the negative effects of iodide excess on NIS gene expression.

Methods: Rat thyroid cells ( $\mathrm{PCCl} 3$ cells) were transfected with plasmids containing NIS full promoter region, its enhancer, or NIS promoter region with mutations on PAX8 or p65 binding sites. Cells were treated 12 and $24 \mathrm{~h}$ with $\mathrm{NaI}\left(10^{-3} \mathrm{M}\right)$. NIS promoter activity was evaluated by luciferase assay. Imunocytochemistry and fractionation of nuclear and cytoplasmic proteins were also performed to identify the cellular localization of these transcriptional factors. ChIP assay was done to investigate the binding of these factors to their specific regions on NIS promoter.

Results: NIS promoter activity was reduced 12 and $24 \mathrm{~h}$ after iodide treatment. This effect was abrogated in cells transfected with both NIS mutated promoters. Furthermore, iodide reduced the amount of PAX8 and p65 in the nucleus, and increased their content in the cytoplasm. It was also observed a reduction in the association of these factors to their specific binding regions on NIS promoter.

Conclusions: The results presented herein demonstrated that transcriptional mechanisms are involved in the iodide negative effects on NIS expression, when cells are exposed to iodide for longer periods of time. The reduced PAX8/p65 nuclear content and binding to NIS promoter seem to underlie these effects. These data indicated that multiple mechanisms are part of the iodide autoregulatory effect on thyroid, which guarantees its uptake for proper TH synthesis. Moreover, this mechanism also prevents the harmful effects that could be triggered by iodide excess on thyroid.

Financial support: FAPESP (2009/50175-6).

\section{P041}

Effect of Lycopene on Oxidative Damage Induced by Hyperglycemia in Human Erythrocytes in Vitro

Caroline Silveira Martinez, Hemerson Silva Rosa, Márcia Rósula Poetini, Ana Ceolin Colpo,

Maria Eduarda Lima, Felipa Melgarecho Bassante, Julio Cesar Mendes Soares

Federal University of Pampa

Background: The chronic hyperglycemia determines a series of biochemical phenomena, which are involved in the genesis of the oxidative stress in diabetes mellitus. Lycopene is a dietary carotenoid synthesized by plants and microorganisms. The antioxidant activity of lycopene has been extensively evaluated based on its ability to scavenge free radicals in cell culture and in animal models.

Objectives: The aim of this study was to evaluate the effects of the Lycopene on the oxidative damage induced by different fructose concentrations in human erythrocytes in vitro.

Methods: Samples of human red blood cells, donated from the hospital of Itaqui (RS, Brazil) were used. The erythrocytes were incubated at $37^{\circ} \mathrm{C}$ for 24 hours with the following concentrations of fructose 5, 10, 20, 30 and $50 \mathrm{mM}$ with or without lycopene. Lycopene (10\% powder, Deg Chemical Products Importation LTDA, São Paulo, Brazil) was added into erythrocytes in increasing concentrations $(0.01,0.02$ and $0.03 \mathrm{mM})$. After incubation, the lipid peroxidation was determined according to the method of Thiobarbituric Acid Reactive Substances (TBARS) formation. Data were analyzed by Analysis of Variance (ANOVA) followed by a Tukey's test. $p$ values $\leq 0.05$ were considered as significant.

Results: The TBARS assay showed that with increasing concentrations of fructose, the lipid peroxidation also increased, reaching a peak at $50 \mathrm{mM}$ fructose. We observed that all concentrations of lycopene tested demonstrated a significant reduction of TBARS in any fructose concentration evaluated, so there were no differences between concentrations of lycopene. Therefore, it was found that the lowest lycopene concentration has the same effect of the highest concentration tested being able to reduce until $60 \%$ of TBARS.

Conclusions: The results showed a dose-dependent effect of the concentration of fructose and TBARS production, and that all concentrations of lycopene were able to significantly reduce oxidative damage caused by hyperglycemia in human erythrocyte like tested in vitro. 


\section{P042}

\section{Effects of Milk Oligosaccharides During Pregnancy on Mice Mono-Associated with Bifidobacterium Bifidum}

\author{
Caroline Thum, Kikuji Itoh, Adrian L. Cookson, \\ Wayne Young, Don E. Otter, Warren C. McNabb, \\ Alison J. Hodgkinson, Jolon Dyer, Nicole C. Roy \\ Agresearch Grasslands
}

Epidemiological studies over the past 20 years have shown how changes in maternal food intake during fetal and early postnatal development affect the offspring's susceptibility to metabolic diseases later in life. Recently, changes in maternal intestinal microbiota by supplementation with probiotics and prebiotics have been reported to beneficially impact the development, maturation and microbial colonisation of the neonatal gastrointestinal tract (GIT). Among ruminant milk, goat milk has the oligosaccharides profile and structure most similar to human milk. These oligosaccharides act as prebiotics by increasing the population of health promoting bacteria such as bifidobacteria. Research done in our laboratory showed that bifidobacterial strains (Bifidobacterium bifidum, B. longum ssp longum and $B$. breve) isolated from baby faecal samples were able to ferment goat milk oligosaccharides (GO) purified (35\%) from goat whey. Of the isolated strains, B. bifidum (AGR 2166) was the best at fermenting purified GO based on growth and short chain fatty acids profiles. The objective of this study was to determine if dietary GO change physiological characteristics of the large bowel in pregnant mice monoassociated with the $B$. bifidum. A suspension of $B$. bifidum $\left(10^{7} \mathrm{CFU}\right)$ or a sterile phosphate buffer saline control was orally inoculated into germ-free mice fed either with GO or control diet throughout mating and pregnancy. B. bifidum concentration in faeces remains stable throughout the pregnancy of colonised groups. Mice were euthanised 1 to 3 days before delivery. No differences in maternal spleen, liver, and mesenteric lymph nodes weight were found among treatments. There were no changes in colonic crypt length and goblet cell numbers in transverse sections of the colon for any of the treatments. The combination of GO feeding and B. bifidum inoculation (compared to $B$. bifidum inoculation alone) increased weight and length of the maternal GIT. Future work will include the analysis of maternal colon and foetal gene and protein expression profiles from these groups. Together, these analyses will provide insights into how maternal supplementation with GO and B. bifidum colonisation may improve the intestinal development and colonisation of the newborn.

Financial support: Agresearch Grasslands.

\section{P043}

\section{Folic Acid Ingestion and Risk of Experimental Colorectal Carcinogenesis in Rats}

\author{
Celia Cohen, João Felipe Rito Cardoso, \\ Fernanda Aparecida Domenici, Raquel Alves Dos Santos, \\ Mônica Silva De Souza Meirelles, Sérgio Britto Garcia, \\ Helio Vannucchi
}

School of Medicine of Ribeirão Preto, USP

Background: Recently the role of folic acid food fortification in colorectal cancer has been discussed. Data from experimental studies suggested that folic acid may have a dual modulating role in colorectal cancer development and progression, depending on time and dose.

Objectives: This study aimed to evaluate the effects of folic acid fortification and supplementation followed by fortification in colorectal carcinogenesis in rats.

Matherials and Methods: Wistar rats received either Standard AIN-93M diet during all experiment (G1), diet without folic acid during all experiment (G2), diet with $3.25 \mathrm{mg}$ of folic acid during all experiment (G3) or diet with $5 \mathrm{mg}$ of folic acid before carcinogenesis induction followed by a diet with $3.25 \mathrm{mg}$ of folic acid through the experimental period (G4). Colorectal carcinogenesis was induced by 4 doses of MNNG $(5 \mathrm{mg} / \mathrm{mL})$ twice a week during 2 weeks. Analysis: serum folic acid and plasma homocysteine, colonic S-adenosylmetionine (SAM) and S-adenosylhomocysteine (SAH), caspase-3 and DNA damage by Comet Assay. Statistical analysis: ANOVA, $\mathrm{p}<0.05$.

Results: Both supplemented groups had increased serum concentration of folic acid $(p<0.05)$. G2 presented a reduced concentration of this vitamin and an increased level of homocysteine $(\mathrm{p}<0.05)$. G3 had higher SAM than the other groups $(p<0.05)$. G2 showed an increased SAH when compared to the other groups $(p<0.001)$ and $\mathrm{G} 3$ had lower SAH than G4 ( $<<0.01)$. The comet assay showed that group G3 had lower DNA basal damage, followed by G2 and G4 (p < $0.05)$. G1exhibited higher DNA basal damage than the other groups studied $(\mathrm{p}<0.05)$.

Conclusions: Folic acid fortification (G3) does not seem to increase colorectal carcinogenesis risk in rats. Moreover, fortification with folic acid after supplementation with the higher dose (G4) increases DNA basal damage and SAH colonic levels and may be hazardous to chemical colorectal carcinogenesis.

Financial support: FAPESP (2010/50164-1). 
P044

Effects of Strength Training Associated with Fish Oil Supplementation on Lymphocyte Intracellular Signaling

Cesar Miguel Momesso, Gustavo Barquilha,

Adriano Santos, Rui Curi, Tania Pithon-Curi,

Sandro Hirabara, Renata Gorjão

UNICSUL - ICAFE

Background: High intensity strength exercise may induce an inflammatory response that can alter lymphocyte activation mechanisms (RAMEL, 2003). Studies have shown that n-3 fatty acid supplementation reduces inflammatory process in subjects submitted to aerobic exercise (PEDERSEN, 2000). However, there are no studies related to strength exercise.

Objectives: Evaluate the effects of strength exercise training and n-3 fatty acid supplementation on lymphocyte activation mechanisms.

Methods: Sixteen untrained male subjects ( $25 \pm 5$ years of age) were evaluated in this study. The strength training protocol was performed during seven weeks, three times a week alternation between hypertrophy and strength sessions. Eight subjects were supplemented for 2 months with $3 \mathrm{~g}$ /day of fish oil (FO) (54\% EPA and 23\% DHA). Blood was taken from trained subjects before and immediately after the first session of hypertrophy exercise and after 8 weeks of training and supplementation. CD25 expression in $\mathrm{CD} 4{ }^{+}$lymphocytes, DNA fragmentation and membrane integrity were evaluated by flow cytometry, proliferative capacity were determinated by incorporation of $\left[2-{ }^{14} \mathrm{C}\right]$-thymidine. Akt, ERK1/2 and PKC-Zeta phosphorylation were detected by western-blotting.

Results: The proliferative capacity of lymphocytes decreased after the first hypertrophy session (4362 \pm 370 to $2630 \pm 304 \mathrm{cpm})$. After two months of training no difference was observed. The supplementation did not alter proliferation. There were no differences in DNA fragmentation and membrane integrity in any situation. Percentage of CD25 positive cells was decreased after the first hypertrophy session ( $22 \%$ to $9 \%$ of total lymphocytes) but returned after 8 weeks of training. Akt and ERK1/2 (proteins related to lymphocyte proliferation) phosphorylation were decreased after the first hypertrophy session, however, no effect was observed in the end of the training. Likewise, fish oil supplementation had no effect. Discussion: The present study indicates that there is a reduction in lymphocyte function after a week of high intensity session of hypertrophy exercise in untrained individuals. This is due to an inhibition of intracellular pathways related to proliferation. Fish oil did not alter lymphocyte response.

Conclusions: The n-3 fatty acid supplementation associated with strength training did not alter lymphocyte signaling pathways. However, other studies showed that isolated n-3 fatty acids modulate lymphocyte signaling pathways.

Financial support: FAPESP, CAPES, CNPQ.

\section{P045 \\ Dietary Combination of Conjugated Linoleic Acid and Phytosterols Modulates the Body Composition in Lean and Obese Sprague- Dawley Rats}

\author{
Rafaela da Silva Marineli, Cibele Priscila Busch Furlan, \\ Anne Y. Castro Marques, Camilla Bertuzzo Veiga, \\ Mário Roberto Maróstica Júnior \\ UNICAMP
}

Conjugated linoleic acid (CLA) and phytosterols have been studied for their beneficial effects on health in animal models and clinical studies. Modulation of body composition has been attributed to these lipid compounds with contradictory findings. We investigated the effect of CLA and/or phytosterols supplementation on food intake, body weight gain and body composition in lean and obese rats. Seventy-five Sprague-Dawley rats were fed with high-fat diet $(35 \%)$ or normal-fat diet $(9 \%)$ supplemented with $2 \%$ of each lipid compound. Animals were divided into nine groups: Standard group (P), Control high-fat group (HF), Control linoleic acid group (LA), three high-fat groups fed diets added CLA (HC), phytosterol (HP) and CLA plus phytosterols $(\mathrm{HC}+\mathrm{P})$, three normal-fat goups fed diets added CLA (C), phytosterol (P) and CLA plus phytosterols $(\mathrm{C}+\mathrm{P})$ for 9 weeks. The weight gain was monitored weekly and the food intake every 2 days during the experimental period. Animals were sacrificed by decapitation preceded by 12 -h-fasting. For the assessment of body composition the carcasses were lyophilized and stored at $-20^{\circ} \mathrm{C}$. Body protein was determined by the semi-micro Kjeldahl method and ashes were quanti?ed by incineration in a muf?e furnace. Body total fat was determined by the method of Bligh and Dyer extraction. Daily food intake was statistically lower in CLA groups, $13 \%$ for lean and $10 \%$ for obese rats $(\mathrm{p}<0.05)$. The association between CLA and phytosterols also significantly decreased food intake $(10 \%)$ and body weight gain $(14 \%)$ in obese rats compared to control groups $(\mathrm{p}<$ 0.05 ). Furthermore, the body weight gain was $5 \%$ decreased in $\mathrm{C}$ group compared to control group in lean rats. In relation to body composition, phytosterols supplementation significantly increased the lean mass $(30 \%)$ and decreased the body fat $(19 \%)$ in obese rats $(\mathrm{p}<$ $0.05)$. In addition, the supplementation with CLA, phytosterols and their mixtures decreased the body fat, $14 \%, 15 \%$ and $13 \%$, respectively, in lean rats. There was no significant difference among the experimental groups for ash content for both obese and lean rats. The supplementation with CLA and/or phytosterols showed an important effect in reduce food intake, body weight gain and body fat leading to an improvement in the prevention and treatment of obesity. In summary these finds suggest that the dietary association between CLA and phytosterols could be beneficial for the prevention of metabolic syndrome by suppressing fat accumulation.

Financial support: $\mathrm{CNPQ} / \mathrm{FAPESP}$. 


\section{P046}

Plasma Leptin and Insulin Concentrations Are Decreased in Obesity Rats Fed with High-Fat Diet When Added Conjugated Linoleic Acid and/or Phytosterol

\section{Cibele Priscila Busch Furlan, Rafaela Silva Marineli, Anne Castro Marques, Mário Roberto Maróstica Júnior \\ School of Food Engineering, University of Campinas}

Conjugated linoleic acid (CLA) has several benefits and an important role in inflammation and body alterations principally in adipose tissue, in obesity. Phytosterols also have been shown effects in reduce the formation of atherosclerosis and inflammation. This study aimed to determine the effect of CLA and/or phytosterols on leptin and insulin of Sprague-Dawley rats fed with high-fat diet. Twenty-one-daysold Sprague-Dawley rats were divided into five groups $(n=5)$ : standard group (P), standard High-Fat group (HF) and three high-fat groups fed diets added CLA (HC), phytosterol (HP) and phytosterols plus CLA (HS). The experimental period lasted 65 days. Analyses of leptin and insulin were performed using enzyme-linked immunosorbent assay (ELISA) method. One-way ANOVA with the Tukey test for post-hoc analyses was applied to evaluate statistical differences between groups. All results were compared to HF group. Leptin decreased $23 \%$ in HC, $4 \%$ in HP and $10 \%$ in HS groups ( $\mathrm{p}<0.0001$ ). Insulin, among all the high fat groups, did not presented statistically significant difference, but decreased $7 \%$ in HC, $15 \%$ in HP and $19 \%$ in HS groups $(\mathrm{p}<0.05)$. Probably, these diminishments of the leptin and insulin have been associate the reduction inflammatory condition in the obesity proportionate by phytosterols and CLA, as well as to reduction the fat tissue in rats fed CLA, as vastly reported in literature. All groups supplemented the CLA and/or phytosterols showed an important effect in reduce leptin and insulin leading to an improvement in the obesity.

Financial support: FAPESP, CNPQ.

\section{P047 \\ Supplementation with Conjugated Linoleic Acid and/or Phytosterol Reduced Organs and Body Weight of Diet-Induced Obese Sprague-Dawley Rats}

\section{Cibele Priscila Busch Furlan, Rafaela Silva Marineli, Mário Roberto Maróstica Júnior}

\section{Universidade Estadual de Campinas}

CLA and phytosterols are recognized to reduce body weight. This study investigated the effect of CLA and/or phytosterols on organs and body weight of rats fed with high-fat diet. Twenty-one-days-old Sprague-Dawley rats were divided into five groups $(n=5)$ : standard group (P), standard High-Fat group (HF) and three high-fat groups fed diets added CLA (HC), phytosterol (HP) and phytosterols plus CLA (HS). The experimental period lasted 65 days. During the experiment the rats was weighed three times a week. After sacrificed the organs were removed and weighed. All results were compared to HF group. Supplemented groups HC, HP and HS showed a decrease on body weight of $6 \%, 13.5 \%$ and $17 \%$ respectively $(p=.0001)$. Spleen, kidney, pancreas, femur, heart and adiposity-tissue decreased significantly in all supplemented groups $(\mathrm{p}<0.05)$. In average heart and spleen decreased $7 \%$, pancreas $13 \%$, kidney $11 \%$ and femur $17 \%$. The epididymal fat content decreased $25 \%$ in $\mathrm{HC}, 43 \%$ in $\mathrm{HP}$, and $45 \%$ in HS group and retroperitoneal fat decreased $52 \%$ in $\mathrm{HC}, 40 \%$ in HP and $53.5 \%$ in HS group $(\mathrm{p}<0.0001)$. The intestine weight increased $8 \%$ in HP and HS and decreased $0.5 \%$ in HC $(\mathrm{p}<0.05)$. Probably, these reductions on organs weight could be due to modulation peroxisome-proliferator-activated-receptors-gamma in groups supplemented with CLA, and inhibition of the ACAT activity and competition for cholesterol transport and esterase activity promoted by phytosterols, which are reported in the literature. CLA and phytosterol have contributed to the reduction of body weight and affected the weight of organs, not only weight the adipose tissue.

Financial support: FAPESP/CNPQ.

\section{P048 \\ Obesity: The Role of Free Fatty Acids in Subclinical Inflammation}

Crislaine Graças Almeida, Josefina Bressan

Universidade Federal de Viçosa

Obesity and metabolic disorders are associated with inflammation, that is characterized by high levels of pro-inflammatory cytokines such as tumor necrosis factor-alpha (TNF- $\alpha$ ), interleukins 6 and $1 \beta$ (IL-6 and IL-1 $\beta$ ), and macrophage infiltration in the adipose tissue. The activation of that inflammation occurs by increased expression of nuclear factor kappa $\mathrm{B}(\mathrm{NF \kappa B})$ and activation of c-Jun $\mathrm{N}$-terminal kinase (JNK), with participation of toll like receptors (TLR). Therefore, in order to study the role of different fatty acids in the activation of these mechanisms, we identified publications from searches on electronic data-bases, using the following keywords: "inflammation", and/or "TLR", "high-fat diet", "saturated fatty acid", "unsaturated fatty acid", "arachidonic acid", "obesity". The titles and abstracts of all identified studies by the electronic searches were screened, overall, 15 studies in vivo $(n=8)$ e in vitro $(n=7)$ were included. According to the studies found, the long chain saturated fatty acids promote adipocyte hypertrophy and stimulate pro-inflammatory genes such as IL-1 $\beta$, IL- 6 and IL- 8 , TNF- $\alpha$, monocyte chemotactic protein-1 (MCP-1) and matrix metallopeptidase 9 (MMP-9), it also increases resistin secretion, serum amyloid A 1 (SAA1), the production of reactive oxygen species (ROS) and the activity of JNK and $\mathrm{NF} \kappa \mathrm{B}$, besides, reduces the expression of IL-10. The monounsaturated fatty acids are associated with adypocyte hypertrophy and with the increase of the triglyceride content. Polyunsaturated n-3 fatty acids (n-3 PUFAs), however, exerts anti-inflammatory effects, reducing the expression of sTNF-R1 and sTNF-R2 (soluble tumor necrosis factor receptors 1 and 2), MCP-1, SAA3, suppressor of cytokine signaling 3 (SOCS-3) and the synthesis of hyaluronic acid, and increasing secretion of adiponectin. These effects of dietary n-3 PUFAs are dependent on the intake of n-6 PUFAs, which suggests that at low levels of n-3 fatty acid intake, n- 6 fatty acids are associated with high levels of inflammatory markers. Furthermore, linoleic acid, a n-6 
essential fatty acid, leads to production of arachidonic acid, which is the substrate for proinflammatory and prothrombotic eicosanoids. The high adipose tissue arachidonic acid content is associated with an increasing risk of the metabolic syndrome. We conclude that the control of the lipid profile of the diet is a powerful strategy to control inflammation and metabolic disorders.

\section{P049 \\ Influences of Circus Physical Activity Program on Cardiorespiratory Fitness $\left(\mathrm{VO}_{\mathbf{2}} \mathbf{m a x}\right)$ in Overweight Children}

\author{
Cristina Neves Borges-Silva, Cristiane Cassoni Goncalves- \\ Santo, Silvia Helena Guirado-Rodrigu, \\ Cesar Miguel Momesso Santos, Renata Gorjao, \\ Tania Cristina Pithon-Curi, Thelma Hoehne Peres Polato \\ University Cruzeiro do Sul
}

Background: Obesity and sedentary lifestyles have a negative impact on cardiorespiratory function, flexibility, strength, resistance and body fat in children and adolescents. Programs that are designed to maintain high childhood physical activity levels may improve physical fitness to mitigate the adverse effects of obesity.

Objectives: The influence of circus physical activity program on cardiorespiratory fitness in normal weight and overweight children were investigated.

Patients and Methods: This study involving sixty girls, 8 to 11 years old, participated in this study. The pubertal girls were divided into four groups: 1) Normal Weight, Control $\left(\mathrm{NW}_{\mathrm{CON}}\right)$, which included 18 girls partaking in no structured physical activity, 2) Overweight, Control $\left(\mathrm{OW}_{\mathrm{CON}}\right)$, which included 15 girls partaking in no structured physical activity, 3) Normal Weight, Physical Exercise $\left(\mathrm{NW}_{\mathrm{EX}}\right)$, which included 15 normal weight girls partaking in circus activity (two days a week in sessions lasting for 60 minutes for 2 years) and 4) Overweight, Physical Exercise $\left(\mathrm{OW}_{\mathrm{EX}}\right)$, which included 12 overweight girls partaking in circus activity. The cardiorespiratory fitness assessment was determined using a maximal multistage $20 \mathrm{~m}$ shuttle run test. To assess the intensity of the circus physical activity, each child wore a heart rate (HR) monitor Polar during the 12-week training period. The following values were determined: a) average of the resting HR each week (HR rest), in beats per minute (bpm), measured during the initial rest interval of 60 seconds, b) average HR during class each week (HR during class), in bpm, measured during the circus class, c) average HR after one minute in the recovery period each week (HR after $1 \mathrm{~min}$ ), in bpm, measured one minute after the end of class and d) variations of HR from one week to the next $(\Delta=$ $H R$ ), in bpm, calculated for three situations (rest, during class and after one min). The data were analyzed by a two-way analysis of variance (ANOVA).

Results: The $\mathrm{OW}_{\mathrm{CON}}$ did not show any differences in the $\mathrm{VO} 2$ max than the $\mathrm{NW}_{\mathrm{CON}}$. The $\mathrm{OW}_{\mathrm{EX}}$ showed less $\mathrm{VO}_{2} \max$ than the $\mathrm{NW}_{\mathrm{EX}}$. The relative gain in performance was greater for the $\mathrm{NW}_{\mathrm{EX}}$ $\left(16 \%\right.$ in $\left.\mathrm{VO}_{2} \max \right)$ than for the $\mathrm{OW}_{\mathrm{EX}}\left(6.8 \%\right.$ in $\left.\mathrm{VO}_{2} \mathrm{max}\right)$. The $\mathrm{OW}_{\mathrm{EX}}$ had a lower variation of heart rate $(\Delta \mathrm{HR})$ at rest and during the sessions of the circus physical activity program than the $\mathrm{NW}_{\mathrm{EX}}$.
Conclusions: This study, being overweight did not affect the functional aspects but did diminish the benefits from physical exercise.

\section{P050}

The Influence of Circus Physical Program on Insulin Sensitivity and Biochemical Parameters in Overweight Children

\author{
Cristina Neves Borges-Silva, Silvia Helena Guirado- \\ Rodrigu, Cristiane Cassoni Gonçalves-Santo, \\ Cesar Miguel Momesso Santos, \\ Thelma Hoehne Peres Polato, Renata Gorjao, \\ Tania Cristina Pithon-Curi \\ University Cruzeiro Do Sul
}

Background: An association between obesity and insulin resistance has been reported in the young, as has the link between physical exercise and improves insulin sensitivity. However, the role of the circus physical program of insulin resistance and obesity in adolescents is less clearly defined.

Objectives: The goal of this study was to investigate the influence of a circus physical activity program in the response of insulin sensitivity and biochemical in normal and overweight children. Patients and Methods: Sixty girls, 8 to 11 years old, participated in this study. The pubertal girls (Tanner stage II to IV) were divided into four groups: 1) Normal Weight, Control $\left(\mathrm{NW}_{\mathrm{CON}}\right)$, which included 18 girls partaking in no structured physical activity, 2) Overweight, Control $\left(\mathrm{OW}_{\mathrm{CON}}\right)$, which included 15 girls partaking in no structured physical activity, 3) Normal Weight, Physical Exercise $\left(\mathrm{NW}_{\mathrm{EX}}\right)$, which included 15 normal weight girls partaking in circus activity and 4) Overweight, Physical Exercise $\left(\mathrm{OW}_{\mathrm{EX}}\right)$, which included 12 overweight girls partaking in circus activity. The volunteers practiced the circus activity two days a week, in sessions lasting 60 minutes, during 2 years. An estimate of insulin resistance was determined by HOMA-IR. glycemia, cholesterol, triglycerides and insulin plasma concentration were also determined.

Results: Overweight children presented hyperinsulinemia, increased HOMA-IR and triacylglycerols levels. On the other hand, overweight exercised children presented a reduction of HOMA-IR (30.5\%), glycemia and triglycerides.

Conclusions: This study shows that the overweight condition is linked with metabolic profile alteration. However, the participation in the program of circus activity improved insulin sensitivity. 


\section{P051 \\ Kefir Grains Incorporated in Different Food Matrices Reduce the Levels of Lipid Peroxidation Products in SHR Rats Induced Metabolic Syndrome}

\author{
Damiana Diniz Rosa, Mariana Moura Dias, \\ Sandra Aparecida Reis, Ana Carolina Machado Fonseca, \\ Nathane Pais Siqueira, Lisiane Lopes Conceição, \\ Luciana Nogueira Gontijo, Leticia Linhares Silva, \\ Mayra Eugênio Rodrigues, \\ Maria do Carmo Gouveia Peluzio
}

Universidade Federal de Viçosa

The fermented milk has often been studied for its possible beneficial physiological effects, such as its antimutagenicity, immunopotentiating activity, antitumor activity, prevention of pathogenic infection and antioxidant role. Kefir has antioxidant potential that interacts with a wide range of species directly responsible for oxidative damage. The objective was to evaluate the antioxidant potential of probiotic kefir produced in different food matrix (cow's whole milk and skim milk) in SHR rats with metabolic syndrome induced by monosodium glutamate (MSG). For the preparation of the solution Kefir grains were inoculated at a concentration of $5 \%(\mathrm{w} / \mathrm{v})$ of fresh milk (whole cow's milk and skimmed milk) and cultivated at $26^{\circ} \mathrm{C}$ for 24 h daily. Forty male SHR rats received intradermal injections of MSG (4 $\mathrm{g}$ of body weight $/ \mathrm{g}$ ) during the first 5 days of life. After 90 days, the rats were divided into groups of 10 animals each and received daily by gavage in $1 \mathrm{~mL}$ solution: G1: whole milk, G2: kefir of whole milk, G3: skim milk and G4: kefir of skim milk, for 10 weeks. At the end of the experiment, the enzymes of the endogenous antioxidant system (catalase and superoxide dismutase) and lipid peroxidation products (malondialdehyde and hydroperoxides) were evaluated in liver tissue. The treatments did not differ in weight gain, food consumption and food efficiency ratio $(p>0.05)$. Animals that received kefir of whole milk were not different from those who received whole milk according to the levels of catalase $(p=0.929)$, superoxide dismutase $(p=0.376)$ and hydroperoxides $(p=0.692)$. However, lower levels of malondialdehyde were found in kefir of whole milk group $(\mathrm{p}<0.001)$. When compared with the group that received skim milk, the animals that received kefir of skim milk showed lower levels of lipid hydroperoxides $(\mathrm{p}=0.028)$. Both fermented whole and skim milk were unable to alter levels of the endogenous liver enzymes of the antioxidant system. Nevertheless, lower levels of lipid oxidation products were observed in animals treated with kefir. Therefore, kefir is a useful potential candidate as natural antioxidant supplements in human diet.

Financial support: CNPQ, FAPEMIG.

\section{P052 \\ Effects of the Maternal Intake of Palm Oil, Interesterified Fat or Trans Fat, During Gestation and Lactation, on the Adult Offspring's Hypothalamic Insulin Sensitiveness}

\author{
Daniela de Barros Mucci, Letícia Oliveira Rodrigues, \\ Érica Soares Souza, Kenia Pereira Bispo, \\ Fátima Lúcia Carvalho Sardinha, Kelse Tibau Albuquerque \\ Universidade Federal do Rio De Janeiro
}

Background: Changes in the fatty acid (FA) composition of the diet, during the critical phases of fetal development and neonatal period, may lead to permanent metabolic adaptations, with changes in the homeostasis of different tissues and systems.

Objectives: Evaluate the effects of the maternal intake of different trans fat substitutes, during gestation and lactation, on the adult offspring's hypothalamic insulin sensitiveness.

Methods: Female Wistar rats received isocaloric and normolipidic diet during pregnancy and lactation and their pups were divided in 4 groups, according to the mother's lipid source: palm oil (PG), Interesterified fat (IG), partial hydrogenated (trans group - TG) or soybean oil (control group - CG). A standard diet was given to the post-weaning pups. During gestation and lactation, mothers' weight and diet intake were accessed. The pups' weight was evaluated from birth to weaning. P90 rats received intracerebroventricular (icv) insulin injection, food intake was measured 12 and 24h later and adipose tissues were taken and weighted. Proteins involved in the insulin signaling cascade (IR and Akt) were quantified. ANOVA one-way and Newman Keuls or Student's t tests were used as appropriate. The level of significance was set at $\mathrm{P}<0.05$.

Results: Food intake and weight were not significantly different among groups for both mothers and pups. The changes in the FA maternal diet composition did not interfere with the offsprings' adipose tissues total weight. After icv insulin injection, only CG presented significantly lower food intake, compared to icv saline injection. PG's IR content was $64,1 \%$ and $69,19 \%$ higher, compared to $\mathrm{CG}$ and TG, respectively, $\mathrm{p}<0.05$. Akt quantification was not significantly different. The increase in the IR content observed in the PG was not enough to lower their food intake.

Conclusions: These findings suggest that the different trans fat substitutes used in this study decreased the insulin anorexigenic effect, even though changes in the content of the proteins involved in the early steps of the insulin's transduction cascade were not found.

Financial support: FAPERJ. 


\section{P053 \\ Evaluation of C677T and A1298C \\ Polymorphisms of MTHFR Gene and Its Association with Alcohol Consumption and Smoking in Patients with Head and Neck Tumors}

\author{
Daniela Fernandes Torralbo, Francine Rossetti Delboni, \\ Bianca Bianco, André Nimtz Rodrigues, Kelly Oliveira, \\ Fernando Claret Alcadipa, Monica Vannucci Lipay \\ Universidade Ferderal de São Paulo
}

Background: Among the risk factors related to development of head and neck cancer are alcohol and smoking using, who are responsible for a deficit of micronutrients such as folic acid, vitamin B12, vitamin B6, iron, selenium and zinc. Genetic factors, such as polymorphisms of methylenetetrahydrofolate reductase (MTHFR) gene (C677T and A1298C), involved with folate metabolism, also may be related to the genesis of tumor.

Objectives: Establish the frequency of C677T and A1298C polymorphisms of MTHFR gene in patients with head and neck tumors and in a control group and correlate them to the occurrence of tumors in individuals distinguished with alcohol consumption and their smoking habit.

Methods: We evaluated 55 patients with head and neck tumors with respect to MTHFR genotypes and smoking habits and alcohol consumption, from Outpatient Clinic of the Medicine College in Jundiaí (FMJ) - Sao Paulo, Brazil, as well as 581 healthy individuals selected from the general population without history of associated diseases. Patients with cancer were divided into four groups regarding quantity of alcohol consumed daily (group 1: no consumption, group 2: $1.0-200 \mathrm{~mL}$, group 3: 201.0 to $500.0 \mathrm{~mL}$, group 4: 501.0 to $2000.0 \mathrm{~mL}$ ). Genotyping of polymorphisms was performed by PCRRFLP (C677T) and qPCR (A1298C). For statistical analysis was used chi-square test, with significance level of $p<0.05$.

Results: The number of packs of cigarettes smoked per day did not differ significantly between individuals with cancer (mean of 1.2 pack/day). There was no significant association between the frequency of C677T and A1298C polymorphisms in the different groups with cancer, compared to the control group. However, the T allele (C677T) occurred with higher prevalence in group $2(\mathrm{p}=0.0197, \mathrm{OR}$ $2.24,95 \%$ CI 1.18 to 4.27 ) compared to the control group.

Conclusions: The presence of polymorphisms of genes involved in the metabolism of micronutrients, as the MTHFR, can exacerbate the nutritional deficiency generated by alcohol consumption, since such genetic alterations leads to a reduction of enzymatic activity. The $\mathrm{T}$ allele is present 2.24 times more in group 2 in the subjects studied, suggesting an association with the development of this tumor despite lower alcohol consumption. In the present study, there is a tendency of association between C677T polymorphism and alcohol consumption, which requires an evaluation of a larger number of patients, since these data is incomplete.

\section{P054 \\ Excess of lodine and Dietary Sodium in Brazilian Schoolchildren: A Review of the Literature}

\author{
Danielle Eveline Quadros, Lúcia Gomes Rodrigues \\ Universidade Federal do Estado do Rio De Janeiro
}

The iodination of salt is an important factor for the reduction of iodine deficiency, for many years considered a serious public health problem worldwide, associated with damage to brain development in fetuses and children, congenital abnormalities, cretinism, goiter and hypothyroidism. However, recent research has shown an increase in urinary iodine excretion in Brazilian schoolchildren, where the average iodine excretion is above $300 \mu \mathrm{g} / \mathrm{L}$ per day and up to $600 \mu \mathrm{g} / \mathrm{L}$ per day. The expected daily urinary excretion of iodine in children in the 4-10 years range is 100 to $200 \mu \mathrm{g} / \mathrm{L}$ per day. Acording to the World Health Organization an iodine excretion greater than $300 \mu \mathrm{g} / \mathrm{L}$ per day can lead to the emergence of chronic autoimmune thyroiditis and hypothyroidism. Chronic thyroiditis is an autoimmune disorder that occurs when autoantibodies are directed against self antigens (thyroglobulin, thyroid peroxidases and thyroid-stimulating hormone receptors) responsible for stimulating the thyroid gland to produce thyroxine and triiodothyronine. It can be caused by excess iodine which can saturate tireoperoxidases receptors, and block the release of hormones from the thyroid gland, causing their progressive failure and triggering hypothyroidism. The increase in urinary iodine may be attributed to a high intake of dietary salt and certain industrialized food products, coupled with the exponential growth of the prevalence of overweight in the population, especially among children and adolescents, leading to an early emergence of autoimmune thyroiditis, in addition to hypertension, dyslipidemia, glucose intolerance, among others.

\section{P055 \\ Indicated for the Young Investigator Award \\ Docosahexaenoic Acid Regulates Genes Related with Lipid Metabolism Differently in Normal and Breast Cancer Cell Line: A Microarray Approach}

\section{Danielle Fontes Almeida, Rita de Cássia Borges Castro, Rosimeire Aparecida Roela, Tatiane Katsue F. Mazzotti, Graziela Rosa Ravacci, Dan Linetzky Waitzberg \\ Faculdade de Medicina da Universidade de São Paulo}

Background: Breast cancer is one of the most common cancers in women throughout the world. Activation of lipid metabolism is an early event in carcinogenesis and a central hallmark of many cancers. Breast cancer cells can produce significantly high amounts of fats, because of overexpression of the peroxisome proliferator-activated receptor (PPAR) $\gamma$-binding protein and the nuclear receptor NR1D1 (nuclear receptor subfamily 1 , group D, member 1, Rev-erb $\alpha$ ). These genes upregulate de novo fatty acid synthesis, which is a critical pathway for the energy production and survival of these cells. There are 
both epidemiologic and experimental evidences that the long-chain omega-3 fatty acids (specially docosahexaenoic acid -DHA), which occur at high levels in some fish oils, exert protective effects against some common cancers, especially the breast one. DHA has been shown to inhibit cancer cell growth in part by modifying cell signaling. However, the gene expression profile resulting from the action of DHA in breast cancer has not been elucidated yet.

Objectives and Methods: We aimed to examine the effects of DHA on breast cancer cell line (SKBR-3) and normal breast cell line (HB4a) using Affymetrix Human Gene 1.0 ST microarray chip. We adopted as significant $\mathrm{p}$ values lower than 0.01 , corrected by FDR (false discovery rates).

Results: We identified 174, and 126 differentially expressed genes after DHA treatment (with $100 \mu \mathrm{M}$ of DHA for 72 hours), in normal cells (HB4a), and metastatic cells (SKBR3), respectively. Notably, the molecular pathways for the differentially expressed genes included mostly those related to lipid metabolism. In the normal cell line we found overexpressed genes (PLIN2, FABP3, CD36, ANGPTL4) related with lipid metabolism, however other genes involved in lipid metabolism were down-expressed (THRSP, INSIG1, SCD, LDLR, DHCR7) in breast cancer cell line after treatment.

Conclusions: We suggest a possible mechanism of DHA effect in breast cancer by lipid metabolism control. These molecular annotations collectively contribute to a growing knowledgebase on the putative mechanisms of action of DHA in breast cancer pathogenesis.

\section{P056 \\ Influence of Metabolic Syndrome Components on Biomarkers of Oxidative Stress}

\section{Danielle Venturini, Andrea Name C. Simão, Isaías Dichi \\ University of Londrina}

Background: Metabolic syndrome (MetS) is characterized by increased adiposity, insulin-resistance, atherogenic dyslipidemia, oxidative stress, and elevated cardiovascular risk and frequently involves low-grade inflammation and hyperuricemia.

Objectives: The objective of this study was evaluated which metabolic syndrome components were related to oxidative stress markers.

Methods: The present was a cross-sectional study and evaluated 76 subjects with MetS (15 male and 61 female) aged (49.8 \pm 9.09$)$, selected among Internal Medicine ambulatory patients and healthy workers of the University Hospital of Londrina, Paraná, Brazil.

Results: Patients with increased glucose levels and hypertriacylglycerolemia showed higher advanced oxidation protein products (AOPP) $(\mathrm{p}<0.05$ and $\mathrm{p}<0.0001$ respectively) and lower total antioxidant capacity (TRAP)/uric acid ratio $(\mathrm{p}<0.05)$, whereas patients with decreased HDL-cholesterol levels demonstrated higher AOPP $(\mathrm{p}<0.05)$, Patients with increased C-reactive protein presented higher AOPP and higher hydroperoxide and hydrogen peroxide concentrations determined by ferrous oxidation-xylenol orange assay (FOX) levels $(\mathrm{p}<0.05)$, whereas patients with hyperuricemia had increased AOPP and nitric oxide $(\mathrm{NO})$ levels $(\mathrm{p}<0.05)$. Obese patients showed higher FOX levels $(\mathrm{p}<0.05)$.
Conclusions: This study provides evidence that hypertriyacylglycerolemia, hyperglycemia, low levels of HDL-C, inflammation and high levels of uric acid are closely linked to elevated systemic oxidative stress in MetS.

\section{P057 \\ Overweight and Dyslipidemia in Adolescents from the City of Londrina (PR), Brazil}

Crisieli M. Tomeleri, Danilo R.P. Silva,

Mariana S. Carnelossi, Helena Scheler, Danielle Venturini,

Alessandra M. Okino, Décio Sabbatini Barbosa,

Enio R.V. Ronque, Edilson S. Cyrino

Universidade Estadual de Londrina

Background: Overweight has grown markedly in the Brazilian population, affecting increasingly younger population. In children and adolescents being overweight body is configured as one of the main risk factors for cardiovascular disease, due to their excessive growth and strong association with other risk factors.

Objectives: To evaluate the association between overweight and dyslipidemia in a representative sample of adolescents from public schools from the city of Londrina- PR, Brazil.

Methods: Cross sectional study with 1024 adolescents aged 14 to 17 years, both sexes, attending public schools in the city of Londrina / PR. For the characterization of overweight, we adopted the values of body mass index (BMI) according to the cutoff points proposed by Cole et al. (2000). The presence of dyslipidemia was characterized by a change in the variable components of the lipid profile (total cholesterol, HDL-cholesterol, LDL-cholesterol and triglycerides) after $12 \mathrm{~h}$ of fasting, following the reference values of $\mathrm{R}$ Guidelines for the Prevention of Atherosclerosis in Childhood and Adolescents (SBC, 2005). For statistical analyzes, we applied initially Pearson $\mathrm{x} 2$ test to determine possible associations between nutritional status and dyslipidemia $(\mathrm{P}<0.05)$. Subsequently, the multivariate model was structured with the binary logistic regression, with adjustment of potential confounders (age, sex, maturation and physical activity level).

Results: Analyses revealed that dyslipidemias were significantly associated with nutritional status ( $\mathrm{P}<0.05)$, except only to dyslipidemia characterized by high total cholesterol $(\mathrm{P}=0.31)$. Thus, after regression analysis was observed that the LDL-C $[\beta($ IC95\% $)=1.45$ $(1.01-2.08)], \quad H D L-C \quad[\beta(\mathrm{IC} 95 \%)=4.52$ (3.11-6.53)], TG $[\beta(\mathrm{IC} 95 \%)=5.56(2.61-11.83)]$ was independently related to nutritional status.

Conclusions: These results suggest that the nutritional status was significantly associated with the majority of dyslipidemia, even after adjustment for confounding variables, and thus, it is recommended that preventive measures are taken to prevent more children and adolescents will become obese increases the risk of acquiring cardiovascular risk factors.

Financial support: CNPQ E CAPES. 
P058

\section{Oxidative Stress in Overweight Subjects with or without Metabolic Syndrome}

Danielle Venturini, Nicole A. Scripes, Petrônio A.S. Melo,

Francine M. Belinetti, Marcell A.B. Lozovoy,

Tathiana N.C. Simão, Andrea N.C. Simão, Isaías Dichi

University of Londrina, University of North Paraná

Background: Metabolic syndrome (MetS) consists of pathological conditions including insulin resistance, arterial hypertension, obesity and dyslipidemia, which accelerate atherosclerosis and increase cardiovascular disease risk. Although several studies have been performed to verify the role of oxidative stress in obese subjects with MetS, few studies have reported its role in overweight subjects.

Objectives: The aim of this study was to verify the influence of metabolic syndrome on lipid and protein oxidation and on antioxidant defenses in overweight subjects.

Methods: This cross sectional study was performed with 123 subjects ( 50 controls and 73 overweight). The control group included 50 healthy individuals (body mass index - BMI between 20 and 24.9 $\mathrm{kg} / \mathrm{m}^{2}$ ) and without metabolic syndrome (MetS). Overweight group consisted of 73 subjects (BMI between 25 and $29.9 \mathrm{~kg} / \mathrm{m}^{2}$ ). Overweight subjects were divided into two groups: with MetS (29 subjects) and without MetS (44 subjects).

Results: Control group and overweight subjects without metabolic syndrome showed no differences in the oxidative stress parameters and total antioxidant capacity (TRAP). Overweight subjects with metabolic syndrome had higher hydroperoxide concentrations measured by chemiluminescence compared to the control group $(\mathrm{p}<$ 0.05 ), higher hydroperoxide and hydrogen peroxide concentrations determined by ferrous oxidation-xylenol orange assay compared to overweight subjects without metabolic syndrome $(p<0.001)$, and higher advanced oxidation protein products (AOPP) concentrations $(\mathrm{p}<0.001)$ compared to the other groups. AOPP was directly correlated with uric acid concentrations. Overweight subjects with metabolic syndrome had lower TRAP concentrations compared to the control group ( $\mathrm{p}<0.001)$, when TRAP was corrected by to uric acid concentrations.

Conclusions: This study showed that the presence of metabolic syndrome is the main determinant for the redox imbalance, characterized by increased plasma oxidation and reduced antioxidant capacity, verified in overweight subjects.

Financial support: FAEPE.

\section{P059 \\ Indicated for the Young Investigator Award The IFAT-1 Mouse Model: Studying Temporal and Tissue Specific Effects of n-3 Polyunsaturated Fatty Acid Enrichment}

Shannon E. Clarke, Jing X. Kang, David W.L. Ma

University Of Guelph/ MGH Harvard Medical School

Background: Clarification of the role of dietary n-3 polyunsaturated fatty acids (PUFA) for the prevention and treatment of chronic human diseases is of great research interest. The fat- 1 transgenic mouse, which endogenously synthesizes n-3 PUFA from n-6 PUFA, provides a unique framework to specifically investigate the molecular and cellular mechanisms by which n-3 PUFA affect cell function. To date, research employing the fat-1 mouse model has provided invaluable insight into the chemo-, cardio- and neuro-protective effects of lifelong n-3 PUFA enrichment. However, a detailed understanding of the mechanisms by which n-3 PUFA temporally regulate health related outcomes is lacking. Therefore, an inducible fat-1 (ifat-1) mouse model, which carries a Cre recombinase dependent version of the fat-1 transgene, has recently been developed.

Objectives: To determine the utility of the ifat- 1 transgene as a model for conditional n-3 PUFA enrichment.

Methods: To screen transgene function in-vitro, HEK293T cells were co-transfected with the ifat-1 transgene and either, a Cre expression plasmid or a control empty vector. For in-vivo analysis, ifat-1 transgenic females were bred with R26-Cre-ER ${ }^{\mathrm{T} 2}$ mice, a tamoxifen inducible Cre expression model. All mice were fed a $10 \%$ safflower diet. At 6 weeks of age ifat-1/R26-Cre-ER ${ }^{\mathrm{T} 2}$ double hybrid males $(\mathrm{n}=$ 2-3/group) were transiently treated with either tamoxifen or vehicle (corn-oil). Tissues were collected at 9 weeks of age. Total lipids were extracted and phospholipid fatty acid composition was determined using gas chromatography.

Results: Co-transfection of cells with ifat- 1 and Cre significantly reduced the n-6/n-3 PUFA ratio in major phospholipid fractions, phosphatidylethanolamine (PE, $-91 \% \mathrm{p}<0.01)$ and phosphatidylcholine $(\mathrm{PC},-98 \% \mathrm{p}<0.01)$. Similarly, tamoxifen treated double hybrid males had a lower $\mathrm{n}-6 / \mathrm{n}-3$ ratio in the liver $(\mathrm{PE},-73 \% \mathrm{p}<0.05, \mathrm{PC}$, $-58 \% \mathrm{p}<0.01$ ), kidney (PE, $-58 \% \mathrm{p}<0.01, \mathrm{PC},-58 \% \mathrm{p}<0.01)$ and muscle (PE, $-51 \% \mathrm{p}<0.05, \mathrm{PC},-48 \% \mathrm{p}<0.01)$ relative to vehicle treated controls. Within each of these tissues the major n-3 PUFA enriched and n-6 PUFA reduced was 22:6n3 and 22:5n6, respectively. Total saturated, monounsaturated and PUFA did not differ between groups.

Conclusions: The ifat-1 plasmid and transgenic mouse have potential application to address the temporal and/or tissue specific effects of n-3 PUFA in disease prevention and treatment. 


\section{P060 \\ Indicated for the Young Investigator Award Stimulation of Muscle Growth and Carbohydrate Metabolism Genes by Dietary Brassinosteroids}

\author{
Debora Esposito, Haley Holway, Slavko Komarnytsky \\ North Carolina State University
}

Background: Muscle mass and strength are progressively lost during natural aging (sarcopenia) or disease (dystrophy). There is no uniform strategy to prevent or treat muscle loss. Our previous studies have shown that plant-specific polyhydroxylated derivatives of 5a-cholestane (brassinosteroids) stimulate protein synthesis and myogenic stem function in skeletal muscle cell culture by yet unknown mechanism(s) that may involve Akt/mTOR and Akt/FOXO signaling pathways.

Objectives: The present study was designed to determine effects of brassinosteroid supplementation on measures of myogenic and metabolic transcriptional profiles in healthy rat model.

Methods: Three groups of 6 wk old male Wistar rats $(n=6)$ fed normal diet (protein content $23.9 \%$ ) were gavaged daily for $24 \mathrm{~d}$ with $1 \mathrm{ml}$ of vehicle (5\% DMSO in corn oil), 20 or $60 \mathrm{mg} / \mathrm{kg}$ body weight of homobrassinolide (HB) naturally found in cabbage and mustards. Body composition was assessed by dual-energy X-ray absorptiometry, while muscle samples were subjected to the pathway-specific qPCR array analysis.

Results: Oral administration of $\mathrm{HB}$ to healthy rats increased food intake, body weight gain, lean body mass, and gastrocnemius muscle mass as compared with vehicle-treated controls. Three subsets of genes were expressed higher in skeletal muscles of HB-treated animals than in the controls including PI3K/Akt signaling pathway (Adra1d, Igfbp1, Srebf1), carbohydrate metabolism and growth (Fbp2, Igf2), and myogenic differentiation (Myod1, Myf5, Myf6, Myog). The R1d adrenergic receptor Adrald known to mediate endogenous functions of catecholamines showed the highest upregulation in response to the treatment ( 6.5 fold).

Conclusions: The skeletal muscle gene expression profile is positively modified by dietary $\mathrm{HB}$ and reveals the adrenergic receptor R1d (Adrald) gene as a novel biomarker for muscle survival and differentiation. These findings underscore the importance of dietary brassinosteroids as putative agents for prevention and treatment of muscle loss.

\section{P061}

\section{High Fructose Diet Stimulates Neutrophil Recruitment to Hepatic and Adipose Tissues in the Postprandial State}

\author{
Débora Fernandes Rodrigues, Pedro Elias Marques, \\ Jaqueline Pereira Lana, Gustavo Batista Menezes, \\ Mauro Martins Teixeira, Adaliene Versiani M. Ferreira \\ Universidade Federal de Minas Gerais
}

Background: Recent decades have witnessed an enormous rise in fructose consumption, a carbohydrate massively used in the food industry due to its sweeter taste and low cost. Some evidences associate the increased consumption of fructose with growth rates of obesity and metabolic syndrome. In fact, the chronic consumption of this carbohydrate increases visceral adipose deposition and de novo lipogenesis and also induces dyslipidemia, insulin resistance and a state of low-grade chronic inflammation. A previous study of our laboratory have shown that animals fed a fructose-rich diet presented a systemic proinflammatory state and high levels of cytokines in liver and adipose tissue.

Objectives: To investigate whether a diet rich in fructose increases neutrophil recruitment in metabolically active organs, specifically liver and adipose tissue, in the postprandial state.

Methods: The experimental protocol was approved by the "Ethics Committee in Animal Experimentation at the Universidade Federal de Minas Gerais" (protocol No: 63/2010). Male Lysm-eGFP mice (green fluorescent neutrophils) at $10 \mathrm{w}$ of age were housed in individual cages for $15 \mathrm{~h}$ in fasting and divided into 2 groups: control diet, AIN-93 diet with $20 \%$ of sucrose, and high fructose diet, AIN-93 diet with $20 \%$ of fructose. Animals were fed for $1 \mathrm{~h}$ with $1 \mathrm{~g}$ of the respective diets. After $2 \mathrm{~h}$ postprandial, mice were anesthetized with ketamine $(130 \mathrm{mg} / \mathrm{kg})$ and xylazine $(0.3 \mathrm{mg} / \mathrm{kg})$ and injected i.v. with the fluorophore Rhodamine $6 \mathrm{G}(0.5 \mathrm{mg} / \mathrm{mL})$ for hepatocyte and adipocyte visualization. The liver and epididymal adipose tissue were exposed and the microcirculation was visualized under confocal microscopy. After that, animals were euthanized by cervical dislocation and samples of liver were collected and stored at $-20{ }^{\circ} \mathrm{C}$ for Myeloperoxidase (MPO) analysis.

Results: Mice fed control diet showed a small number of adhered neutrophils through the imaging time in liver and adipose tissue. However, animals fed the fructose-rich diet exhibited a high flow of neutrophils in blood vessels of both tissues. MPO assay demonstrated a high activity of this enzyme in animals fed the fructose-rich diet $(0.79 \pm 0.03)$ compared with the control group $(0.52 \pm 0.05)$.

Conclusions: Our results support the idea that a diet rich in fructose induces an acute proinflammatory response which may lead to recruitment of immune cells in the postprandial state to key metabolic organs involved in the development of obesity and associated diseases. 


\section{P062 \\ Indicated for the Young Investigator Award Influence of Yerba Mate (Ilex Paraguariensis) in Insulin Signaling Pathway}

Demetrius Paiva Arçari, Lucio F. Caldas Ferraz, Juliana Carvalho Santos, Marcelo Lima Ribeiro

UNICAMP/USF

Background: Many studies have been reported that, yerba mate (Ilex paraguariensis), one of the most widely consumed plants in South America, are rich in several bioactive compounds. This studies have shown that the compounds found in yerba mate has several biological functions, such as antioxidant, anti-inflammatory, immunomodulatory, anticancer, modification of cholesterol metabolism, glucose homeostasis, loss-weight and others. Although several studies highlight the biological functions of yerba mate, there is lack of evidence providing their ability to modulate expression of genes related with insulin signaling pathway.

Objectives: The aim of this study was to evaluate in high fat diet mice the effects of yerba mate toasted in gene expression and transcription factor related with insulin signaling pathway.

Methods: Forty five mice were introduced to either standard or high fat diets (HFD). After 8 weeks on a high fat diet, mice were randomly assigned to one of the two treatment conditions, water or yerba mate extract at $1.0 \mathrm{~g} \mathrm{~kg}^{-1}$. After 8 weeks in treatment, hepatic insulin response were evaluated, liver samples were submitted a PCR screening array for 84 genes related with PI3K-AKT insulin pathway. Genes that had a modified expression pattern in PCR Array have validated by real time PCR and normalized by the expression of constitutive gene and the nuclear translocation of FOXO1 was determined by electrophoretic mobility shift assay (EMSA).

Results: This study showed that HFD causes a significant decrease of gene expression in AKT-2, IRS-1, IRS-2, PI3KCA, PI3KCG and PDK-1 involved in deferent steps of the insulin signaling pathway in liver and causes a significant increase expression of PEPCK and G6Pase the major regulators of gluconeogenesis process activated by FOXO1 transcription factor. After treatment yerba mate as able to restore this gene expression levels and decreased expression of PEPCK and G6Pase. To check nuclear translocation of FOXO1 our results showed that Yerba maté extract was able to reduce the nuclear translocation of FOXO1 in HFD mice.

Conclusions: This study show for the first time the specific action of mate on major isoforms existing in hepatic insulin signaling and gluconeogenesis process, contributing to the reversal of insulin resistance observed after treatment.

\section{P063 \\ Yerba Mate (Ilex Paraguariensis) Prevents Obesity Through Modulation of Genes Related with Adipogenesis}

\author{
Demetrius Paiva Arçari, Juliana Carvalho Santos, \\ Marcelo Lima Ribeiro \\ UNICAMP/USF
}

Background: Obesity is a problem of public health, resulting in significant morbidity and mortality. Several strategies have been used in order to reduce the total body weight, among these stands out the yerba mate. Mate beverages have been positively related with lossweight, however there are scarce studies that demonstrate the mechanisms involved in the action of yerba mate in the adipogenesis.

Objectives: The aim of this study was to evaluate in 3T3L1 cells and high fat diet (HFD) mice, the effects of yerba mate and some bioactive compounds in gene expression related with adipogenesis.

Methods: 3T3-L1 cells after differentiation were randomly assigned in two conditions: differentiation stage - with yerba mate and bioactive compounds (clorogenic acid, quercetin and rutin) and maturation stage - with the same compounds inserted after 96 hours. Triglyceride content was determined by Oil red O. Forty-five mice were introduced to either standard or HFD, after 8 weeks on a HFD, mice were randomly assigned to one of the two treatment conditions, water or yerba mate extract at $1.0 \mathrm{~g} \mathrm{~kg}^{-1}$. After 8 weeks in treatment adipose tissue were evaluated. Samples were submitted a PCR screening array for 84 genes related with adipogenesis. Genes that had a modified expression pattern in PCR Array have validated by real time PCR.

Results: The results of oil red O in 3T3-L1 cells, indicate that the compounds evaluated showed an effect in reduction of adipogenesis most significant in stage of differentiation compared to the stage of maturation of adipocytes, however this effect is most evident in yerba mate than bioactive compounds isolated. This study showed that yerba mate decreased CREB-1, E2F1, C/EBP $\alpha$, PPAR $\gamma 2$ and increased DLK-1, GATA-3, LRP-5, SFRP-1, TCF7L2, WNT10b, WNT3a gene expression in 3T3-L1, on the other hand the amount of genes modulated by the compounds isolated was less than the regulated by yerba mate. In addition this study evaluated the same genes in mice adipose tissue. The results showed that after HFD yerba mate inhibited gene expression of CREB-1, C/EBP $\alpha$, PPAR $\gamma 2$ and enhanced DLK1, GATA2, GATA3, KLF-2, WNT10b, WNT3a, LRP5, SFRP1 and TCF7L2 anti-adipogenic genes.

Conclusions: This study show in vitro and in vivo the specific action of yerba mate and some bioactive compounds on adipogenesis. Furthermore, it was observed that the bioactive compounds in mate act by modulating different genes related to adipocyte differentiation process. 
P064

Antimicrobial Activity and Proteomic Analyses of Crude Extract of Germinated Yellow Soybean (Glycine Max)

Paula Martins Pedrote, Lemuel Araújo de Souza, Luciana dos Santos Gomes, Denise Marie Delgado Bouts, Luiza Gervazoni Ferreira de Oliveira, Elmo Eduardo de Almeida Amaral, Russolina Benedeta Zingali, Eliane Fialho

UFRJ

Introduction: Plants when exposed to injuries, produces antimicrobial compounds called phytoalexins. These compounds are present constitutively or can be induced, and thus, provide a broad spectrum of antimicrobial action. In this context, proteomic is a promising approach and has been successfully used to identify proteins of seeds in different conditions, like germination.

Objectives: Analyze the antimicrobial activity of germinated extract of yellow soybean (Glycine max) cv. BRS 258 exposed to the parasite Leishmania amazonensis and evaluate method of protein extraction for 2-D gel reference map.

Material and Methods: Soybeans were soaked in sterile distilled water and germinated for 48 hours. After that, they were crushed and solubilized in buffer Tris- $\mathrm{HCl} 30 \mathrm{mM} \mathrm{pH} \mathrm{8.0.} \mathrm{The} \mathrm{L.} \mathrm{amazonen-}$ sis promastigote (MHOM/BR/77/LTB0016 strain) were grown in Schneider medium, $\mathrm{pH}$ 7.2. The parasites were kept in an incubator at $26^{\circ} \mathrm{C}$. After the promastigotes cells reached $1 \times 106$ cells $/ \mathrm{ml}$ concentration, they were cultured in the absence or presence of different concentrations of samples for a period of 96 hours. In order to obtain the bidimensional gel, proteins were extracted with TCA/acetone, Tris$\mathrm{HCl}$ and thioureia/ureia procedures. After gel digestion with trypsin, proteins were subsequently identified by MALDI-TOF/TOF.

Results: Germinated extract of BRS 258 inhibited $80 \%$ of Leishmania amazonensis growth. In addition thiourea/urea method showed enhanced solubilization of proteins and higher protein resolution and spot intensity. Also, proteins involved in developmental were most abundant.

Conclusions: Results showed that proteins involved in embryonic developmental have antileishmania activity. Furthermore, reference map could provide information on the biosynthesis of natural products with biological activity in soybean.

Financial support: CAPES, FAPERJ.

\section{P065}

In Silico Analyses of Human Genes Involved in the Diabesin Triad (Diabetes, Obesity and Inflammation) and their Association to Imprinted Genes

\author{
Ana Paula Moreira Bezerra, Samara Cardoso Silva- \\ Santiago, Elton José Rosas Vasconcelos, \\ Ana Carolina Landim Pacheco, Monica Moraes Silva, \\ Diana Magalhaes Oliveira \\ Universidade Estadual do Ceara - UECE
}

The term diabesity has been used to describe concomitant morbid conditions of obesity, insulin resistance (IR), metabolic syndrome (MetS) and type 2 diabetes (T2DM). Here we have coined the term "diabesin" to also include the inflammation response. A key genetic process, genomic imprinting, is also interesting to consider on diabesin, since a relatively small number of human genes are imprinted and they are recognized as important players of major gene-environment interactions.

Objectives: Aiming to identify a panel of human genes implicated in diabesin, here we have attempted to associate imprinted genes that might serve as potential indicators for establishing potential risk haplotypes for this increasingly prevalent condition. A haplotype is a combination of alleles or of single nucleotide polymorphisms (SNPs) tending to be inherited together.

Methods: Bioinformatics analyses involved exhaustive genomic searches, performed using queries not limited to "genomic imprinting," "imprinted", "obesity", "inflammation", and "diabetes". A gene catalog was compiled, for each imprinted or predicted-to-be imprinted gene, we extracted annotations, references, and sequences by performing comprehensive searches in relevant databases. Association features of the studied conditions, not found in existing databases, were obtained by analyzing related publications.

Results: We have compiled over 80 unique human genes that could be unambiguously associated to diabesin. They have been, then, divided in subgroups to comprise a full repertoire of diabesin gene features (gene product and architecture, sequence length and assembly, SNP content, copy number variant, etc).

Conclusions: Regardless of all obvious (and not underestimated) environmental influences, a so-called "diabesin" risk haplotype would, indeed, be an ideal genomic profile to be searched for and this is the main, preliminary contribution of the present work.

Financial support: CNPQ (560264/2010-5).

\section{P066 \\ Bioinformatic Tool for User Friendly Large Scale Genotyping with SNP-Arrays}

Diego Martinez Salvanha, Ricardo Zorzetto N. Vêncio

University of Sao Paulo

One of the most promising tools in nutrigenomics, capable of investigating personal genetical configurations and, therefore, provide the link between phenotype and genotype, is the SNP-array plat- 
form. This technology allow researchers to interrogate several SNPs and other polymorphism simultaneously and laid the foundation of high throughput, low cost, sample rich genotyping, an emerging field know as variomics. However, this promise can only be delivered if variomics meets nutrigenomics mediated by bioinformatics. Bioinformatics covers all aspects of acquisition, processing, storage, distribution, analysis and interpretation of biological information through a combination of procedures and techniques from mathematics, statistics and computer science. In the present work we present our groups effort to deploy a bioinformatics framework aiming biologists whose research may take advantage of high throughput genotyping data and have no special training in computational sciences. The general goal of preprocessing and analyzing SNP arrays data is to normalize and summarize feature intensities to predict the genotype of each allele of a SNP interrogated. Existing methodologies are responsible for performing genotyping accuracy well over $99.5 \%$ with $99.9 \%$ of reproducibility - however they are not user friendly. Therefore, we were motivated to provide such accurate methods in a one-stop-shop software integrated into consolidated frameworks, such as Gaggle Genome Browser or Gaggle-boss (http://gaggle.systemsbiology.net). We developed an integrated software that allows extraction from SNP-array technology based on usability, in a transparent and automated way for the end user. This software was developed using Java Language and can be briefly described as: (i) use of gaggle-boss source-code to communicate and integrate the Gaggle Genome Browser tool, (ii) use of rJava to integrate R-language with this developed software. All implementation are freely available at http://labpib.ffclrp.usp.br/ dmartinez/base/. Understanding that the availability of tools is key to the proper development of a scientific field, we hope to contribute to efforts in nutrigenomics providing the means to make variomics accessible to a broader community.

Financial support: FAPESP/CAPES.

\section{P067 \\ Effects of Undernutrition During Pregnant and Lactation on Glucose Metabolism, Insulin Signaling, and Global DNA Methylation in Skeletal Muscle from Male Offspring}

\author{
Diogo Antonio Alves Vasconcelos, \\ Sandro Massao Hirabara, Carlos Hermano Justa Pinheiro, \\ Renato Tadeu Nachbar, Carlos Flores Rodrigues Junio, \\ Amanda Rabello Crisma, Cátia Lira Do Amaral, \\ Phablo Sávio Abreu Teixeira, Adriano Bento-Santos, \\ Filippe Tebas-Falcão, Alice Cristina Rodrigues, \\ Carol Gois Leandro, Rui Curi, Tania Cristina Pithon-Curi
}

University of São Paulo

Deficiency of nutrients in critical phases of growth and development, including gestation and lactation, promotes short- and longterm effects on health. Protein undernutrition during gestation, lactation, and childhood predisposes the adults for cardiovascular diseases, type II diabetes mellitus, dislypidemias and obesity. Skeletal muscle has fundamental function on development and worsening of these effects. For example, alteration in insulin pathway in skeletal muscle tissue results in important changes in glucose metabolism. Therefore, the objectives of this work were to investigate the effects of undernutrition during pregnant and lactation on glucose metabolism, insulin signaling, and global DNA methylation in skeletal muscle from male offspring. Wistar rats were submitted to low protein diet $(8 \%$ protein) during gestation and lactation. Body weight was significantly decrease at the $21^{\circ}$ day: $56.16 \pm 2.30$ for control group (co) and $32.72 \mathrm{~g} \pm 1.04$ for undernutrition group $(\mathrm{dn}), 58.16 \%$ of difference $(\mathrm{p}<0.001)$. But, body weight at 7 months was not different (co 417.1g \pm 15.2 vs dn 412.8g \pm 12.90). Insulin-stimulated glucose uptake and metabolism (oxidation and glycogen synthesis) was decreased in undernutrition group: co $4.47 \pm 0.33$ vs $2.38 \pm 0.33$ (p < $0.01)$ for glucose uptake, co $0.604 \pm$ vs $\operatorname{dn} 0.295 \pm(\mathrm{p}<0.001)$ for glucose oxidation, and co $2.00 \pm$ vs dn $1.471 \pm(\mathrm{p}<0.05)$. Akt phosphorylation was increased by insulin (co- $9.48 \pm 1.50 \mathrm{vs} \mathrm{co}+23.23 \pm$ $1.66, \mathrm{p}<0.001$ ) and (nd $-7.77 \pm 1.89$, nd $+15.48 \pm 2.32, \mathrm{p}<05)$, but undernutrition decreased the insulin-stimulated Akt phosphorylation when compared to control group. Total Akt protein content was also reduced by undernutrition (co- $7.30 \pm 1.00 \mathrm{vs} \mathrm{dn}-4.02 \pm 0.76 \mathrm{p}<$ $0.05)$ and $(\mathrm{co}+7.40 \pm 0.5$ vs nd $+3.09 \pm 0.88 \mathrm{p}<0.05)$. However, GSK- $3 \beta$ protein was not modified by undernutrition, but it was only phosphorylated by insulin in the control group (co- $15.43 \pm 2.96$ vs co $+26.12 \pm 1.46 \mathrm{p}<0.05)$. Global DNA methylation in the EDL muscle was not modified. We concluded that the undernutrition in early phases was able to induce insulin resistance at adult phase in rat skeletal muscle associated with decreased expression and phosphorylation of the Akt protein, without modification of global DNA methylation.

Financial support: CNPQ.

\section{P068 \\ The Role of Macadamia Oil Supplementation on Insulin Sensitivity in the Skeletal Muscle of Mice Fed with a High-Fat Diet. The Role of Palmitoleic Fatty Acid?}

Edson Alves de Lima Junior, José Cesar Rosa Neto, Rodrigo Xavier das Neves, Sandro Massao Hirabara, Marília C. Leite Seelaender, Rui Curi

Institute of Biomedical Sciences, USP

Background: Obesity is one of the diseases most associated with the modern lifestyle and has a range of comorbidities as a consequence. In turn, the consumption of some fatty acids, mono or polyunsaturated, seem to bring a benefit on insulin sensitivity. High content of lipids in the diet is associated with insulin resistance. The knowledge on the effect of palmitoleic acid ( $\omega-7)$, in the mechanism of action involved on the insulin sensitivity in the skeletal muscle is still incipient. With that, this project is aimed at investigating the role of the supplementation with macadamia oil, on the insulin resistance induced by a high-fat diet.

The objective of this research was to investigate the role of supplementation of macadamia oil, rich in palmitoleic acid, on insulin sensitivity (systemic and muscle) in mice of the C57BL / 6.

Methods: The animals received a balanced diet, supplemented (CTMAC) or not with macadamia oil (CTH2O) during 12 weeks 
(weeks 0 to 12). The animals received a balanced diet during the first 4 weeks, from the weeks 5 to 12 , a group of animals continued receivinga balanced diet and the other group received a high-fat diet (HFH2O/HFMAC). For that, the following parameters were analyzed: systemic sensitivity to insulin estimated in vivo by the insulin tolerance test (iTT), glucose tolerance test (GTT), basal glycemia and insulinemia. The in vitro experiments (glucose uptake) were carried out in isolated soleus muscle.

Results: Fasted glucose of CTMAC animals were statistically lower for both hyperlipidic diet $(p<0.001)$, while animals CTH2Oonly to groupHFH2O $(\mathrm{p}<0.05)$. The basal insulin showed no significant difference between the groups. High-fat diet was able to induce a decreased glucose tolerance $(\mathrm{p}<0.001)$, being the supplementation unable to lead to statistically significant differences in GTT.However, the AUC of the iTT shows that the CTMAC group have a greater sensitivity to insulin for both high fat diet $(\mathrm{p}<0.01)$, whereas the group $\mathrm{CTH} 2 \mathrm{O}$ showed no change. After stimulation by insulin, only group CTMAC has increased glucose uptake for all other groups $(\mathrm{p}<0.05)$.

Conclusions: Our data suggest potential benefits of supplementation effects of the macadamia oil (rich in $\omega-7$ ) on insulin sensitivity, a possible effect mediated by increased glucose uptake stimulated in animals that received the control diet.

Financial support: FAPESP (2011/14026-6).

\section{P069}

\section{The Role of Macadamia Oil Supplementation on Morphometric Characteristics of Adipocytes}

\author{
José Cesar Rosa Neto, Edson Alves de Lima Junior, \\ Rodrigo Xavier das Neves, Sandro Massao Hirabara, \\ Marília C. Leite Seelaender, Rui Curi \\ Institute of Biomedical Sciences, USP
}

Background: Obesity is one of the diseases most associated with the modern lifestyle and has a range of comorbidities as a consequence. Indeed, the appearance of adipocytes large diameter is closely related to insulin resistance and secretion of adipokines. The knowledge on the effect of palmitoleic acid ( $\omega-7)$, and their role on the morphometric characteristics of adipocytes is still incipient.

The objective of this research was to investigate the role of supplementation of macadamia oil, rich in palmitoleic acid, over the area and average diameter adipocyte of mice C57BL / 6.

Methods: The animals received a balanced diet, supplemented or not with macadamia oil (CTMAC/CTH2O) during 12 weeks. The animals supplemented or not with macadamia oil (HFH2O/HFMAC) received a balanced diet during the first 4 weeks and from 5 to 12 week received high fat diet. For morphometric analysis the tissue sections was stained with hematoxylin and eosin and morpholmetrical analysis was performed using Image Pro-Plus 6.0.

Results: The administration of a diet with high content of lipids, as expected, was able to increase the final weight of retroperitoneal adipose tissue $(p<0.001)$, however supplementation was ineffective in attenuating the mass of adipose pads. The histology confirmed the hypertrophic effect on adipose cells when there is exposure to high fat diet, only in the group HFH2O $(\mathrm{p}<0.05)$. There was no significant difference over the area and mean diameter of adipocytes between the control groups. Interestingly HFMAC group appears to have a attenuate effect on these morphological properties, since there is no statistical difference for the both control groups $(p>0.05)$. Indeed we verified the distribution of adipocytes smaller in this group.

Conclusions: Our data suggest potential effects of supplementation of the macadamia oil (rich in $\omega-7$ ), on attenuate the size of adipocytes of animals fed high-fat diet and supplementation.

Financial support: FAPESP (2010/15124-9).

\section{P070 \\ Dietary PUFAS Interact with UGT1A1 Promoter Genotype to Determine Serum Bilirubin Levels of Healthy Women in Child- Bearing Age}

Eduardo de Carli, Carina de Fátima Rodrigues

Universidade Federal de Pelotas

Background: Bilirubin is an $\mathrm{Hb}$ degradation product that has cardiovascular protective properties. UGT1A1 is the major gene controlling serum bilirubin levels. The presence of a Timine Adenine (TA)-repeat polymorphism in the gene's promoter, an allele designated UGT1A1*28, causes a lower gene activity and higher serum bilirubin levels. Dietary PUFAs also has been linked to the reduction in cardiovascular disease risk, however, the physiological mechanisms behind this association are yet not full elucidated.

Objectives: Our objective was to determine, in an observational study, whether habitual dietary PUFAs intake is associated with serum bilirubin levels of healthy women in childbearing age with different UGT1A1 promoter genotypes.

Methods: One hundred sixteen healthy Caucasian women $(21 \pm$ 3 years old) participated in a Portuguese cohort study that investigate genetic and non-genetic determinants factors of bilirubin levels. A validated quantitative food-frequency questionnaire was used to estimate habitual dietary PUFAs intake. These data were adjusted for energy and analyzed as continuous (g/day) or categorical variable (tertiles of intake). Statistical analyses was performed by one wayANOVA test, simple linear correlations and general univariate regression modeling adjusting for $\mathrm{Hb}$ levels and total dietary fat intake using the SPSS software (version 16).

Results: Mean PUFAs intake was not different between genotype groups. Independently of other variables, a significant positive correlation between PUFAs and serum bilirubin levels was observed in the group of wild homozygotes $(r=0.293, P=0.024)$, but not in heterozygote or homozygote UGT1A $1 * 28$ carriers. A significant interaction between UGT1A1 genotype and PUFAs intake was found $(\mathrm{P}=0.037)$, women with wild genotype who consumed the higher tertile of PUFAs had $\sim 10 \%$ higher serum bilirubin than those with the same genotype at the lower tertile $(\mathrm{P}=0.087)$.

Conclusions: Our data indicate that higher dietary PUFAs intake is associated with increased bilirubin levels in women with homozygous wild UGT1A1 promoter genotype. With regard to cardiovascular diseases, these results imply that UGT1A1*28 non-carriers women may have greater possibility to augment their 
cardiovascular protection through dietary intervention. Further studies are necessary to elucidate the role of dietary PUFAs in the UGT1A1 activity modulation, taking account the effect of different UGT1A1 promoter genotypes.

Financial support: FCT AND FSE (SFRH/BD/42791/2007).

\section{P071 \\ Role of Sulforaphane in the Reduction of Oxidative Stress and Cancer Chemoprevention}

\author{
Eduardo Emanuel Sátiro Vieira, \\ Vanessa Brito Lira de Carvalho, \\ Ana Karolinne da Silva Brito, Ágatha C. Silva de Carvalho, \\ Larisse Monteles Nascimento, \\ Gabriela De Sousa Silva Rios, Kyria Jayanne Clímaco Cruz, \\ Dilina do N. Marreiro \\ Universidade Federal do Piauí
}

The phytochemicals in foods with chemopreventive activity have been of great interest as an alternative to help control cancer. Sulforaphane (SNF) is an isothiocyanate found in cruciferous vegetables, especially broccoli and broccoli sprouts. This compound was considered as a potential anticancer agent for promoting the inhibition of cytochrome P450 monooxygenases - and the reduction of oxidative stress, by inhibiting phase I enzymes involved in the carcinogenic activation and the induction of enzymes of phase II detoxification, such as glutathione S-transferase and UDPglucuronosyltransferase. Some studies show the ability of sulforaphane to modulate the Nrf2-Keap1 pathway, to induce cell cycle arrest in G2 phase by the regulation of mitogen-activated protein kinase dependent on cyclin- cdc2 or p34cdc2, to stimulate the process of apoptosis in cancer cells, probably through the family of proapoptic proteins Bcl-2 such as Bax and Bak, and inhibit tubulin polymerization and the activity of histone deacetylase. It is suggested that sulforaphane may act as a 'suppressor' during the post-initiation stages of the carcinogenesis process. This review shows the importance of sulforaphane on molecular mechanisms involved in chemoprevention, which highlights its role as a promising bioactive compound in foods in inhibiting the development and progression of various cancers.

\section{P072 \\ Hydroxytyrosol Suppresses MMP-9 and COX-2 Activity and Expression Through PKCA and PKCß1 Inhibition in Human Monocytes}

\author{
Egeria Scoditti, Marika Massaro, Nadia Calabriso, \\ Carlo Storelli, Raffaele De Caterina, \\ Maria Annunziata Carluccio \\ National Research Council (CNR) IFC
}

Background: Hydroxytyrosol (HT), the major olive oil antioxidant polyphenol in cardioprotective Mediterranean diets, is endowed with anti-inflammatory and anti-atherosclerotic activity. The production of cyclooxygenase (COX)-2-dependent inflammatory eicosanoids and the functionally linked release of matrix metalloproteinase (MMP)-9 by macrophages likely contribute to plaque instability leading to acute coronary events.

Objectives: Since both MMP-9 and COX-2 are redox-regulated, we studied the effects of HT on MMP-9 and COX-2 activity and expression in activated human monocytes, and explored underlying mechanisms.

Methods: Human peripheral blood mononuclear cells (PBMC) and U937 cells were treated with $1-10$ micromol/L HT before activation with phorbol myristate acetate (PMA). MMP-9 levels in the culture medium were determined by gelatin zymography and ELISA, while MMP-9 and COX-2 cell protein and mRNA expression were assessed by Western blotting and real time PCR, respectively. Prostaglandin (PG)E2 production and nuclear factor(NF)- $\mathrm{KB}$ activation were measured by an enzyme immunoassay, while protein kinase $\mathrm{C}$ (PKC) membrane translocation, an index of activation, was determined by Western blotting.

Results: HT reduced PMA-induced MMP-9 release, as well as cell protein and mRNA expression with an IC50 $=10$ micromol/L, without affecting tissue inhibitor of metalloproteinases (TIMP)-1. Correspondingly, HT blunted PMA-induced MMP-9 enzymatic activity and macrophages gelatin invasion. Moreover, HT inhibited PMA-induced PGE2 production and COX-2 expression, without affecting COX-1. These effects were mediated by inhibition of transcription factor NF- $\mathrm{NB}$ and PKCalpha and PKCbetal activation. Despite the ability of exogenous PGE2 to induce MMP-9, the observed HT suppression of MMP-9 was accounted for by both COX-2-dependent and -independent pathways.

Conclusions: HT, at nutritionally relevant concentrations, blunts MMP-9 and COX-2 expression and activity in activated human monocytes, thus featuring novel anti-inflammatory and plaque-stabilizing effects. Such results contribute to explaining the vascular protective effects by olive oil in Mediterranean diets. 


\section{P073 \\ Oleic Acid and Hydroxytyrosol Restore TNF- $\alpha$-Reduced Adiponectin Expression in Adipocytes: Protective Effects of Olive Oil Components in Obesity-Related Inflammation}

\author{
Egeria Scoditti, Marika Massaro, Mariangela Pellegrino, \\ Nadia Calabriso, Carlo Storelli, Maria Annunziata Carluccio, \\ Raffaele De Caterina \\ National Research Council (CNR), IFC
}

Background: Obesity is associated with increased risk of cardiometabolic disorders. Excess adiposity is accompanied by chronic inflammation and oxidative stress, with increased production of proinflammatory cytokines including tumor necrosis factor (TNF)- $\alpha$ and dysregulated secretion of adiponectin, an adipokine with insulin-sensitizing, anti-inflammatory and anti-atherosclerotic properties. The olive oil antioxidant phenol hydroxytyrosol (HT) and the MUFA oleic acid (OA, 18:1 n-9 cis) possess anti-inflammatory and vasculoprotective properties.

Objectives: We examined HT and OA effects on TNF- $\alpha$-reduced adiponectin expression in murine 3T3-L1 adipocytes and in human Simpson-Golabi-Behmel syndrome (SGBS) adipocytes, a cell strain that derives from subcutaneous white adipose tissue and behaves similarly to human primary adipocytes.

Methods: Differentiated adipocytes were treated with 100 $\mu \mathrm{mol} / \mathrm{L} \mathrm{OA}$ for $24 \mathrm{~h}$ or $0.1-10 \mu \mathrm{mol} / \mathrm{L}$ HT for $1 \mathrm{~h}$ before $24 \mathrm{~h}$ stimulation with $10 \mathrm{ng} / \mathrm{mL} \mathrm{TNF}-\alpha$. Adiponectin protein levels in the culture medium, cell protein and mRNA expression were measured by ELISA, Western blotting and real time PCR, respectively. Peroxisome proliferator-activated receptor (PPAR) $\gamma$ protein and mRNA levels as well as PPAR $\gamma$ activation were assayed by Western blotting, real time PCR, and ELISA. Finally, c-Jun N-terminal kinase (JNK) phosphorylation and reactive oxygen species (ROS) production were detected by Western blotting and a fluorescent assay, respectively.

Results: Pre-treatment with OA or HT, alone and - even more in combination, prevented TNF- $\alpha$-induced suppression of adiponectin secretion as well as cell protein and mRNA levels in both murine and human adipocytes (TNF- $\alpha$ : $40 \%$ vs control, HT or OA: $80 \%$ vs control, $\mathrm{P}<0.05$ ). HT and OA also prevented (by $30 \%$ ) TNF- $\alpha-$ induced downregulation of PPAR $\gamma$, a master regulator of adiponectin transcription, in association with blunted basal and TNF- $\alpha$-induced ROS production. Since the activation of JNK is crucial for both adiponectin and PPAR $\gamma$ suppression by TNF- $\alpha$, we explored HT and OA effect on TNF- $\alpha$-induced JNK phosphorylation: here HT or OA significantly attenuated TNF- $\alpha$-mediated JNK phosphorylation.

Conclusions: OA and HT, at nutritionally relevant concentrations, can prevent adiponectin downregulation in inflamed adipocytes via the attenuation of ROS production and JNK-mediated PPAR $\gamma$ suppression. These effects may contribute to the metabolic and vascular beneficial role of olive oil in Mediterranean diets.

\section{P074 \\ Indicated for the Young Investigator Award Carbon Metabolism Genomic Changes Are Caused by Reduced ATP Production in Biotin Starvation}

\author{
Estefania Ochoa-Ruiz, Alain de J. Hernandez-Vazquez, \\ Rodrigo Diaz-Ruiz, Daniel Ortega-Cuellar, Salvador Uribe- \\ Carvajal, Antonio Velazquez-Arellano \\ Universidad Nacional Autonoma de Mexico
}

We have previously found ATP deficit in biotin starvation (BtDEF), with changes in carbon metabolism gene expression.

Objectives: To investigate the mechanisms of energy and genetic expression changes in biotin deficiency.

Methods: Rats were made biotin deficient with an avidin containing diet. TCA cycle anaplerosis was determined by substrate enrichment of 13C-pyruvate. Isolated liver mitochondria respiratory capacity (incubated with glutamate, malate and succinate) was measured by polarography. Citochrome oxidase c activity was assayed with TMPD and ascorbate as substrates. Concentration of respiratory chain citochromes $\mathrm{cc} 1, \mathrm{~b}$ and aa3 was calculated in isolated mitochondria by differential spectra. Number of mitochondria was determined with the activity of citrate synthase and the expression of the mitochondrial transcription factor (Tfam) by colorimetry and western blots, respectively in hepatocytes isolated from BtDEF and control rats. Mitochondria capacity to produce ATP was evaluated as percentage of oxygen consumption inhibited by oligomycin in isolated hepatocytes.

Results: $13 \mathrm{C}$ was enriched in lactate and diminished in malate and glutamate in BtDEF indicating defective anaplerosis. Oxidative phosphorylation was curtailed. Mitochondrial respiratory capacity was reduced accompanied by complex IV deficiency. Concentration of citochromes cc1, b and aa3 were lower in mitochondria from BtDEF rats compared with control rats. Citrate synthase activity and Tfam expression were considerably reduced in the BtDEF hepatocytes indicating reduced quantity of mitochondria. Decreased OXPHOS was evidenced by larger inhibition of oxygen consumption by oligomycin in control hepatocytes (95\%) than in BtDEF ones $(83 \%)$, reduced ATP being associated with AMPK stimulation, and with increased transcripts of genes for $\beta$-oxidation and gluconeogenesis, and diminished ones for glycolysis and lipogenesis, in spite of sufficient glucose provision.

Conclusions: BtDEF causes ATP deficit by reduced anaplerosis and mitochondrial OXPHOS, and leads to genomic dysfunction probably by AMPK activation. 
P075

\section{Short-Term of Nutritional Overload Alters Gene Expression of Inflammatory Mediators in Visceral, Epydidimal and Retroperitonial Adipose Tissue of Wistar Rats}

Fabiane Valentini Francisqueti, André Ferreira Nascimento, Renata A. Melo Luvizotto, Camila Renata Corrêa

Universidade Estadual Paulista-UNESP Botucatu

Background: Recently has been described that proinflammatory cytokines such as TNF- $\alpha$, IKK $\beta$ and NFkB can be activated by tolllike receptors (TLR) in obesity status.

Objectives: The aim was to determine gene expression of inflammation-related gene (TNF- $\alpha$, IKK $\beta$, NFkB, TLR4) in epididymal $(\mathrm{E})$, visceral $(\mathrm{V})$ and retroperitoneal $(\mathrm{R})$ adipose tissue of rats under short-term of nutritional overload. Wistar rats $(n=30)$ were randomly divided to receive either chow diet (C, 5\% fat) or nutritional overload $(\mathrm{H}$, high-fat diet with $29 \%$ fat plus sugar in the drinking water $-300 \mathrm{~g} / \mathrm{L}$ ) for six weeks. Gene expression of TNF- $\alpha$, IKK $\beta$, NFKB, TLR-4 and adiponectin (ADP) was assessed in three fat deposits by Real Time PCR. Results were presented as median \pm semi-range. Comparison between groups was performed by MannWhitney test.

Results: There was no significant differences in final body weight between two groups $(\mathrm{C}=457 \pm 37$ vs. $\mathrm{H}=463 \pm 46, \mathrm{p}=$ 0.708 ). In contrast, the nutritional overload increased the adiposity index in $\mathrm{H}$ group compared to $\mathrm{C}(\mathrm{C}=4.6 \pm 0.9 \%$ vs $\mathrm{H}=7.7 \pm 1.5 \%$, $\mathrm{p}<0.001)$. The gene expression for inflammation-related genes was: 1) $\mathrm{TNF}-\alpha \mathrm{E}(\mathrm{C}=0.912 \pm 0.59$ vs $\mathrm{H}=1.15 \pm 1.61, \mathrm{p}=0.798)$, TNF- $\alpha$ $\mathrm{V}(\mathrm{C}=0.88 \pm 0.64$ vs $\mathrm{H}=0.68 \pm 0.52, \mathrm{p}=0.09), \mathrm{TNF}-\alpha \mathrm{R}(\mathrm{C}=0.99$ \pm 0.93 vs $\mathrm{H}=0.23 \pm 0.31, \mathrm{p}=0.005), 2) \operatorname{IKK} \beta \mathrm{E}(\mathrm{C}=0.82 \pm 0.60$ vs $\mathrm{H}=0.70 \pm 0.79, \mathrm{p}=0.50), \operatorname{IKK} \beta \mathrm{V}(\mathrm{C}=0.93 \pm 0.81 \mathrm{vs} \mathrm{H}=0.314 \pm$ $0.15, \mathrm{p}=0.005), \operatorname{IKK} \beta \mathrm{R}(\mathrm{C}=1.17 \pm 0.42$ vs $\mathrm{H}=0.62 \pm 0.11, \mathrm{p}=$ $0.161), 3) \mathrm{NF \kappa B} E(\mathrm{C}=1.00 \pm 0.38$ vs $\mathrm{H}=0.74 \pm 0.44, \mathrm{p}=0.21)$,

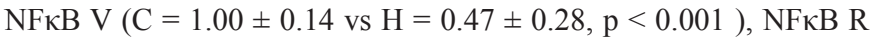
$(\mathrm{C}=0.73 \pm 0.72$ vs $\mathrm{H}=0.50 \pm 0.27, \mathrm{p}=0.03), 4) \mathrm{TLR}-4 \mathrm{E}(\mathrm{C}=0.942$ \pm 0.58 vs $\mathrm{H}=1.02 \pm 0.78, \mathrm{p}=0.75)$, TLR $-4 \mathrm{~V}(\mathrm{C}=1.08 \pm 0.65$ vs $\mathrm{H}=$ $1.00 \pm 0.64, \mathrm{p}=0.80)$, TLR-4 $\mathrm{R}(\mathrm{C}=1.04 \pm 0.72 \mathrm{vs} \mathrm{H}=1.58 \pm 0.90$, $\mathrm{p}=0.03), 5)$ ADP $\mathrm{E}(\mathrm{C}=1.15 \pm 0.61 \mathrm{vs} \mathrm{H}=0.86 \pm 0.55, \mathrm{p}=0.47)$, $\operatorname{ADP} V(\mathrm{C}=0.17 \pm 1.87$ vs $\mathrm{H}=1.16 \pm 2.26, \mathrm{p}=0.23)$, ADP R $(\mathrm{C}=$ $1.17 \pm 0.76$ vs $\mathrm{H}=0.88 \pm 0.43, \mathrm{p}=0.58)$. In conclusion, short term of nutritional overload was able to alter gene expression of some inflammatory mediators in an obesity status but the tissue-dependent manner.

Financial support: FAPESP (14132-0).

\section{P076 \\ Evaluation of Zinc and Vitamin E Supplementation on Bone Metabolism in Diabetic and Osteopenic Rat Model}

Fabricio Melo Santos, Raul Hernandes Bortolin, Francisco Paulo Freire-Neto, João Felipe Bezerra, Marcela Abbott Gal Ururahy, Karla Simone Souza, Melina B. Loureiro, Leandro Vinicius Morais, Anne Caroline S. Barbosa, Yonara M. Oliveira, Heglayne P. Silva, Thamara Rodrigues Melo, Aglauciele M.L. Oliveira, Rosario D.C. Hirata, Mario H. Hirata, Maria G. Almeida, Luciana A.Rezende, Adriana A. Rezende

Federal University of Rio Grande do Norte

Background: Diabetic osteopenia is one of the chronic complications associated to type 1 diabetes. Minerals and antioxidants supplementation plays an important role in bone growth and mineralization.

Objectives: We evaluated the effect of mineral and antioxidant supplementation in diabetic and osteopenic model.

Methods: Diabetes was induced by streptozotocin (STZ - 40 $\mathrm{mg} / \mathrm{Kg}$ of body weight), male Wistar rats were distributed in four groups: Control $(n=5)$, Diabetic $(n=5)$, DI [Diabetic treated with insulin $(n=5)$ ] and DSI [Diabetic supplemented with 2.5 times of calcium and phosphorus, 20 times of vitamin $\mathrm{E}$ and $500 \mathrm{mg} / \mathrm{Kg} / \mathrm{diet}$ of zinc and treated with insulin $(\mathrm{n}=5)]$ and evaluated at 60 days following the induction of diabetes by histological, biochemical and molecular analysis.

Results: Ionized calcium was reduced in the Diabetic, DI and DSI groups compared with Control group ( $<<0.032$, $p<0.0001$, $p<$ 0.0001 , respectively) and no differences in the phosphorus concentrations was observed. The serum alkaline phosphatase (ALP) activity was increased in Diabetic group $(p=0.0007)$, besides an increased Tb.Sp. $(p<0.001)$ and decreased Tb.Th. $(p=0.0065)$, compared to Control group. The possible protective effect of supplementation and anabolic effect of insulin was observed in the reduction of ALP activity and Tb.Sp. in DI ( $p=0.0203, p<0.001$, respectively) and DSI groups $(\mathrm{p}=0.0108, \mathrm{p}<0.001$, respectively) compared to Diabetic group, whereas Tb.Th and BV/TV were increased in the DI $(\mathrm{p}=$ $0.0065, p=0.0006$ respectively) and DSI $(p=0.0088, p=0.0264$ respectively) groups compared to Diabetic group. A reduction of mRNA expression of RANK and $O P G$ was observed in DI and DSI groups when compared with Diabetic group $(\mathrm{p}<0.0001)$, whereas for $R A N K L$ no difference was observed. It was observed also a positive correlation between glucose concentration with ionized calcium $(\mathrm{r}=$ $0.665, \mathrm{p}=0.001), \operatorname{RANK}(\mathrm{r}=0.721, \mathrm{p}<0.001), O P G(\mathrm{r}=0.415, \mathrm{p}=$ $0.049)$ and Tb.Sp. $(\mathrm{r}=0.735, \mathrm{p}<0,001)$ and a negative correlation between glucose concentration with BV/TV $(r=-0.529, \mathrm{p}=0.017)$ supporting the bone loss associated to diabetes.

Conclusions: The downregulation of $O P G$ mRNA expression associated to increased bone volume and protection against bone loss suggest the possible anti-resorptive effect of supplementation on bone besides the insulin therapy. 
P077

\section{Antioxidant Properties of Yerba Mate (Ilex Paraguariensis St. Hil.) Extracts and Its Ability to Chelate $\mathbf{F e}^{2+}$}

Felipa Elzira Melgarecho Bassante, Ana Ceolin Colpo, Maria Eduarda Lima, Hemerson Silva Rosa,

Vanderlei Folmer

Universidade Federal do Pampa-UNIPAMPA

Introduction: Mate tea or simply "mate" is a traditional beverage prepared as infusions of the dried and minced leaves and twigs of the native South America Ilex paraguariensis (St. Hill), Aquifoliaceae (yerba mate). Recently, researchers had demonstrated a high antioxidant activity of aqueous extracts of plant.

Objectives: This study aimed to evaluate and compare the antioxidant properties of three yerba mate samples (El Chasqui, Romance and Yemaipe) that are consumed in Argentine.

Materials and Methods: The herbal compounds were bought from a commercial establishment in Argentina. Aqueous extracts were obtained mimicking the "mate" using a medium size gourd, yerba mate, "mate pump", Kitasato flask and a vacuum pump and extracts of $1^{\circ}, 2^{\circ}, 5^{\circ}, 10^{\circ}, 15^{\circ}$ infusions (mates) were collected for analysis. The scavenging activity was determined by the DPPH assay, nitric oxide-scavenging activity, $\mathrm{Fe}^{2+}$ chelating ability and TBARS based on egg yolk assay system. Statistical analysis was done using analysis of variance ANOVA two-way followed by Tukey's test, $\mathrm{p} \leq$ 0.05 were considered as significant.

Results: Results obtained showed excellent ability of the Ilex paraguariensis extract to scavenge free radicals and reactive intermediates. Was found that all samples showed a significant inhibition of DPPH radical, but the yerba mate El chasqui demonstrated the greatest potential for inhibition, into $>70 \%$. To nitric oxide-scavenging activity and $\mathrm{Fe}^{2+}$ chelating ability the $\%$ inhibition was significant for all the mates and samples. The egg yolk TBARS assay showed that all herbs extracts were able to keep levels at baseline malondialdehyde formation and lipid peroxidation.

Conclusions: The three brands of mate analyzed showed an excellent antioxidant and scavenger free radical activity. Only in the DPPH assay significant differences between the brands tested. Also, was detected a tendency to decrease in its potential in mates $10^{\circ}$ and $15^{\circ}$.

\section{P078 \\ Chemoprotective Activity of Grape Juice Concentrate (G8000) in Chemically Induced Oral Carcinogenesis by 4-Nitroquinoline 1-Oxide: Role of Inflammatory Mediators}

Flávia Andressa Pidone Ribeiro, Gustavo Protásio Pacheco de Jesus, Carolina Foot Gomes de Moura, Andrea Pittelli Boiago Gollucke, Daniel Araki Ribeiro

\section{Universidade Federal de São Paulo-UNIFESP}

Background: In recent decades, several beneficial health effects have been attributed to phenolic compounds present in fruits, vegetables, teas and wines. The grape is considered a major source of phenolic compounds when compared to other fruits and vegetables.

Objectives: The objective of this study was to evaluate the chemoprotective potential of grape juice concentrate (G8000TM) rich with polyphenols during rat tongue carcinogenesis induced by 4-nitroquinoline 1-oxide by means of mRNA expression of eNOS, iNOS, TNF-alpha and COX-2.

Methods: A total of 24 male Wistar rats aged 8 weeks of age were used. The animals were distributed into four groups as follows: Group 1 - received G8000TM orally by gavage for eight consecutive weeks only, Group 2 - received 4NQO at $20 \mathrm{ppm}$ dose during 8 weeks in drinking water and treated with G8000TM at a 1\% dose orally by gavage between the 1st and 4th weeks daily, Group 3 received $4 \mathrm{NQO}$ at $20 \mathrm{ppm}$ dose for 8 weeks in drinking water and treated with G8000TM at $1 \%$ dose orally by gavage between the 5 th and 8th weeks daily, Group 4 (control) received 4NQO at 20 ppm dose for 8 weeks in drinking water daily.

Results: The results showed a decrease in gene expression of eNOS, iNOS, TNF-alpha and COX-2, in which statistically significant differences $(\mathrm{p}<0.05)$ were observed in groups 2 and 3 when compared with group 4 (control).

Conclusion: In summary, these results suggest that G8000TM is able to modulate the oral carcinogenesis as a result of the anti-inflammatory potential demonstrated by modulating the expression of TNFalpha, COX-2, iNOS and eNOS. This work was support by CNPq and Capes.

\section{P079 \\ Maternal Food Restriction in Utero Alters GDNF and WNT11 mRNAS in Kidneys During Nephrogenesis}

\author{
Carmen Grigoletti Mir, José Antonio Rocha Gontijo, \\ Flávia Mesquita
}

School of Medical Science-UNICAMP

A maternal poor diet is frequently associated with increased blood pressure and reduced nephron number in adult offspring. Despite the ubiquity of this model there is uncertainty as to when in development these effects manifest. Mesenchymal to epithelial transformation begins to occur from rat embryo day 14 with ureteric branching and mesenchymal transdifferentiation occurring actively at 20th day 
(e20). The objective of this study was to investigate how these transcription aand growth factors are controlling fetal nephrogenesis following maternal food restriction during pregnancy. Sprague Dawley dams were mated and after sperm detected in vaginal smears, they were separated in 2 gropus: CTRL (control, $\mathrm{n}=3$ ) and FR50\% (food restricted - received 15gof food/day, $n=4)$. At e20, dams were anesthetized and foetuses were quickly removed. Fetus were dissected and kidneys removed and placed at RNA later ${ }^{\circledR}$. Total renal tissue RNA was isolated using RNeasy kit (Qiagen). cDNA was synthetizes using TaqMan ${ }^{\circledR} H i g h$ Capacity cDNA trsncription kit (Life Technologies). The analysis of expression of Pax2, WT1, GDNF and Wnt11 was carried out with TaqMan Gene Expression Master Mix (Life Technologies), and ACTB as endogenous control, and performed in the StepOne Plus PCR system (Life Technologies). The mRNA expression obtained for each gene was compared with CTRL group using the comparative quantification method. All relative quantifications were assessed using DataAssist software, using ${ }^{\Delta \Delta} \mathrm{Ct}$ method. Pregnant rats from CTRL group gained more weight than FR50\% group $(114.3 \pm 11.05$ vs. $48.80 \pm 8.11, \mathrm{p}=0.0028)$. Following the mothers pattern, the offspring from FR50\% group was $32 \%$ lighter than from CTRL group $(\mathrm{p}<0.0001)$. Pax2 and WT1 were normally expressed $(0.805$-fold, $\mathrm{P}=0.1706$ and 0.8376 -fold, $\mathrm{P}=0.1706$, respectively) in FR50\% as compared with CRL e20 kidneys. Pax 2 is a transcriptional activator expressed in mesenchyme and ureteric bud and is regulated by WT1. Wnt11 is a transcription factor regulated by Pax 2 and mRNA is increased (1.16382-fold, $\mathrm{P}=0.0004)$ in FR50\% group. GDNF is also upregulated (1.1235-fold, $\mathrm{P}=0.033$ ). Nephrogenesis is softly regulated by different genes and at the e20 these modifications found in some of these genes can be a key to elucidate why maternal food restriction leads a low nephron number that is one of the causes of adult hypertension.

Financial support: FAPESP (2011/23508-4).

\section{P080 \\ Poor Maternal Diet Alters Fetal Kidney miRNAS Related to Renal Development}

\author{
Flavia Mesquita, José Antonio Rocha Gontijo \\ School of Medical Science-UNICAMP
}

The consequences of fetal programming by maternal low protein diet have been investigated deeply and the hypothesis that the kidney is one of the organs most affected is well accepted by worldwide scientists. We are working in the hypothesis that nephrogenesis is impaired because of changes on pattern of microRNAs that control important genes of development. miR-192, miR-194 and miR-215 have been cited by literature as microRNAs that can be found in the kidney during the development, specially because the targets of these microRNAs are important genes for nephrogenesis. The objective of this study is to investigate how specific microRNAs are expressed on offspring kidney during gestation after maternal low protein diet. The microRNAs that were studied are miR-192, miR-194, miR-215. 8 female rats (Sprague-Dawley, 12w/a) were divided in 2 groups: normal protein (NP) and low protein (LP). Rats from LP group received diet with $6 \%$ of casein, while NP group received diet with $17 \%$ of casein. They were mated and at the day 20.5 of gestation, kidneys were harvested from fetus and processed for real-time PCR. miRNA were extracted with miRVana (Ambion) and real-time PCR processed using TaqMan Universal PCR Master Mix (Applied). The method used was ${ }^{\Delta \Lambda} \mathrm{Ct}$, using U87 as endogenous control. Real-time PCR quantification showed that miR-192, miR-194 and miR-215 expression level were significantly down regulated in offspring kidneys at the end of gestational period. The activin receptor type 2 is a target of these miRNAs and develops a crucial step on the activation of activin, protein of TGF $\beta$ pathway that controls the ureteric branching. We conclude that the fetal programming of adult kidney chronic disease through undernutrition begins with modifications on miRNAs patterns in the fetal stage.

Financial support: FAPESP (2010/05169-5).

\section{P081 \\ Taurine Supplementation Effects on Homocysteine Metabolism and Oxidative Stress in Rats Fed Choline-Deficient Diet}

\author{
Flávia Troncon Rosa, Lucas Vieira Francisco, \\ Thama Borges, Lilian E.C.M. Silva, Alceu Afonso Jordão, \\ Rafael Deminice \\ University of São Paulo
}

Background: Choline-deficient diet impairs methionine metabolism what may increase homocysteine (Hcy) and lipid peroxidation. Taurine is a S-containing amino acid and studies have shown that taurine supplementation can modulate Hcy levels. Also, recent data have shown antioxidant capacity of taurine. However, the effects of taurine supplementation on CDD have been poorly explored.

Objectives: To examine the effects of taurine supplementation on Hcy metabolism in rats fed choline-deficient diet.

Methods: Twenty four rats were divided into 3 groups of 8 rats each: control diet (C), choline-deficient diet (CDD), choline-deficient diet supplemented with taurine (CDDT). The CDD diet was AIN-93 without the choline recommended content. Taurine was supplemented by the inclusion of $2.5 \%$ in the diet. The rats received the diets for 4 weeks.

Results: CDD diet significantly impaired methionine metabolism as evident in increased plasma Hcy (50\%) and decreased hepatic $\mathrm{S}$-adenosylmethionine (25\%) and SAM/SAH ratio (44\%). It was also found decreased liver Bhmt and Gnmt mRNA abundance compared to control. In addition, increased plasma MDA (24\%) and ALT (30\%) and decreased GSH (16\%) and GSH/GSSG ratio (37\%) were also found in the liver of CDD rats compared to control. Surprisingly, taurine supplementation did not prevent any of these perturbations. Taurine supplementation returned Bhmt and Gnmt gene expression to control level. However, it was not enough to prevent increased Hcy concentration and oxidative stress characterized by elevated MDA and decreased GSH and GSH/GSSG ratio. It was not found changes in methionine and cysteine plasma levels or CBS gene expression.

Conclusions: Taurine supplementation did not prevent impaired methionine metabolism and toxicity in rats fed choline deficient diet.

Financial support: PRODOC/CAPES - Brazil. 


\section{P082}

\section{Baccharis Trimera Extract Increases Oxidative Stress Resistance and Glutathione S-Transferase Expression in Caenorhabditis Elegans}

Franciny Aparecida Paiva, Riva de Paula Oliveira, Maria Lúcia Pedrosa, Joyce Costa Guerra

Universidade Federal de Ouro Preto

Carqueja (Baccharis trimera) is a plant widely used in folk medicine as a tea and to treat gastrointestinal diseases and inflammatory processes. The aqueous and hydroalcoholic extracts are rich in antioxidants, such as, esters of quinic acid and flavonoids nepetin, quercetin and isoquercetin. Here we use Caenorhabditis elegans as a model organism to investigate the effects of carqueja hydroalcoholic extract (CHE) on oxidative stress resistance and longevity. First, we determined the CHE capacity to neutralize the DPPH radical as indicative of its capacity to neutralize free radicals. We observed that $\mathrm{CHE}$ at $0.5 \mathrm{mg} / \mathrm{ml}$ inhibited only $19.95 \%$ of DPPH radicals while 5 and 50 $\mathrm{mg} / \mathrm{ml}$ of $\mathrm{CHE}$ quenched $74.27 \%$ and $82.86 \%$ of DPPH radicals respectively. The DPPH scavenging activity of CHE at 5 and $50 \mathrm{mg} /$ $\mathrm{ml}$ did not alter when it was mixed neither with Escherichia coli, the food source for C. elegans, or E. coli killed with the antibiotic kanamycin. To examine the effects of CHE on oxidative stress resistance, wild-type animals were treated for $48 \mathrm{~h}$ in growth medium seeded with E. coli ressupended with either $0.5,5$ or $50 \mathrm{mg} / \mathrm{ml}$ of CHE and then transferred to $7.5 \mathrm{mM}$ of t-butyl hydroperoxide (t-booh) solution. Only animals treated with $50 \mathrm{mg} / \mathrm{ml}$ of CHE showed increased survival after 12 hours exposure to $7.5 \mathrm{mM}$ t-booh. To exclude nonspecific effects from bacterial metabolization, survival on stress conditions were repeated using $E$. coli killed with kanamycin. In these experiments, the oxidative stress resistance induced by $50 \mathrm{mg} / \mathrm{ml}$ of CHE was abolished indicating that bacteria metabolism increases CHE antioxidant power without interfering with its DPPH scavenging activity. To examine the effects of CHE on lifespan, we used the C. elegans strain, fem-1(hcl7), which is a temperature-sensitive mutant which develops into sterile adults at $25{ }^{\circ} \mathrm{C}$ and has lifespan similar to wild-type animals. There was no statistical difference in the longevity between control and treated animals with any of the three concentrations tested. We also examined the expression of two reporter genes encoding Phase II detoxification enzymes: superoxide dismutase $(\operatorname{sod}-3: \because G F P)$ and glutathione S-transferase (gst-4::GFP). We observed that the expression of gst-4::GFP, but not of sod$3: \because G F P$, in animals treated with CHE were significantly increased. These results suggest that $\mathrm{CHE}$ treatment increases $\mathrm{C}$. elegans oxidative stress resistance and that activation of gst-4 might be involved in this protection.

\section{P083 \\ Effects of Oleic and Linoleic Acids in Keratinocytes}

Gilson Masahiro Murata, Renata Gorjão, Rui Curi, Elaine Hatanaka

Universidade de São Paulo

Introduction: Wound healing depends on the balance of restitution, proliferation, and differentiation of epithelial cells such as keratinocytes adjacent to the wounded area. We recently demonstrated that the topical treatment of wounds with oleic and linoleic acids hastens the healing process, however, the mechanisms of action remain unclear.

Aims: Our purpose was to investigate the effect of oleic and linoleic acids on HaCaT cells. We focused on assays that reveal the effect of these fatty acids on the proliferation, loss of membrane integrity and DNA fragmentation of $\mathrm{HaCaT}$ cells. We also analyzed the release of TNF-alpha, IL-1beta and IL- 8 by cells treated with fatty acids and the signaling pathways involved.

Methods: Cells were cultured in the absence and presence of oleic acid or linoleic acid $(10-400 \mathrm{uM})$ to evaluate: (a) toxicity by clonogenic assay, (b) apoptosis/necrosis by flow cytometry, (c) activating of signaling pathways by Western Blot and, (d) the release of cytokines by ELISA.

Results: Levels above $150 \mathrm{uM}$ and $100 \mathrm{uM}$ of oleic and linoleic acids, respectively, significantly reduce the number of colony-forming units with toxic effects. These results were confirmed by cell viability and DNA fragmentation assays by flow cytometry. Nontoxic $(20 \mathrm{uM})$ and toxic $(200 \mathrm{uM})$ doses of oleic and linoleic acids increased the phosphorylation of ERK1/2 and Akt proteins. The release of interleukin- 8 and interleukin- 6 was found to be dose-dependent.

Conclusions: Oleic and linoleic acids can modulate the proliferation and death of keratinocytes. This study was supported by FAPESP (2008/50310-5), CNPq (475841/2011-5 and 306041/2011).

Financial support: CAPES (No 23038.039417/2008-04).

\section{P084 \\ Fish Oil Decreases COX-2, PPARgamma and RELA Gene Expression in Walker 256 Tumor- Bearing Rats}

Gina Borghetti, Ricardo Yamazaki, Adriana Yamaguchi, Isabela Coelho, Danielle Pequito, Sandro Bonatto, Julia Aikawa, Gleisson Brito, Luiz Claudio Fernandes

UFPR

Background: Epidemiological and experimental evidences have shown the pharmacological potential and the nutritional value of diets enriched in fish oil, a high n-3 polyunsaturated fatty acid (n-3 PUFA) content. Alterations in the expression of genes related with inflammation could be an important mechanism whereas n-3 PUFA applies its beneficial effects in pathological conditions, such as cancer.

Objectives: The objective of this study was to investigate the effect of diet supplemented with fish oil in cyclooxygenases 2 (COX- 
2), peroxisome proliferator-activated receptor gamma (PPAR $\gamma)$ and v-rel reticuloendotheliosis viral oncogene homolog A (RelA) gene expression in Walker 256 tumor-bearing rats.

Methods: Male Wistar rats at 70 days of age were fed with regular chow (control W) and supplemented with fish oil (WFO, long chain n-3 PUFAs: eicosapentaenoic acid (EPA) and docosahexaenoic acid (DHA)), (1 $\mathrm{g} / \mathrm{kg}$ body weight) until they reached 100 days of age. Then, they were inoculated with a suspension of Walker 256 ascitic tumor cells $(3 \times 107 \mathrm{~mL})$ and after 14 days they were orthothanized. Total RNA was isolated from tumor tissues and the relative mRNA expression was measured by quantitative real-time reverse transcription (qRT-PCR) to compare the relative expression, using the 2deltadelta CT method.

Results: Fish oil supplementation resulted in a significant decrease in mRNA expression of COX-2 (5.06 \pm 1.10 fold), PPAR $\gamma$ $(2.70 \pm 0.39$ fold $)$ and RelA $(1.21 \pm 0.07)$ in tumor tissue when compared with control group W $(\mathrm{p}<0.05)$.

Conclusions: These results demonstrate the decrease of COX-2, PPAR $\gamma$ and RelA gene expression in tumor tissue in Walker 256 tumor-bearing rats by fish oil. Despite the molecular mechanisms involved in mRNA regulation, are not fully understood, the data suggests that n-3 PUFA may exert their effects via their capacity to attenuate the expression of inflammatory genes, associated in carcinogenisis.

\section{P085}

\section{Nutritional Genomics in Chronic Diseases}

Gisele Oliveira Guaita, Laylla Marques Coelho FEPAR/UFPR

The completion of the Human Genome Project has enabled the development of a new era in the field of nutrition, nutritional genomics. It studies the interactions between nutrition and the genome, including both Nutrigenomics, an area that studies the effects of nutrition on gene expression and function, and nutrigenetics, that studies the effects of genetic variation in response to diet. Food is one of the factors that influence the functioning of the genome, nutritional genomics will then appear in order to understand how diet interacts with the human genome to influence health and disease and, moreover, how genetic variability influences response to diet. The objective of this study was to demonstrate through a literature review, the main electronic databases, the development of nutritional genomics and its progress to the present day, emphasizing the main studies carried out in relation to chronic diseases. Many studies confirm the existence of an association of nutrient intake with the incidence and severity of chronic diseases, so the union of nutrition and genetics can bring great benefits to using the most appropriate interventions and customized to each patient. This work was performed by means of literature, scientific PubMed and Scielo, during the period 2000 to 2012, using the following keywords: Nutritional Genomics, Nutrigenomics, Nutrigenetics, Chronic diseases, Cancer, Obesity, Diabetes Mellitus type 2. Data obtained suggests that carbohydrates, lipids, vitamins and minerals involved in the regulation of gene expression. It is very important to understand the nutrient gene interactions, because the substances present in food can modulate gene expression by modifying the phenotype. The studies reviewed demonstrate the important role of nutritional genomics associated with chronic diseases and confirm the existence of interaction between genes and nutrients, indicating the need for new studies to assess the influence of different nutrients on the effects related to the presence of genetic polymorphisms. With the data set expected to arise in this field, it will be possible in future, a dietary intervention tailored to each individual's genetic profile, which will enable a major improvement in quality of life and prevention of chronic diseases.

\section{P086 \\ Antitumor Activity of Morinda Citrifolia L. (Noni)}

Graciana Freitas Palioto, Juliana Ceccon Voll, Fabio Santos Barros, Tiago Henrique dos Santos Garbim, Suelen Mendes Leles, Priscila da Silva Fraire, Alessandra Paim Berti, Carmem Lucia M.S.C. Rocha

Universidade Estadual de Maringá

Noni (Morinda citrifolia L.) has been consumed for thousands of years by Polynesians, because of its nutritional and therapeutic components. Many studies have reported that fruits, leaves and roots of this plant have been used to prevent and cure several diseases due to nutritional and therapeutic values it has. The fruit juice is the main way noni is marketed in many countries around the world and it is growing. Among the biological activities related to noni, we can mention the antiviral, analgesic, hypotensive, immune modulation, antioxidant, hypoglycemic and anti-inflammatory activity. However, noni is mainly studied due to its antitumor activity. Studies have demonstrated the efficacy of noni in lung cancer, Lewis carcinoma, liver cancer, colorectal cancer and breast cancer. The antitumor action was demonstrated through several ways: by stimulation of immune system, production of T-cells, thymocytes and macrophages that are important mediators for cytotoxicity and blockage of tumor growth, inhibitory activity for Ras protein, inhibition of protein AP-1 transactivation, preventing the formation of DMBA-DNA adducts, induction of apoptosis in tumor cells and decreasing the proliferation rate of tumor cells. Other studies were conducted with the combination of noni and chemotherapeutics, the results indicated that noni can be considered an adjunct in the treatment of some cancers such as breast cancer. It was also found an increased survival time of animals and reduction of DNA damage caused by the chemotherapeutic. There are about 200 publications in indexed journals on international databases in the last five years, which demonstrates the great potential of the study and application of this plant on human health, with great emphasis on nutrigenomics. 


\section{P087}

Effect of the Noni Juice (Morinda Citrifolia Linn) on the Genotoxicity and Morphophysiological Aspects of Alloxan Induced Diabetes in Rats

\section{Juliana Ceccon Voll, Fabiane Apareci Vilar Mendes, Graciana Freitas Palioto, Cecilia Edna Mareze da Costa, Carmem Lucia M.S.C. Rocha \\ Universidade Estadual de Maringá}

Hypoglycemic agents and natural antioxidants are important adjuncts in the treatment of the diabetes mellitus (DM), because they can mitigate the harmful effects of the excessive production of free radicals and reactive species of oxygen, associated to the oxidative stress. The latter has an important role in the pathogenesis of the type $1 \mathrm{DM}$ and its complications. Studies have shown that noni (Morinda citrifolia Linn), a potential source of nutrients and antioxidants, can be complementary in the treatment of the complications of DM. In this work we utilized an experimental diabetes model induced by alloxan in order to evaluate the effects of the noni juice on genotoxic, physiological and morphological parameters of diabetic rats. DNA damage in diabetic rats, as well as the effects of the noni juice on the recovery of these damages, was evaluated with the alkaline comet assay. It were assessed the effects of noni juice on morphological aspects of pancreatic islet, hyperglycemia and plasmatic levels of aspartate aminotransferase (AST) and alanine aminotransferase (ALT). Male Wistar rats with 50 days of age were divided into four groups (each group with $\mathrm{N}=10$ ): diabetic treated with noni (DT), diabetic control (DC), normal treated with noni (NT) and normal control (NC). A pre-post treatment was realized with noni juice at a dose of $0.50 \mathrm{~mL} / \mathrm{kg}$ by a period of ten days before the induction of DM by alloxan and ten days after the induction. The results of the comet assay indicated genotoxic potential of the type 1 diabetes in alloxanized rats. The noni juice decreased the damage index, improved the morphological aspects of the pancreatic islets, reduced the hyperglycemia and also reduced the plasmatic levels of AST and ALT in diabetic rats.

\section{P088 \\ Toxic and Genotoxic Effect of Cafeteria Diet in Wistar Rats and Its Reversion by Rat Chow Diet and Noni Supplementation (Morinda Citrifolia)}

\author{
Gabriela Nunes Marsiglio Libra, \\ Cecilia Edna Mareze da Costa, Graciana Freitas Palioto, \\ Ana Cláudia Munhoz, Rodrigo Factori, Bruno Carabelli, \\ Carmem Lucia M.S.C. Rocha \\ Universidade Estadual de Maringá
}

Toxicologic genetics search the damage on DNA which can be caused by food additives. The cafeteria food is rich in lipides and salt and poor in fibers. Because of this composition plus the high quantity of synthetic aditives, this type of food is characterized as carcino- genic diet. Many researchers have been made with nutraceuticals and phytotherapicals, showing their capability of genome protection. The fruit juice of noni has been studied because it's medicinal and nutritional properties. One important and known activity of this juice is the antitumoral and antigenotoxic property. The work studied the possibility of reversion of the toxicity with a change of the diet to a balanced diet supplemented or no with noni juice. The analysis was made by physiologic parameters and comet test for genotoxicity. Wistar rats male and female, received controlled diet since 21 days, for 23 weeks. The experiment was divided into two steps. First, the rats were divided in two groups, one receiving control alimentation (rat chow control - RAco) and other receiving cafeteria diet (cafeteria control - CAco), for 20 weeks. These rats were analyzed for physiologic parameters once a week and by comet assay one time each two weeks. The second step was developed during the following 50 days. At this time, the previous 2 groups were subdivided into other, each with 4 animals: rat chow control (RAco), rat chow noni (RAno), cafeteria control (CAco), cafeteria noni (CAno), cafeteria-rat chow control (CRco) e cafeteria-rat chow noni (CRno). The cafeteria-rat chow groups during these three weeks were feed with rat chow and the noni groups where supplemented with noni juice $(0.50 \mathrm{~mL} / \mathrm{Kg})$ by esophagic catheter daily. The cafeteria diet led to obesity by hypertrophy and hiperplasy of adipose tissue. There were decrease on glucose tolerance and insulin sensitivity and increase on jejune glicemic. It was detected reduction on lecocites number, HDL and AST. All these parameters were changed to normal levels when rats were submitted to rat chow diet. The comet test results had shown that cafeteria diet presents genotoxic effects and female was more susceptible to genotoxicity. When animals of cafeteria group had change their diet to rat chow, the comet damage parameters ID and FD were decreased, showing reversion. The noni juice had decreased genotoxicity in all diet groups: rat chow, cafeteria, and cafeteria- rat chow, showing antigenotoxic potential.

\section{P089 \\ Proteomics Analysis of the Bone Marrow Extracellular Matrix of Mice Submitted to Protein Malnutrition}

Graziela Batista Silva, Jackeline Oliveira S. Beltran,
lana Suly S. Katz, Layra Lucy Albuquerque,
Orlando Garcia Ribeiro, Primavera Borelli

Faculty of Pharmaceutical Sciences-USP

Introduction and Objectives: Hematopoiesis is a dynamic process that is modulated by various factors as the extracellular matrix $(E C M)$ that provides a support structure and important role in many functions for hematopoietic cells such as differentiation, proliferation, apoptosis and migration. Mice subjected to protein malnutrition show changes in the cell cycle of hematopoietic stem/progenitor cell, resulting in a hypoplasia marrow leading to anemia and leukopenia. The aim of this study is to evaluate possible changes in bone marrow of ECM, established through long-term cultures of bone marrow cells (LTBMC), by analyzing two-dimensional gel.

Methods: Male mice C57BI/6J, adults, were divided into two groups. The control group $(\mathrm{C})$ received a diet with $12 \%$ casein and the 
malnourished group (M) with $2 \%$ casein for 5 weeks. After this period were collected bone marrow cells to LTBMC for $28^{\text {th }}$ and $35^{\text {th }}$ days then was performed protein extraction for two-dimensional gel analysis.

Results and Conclusions: In the analysis of two-dimensional gel of LTBMC in both groups we select regions where there were some intensity changes. We observed a difference in the proteomic profile between groups and days analyzed. LTBMC M group in $28^{\text {th }}$ days failed or decreased production proteins that are produces the $\mathrm{C}$ group. In $35^{\text {th }}$ days of LTBMC the $\mathrm{M}$ group maintains a profile of some proteins found in $28^{\text {th }}$ days, but produced proteins that $\mathrm{C}$ group did not produce. This proteomic profile difference between the groups suggests a remodeling of ECM that leads to changes in the cell cycle of hematopoietic stem/progenitor cell thus resulting in bone marrow hypoplasia. The sequencing these proteins will help to elucidate the involvement of ECM in the maintenance of hematopoiesis.

Financial support: FAPESP (09/53902-6).

P090

\section{Anti-Inflammatory Effects of Omega-3 Fatty Acids on Colorectal Carcinogenesis Occurs Through Lipid Sensor GPR-120 in DIO Mice}

\author{
Gustavo Duarte Pimentel, Guilherme Z. Rocha, \\ Juliana Alves Camargo, Thayana Oliveira Micheletti, \\ Maria Carolina Mendes, Carlos K. Katashima, \\ Vagner Rodrigues Silva, Eduardo Rochette Ropelle, \\ Mário Abdalla Saad, José Barreto Carvalheira \\ Department of Internal Medicine, UNICAMP-Brazil
}

Rationale: Obesity-associated inflammation is associated with an increased risk of several types of cancer, such as the colon cancer. However, the polyunsaturated fatty acid, Omega-3 has anti-neoplastic effects. Thus, we sought to determine the effects of Omega-3 on colorectal carcinogenesis in diet-induced obesity (DIO) mice.

Methods: Four-wk-old male mice (C57BL6/J) were placed in high-fat diet (DIO group) $(\mathrm{n}=6)$ or high-fat diet supplemented with $30 \% \mathrm{kcal}$ from fat with omega-3 (ROPUFA 75'®) (Omega-3 group) $(\mathrm{n}=5)$. After one wk of diet, the mice were injected i.p. with $12.5 \mathrm{mg} /$ $\mathrm{kg}$ azoxymethane. After 5 days, 2\% DSS was given in the drinking water for $5 \mathrm{~d}$, followed by $14 \mathrm{~d}$ of regular water. This cycle was repeated twice and mice were sacrificed eight days after the last cycle. Colon and tumour tissues were removed for immunobloting and serum for cytokines analysis by ELISA. O2 consumption and $\mathrm{CO} 2$ production were measured through of Gas Analyzer (Panlab $\mathbb{R}$ ). Statistical analysis: Student's test t.

Results: We found that Omega-3 group non-significantly reduced body weight $(20.7 \pm 2.7$ vs. $24.8 \pm 1.3 \mathrm{~g}, \mathrm{p}=0.10)$ and adipose tissue weight $(0.36 \pm 0.03$ vs. $0.88 \pm 0.06 \mathrm{~g} / 100 \mathrm{~g}$ b.w., $\mathrm{p}=0.09)$ compared to DIO group. However, no change was found in $\mathrm{O} 2$ consumption and $\mathrm{CO} 2$ production. Tumour size and number were reduced in the Omega- 3 group $(2.4 \pm 0.5$ vs. $3.2 \pm 0.2 \mathrm{~mm}, \mathrm{p}=0.049)$ and $(3.5 \pm 0.7$ vs. $8.0 \pm 1.0$ units, $p=0.006)$, respectively. Serum analysis showed no significant lessening of TNF- $\alpha$ in Omega-3 group $(13.0 \pm 4.4$ vs. $26.0 \pm 15.0 \mathrm{pg} / \mathrm{mL}, \mathrm{p}=0.09)$, with similar values of IL-6 and IL-10. In the other hand, Western blot analysis revealed that
Omega-3 reduced IKK and JNK phosphorylation in both tumour and colon tissues. It was accompanied by the reduction of TNF- $\alpha$ expression in the tumor tissue and IL- $1 \beta$ and INOS expression in the colon of DIO mice. Furthermore, we sought to investigate as the omega-3 could to reduce the inflammatory process and interestingly was observed the of omega-3 fatty acids acts as a ligant to G-coupling protein receptor (GPR120) and it receptor is associated with $\beta$-arrestin protein reducing the anti-inflammatory markers.

Conclusions: Omega-3 promotes anti-inflammatory effects, reduction of size and number of tumor in colorectal cancer probably through of GPR120.

Disclosure of Interest: None Declared.

Financial support: FAPESP (2011/02806-7).

\section{P091 \\ Genetic Risk Score as a Predictor of Weight Loss During a Lifestyle Intervention in Obese Adults}

Harry Freitag Luglio, Erik Aller, Freek Bowman, Edwin Mariman, Marleen Van Baak

Maastricht University

Background: Studies showed that certain Single Nucleotide Polymorphisms (SNPs) can increase the risk of obesity. However the role of SNPs in weight loss of overweight and obese individual during a lifestyle intervention is still unclear. This might due to weak associations between these SNPs and their phenotype.

Objectives: The aim of this study was to investigate genetic predisposition as predictor of weight changes during a lifestyle intervention program provided by an obesity treatment centre, both by single SNP analysis and by compilation of genetic risk score (GRS) using multiple SNPs.

Methods: This is an observational study in 554 clients of the obesity treatment centre CO-EUR (Centre for Obesity Europe, Heerlen, The Netherlands) who followed a lifestyle intervention program for a minimum of 6 months. Seven SNPs, ADRB2 (rs1042713), FTO (rs9939609), PPARg (rs1801282), Near GNPDA2 (rs10938397), LEP (rs4731426), PLIN6 (rs1052700) and CNTF (rs1800169), were chosen based on previous reports, which showed their association with weight or BMI changes (loss or regain) during an intervention program. For each of the 7 SNPs we evaluated the differences in weight and changes in BMI after 3 months and 6 months of intervention. For each subject a GRS was calculated from the number of risk alleles of the 7 SNPs.

Results: No SNPs were related to changes in the first 3 months. The A allele of ADRB2 was related to BMI loss in the first 6 months (allele effect size $=0.54$, precessive $=0.0004$ ), but not to weight loss (allele effect size $=0.95, \mathrm{p}>0.05$ ). The $\mathrm{C}$ allele of PPARg was related to weight loss in the first 6 months (allele effect size $=0.62$, pdominant $=0.02$ ), but not to BMI loss (allele effect size $=0.31, \mathrm{p}>0.05$ ). The SNP's in FTO, Near GNPDA2, LEP, PLIN6 and CNTF were not related to BMI and weight loss after the first 6 months of intervention ( $p>0.05$ for all). A cut-off point of 10 was used based on the mean value of the GRS. After 6 months of lifestyle intervention, subjects with 10 or more GRS points lost more weight than those who had less 
than 10 GRS points (mean differences $=1.65 \pm 0.82 \mathrm{~kg}, \mathrm{p}=0.045$ ). The differences were not seen after the first 3 months of intervention.

Conclusions: GRS based on these 7 SNPs is a potential predictor of 6-month weight loss during a lifestyle intervention program. Further research is needed to search for the optimal composition of SNPs for the prediction of weight loss.

\section{P092 \\ Effects of Fruit Juice Intake on Inflammatory and Oxidative Stress Related Promoter Genes}

Raquel Cristina L. Assis, Helen Hermana M. Hermsdorff, Josefina Bressan

Federal University of Viçosa

A low-chronic inflammatory status is a recognized link between excessive adiposity and metabolic syndrome features and atherosclerosis. In fact, an increased expression of nuclear-factor-kappa-B $(\mathrm{NF} \kappa \mathrm{B})$, a redox-sensitive and proinflammatory transcription factor, has been found jointly with increased expression of cytokines, adhesion molecules, and oxidative stress markers in obese individuals. In turn, high-fruit diets have resulted in protective effects against metabolic and cardiovascular diseases. Polyphenols from this food-group have been largely responsible for their antiatherogenic activities, mainly through modulating expression of genes encoding antiinflammatory and antioxidant proteins. Thus, we performed a literature review to identify the current evidence about the effect of fruit juice intake in expression of genes involved in inflammatory response and oxidative stress. We identified publications from electronic databases. Key-words used were: "juice" (included in title/abstract) and/ or "inflammation", inflammatory", "gene expression", "oxidative stress". The titles and abstracts of all identified studies by the electronic searches were screened, and full texts of potentially relevant studies were evaluated for inclusion. Review articles, editorials, nonEnglish articles, articles which described in vitro effects of fruit compounds, juice fruit effects on not inflammatory or oxidative stress related promoter genes were excluded. Overall, 18 studies accessing effects of fruit juice intake in gene expression/concentrations of inflammatory and oxidative stress markers in vivo were included. The researched juices were bilberry $(n=4)$, pomegranate $(n=4)$, açaí $(n=1)$, apple $(n=2)$, and orange juice $(n=7)$. Bilberry juice was associated with significant decreased concentrations of interleukins- 6 and 15: all target genes for $\mathrm{NF \kappa B}$. Intake of pomegranate and açaí juices were associated with reduction of lipid oxidation. Expressions of antioxidant enzymes genes (glutathione reductase, glutathione peroxidase) were up-regulated by açaí and apple juices. Orange juice consumption induced changes in expression of 3,422 genes and even neutralized the proinflammatory effect of a high-fat meal. In conclusion, this review suggests habitual fruit juice consumption may promote a beneficial modulation of inflammatory and oxidative stress related promoter genes.

Acknowledgments: To the CAPES and the FAPEMIG by research grant and financial support, respectively.

Financial support: CAPES/ FAPEMIG.

\section{P093 \\ Indicated for the Young Investigator Award Methylation of IL6 Gene Promoter in Peripheral White Blood Cells Is Associated with Truncal Fat and Plasma Free Fatty Acids in Young Women}

\author{
Helen Hermana M. Hermsdorff, Fermín I. Milagro, \\ Javier Campión, M. Ángeles Zulet, J. Alfredo Martínez \\ Federal University of Viçosa/University of Navarra
}

Background: Increased serum and gene expression levels of interleukin-6 (IL6) have been associated with obesity and metabolic disorders. However, no studies have analyzed the methylation status of IL6 promoter in relation to these pathologies.

Objectives: We assessed the cytosine methylation levels of IL6 gene promoter in peripheral white blood cells (WBC) of young women, and its relation with central adiposity (truncal fat - TF\%) and serum lipid profile.

Methods: A group of 40 healthy young women $(21 \pm 3$ years old) was assigned into two groups according to the median: 20 with low truncal fat (LTF, $<52.3 \%)$ and 20 with high truncal fat $(\mathrm{HTF}, \geq$ $52.3 \%$ ). Anthropometry variables were recorded, while the plasma IL6 concentrations were quantified. DNA from WBC was isolated and cytosine methylation levels of the $\mathrm{CpG}$ sites found in two regions of IL6 gene promoter (called regions 8 and 14) analyzed by Sequenom EpiTyper. In addition, the global $\mathrm{CpG}$ methylation level for each region was calculated by the average of $\mathrm{CpGs}$ values (3 $\mathrm{CpGs}$ sites for region 8 and $7 \mathrm{CpGs}$ for region 14). Truncal fat was estimated by the equation: $[\Sigma$ (subscapular and suprailiac thicknesses)/ $\Sigma$ (triceps, biceps, subscapular, and suprailiac thicknesses)] x 100 .

Results: The women with HTF showed lower global cytosine methylation levels in the region 8 of IL6 gene promoter when were compared to women with LTF $(0.82 \pm 0.04$ vs. $0.85 \pm 0.04, \mathrm{P}=$ $0.032)$, while there was no difference $(0.30 \pm 0.05$ vs. $0.29 \pm 0.05, \mathrm{P}=$ 0.588 ) in the global methylation levels of the region 14. In addition, global cytosine methylation of the region 8 was negatively correlated with truncal fat $(\mathrm{r}=-0.35, \mathrm{P}=0.028)$ and plasma free-fatty acids $(\mathrm{r}=$ $-0.33, \mathrm{P}=0.037)$. In the linear regression model $(\mathrm{R} 2=0.20, \mathrm{P}=$ $0.012)$, TF \% values were negatively associated with global DNA methylation of region $8(\beta=-0.429, \mathrm{P}=0.011)$, independently from plasma IL6 and free-fatty acids concentrations. There was no significant correlation between plasma IL6 and methylation of any GpG of IL6 gene promoter.

Conclusions: Women with higher truncal fat and higher plasma free-fatty acids showed lower methylation levels in region 8 of IL6 promoter in WBC. However, further studies are needed to understand the intrinsic mechanisms and its importance in the regulation of IL6 secretion and functions.

Funding: Línea Especial about Nutrition, Obesity and Health (University of Navarra LE/97) and FAPEMIG (Brazil). 
P094

\section{Fibrates and Fish Oil, But Not Corn Oil, Upregulate the Expression of Cholesteryl Ester Transfer Protein (CETP) Gene}

Helena Fonseca Raposo, Patrícia Riva Patrício, Mariana Costa Simões, Helena Coutinho F. Oliveira

Depto Biologia Estrutural e Funcional, UNICAMP

Background: CETP acts in the reverse cholesterol transport mediating the transfer of esterified cholesterol from HDL, in exchange for triglycerides, to other lipoproteins that deliver this cholesterol to the liver. In this way, CETP reduces plasma HDL-cholesterol and may increase atherosclerosis risk. Fibrates and fish oil are used to treat hypertriglyceridemia and their actions are mediated by the activation of the nuclear receptors named PPAR $\alpha$. n-3 and n- 6 polyunsaturated fatty acid found in fish and corn oils, respectively, are natural ligands whereas fibrates are synthetic ligands for PPAR $\alpha$.

Objectives: To study the effects of PUFA and fibrates on the CETP expression.

Methods: CETP transgenic mice were treated for 2 weeks with 9 $\mathrm{g} / \mathrm{Kg}$ of fish oil (FO, n-3 PUFA rich), corn oil (CO, n-6 PUFA rich) and saline. In addition, hypertriglyceridemic CETP/apo CIII double transgenic mice were treated for 2 weeks with gemfibrozil (GEM, $200 \mathrm{mg} / \mathrm{Kg}$ ), fenofibrate (FENO, $100 \mathrm{mg} / \mathrm{Kg}$ ), bezafibrate (BEZA, $100 \mathrm{mg} / \mathrm{Kg}$ ) and vehicle (control). Levels of plasma lipids, CETP, adipokines and liver CETP mRNA were determined.

Results: Compared to control, all fibrates treatment elevated plasma cholesterol (CHOL) levels and only FENO significantly diminished triglyceridemia (50\%). Elevation of CETP liver mRNA levels and plasma activity were observed in FENO (53\%) and GEM ( $75 \%$ ) groups. Concerning the oil supplemented studies, compared to saline, FO reduced the plasma levels of non-esterified fatty acid (NEFA) (24 to $28 \%$ ), CHOL (15\%) and HDL-CHOL (30\%) levels. $\mathrm{CO}$ treatment had no effect on NEFA and HDL-CHOL, but increased slightly CHOL $(7 \%, p<0.05)$. None of the two oil treatments affected the plasma triglycerides levels. Compared to saline, FO increased adiponectin and reduced leptin plasma levels, whilst CO increased leptin levels. FO, but not CO, significantly increased plasma CETP mass ( $85 \%$ in male and $96 \%$ in female) and activity (26\% in male and $20 \%$ in female) as well as raised liver CETP mRNA (19-36\%).

Conclusions: Fibrates and fish oil, but not corn oil, upregulate CETP expression at both mRNA and protein levels. Since they are known as PPARa ligands, we postulate that there is a functional PPAR responsive element in the CETP promoter region.

Financial support: FAPESP, CNPQ, CAPES.

\section{P095 \\ Cupuaçu Phenolic Compounds Prevented the Development of Glucose Intolerance Associated with a High Fat/High Sucrose Diet}

Helena Rudge de M. Barros, William Festuccia, Franco Lajolo, Rui Curi, Maria Inés Genovese

Faculdade de Ciências Farmacêuticas USP

The incidence of obesity and type 2 diabetes has reached epidemic proportions affecting billion people worldwide. The discovery of innovative ways to prevent and treat these diseases is essential to minimize its impact on population's health and the economy of countries. Many studies have shown that bioactive compounds in foods influence our overall health. Cupuaçu is a native fruit from the Amazon region, with a large number of phytochemicals that can act on body metabolism. Thus, in this study we aimed to investigate the effects of phenolic extracts of cupuaçu in the prevention of insulin resistance and obesity induced by high fat/ high sucrose diet (HF/HS) in mice. A phenolic extract phenolic of cupuaçu purified by solid phase extraction in resin $\mathrm{C} 18$ was administered to mice at 2 different concentrations ( 0.1 or $0.3 \mathrm{mg}$ of gallic acid equivalent/mice/day) by oral daily gavage for a period of 65 days. Mice were evaluated for insulin and glucose tolerance tests, antioxidant capacity, lipid and glucose levels, urea, creatinine, aspartate aminotransferase e alanine aminotransferase. Cupuaçu extracts did not affect body weight gain or mass of several fat depots, skeletal muscle, liver and heart indicating that extracts of cupuaçu were not effective on preventing obesity. Despite of the absence of effects on body weight and adiposity, cupuaçu extract at lower, but not higher dose, completely prevented the development of glucose intolerance associated with HF/HS feeding. In conclusion, phenolic extracts of cupuaçu have preventive effects against the glucose intolerance associated with HF/HS diet, but the mechanisms underlying these actions need to be further investigated.

\section{P096 \\ High-Fat Diet Enriched with Fish Oil Modulates Lymphocyte Intracellular Pathways}

Heloísa Helena Alves, Cesar Miguel Momesso, Jarlei Fiamoncini, Kim Guimarães Caçula,

Maria Fernanda Cury-Boaventura,

Tania Cristina Pithon Curi, Rui Curi,

Sandro Massao Hirabara, Renata Gorjão

Universidade Cruzeiro do Sul

Background: Lymphocyte activation plays an important role in several inflammatory diseases. High-fat diets rich in saturated and n- 6 fatty acids are related to obesity development and immune system dysfunction. 
Objectives: The aim of this study was to compare the effect of diets with different contents of total lipids and n-3 fatty acids on lymphocyte activation in C57BL/6 mice.

Methods: C57/BL6 mice were fed with control diet (chow) or diets containing either fish oil at $4 \%(\mathrm{NF})$ and $40 \%$ (HF) or lard at $4 \%$ $(\mathrm{NL})$ and $40 \%(\mathrm{HL})(\mathrm{wt} / \mathrm{wt})$ for four weeks $(\mathrm{n}=29)$. Afterwards, lymphocytes were isolated from the mesenteric lymph nodes and cell proliferative capacity (BrDU incorporation), percentage of $\mathrm{T}$ regulatory (Treg) cells and phosphatidylserine externalization (PE) were evaluated by flow cytometry (BD FACS Aria II) and analyzed using BD-Diva software. Protein kinase B (Akt), 3-phosphoinositide dependent protein kinase-1 (PDK-1) and protein kinase zeta (PKCzeta) phosphorylation and expression was performed by westernblotting.

Results: Lymphocytes from HL group presented a higher percentage of cells in apoptosis when compared to NL (40.6\% higher), NF $(55.32 \%)$ and HF (36\%) with $\mathrm{p}<0.05$ for all comparison. Concanavalin A stimulated lymphocyte proliferation from NL groups was higher than chow, NF and HF groups $(\mathrm{p}<0.01$ for all comparison). Lymphocyte proliferation from HL group was $73 \%$ higher than HF $(p<0.001)$. HF group presented an increase in the percentage of Treg cells in relation to the other groups. In the same way, PDK1 and Akt phosphorylation was higher in HL group when compared with the others. PDK-1 phosphorylation was higher in NL and HL groups.

Conclusions: High fat diet with low content of omega-3 fatty acids leads to disruption of lymphocyte regulation characterized by a decrease of Treg cells and increase of phosphorylated protein expression related to lymphocyte proliferation. High fat diet enriched with fish oil promoted an increase on the percentage of Treg cells decreasing phosphorylation of proteins related to proliferation.

Financial support: FAPESP (2011/15260-2).

\section{P097 \\ In Vitro Antioxidant Activity and Chromatographic Analysis of Extracts Obtained from Sida Tuberculata R.E. Fries (Malvaceae)}

\author{
Hemerson Silva Rosa, Caroline Silveira Martinez, \\ Vanessa Brum de Camargo, Ana Ceolin Colpo, \\ Maria Eduarda Lima, Felipa Melgarecho Bassante, \\ Felipa Melgarecho Bassante, Vanderlei Folmer, \\ V. Cechinel-Filho, Andreas Loureiro Mendez
}

Universidade Federal do Pampa

Background: Sida tuberculata R.E. Fries. is a herbaceous species extensively found on Pampa Biome region of Rio Grande do Sul, Brazil. Popularly, Sida species are known as "guanxuma", being used in folk medicine for several illnesses like ulcer, abscesses, cardiovascular diseases and urinary disorders.

Aims: The present study proposed the evaluation of extracts from $S$. tuberculata, investigating the in vitro antioxidant activity and the chromatographic profile by HPLC.

Methods: Plant material of $S$. tuberculata was collected in August of 2011 in Uruguaiana (RS, Brazil). The hydroethanolic and aqueous extracts of leaves and roots were obtained by percolation and infusion, respectively. The HPLC analyses were performed in a by acetonitrile and phosphoric acid $0.05 \%, \mathrm{pH} 3.0,0.8 \mathrm{~mL} / \mathrm{min}$ flow rate, and detection by UV system, $340 \mathrm{~nm}$. In vitro antioxidant activity was evaluated using DPPH and TBARS based on egg yolk assay system.

Results: In antioxidant activity assay, the results demonstrated that the smallest significant concentrations for the DPPH radical assay were $0.06,0.3$ and $0.6 \mathrm{mg} / \mathrm{mL}$ for hydroethanolic leaf, hydroethanolic root and aqueous leaf and root extracts, respectively. The hydroethanolic extracts were the most effective in the inhibition of malondialdehyde formation and lipid peroxidation, showed by TBARS assay. According to the chromatographic profiles obtained, the phenolic compounds could be detected on leaves and roots extracts, being chemically related to kaempferol and quercetin glycosides.

Conclusions: The analytical system proposed allowed the investigation of the phenolic composition in the studied material, which can be relation to the great antioxidant activity observed for the hydroethanolic and aqueous extracts.

Financial support: CAPES.

P098
Maternal Methyl Donor Supplementatio
and High-Fat Sucrose Diet During Lacta
Modify Adult Offspring Response to an
Obesogenic Diet
Paúl Cordero, Fermín I. Milagro, Javier Campión,
J. Alfredo Martínez
University of Navarra, Spain

Background: Perinatal maternal nutrition affects the offspring to develop future diseases in adult life. Novel studies have described the preventive effects of methyl donor supplementation on obesityinduced liver fat accumulation in adult rats.

Objectives: The aim was to analyze the response to an obesogenic diet during adulthood depending on maternal nutrition during lactation, by feeding mothers with a high-fat-sucrose (HFS) diet with or without methyl donor supplementation.

Methods: Thirty-six female Wistar rats were fed a control diet during pregnancy. After weaning, mothers were assigned to four dietary groups, chow diet (Control), chow diet supplemented with a methyl donor cocktail (Control supp), HFS diet (HFS) and HFS diet supplemented with a methyl donor cocktail (HFS supp). Supplementation cocktail includes choline, betaine, folic acid and vitamin B12. After lactation, male offspring were transferred to chow diet and at week 12th they were fed a Control or HFS diet during eight weeks. Body weight and fat percentage, tissue weights and fat content, plasma biochemical markers and a liver gene expression profile were determined.

Results: Adult animals whose mothers were fed an obesogenic diet were heavier and with higher length and adiposity content. Maternal supplementation with methyl donors in HFS dams increased offspring's fat depots when fed the HFS diet in adulthood and showed a decrease in liver fat accumulation. Furthermore, Dnmt1, Dnmt3a, Srebf1 and Mif expression levels in liver were altered by the adult 
diet, whereas Lepr, Mif and Dnmt1 mRNA levels showed changes depending on maternal diet and methyl donor supplementation.

Conclusions: The intake of an obesogenic diet during lactation influences offspring development and their dietary response during adulthood. Maternal supplementation with methyl donors could redistribute fat storages preventing fat accumulation in liver. Maternal and adult dietary intakes also impaired hepatic mRNA levels of genes related with obesity and liver metabolism, as well as with epigenetic related genes such as DNA methyltransferases.

\section{P099 \\ Effect of Protein Malnutrition and the Mechanisms of Apoptosis, Necrosis and Autophagy in Marrow Hypoplasia}

\section{Jackeline Soares O. Beltran, Graziela Batista Silva, Dalila Cunha Oliveira, Ed Wilson Santos, Primavera Borelli \\ Faculty of Pharmaceutical Sciences USP}

Introduction and Objectives: Protein restriction alters physiological responses including proliferation and cell death. We have shown histological changes in protein malnutrition and hypoplasia in the bone marrow (BM) and this study evaluated the participation of apoptosis, necrosis and autophagy in hypoplasia in malnutrition.

Materials and Methods: $\mathrm{C} 57 \mathrm{BL} / 6 \mathrm{~J}$ mice were fed a normal diet $(12 \%$ protein, Control group) or low protein $(2 \%$ protein, Malnourished group) for 5 weeks. Apoptosis and necrosis were evaluated by flow cytometry (FC), cells in total BM labeled Annexin V and PI caspase 3, Bcl-2 was measured by Western Blot (WB). Autophagosomes were evaluated by FC and mTOR, p-mTOR, Akt, p-Akt and LC3B-II by WB. Serum samples were assessed by HPLC method, liquid chromatography.

Results: The BM of the group $\mathrm{M}$ presented hypocellular $(\mathrm{C}: 13.98 \pm 1.4 \mathrm{n}=10, \mathrm{M}: 4.72 \pm 0.46, \mathrm{n}=15$ ). There was no difference between groups in the apoptosis and necrosis, but the expression of total mTOR $(\mathrm{C}: 59.23 \pm 2.28 \mathrm{n}=4, \mathrm{M}: 81.98 \pm 3.66 \mathrm{n}=5)$ was significantly higher in group M and p-mTOR (C:72.13 $\pm 11.45 \mathrm{n}=7$, M:34.81 $\pm 9.45 \mathrm{n}=5$ ) was significantly lower in group M. There was no difference in total Akt, but p-Akt(C:63.85 $\pm 4.64 \mathrm{n}=6, \mathrm{M}: 45.42 \pm$ $7.52 \mathrm{n}=6$ ) was significantly lower in group $M$. There was an increase in autophagosomes group $\mathrm{M}(\mathrm{C}: 32.37 \pm 4.71 \mathrm{n}=3$, M: $48.90 \pm 5.02$ $\mathrm{n}=6$ ) and significant increase in expression of LC3B-II in group M $(\mathrm{C}: 45.60 \pm 10.67 \mathrm{n}=6, \mathrm{M}: 74.92 \pm 5.74 \mathrm{n}=5)$. Amino acids: Methionine $(\mathrm{C}: 6.50 \pm 0.46, \mathrm{M}=4.52 \pm 0.30$ ), Valine ( $\mathrm{C}: 17.86 \pm$ 1.05 , M: $11.03 \pm 0.17)$, Taurine (C: $70.50 \pm 9.66, \mathrm{M}: 38.26 \pm 5.86)$, Lysine ( C: $35.05 \pm 1.41, \mathrm{M}: 26.05 \pm 1.4$ ), Isoleucine ( C: $8.73 \pm 0.59$ , M: $6.63 \pm 0.28)$ was significantly lower in group M. $(\mathrm{C} \mathrm{n}=5, \mathrm{M} \mathrm{n}=$ 3).

Conclusions: The hypoplasia in group $\mathrm{M}$ is not, at least at this stage of malnutrition, caused by apoptosis and necrosis. We suggest the hypothesis that autophagy might be involved in cytopenia observed.

Financial support: FAPESP, CNPQ.

\section{P100}

Effect Antioxidant of Euterpe Oleracea Mart. (Açaí) Extracts on Cell

\author{
Janaína Carvalho Guimarães, Nathália P.C. Soares, \\ Camila C. Brand, Ana Paula M. Rocha, Roberto S. Moura, \\ Anderson Junger Teodoro
}

UNIRIO

Cancer is a complex disease caused by progressive accumulation of genetic mutations and other factors. This disease has a high mortality and prevention strategies have been studied throughout the world. Natural antioxidants, particularly in fruit and vegetables have gained increasing interest among consumers and researchers because epidemiological studies have indicated that frequent consumption of natural antioxidants is associated with a lower risk of cardiovascular disease and cancer. Açaí has antioxidant properties and ability to protect vital cellular components from oxidative stress. The aim of the study was to evaluate the antioxidant activity of seed extract açaí (ESA) on cell viability of human breast, colon and lung adenocarcinoma cell lines (MCF-7, MDA-MB-231, HT- 29 and A549). The antioxidant potential of the ESA assay was used DPPH free radicals. Cells were treated with different concentrations of ESA $(0.3-100$ $\mathrm{mcg} / \mathrm{mL}$ ) for 48 hours. For analysis of cell proliferation, we used the MTT method. ESA were exhibit antioxidant activity around $90 \%$ with an IC50 value of $13.08 \mathrm{mg} / \mathrm{ml}$ in DPPH radical scavenging method. In breast cancer cell lines (MCF-7 and MDA-MB-231), the mean percentage of inhibition of proliferation was $52 \%$ and $74 \%$, respectively. ESA showed a potent reduction on cell viability of lung cancer cell line (A549) in concentrations greater than $50 \mathrm{mcg} / \mathrm{ml}$ with a maximum inhibition of $60.22 \%$. Treatment with ESA in HT-29 cell line showed a lower inhibitory effect and dose-dependent when compared to other cell lines, with reduction up to $44.56 \%$ on cell viability at doses higher than $100 \mathrm{mcg} / \mathrm{ml}$. Our findings showed the capacity of açaí to inhibit cell proliferation in different human cancer cell lines after $48 \mathrm{~h}$. In this context, the chemoprevention of seed extract açaí through the action emerges as an important tool in preventing and controlling cancer. Taken together, these data indicated that the potential anticancer effect of açaí was cellular type and dose-dependent.

\section{P101 \\ Insulin Signaling in LDL Receptor Knockout Mice (LDLR-/-) Fed a Chow or a High-Fat-Diet}

\author{
Jane Cristina Souza, Emerielle Cristine Vanzela, \\ Everardo Magalhães Carneiro, Helena Coutinho F. Oliveira, \\ Antonio Carlos Boschero
}

UNICAMP

Background: Frequently, diabetic patients exhibit dyslipidemia characterized by low levels of high-density lipoprotein, hypertriglyceridemia and increased small dense LDL particles. These modifications in lipoproteins profile may contribute to development of insulin resistance, $\beta$-cell failure, and type II diabetes (T2DM). The use of animal models that exhibit alterations in lipoprotein profile allow us 
to examine how factors that regulate lipid metabolism influences the susceptibility to development of insulin resistance and T2DM.

Objectives: In this study we investigated the insulin signaling in liver, muscle and adipose tissue of $\mathrm{LDLR}^{-/-}$mice, fed a chow or a high-fat diet (HFD).

Methods: Male LDLR ${ }^{-/}$and wild-type (WT) mice, 4 months of age, were fed with HFD (western-type, $40 \%$ Kcal by fat) or a chowdiet for 30 days. Total cholesterol (CHOL), triglycerides (TG), nonesterified fatty acids (NEFA) and plasma insulin were measured by standard commercial kits. Glucose homeostasis was analyzed using oral glucose tolerance test (oGTT) $(1.5 \mathrm{~g} / \mathrm{Kg}$ body weight). Islets were isolated by collagenase and insulin secretion was measured by radioimmunoassay (RIA). Insulin signaling was analyzed by immunopreciptation and immunoblotting of insulin receptor beta (IR $\beta)$ and phosphorylation of protein kinase B (AKT).

Results: $\mathrm{LDLR}^{-/}$mice showed high plasma levels of CHOL, TG, glucose and increased mass of epididymal fat depot compared with WT mice fed a chow diet. The former mice secreted less insulin, were glucose intolerants but more sensitive to insulin than WT, especially in muscle and liver. In LDLR ${ }^{-/}$mice HFD promoted a higher body weight gain. These mice showed a worsened glucose tolerance and impaired insulin signaling as judged by a lower phosphorylation of IR $\beta$ and AKT. In WT mice HFD increased plasma insulin levels but not changed the insulin signaling in liver and adipose tissues.

Conclusions: The results indicate that $\mathrm{LDLR}^{-/-}$mice fed a chow diet are more sensitive to insulin probably due to adaptive mechanisms that compensate lower insulin secretion. However, these changes are not sufficient to maintain glucose homeostasis since these mice were glucose intolerants. When fed a high-fat diet, they became also insulin resistant probably due a large increase in visceral adipose tissue mass.

Financial support: FAPESP (2011/23370-2).

\section{P102 \\ Contribution of Peroxisomes for the Prevention of Diet Induced Obesity and Glucose Intolerance by Fish Oil - Metabolomics Based Evidence}

\author{
Jarlei Fiamoncini, Thaís Martins de Lima, \\ William Tadeu Festuccia, Sandro Massao Hirabara, \\ Renata Gorjão, Talita Romanatto, Alexander Haag, \\ Hannelore Daniel, Rui Curi \\ University of São Paulo
}

Background and Objectives: Fish oil (FO) supplementation has been shown to prevent the development of glucose intolerance and dyslipidemia induced by high fat feeding in mice. These effects are followed by peroxisome proliferation and increases in their activity in the liver. To better understand the FO effects on lipid and carbohydrate metabolism, C57/BL6 mice were fed hyperlipidic diets containing either lard or fish oil (60\% of energy from fat) for 4 weeks.

Methods: After the treatment period, the plasma concentration of glucose, insulin, triacylglycerols, cholesterol and non-esterified fatty acids (NEFA) were quantified. In the liver, the content of triac- ylglycerols, NEFA, acyl carnitines, alkyl-acyl phosphatidylcholines (AAPC) and the expression of genes related to peroxissomal function were measured.

Results: Mice fed the lard-containing diet displayed hyperglycemia and hyperinsulinemia when compared to FO-fed animals. Mice on FO diet had reduced concentrations of plasma triacylglycerols, NEFA and cholesterol as well as lowest concentration of triacylgycerols and NEFA in the liver. Evidence for increased peroxisomal function in the liver of FO fed mice was obtained by a 3-fold increase in mRNA levels for PEXIII (involved in peroxisome biogenesis) and acyl coA oxidase and 2-fold increase for PMP70 (a marker of peroxissomal membrane). Metabolomics applications via LC-MS/MS profiling revealed highest concentration of medium and short-chain acyl carnitines in the liver of FO fed mice, taken as an indicator of increased beta-oxidation of fatty acids by increased activity of peroxisomes. Regardless of the differences in the content of short-chain acyl carnitines, the content of long-chain acyl carnitines remained the same between the groups. In the liver of FO fed mice, the hepatic concentration of total AAPC was increased 2-fold and there was a 3 -fold increase in the AAPC containing only saturated fatty acids. We obtained a very distinct pattern of the AAPC which asks for more studies to define the origin of these changes in particular of the ether lipid fraction of the phospholipids in liver of FO fed mice.

Conclusions: Despite demonstrating a hepatic adaptation with a suggested increase in peroxisome density and activity to a fish oil rich diet in our rodent model, metabolite profiling revealed major changes in various phosphatidylcholine species as well as in acylcarnitines that also affect a variety of other metabolic pathways.

Financial support: FAPESP (PD 2010/20322-4).

\section{P103 \\ Wnt/Beta-Catenin Signaling Is Downregulated But Restored by Nutritional Intervention in Aged Heart in Mice}

\author{
Johnny Li, Steven S. Hannah \\ Nestle Purina Research
}

Background: Aging in general is characterized by decrease in stem cell function and its ability for tissue regeneration. Consequently, the aging heart shows limited ability to adapt to disease or other environmental changes. The Wnt/ $\beta$-catenin signalling pathway has emerged as a key player in cellular aging in recent years. Wnt activity increases in aged tissues including skin, serum, muscle and artery. In heart, it is hypothesized that Wnt signalling increases with aging and this signalling drives cardiac progenitor cells into fibrogenic lineage. However, the molecular mechanism underlying heart aging is poorly understood. Conflicting observations have been reported by various research groups.

Objectives: In this study, we seek to understand the molecular mechanism underlying heart aging in rodent. Methods of nutrition intervention are also explored.

Methods: We take a bioinformatics data mining approach to study rodent gene expression changes in heart aging. Two independent microarray gene expression data sets were identified for this study. A database search is performed to identify nutritents that inter- 
act with beta-catenin, a key regulator of the Wnt signaling. A literature mining is conducted in an attempt to understand the mechanism of this gene and nutrition interactions.

Results/Conclusions: Contrary to the current hypothesis, our study shows that the $\mathrm{Wnt} / \beta$-catenin signalling is down-regulated in aged heart in mice. Nutrition treatment, with calorie restriction (CR) and Resveratrol supplementation, known to retard aging, opposes heart aging by restoring $\mathrm{Wnt} / \beta$-catenin signalling level in the old heart. In addition, the expression of $\beta$-catenin gene, a key regulator of the $\mathrm{Wnt} / \beta$-catenin signaling pathway, decreases up to three fold in aged heart, but is restored to levels found in young heart with methods of nutrition intervention. Combined with database search, our study suggests some of bioflavonoids may have potential therapeutic benefits to heart aging.

\section{P104}

\section{Gene Expression of IL1R1, IL-18 and ICAM-1 in PBMC Has a Positive Predictive Effect on Circulating Levels of TNF-A in Clinically Healthy Adults}

\author{
José Luiz Marques Rocha, Kiriaque Barra Ferr Barbosa, \\ Ana Carolina Pinheiro Volp, Denise Machado Mourão, \\ Caroline Müller, Josefina Bressan
}

Universidade Federal de Viçosa

Background: Peripheral biomarkers of metabolic diseases have been studied in order to identify tools which indicate a possible metabolic prognosis. However, there are gaps about how the inflammatory process could lead to the development of chronic diseases in healthy individuals.

Objectives: To evaluate the gene expression of receptor type-1 of interleukin-1 (IL1R1), interleukin eighteen (IL-18), intercellular adhesion molecule-1 (ICAM1) and tumor necrosis factor alpha (TNF$\alpha$ ) in peripheral blood mononuclear cells (PBMC) of clinically healthy adults.

Methods: This study evaluated 92 women and 68 men with a mean age of $23.2 \pm 3.5$ years and mean BMI of $22.0 \pm 2.9 \mathrm{~kg} / \mathrm{m} 2$. Blood collection was performed by venipuncture after fasting for 12 hours. Isolation of PBMC was accomplished by the method of gradient centrifugation. The extraction of total RNA was made using Trizol $^{\circledR}$ and its concentration and its purity were determined by spectrometer. The integrity of the extracted RNA was verified by agarose gel electrophoresis. The gene expression was performed using the method threshold cycle (TC) comparing, using the real-time PCR (Applied Biosystems), with fluorescence detection SYBR Green. The mean values of triplicate TC's of each sample was used to calculate the expression of the gene of interest normalized by the average of two genes controls ( $\beta$-actin and GPDH). The level of TNF- $\alpha$ was performed by ELISA in EDTA plasma. According to the distribution of variables the Student's t test or the Mann-Whitney-U test was adopted for comparison between the groups categorized by the median of the concentration of TNF- $\alpha$. It was used simple linear regression model to track associations and predictive factors for this biomarker. The software SAS version 9.0 was used to statistical analysis, and it was considered the $5 \%$ of probability.
Results: It was found that expression of IL1R1, IL-18, ICAM1 were significantly correlated with the levels of circulating TNF- $\alpha(\mathrm{r}=$ $0.16, \mathrm{p}<0.001, \mathrm{r}=0.16, \mathrm{p}<0.001, \mathrm{r}=020, \mathrm{p}<0.001$, respectively). After adjustment for possible confounding factors, it was observed that the expression of IL1R1, IL-18 and ICAM1 had a positive predictive effect on circulating levels of TNF- $\alpha(p<0.05)$.

Conclusions: Even in clinically healthy adults, there is a positive correlation between the various markers of inflammation, as evidenced by the prediction of levels of TNF- $\alpha$ from the values of gene expression of mediators involved in this process.

\section{P105 \\ Dietary Açai Up-Regulates Antioxidant Enzymes Expression in Rat Liver}

\author{
Joyce Ferreira Guerra, Maisa Silva, \\ Marcelo Eustáquio Silva, Maria Lúcia Pedrosa
}

Universidade Federal de Ouro Preto

Background: Euterpe oleraceae (açai) is a typical fruit from the Amazon region, largely consumed in Brasil and exported to many countries. Açai gained popularity in United States and others countries after being promoted as a "Superfood" and many health claims have been reported for açai. Such claims are based on phytochemical composition of the berries. Açai is rich in polyphenols most notably anthocyanins, proanthocyanidins and other flavonoids. Dietary polyphenols have been shown antioxidant properties and might offer an indirect protection against oxidative stress by modulate a number of cellular processes including stimulate the transcription of antioxidant defense system. Therefore, açai represents a promising dietary source of phytochemicals and may improve the endogenous antioxidant balance.

Objectives: The present study was undertaken to evaluate the possible protective effects of açai on the liver antioxidant enzymes expression in control and streptozotocin- induced diabetic rats, representing normal physiological conditions and conditions of potentially high oxidative stress.

Methods: Female Fischer rats were divided into four groups, control (C), açai (A), diabetic (D), diabetic + açai (DA). Diabetes was induced by a single intraperitoneal injection of streptozotocin $(35 \mathrm{mg} /$ $\mathrm{kg}$ body weight). Animals in groups $\mathrm{C}$ and $\mathrm{D}$ were fed a standard diet (AIN-93), those in groups A and DA were given the standard diet with $2 \%(\mathrm{w} / \mathrm{w})$ açai pulp added for 30 days. The mRNA expression of liver antioxidant enzymes catalase (CAT), glutathione peroxidase (GPx), gamma-glutamylcysteine synthetase $(\gamma$-GCS), Zn-superoxide dismutase (Zn-SOD) and Mn-SOD was evaluated by qRT-PCR.

Results: Diet supplementation with $2 \%$ açai was found to increase mRNA levels for gamma-glutamylcysteine synthetase and glutathione peroxidase in liver tissue relative to control. Diabetic rats exhibited lower levels of mRNA coding for Zn-superoxide dismutase, glutathione peroxidase and gamma-glutamylcysteine synthetase. The mRNA levels for $\gamma$-GCS in diabetic rats that received acai supplementation did not differ from those in controls.

Conclusions: These findings suggest that acai has a potential protective effect against oxidative stress through the upregulation of liver antioxidant defense system in vivo.

Financial support: $\mathrm{CNPQ}$. 


\section{P106}

\section{Regulation of Gene Expression During Weight Loss}

\author{
Júlia Cristina Cardoso Carraro, Josefina Bressan \\ Universidade Federal de Viçosa
}

About sixty percent of the adult western population search for weight loss, however, the prevalence of obesity still rising. This scenario illustrates the difficulty of overweight individuals maintain the weight lost for long-term. This occurs because the body works to reverse the change of weight, and can be regulated by several factors. It is assumed that in times of food scarcity, individuals have developed, adaptively more gene promoters of food intake and energy storage and that minimize energy expenditure, gene regulators of adipocyte differentiation and, glucose and lipids metabolism. However, this evolution that allowed survival in ancient times, represents an obstacle to weight loss in Western society, seen a sedentary lifestyle and easy access to food. The decrease in body weight promotes an equivalent reduction in energy expenditure, regulated by several factor, such as central nervous system, leptin, gastrointestinal hormones, enzymes involved in lipid metabolism, neuropeptides and transcription factors, such as PPAR gamma 2, C/EBPa and ADD1/ SREPB1. Some studies describe the effect of the weight loss on gene expression of several of these factors in order to reverse the catabolic state: a) the presence of glucose in cell culture 3T3-L1 decreases gaddl 53 expression, that regulates negatively the $\mathrm{C} / \mathrm{EBPa}$, optimizing the action on adipogenesis, b) weight loss after bariatric surgery promotes decreased expression of $11 \beta-H S D 1$, an enzyme related to metabolism of glucocorticoids, whose disorders are associated with obesity, c) in subjects who followed a restricted diet for weight reduction, were found 100 genes regulated by energy restriction, including FABP4, NR3C1, SIRT3 and others, d) obese subjects following weight reduction program had levels of lipoprotein lipase and mRNA in adipose tissue increased, e) obese men and women who underwent bariatric surgery had reduced weight, while women showed lower expression of hormone-sensitive lipase and increased lipoprotein lipase, suggesting a differential response between gender, f ) mice with diet-induced obesity were subjected to calorie restriction for 6 weeks had higher expression of orexigenic neuropeptides NPY and AgRP after weight loss, wich was negatively correlated with leptin levels. All these results illustrate the difficulty of maintaining weight loss in response to therapies used in treatment of obesity and justify the need to study new interventions.

Financial support: FAPEMIG and CNPQ.

\section{P107}

MTOR Modulates the Lipogenesis and the Expression of Nuclear Receptor Coregulators in 3T3-L1

\author{
Juliana Magdalon, Andressa Bolsoni Lopes, \\ Vivian Almeida Paschoal, Thiago Belchior de Oliveira, \\ Rui Curi, William Festuccia
}

Institute of Biomedical Sciences - USP

The comprehension of the mechanisms underlying adipocyte maintenance is essential for the development of therapeutic agents aiming to control obesity and related diseases. This process is modulated by several transcription factors and epigenomic events, which finely regulate gene transcription. Although chronic inhibition of mTORC1 leads to a reduced TAG accumulation in adipocytes, it is still unknown whether acute inhibition of mTORC1 and mTORC2 would trigger the same effects and which mechanisms are involved. Therefore, the aim of this study was to investigate the role of mTORC1 and mTORC2 in the regulation of mature adipocyte lipogenesis and nuclear receptor coregulator expression. For this, 8 days differentiated 3T3-L1 treated for $24 \mathrm{~h}$ with DMSO (control), $100 \mathrm{nM}$ rapamycin (inhibition of $\mathrm{mTORC} 1$ ) or $250 \mathrm{nM}$ torin (inhibition of mTORC1 and 2) were evaluated by either gene expression, or the incorporation of $\mathrm{D}-\left[\mathrm{U}-{ }^{14} \mathrm{C}\right]$-glucose in triacylglycerol (TAG), fatty acids (FA) and glycerol or the incorporation of $\mathrm{D}-\left[\mathrm{U}-{ }^{14} \mathrm{C}\right]$-acetate in fatty acids. mTORC1 and 2 inhibition with torin reduced insulinstimulated glucose incorporation in TAG, FA and glycerol and basal and insulin-stimulated acetate incorporation in FA. The acute inhibition of mTORC1 with rapamycin did not alter lipogenesis in the present conditions. Interestingly, changes in lipogenesis induced by torin were associated with an increase in the mRNA levels of HDAC1, HDAC4, SRC3 and GCN5. The involvement of such gene expression phenotype to the changes in lipogenesis induced by torin is under investigation. In conclusion, mTOR is an important regulator of lipogenesis in mature 3T3-L1 adipocytes.

\section{P108 \\ Effects of Methylseleninic Acid and Selenite on Growth, Histone Post-Translational Modifications and DNA Methylation Status, and Gene Expression of MCF-7 Human Breast Cancer}

\author{
Juliana Xavier de Miranda, Fábia Oliveira Andrade, \\ Aline de Conti, Maria Lúcia Zaidan Dagli, \\ Tiago Franco de Oliveira, Ana Paula de Melo Loureiro, \\ Fernando Salvador Moreno, Thomas Prates Ong \\ University of São Paulo (USP), Brazil
}

Background: Selenium (Se) is a promising dietary anticancer agent. Modulation of epigenetic processes may represent relevant molecular effects by this micronutrient.

Objectives: To investigate effects of two forms of Se [methylseleninic acid (MSA) and selenite (ST)] in MCF-7 human breast adenocarcinoma cells. 
Methodology: Evaluated parameters included cell proliferation (violet crystal assay) and viability (trypan blue exclusion assay), plasma membrane integrity (Flow cytometry), DNA fragmentation (Flow cytometry), apoptosis (Flow cytometry - AnnexinV/Propidium iodide staining), cell cycle progression (Flow cytometry), acetylated (H3K9ac) and trimethylated (H3K9me3) lysine 9 levels on histone $\mathrm{H} 3$, acetylated (H4K16ac) lysine 16 level on histone H4 (Western blot), global DNA methylation (HPLC-DAD), tumor suppressor gene expression (RASSF1a, qPCR) and promoter methylation (RASSF1a, $R A R \beta$, MS-PCR), DNA methyltransferase 1 (DNMT1) expression (Western blot).

Results: Compared to untreated cells (controls), both MSA and ST inhibited $(\mathrm{p}<0.05)$ MCF-7 cell proliferation and viability in a dose- and time-dependent manner. Treatments with MSA favored MCF-7 cell death by apoptosis, that was associated with increased $(p<0.05)$ DNA fragmentation level, and reduced plasma membrane rupture associated with high $(\mathrm{p}<0.05)$ phosphatidylserine exposure. On the other hand, ST increased $(\mathrm{p}<0.05)$ DNA fragmentation, enhanced $(\mathrm{p}<0.05)$ propidium iodide positivity and necrosis $(\mathrm{p}<$ $0.05)$. Se compounds induced cell cycle arrest, increasing $(\mathrm{p}<0.05)$ the proportion of cells in $\mathrm{G} 2 / \mathrm{M}$ phase and reducing $(\mathrm{p}<0.05)$ the proportion of those in G0/G1 and S phases. MSA reduced $(p<0.05)$ H3K9ac and H3K9me3 and increased $(p<0.05)$ H4K16ac. Neither MSA nor ST altered $(\mathrm{p}>0.05)$ global DNA methylation, while both compounds reduced $(\mathrm{p}<0.05)$ DNMT1 protein expression. ST, but not MSA, increased $(\mathrm{p}<0.05)$ RASSF $1 a$ gene expression. In control and Se-treated cells promoter regions of RASSF $1 a$ and $R A R \beta$ were predominantly methylated.

Conclusions: MSA and ST inhibited MCF-7 cell growth through different actions. Epigenetic modulation of histone post-translational modification seems to represent a relevant feature of MSA inhibitory effects in breast cancer cells.

Financial support: FAPESP (2010/03994-9).

\section{P109 \\ Endurance Training Blocks UCP1 Upregulation in Brown Adipose Tissue While Increasing UCP3 in the Muscle Tissue of Rats Fed with a High-Sugar Diet}

Karina Barbosa Queiroz, Amanda Rios Ferreira, Gisele Vieira Rodovalho, Daniel Carvalho de Lima, Juliana Bohnen Guimarães, Cândido Celso Coimbra, Elísio Alberto Evangelista, Renata Guerra-Sá Cota

Universidade Federal de Ouro Preto

Background: The mitochondrial uncoupling proteins (UCPs) of interscapular brown adipose tissue (iBAT) and of muscles play important roles in energy balance. For instance, the expression of UCP1 and UCP3 are modulated by free fatty acid gradients induced by high-sugar diets and acute exercise that is dependent on sympathetic stimulation. However, the effects of endurance training in animals fed with high-sugar diets are unknown.

Objectives: This study aims to evaluate the long-term effects of diet and exercise on UCP1 and UCP3 levels and energy balance efficiency.
Methods: Weaned Wistar rats fed with standard (STD) or highsugar (containing 33\% sweetened condensed milk, HSD) diets were simultaneously subjected to running training (1 hour/day, 5 days/ week in a motor-driven treadmill) over an 8-week period. After the training period, the rats were decapitated, and the iBAT and gastrocnemius muscle tissues were removed for evaluation of the $\beta 3$-receptor, $U c p 1$, and Ucp $3 \mathrm{mRNA}$ and protein expression, which were analyzed by qRT-PCR and western blot, respectively.

Results: Groups fed with an HSD displayed a higher adiposity index and iBAT weight $(\mathrm{P}<0.05)$, whereas exhibited an upregulation of Ucpl mRNA and protein levels $(\mathrm{P}<0.05)$. Training increased $\beta 3$-receptor mRNA in iBAT and reduced the Ucp 3 mRNA in muscle tissues. In association with an HSD, training restored the increasing $\beta 3$-receptor mRNA and greatly upregulated the levels of Ucp3 mRNA. Therefore, training blocked the HSD-induced upregulation of UCP1 expression in iBAT, whereas it upregulated the expression of Ucp 3 mRNA in muscle.

Conclusions: These results suggest that training enhances the relationship between $U c p 1 / U c p 3$ mRNA levels, which could result in higher energy efficiency, but not when HSD-induced elevated sympathetic activity is maintained.

Financial support: CAPES, FAPEMIG, UFOP.

\section{P110 \\ B-Ionone (Bi) Modulates MIR-7A, 18A and 183 in Wistar Rats Submitted to the Resistant Hepatocyte (RH) Model of Hepatocarcinogenesis}

Kelly Silva Furtado, Clarissa Scolastici, Aline de Conti,
Adriana Campos, Aline Henriques,
Robson Francisco Carvalho, Thomas Prates Ong,
Fernando Salvador Moreno
University of São Paulo (USP)

Background: Micro-RNAs are small nonconding RNAs that could inhibit target gene expression by binding to the 3'untranslated region of target mRNA resulting in either mRNA degradation or inhibition of translation. miRNAs play important roles in many normal biological processes, however, studies have also shown that aberrant miRNA expression is correlated with the development and progression of cancers. Dietary isoprenic derivatives such as $\beta$-ionone, a cyclic isoprenoid present in grapes, represent a promising class of cancer chemopreventive agent

Objectives: Previous studies have demonstrated the chemopreventive activity of $\beta \mathrm{I}$ when administered to rats submitted to the $\mathrm{RH}$ model. Therefore, the aim of this study was to analyze the possible modulation of expression of miRNAs in hepatocarcinogenesis by the natural chemopreventive $\beta I$.

Methods: 10 male Wistar rats were submitted to the RH model and treated $(\mathrm{n}=5,16 \mathrm{mg} / \mathrm{Kg}$ b.w., $\beta \mathrm{I}$ group) with $\beta \mathrm{I}$ or corn oil $(\mathrm{n}=$ 5 , controls, $\mathrm{CO}$ group) during the promotion phase for 4 consecutive weeks. Frozen $\left(-80^{\circ} \mathrm{C}\right)$ liver samples were analyzed by the real time PCR (RT2-qPCR) technique. Plates ready for RT2-qPCR (Qiagen ${ }^{\circledR}$ ) were used, containing components and controls of the reaction for detection of 88 miRNAs involved in carcinogenesis. Targets of miR- 
NAs that changed were predicted using the programs TargetScanS, miRanda and Pictar-4.

Results: Compared to $\mathrm{CO}$ group, $\beta \mathrm{I}$ group showed downregulation of miR-7a and upregulation of miR-18a and 183 expression. GATA6 (promoter of cell differentiation) and GLI3 (related to inhibition of activation of $\beta$-catenin) consist in predicted targets of miR-7a. HIF1A (hypoxia-inducible factor-1alpha, related to the increase of cellular proliferation and apoptosis inhibition) and HMGCS1 (related to the synthesis of cholesterol) are targets of miR-18a, and LRP6 (related to activation of $\beta$-catenin in hepatocarcinogenesis) of miR183.

Conclusions: $\beta$ I modulates the expression of miRNAs that regulate genes related to cell differentiation and cancer progression. These actions could contribute to the hepatocarcinogenesis chemopreventive effects of the isoprenoid during promotion.

Financial support: FAPESP (2011/03132-0).

\section{P111 \\ Molecular Aspects of Zinc in Obesity}

Kyria Jayanne Clímaco Cruz, Luana Mota Martins, Ana Raque/ Soares Oliveira, Dilina Nascimento Marreiro

Federal University of Piauí

Obesity is characterized by the presence of a low grade chronic inflammatory process. In recent decades, research has been carried out in the view of understanding hormonal, biochemical and nutritional imbalance present in obese individuals. After sequencing the human genome, genomic studies have focused on elucidating the function of all genes and characterizing their interactions with nutritional factors. Nutrigenomics is one of the most advanced methods that assists in better understanding the cellular homeostasis of zinc. The increasing progress of studies that evaluate the molecular aspects of zinc cell biology by means of proteins that transport zinc (ZnTs and Zips) is a new tool for investigating the nutritional status related to this mineral. These specialized proteins in the uptake, efflux and compartmentalization of zinc appear to be influenced by pro-inflammatory cytokines. The complexity and importance of zinc homeostasis is reflected by the large number of proteins that are potentially dedicated to the transporting of zinc of which at least ten are members of the ZnT family (Zinc Transporter) and 15 are members of the Zip family (ZRT-and IRT-like proteins) and the distinct isoforms of metallothionein. In this sense, zinc acts by modifying the gene expression through its binding to the Metal-responsive transcription factor 1 (MTF-1). With regards to changes in zinc metabolism in obesity, its redistribution from the blood to the liver has been seen. The ongoing inflammatory process favors the increase of the expression of ZIP 14, a protein that promotes the uptake of this mineral from extra intracellular compartment, contributing to hypozincemia in obese individuals. The use of tools such as nutrigenomics may contribute to a better understanding of the influence of obesity on zinc metabolism.

\section{P112 \\ Molecular Mechanisms of Selenium in Breast Cancer}

Kyria Jayanne Clímaco Cruz, Sueli Maria Teixeira Lima, Livia Patrícia Rodrigues Batista, Leiliane Rodrigues Batista, Dilina Nascimento Marreiro

Federal University of Piauí

The pathogenesis of breast cancer involves biochemical and metabolic disorders which contribute to the manifestation of oxidative stress. In recent decades, studies have been carried out with a view to clarify the involvement of micronutrients in the prevention of breast cancer. Selenium, in particular, has been an element of great interest for its role as an antioxidant and anticarcinogenic nutrient. The actions of this mineral are exerted through selenoproteins among which thioredoxin reductase (TRxR), glutathione peroxidase (GPx) and selenoprotein $\mathrm{P}(\mathrm{SePP})$ stand out. The enzyme TRxR, when associated with thioredoxin, forms a redox system with multiple functions, including the formation of deoxyribonucleotides for DNA synthesis and regeneration of ubiquinol-10 antioxidant, which is important in preventing lipid peroxidation. This system has three forms: cytosolic TRxR1, mitochondrial TRxR2 and TRxR3 with activity of glutathione and thioredoxin reductase. This system plays a central role in regulating gene expression through control of transcription factors such as nuclear factor $\kappa \mathrm{B}$, protein activator AP-1 and kinases in apoptosis regulation which acts indirectly by modulating cellular activities such as proliferation, programmed death of cells and activation of the immune response. The GPx group has four currently known members: cytosolic GPx1, which reduces hydrogen peroxides and hydroperoxides, gastrointestinal GPx2, renal GPx3 and GPx4, which reduce lipid hydroperoxides. These enzymes exert their action from the reduced glutathione. The SePP has three basic functions: carry selenium from the liver to the kidneys, bind to heavy metals, especially mercury, and have antioxidant activity in vitro and in vivo with the reduction of phospholipid hydroperoxides and inhibition of the oxidation of low density lipoproteins. In this regard, variation in the genes that encode the selenoproteins could influence interindividual differences in the metabolism of selenium and in mechanisms involved in the synthesis of these selenoproteins. Recent studies have shown that reduced expression of selenoproteins increases the susceptibility to the development of mammary tumors, demonstrating the chemopreventive action of selenium. Thus, nutrigenomics is an important tool for understanding the molecular and metabolic disorders present in breast cancer. 


\section{P113 \\ Role of Vitamin D in Molecular Mechanisms in the Secretion and Action of Insulin in the Obese}

\author{
Kyria Jayanne Clímaco Cruz, Fabiane Araújo Sampaio, \\ Dilina Nascimento Marreiro
}

Federal University of Piauí

Obesity is a chronic disease characterized by an excessive accumulation of body fat and the presence of insulin resistance and lowgrade chronic inflammation. Some studies have shown an anti-inflammatory role for vitamin $\mathrm{D}$ in adipogenesis and insulin resistance in obese individuals. The mechanisms that may clarify the role of this vitamin in the manifestation of these disorders include indirect post-transcriptional effects by stimulating calcium influx in pancreatic $\beta$-cells and in the direct transcriptional regulation mediated by activation of the nuclear receptor of vitamin D, VDR. The action of the VDR is based on the recognition of a specific sequence in the DNA strand, the element responsive to vitamin $\mathrm{D}$, where there are several genes, such as insulin, which allow the coupling of VDR with retinoic acid receptor (RXR). The VDR receptor is expressed by many cell types involved in the inflammatory process, such as monocytes and macrophages, which suggests the effect of vitamin D deficiency in increasing the inflammatory response associated with insulin resistance in the obese. Vitamin D regulates IK $\beta$ - $\alpha$, a $\beta$-NFK inhibtor, which is a transcription factor involved in gene regulation that encode proinflammatory cytokines related to insulin resistance. In this respect, it has been observed that the increase in the secretion of proinflammatory cytokines induces stress in pancreatic $\beta$ cells contributing to apoptosis. Supplementation with vitamin D reduces the concentrations of IL-1 $\beta$ and IL-15 and blocks the apoptosis of pancreatic $\beta$ cells, which contribute to the improvement of insulin secretion. This vitamin reduces the migration and the recruitment of $\mathrm{T}$ cells and macrophages to the islets, modulating the effects and production of cytokines. However, some studies have found no reduction in cytokine concentrations in obese individuals supplemented with vitamin D. Therefore, it is necessary to carry out further studies on the subject to clarify the benefits of the use of vitamin D supplements in order to favor insulin secretion and action and reduce the inflammation present in the obese.

\section{P114 \\ The Influence of Gustin Gene Polymorphism on the Nutritional Status}

Sueli Maria Teixeira Lima, Kyria Jayanne Clímaco Cruz, Livi a Patricia Rodrigues Batista, Leiliane Rodrigues Batista, Nadir Nogueira Nascimento, Dilina Nascimento Marreiro

Federal University of Piauí

In addition to the metabolic conditions and lifestyle, the existence of differences in the perception of taste that may determine food choices between obese and normal weight individuals has been suggested. The salivary proteins are involved in taste and could be correlated with the different perceptions. Among them, Gustin or carbonic anhydrase VI (CA6), a zinc metalloproteinase secreted by the serous acinar cells of the parotid, submandibular and von Ebner glands, may play a crucial role in sensitivity to taste. Some authors have suggested that gustin is a trophic factor that promotes growth and development of taste buds. A reduction in the secretion of salivary gustin was associated with decreased or distorted function of taste and smell. The enzymatic function of gustin depends on the presence of zinc in its active site. Accordingly, treatment with zinc can improve the function of flavor in the elderly, increase the concentration of gustin in subjects with hypogeusia and normalize the function of taste and morphology of the taste buds in patients with deficient CA6. Recent discoveries have shown that the polymorphism rs2274333 (A / G) of gustin is involved in the modulation of its activity due to the location of this polymorphism in exon 3 of the gustin gene, which encodes amino acid residues of the active zinc site. Therefore, the zinc binding protein is essential for the function of gustin. Studies indicate that the recessive and less functional polymorphism rs2274333 (A / G) is related to decreasing the function of taste, high zinc concentration of saliva and thus poor nutritional status. Some researchers have found a correlation between excess weight and a decrease in the perception of basic tastes in adults. Gustin gene genotyping may represent a specific and reliable marker for individual differences in the perception of flavor and constitutes a research tool in studies which aim to assess eating behavior, impairment of taste function and nutritional status.

\section{P115 \\ Body Mass Index and Global Methylation in Leukocyte DNA in a Prospective Cohort}

\author{
L. Joseph Su, Sonja I. Berndt, Lee E. Moore, \\ Hormuzd A. Katki, Srinivasan Yegnasubramania, \\ Mark P. Purdue, Wen-Yi Huang
}

National Cancer Institute

Background: It has been hypothesized that there is a link between epigenetics and obesity. Studies have demonstrated that animals fed with methyl-deficient diet resulted in alterations of promoter DNA methylation and leads to offspring obesity. However, evidence from epidemiologic studies is scarce, prospective investigations of this relationship is particularly lacking.

Objectives: This study examines whether obesity, measured by body mass index (BMI), is associated with epigenetic changes, measured by the extent of methylation in cytosine (5-mC) and LINE-1 among healthy subjects.

Methods: Leukocyte DNA was measured for methylation of 5-mC and LINE-1, among 519 control subjects, who were 55-74 year old at study entry and free of any cancer at the time of blood collection, in a nested case-control study of colon cancer within the Prostate, Lung, Colorectal, and Ovarian (PLCO) Cancer Screening Trial. Selfreported height and weight at study baseline, age 50, and age 20 were used to calculate BMI at three time points in life.

Results: Spearman correlation adjusted for age, gender, race, study year, and smoking status showed that LINE-1 methylation, when analyzed as a continuous variable, was significantly and inversely correlated with BMI at baseline $(r=-0.11, p=0.01)$ and age 
$50(\mathrm{r}=-0.11, \mathrm{p}=0.02)$ and marginally significantly with BMI at age 20. Similarly, when examining the methylation status categorized by tertile, LINE-1 methylation was inversely correlated with BMI in more recent measurements but not BMI at age 20. Multivariate logistic regression models demonstrated that low LINE-1 methylation level was positively associated with obesity $(\mathrm{BMI}>30)$ at baseline (3rd vs. 1 st tertile: odds ratio $=2.23,95 \%$ confidence interval: 1.24 , 3.98 , test for trend $\mathrm{p}=0.007$ ). No clear association was found between 5-mC and obesity.

Conclusions: This unique study examined the relationship between BMI at three time points in life and two different global methylation measurements in Leukocyte DNA in a cancer-free population. Recent obesity appeared to correlate with LINE-1 hypomethylation status.

\section{P116}

Contribution of the TNF-Alpha Receptor 1 in Insulin Sensitivity and Glucose Tolerance in Mice Fed a High Refined CarbohydrateContaining Diet

\author{
Laís Bhering Martins, Zélia Menezes Garcia, \\ Marina Chaves Oliveira, Raquel Duque Arifa, \\ Renata Lacerda Lima, Mauro Martins Teixeira, \\ Leda Quercia Vieira, Danielle Glória Souza, \\ Adaliene Versiani Ferreira \\ UFMG
}

Background: The Tumor Necrosis Factor-alpha (TNF- $\alpha$ ) is a proinflammatory cytokine produced mainly by macrophages. This cytokine modulates the metabolism of nutrients by stimulates lipolysis, regulates adipocyte function and insulin signaling (Volp, A. et al., 2008, Olson, N. et al., 2012).

Objectives: The aim of this study was to verify whether the lack of TNF-alpha receptor 1 alters the body weight gain and adiposity, as well as peripheral insulin sensitivity and glucose metabolism in mice fed a high refined carbohydrate-containing diet (HC).

Methods: Mice with genetic deletion of TNF-alpha receptor 1 (TNF- $\alpha$ R1-/-) and wild-type mice (WT) were divided into groups according to experimental diets: chow $\operatorname{diet}(\mathrm{C})$ or high refined carbohydrate-containing diet (HC). After 8 week of dietary treatment, fasting serum glucose was assessed from tail blood samples and the animals were submitted to Insulin Sensitivity Test (IST) (75Ul/ kg body weight) and Oral Glucose Tolerance Test (OGTT) (2 g of D-glucose / kg body weight). Three days after testing, mice were euthanized, the epididymal, retroperitoneal and mesenteric adipose tissue were weighed and the adiposity index determined.

Results: Mice fed HC diet presented increased adiposity. No significant difference in fasting serum glucose was observed between groups. WT-HC mice presented lower glucose tolerance and insulin sensitivity when compared to the WT-C. However, TNF- $\alpha$ R1-/-HC mice presented similar insulin sensitivity and tolerance to glucose when compared to mice fed chow diet.

Conclusions: The results showed that despite the absence of TNF-alpha receptor 1 did not change the obesity state, it can be associated to the development of insulin resistance induced by high refined carbohydrate-containing diet.

\section{P117}

\section{New Evidence for Anthocyanins, Atherosclerosis and Nutrigenetics}

\author{
Larissa Lovatto Amorin, Natália Cruz Campos, \\ Marina Andrade Batista, Gisele Araújo Magalhães, \\ Marialice Pinto Silvestre
}

\section{Universidade Federal de Minas Gerais}

This review is about the benefits of anthocyanins in modulating atherosclerosis, which is the leading cause of morbimortality worldwide. This pathologic condition is related to vessel-wall adhesion, platelet activation, aggregation and the development of inopportune thrombi ${ }^{1}$. Recent studies have shown that foods rich in anthocyanins like black rice, red propolis and bilberry are able to inhibit platelet function ${ }^{1,2,3}$. Dyslipidemic rats receiving dietary supplementation with anthocyanin extract from black rice significantly presented reduced body weight gain and restored serum triglyceride levels and platelet function by decreasing calmodulin and sP- selectin levels, thromboxane A2 production and thromboxane A2: prostacyclin ratio $^{1}$. In another study, the bilberry anthocyanin-rich extract added to the diet of apo $\mathrm{E}^{-/-}$knockout mice showed hypocholesterolemic effects $^{2}$. The green, red and brown propolis containing polyphenols were given by gavage ( $250 \mathrm{mg} / \mathrm{kg}$ per day) to LDL receptor gene $\left(\mathrm{LDLr}^{-/}\right)$knockout mice and showed to reduce atherosclerotic lesions through mechanisms including the modulation of inflammatory and angiogenic factors, specially the red one ${ }^{3}$. Therefore, further studies are needed involving anthocyanins to prove its positive actions in preventing or reducing atherosclerotic lesions and to explain the molecular mechanisms involved.

References: 1. Yang Y, Andrews MC, Hu Y, et al. Anthocyanin extract from black rice significantly ameliorates platelet hyperactivity and hypertriglyceridemia in dyslipidemic rats induced by high fat diets. Journal of Agricultural and Food Chemistry 2011;59:67596764.

2. Mauray A, Felgines C, Morand C, et al. Bilberry anthocyaninrich extract alters expression of genes related to atherosclerosis development in aorta of apo E-deficient mice. Nutrition, Metabolism \& Cardiovascular Diseases 2012;22:72-80.

3. Daleprane JB, Freitas VS, Pachecoc A, et al. Anti-atherogenic and anti-angiogenic activities of polyphenols from propolis. Journal of Nutritional Biochemistry 2012;23:557-566.

Financial support: CNPQ and FAPEMIG. 


\section{P118 \\ The Effect of Fish Oil Supplementation on Insulin Sentivity Involves a TLR-4-Dependent Mechanism}

\author{
Laureane Nunes Masi, Amanda Roque Martins, \\ Amanda Rabelo Crisma, Catia Lira do Amaral, \\ Jose Cesar Rosa Neto, William Festuccia, \\ Sandro Massao Hirabara, Rui Curi \\ Universidade de Sao Paulo
}

Toll-like receptors (TLRs) are a family of pattern-recognition receptors that play a critical role in the innate immune system by activating proinflammatory signaling pathways in response to microorganisms or endogenous ligand nonesterified fatty acids. Although some authors demonstrated that TLR4-mutated mice are protected against inflammation and insulin resistance (IR) when challenged with high-fat diets (HFD), others showed that TLR4 deletion promotes obesity, IR and a diabetic phenotype in mice. The incorporation of n-3 polyunsaturated fatty acids into an obesogenic diet attenuates the accumulation of body fat, IR and inflammation in $\mathrm{C} 57 \mathrm{Bl} / 6$ mice but the mechanism involved remains to be found. The aim of this study was to investigate the effect of the pre-treatment with fish oil (FO) on obesity and IR induced by a HFD in TLR-4 knockout $(\mathrm{KO})$ mice. Control $(\mathrm{C} 57 \mathrm{Bl} / 6)$ and TLR4 KO male mice were used. The FO supplementation (EPA/DHA 5:1, 2 g/Kg b.w, 3 times per week) was carried out for 12 weeks. The animals received a balanced diet (BD) until to the fourth week of FO supplementation. After this period, the animals were maintained in a $\mathrm{BD}$ or submitted to a HFD (59\% fat) for 8 weeks. The following parameters were mesured: body weight gain, fat depots weight gain, food intake, glucose tolerance and insulin sensitivity tests. HFD given for 8 weeks increased the body weight, the mesenteric, retroperitoneal and epididymal fat depots, and 12h-fasting blood glucose levels (by 20\%), all compared to BD. Concomitantly, impairments in glucose tolerance and insulin sensitivity were found. The FO supplementation for 12 weeks attenuated the increase in body weight induced by HFD and improved the insulin sensitivity. In TLR4 KO mice, the HFD increased the body weight gain, the retroperitoneal and epididymal fat depots, and 12h-fasting blood glucose levels (by 30\%), and impaired the glucose tolerance test, all compared to BD. The FO supplementation did not protect against the effects induced by the HFD in TLR4 $\mathrm{KO}$ as observed in wild-type $\mathrm{C} 57 \mathrm{Bl} / 6$ mice. In fact, TLR4 KO fed the HFD for 8 weeks even under supplementation with FO showed increased body weight gain, raised mesenteric fat depot, and impairments in glucose tolerance and insulin sensitivity tests, all compared with wild-type mice under HFD. In conclusion, FO protected $\mathrm{C} 57 \mathrm{Bl} / 6$ mice from the increased body weight gain induced by a HFD and improved the insulin sensitivity through a TLR4 mediated mechanism.

Financial support: FAPESP (2010/08872-9).

\section{P119 \\ Anti-Inflammatory Effect of Vitamin D on Prostate Cancer}

Leiliane Rodrigues Batista, Lívia Patrícia Rodrigues Batista,

Kyria Jayanne Clímaco Cruz, Sueli Maria Texeira Lima,

Aline Naiara Sousa Santos, Nara Vanessa Anjos Barros,

Adriana Azevedo Paiva

Universidade Federal do Piauí

Cancer is an important public health issue, prostate cancer is the most prevalent among men, especially in the elderly. Inflammation in the prostate has been proposed as an etiological factor in the development of this cancer, because activation of signaling pathways in tumor tissue. There is evidence of antineoplastic activity of calcitriol (the active form of vitamin D), both through receptor VDR (vitamin $\mathrm{D}$ receptor) as for its anti-inflammatory, with beneficial effects in this type of cancer. Research developed in prostate tumor cells indicated that the calcitriol exerts regulatory effects on major molecular pathways related to inflammation. These include inhibition of synthesis and biological actions of prostaglandin (PG), chemical mediator responsible for the activation ways of proliferation, angiogenesis and other pro-carcinogens. The mechanisms by which calcitriol inhibits the action of pro-inflammatory prostaglandins occurs through the suppression of expression of cyclooxygenase-2 (COX-2), an enzyme that synthesizes PGs, upregulation of expression of the enzyme that inactivates PGs, 15-hydroxyprostaglandin dehydrogenase (15PGDH) and downregulation of PG receptors essential in their signaling mechanisms. Another molecular way in which the calcitriol acts is MKP5. Calcitriol increases the expression of MKP5, the enzyme responsible for inactivation of mitogen-activated protein kinase (MAPK), specifically p38 MAPK, responsible for the production of inflammatory mediators such as cytokines and IL-6, implicated in the pathogenesis of cancer, reducing them. Other mechanisms are, blocking the activation of NF-kB with consequent reduction of interleukin-8, a reduction in the expression of matrix metalloproteinase 9 (MMP-9), suppression of pro-angiogenic factors such as vascular endothelial growth factor (VEGF), leading to inhibition of invasion and metastasis by these routes. Therefore the anti-inflammatory activity of vitamin D appears as an important additional way for the prevention and treatment of prostate cancer, being necessary to the achievement of a greater number of studies to clarify how it should be given their participation in the oncologic therapy type of this cancer.

\section{P120 \\ Current Aspects of Calcium in Obesity \\ Leiliane Rodrigues Batista, Livia Patrícia Rodrigues Batista, Jéssica A. Soares Carvalho, Micaelle Oliveira Vieira, Tatiana Abreu Barros, Kyria Jayanne Clímaco Cruz, Sueli Maria Texeira Lima \\ Universidade Federal do Piauí}

Obesity is a chronic disease of multifactorial etiology, with emphasis on genetic and environmental factors associated with the risk of hypertension, cardiovascular disease, type 2 diabetes, some 
cancers and other comorbidities. Recent studies suggest that the metabolism of calcium and probably other components of dairy products, can help change the energy balance and thus act in the regulation of body weight. One mechanism that may explain the influence of calcium on weight and body fat percentage is your relationship with levels of parathyroid hormone (PTH) and 1,25-dihydroxy vitamin D3 or calcitriol. Thus, low dietary intake of calcium and its lower absorption induces increased levels of PTH and D3. These, in turn, increase the intracellular influx of calcium in adipose tissue, which stimulates the expression of the enzyme fatty acid synthase, promoting lipogenesis, and inhibits the phosphorylation of hormone sensitive lipase, reducing lipolysis and oxidation of fats. The intracellular calcium stimulates the production of proinflammatory cytokines in adipose tissue and skeletal muscle. The calcium supplementation may also promote increased body temperature and the expression of uncoupling protein UCP-2 and decrease in efficiency of energy use. It can also bind to the fatty acids in the intestine, forming insoluble complexes with feces excreted by the consequent reduction of fat absorption.

Therefore, further studies about the nutrigemonics use are needed in order to contribute to the understanding of the calcium action on obesity.

\section{P121 \\ Molecular Mechanisms of Chromium on Insulin Action in Type 2 Diabetes Mellitus}

Leiliane Rodrigues Batista, Livia Patrícia Rodrigues Batista, Kyria Jayanne Clímaco Cruz, Jéssica A. Soares Carvalho,

Tatiana Abreu Barros, Micaelle Oliveira Vieira,

Sueli Maria Texeira Lima

Universidade Federal do Piauí

The Type 2 Diabetes mellitus is a chronic disease characterized by abnormalities in insulin secretion, hepatic glucose overproduction and insulin resistance in peripheral tissues. Some studies have shown chromium as a nutrient that plays role of potentiating effects of insulin in diabetic individuals. A proposed mechanism of action for chromium has as a key player chromium oligopeptide which binds the chromodulin. In response to increased plasma insulin levels after intake of carbohydrates, transferrin receptor translocate to the plasma membrane while the chromium on transferrin, moves to the cells, the complex being internalized by endocytosis and separated by $\mathrm{pH}$ changes in the vesicle. The chromodulin, stored in the cytosol and nucleus in its inactive form (apo-chromodulin), binds to chromium free, forming the holocromodulina, active form. Insulin binds to its receptor, activating it and allowing it to exercise its functions. The holocromodulina binds to $\beta$ subunit of the insulin receptor (IR), assisting in the maintenance of its conformation and amplifying its activity cinásica. Amplification of the phosphorylation of IR by chromium has been demonstrated in vivo and appears to increase in a dependent dose. Research indicates that holocromodulina also inhibits the phosphotyrosine phosphatase $1 \mathrm{~B}$, which is responsible for the dephosphorylation of a substrate and the IR of the insulin receptor (IRS-1). The activation of the kinase and phosphatase inhibition results in increased phosphorylation of IR and hence greater sensitiv- ity to the hormone. When insulin concentrations reduce the chromodulin is eliminated from the cells, which is consistent with the fact that after carbohydrate intake, urinary chromium levels increase. Another possible mechanism of action includes chrome effect post-receptor phosphorylation by protein kinase $\mathrm{B}$ and activation of adenosine monophosphate kinase (AMPK), promoting the translocation of vesicles containing GLUT-4, which results in increased glucose utilization However, the scientific evidence concerning the action of chromium in diabetic patients remains controversial, and therefore there is need for further studies to clarify the actual mechanism by which this mineral promotes improvement to the insulin sensitivity in these patients.

\section{P122 \\ Identification and Expression Analysis of FBPA Gene in Lactobacillus Delbrueckii UFV H2B20}

\author{
Lisiane Lopes Conceição, Eliana Santos Leandro, \\ Fernanda Souza Freitas, Tatiana Fiche Teixieira, \\ Marcelo Nagem Oliveira, \\ Maria Do Carmo Gouveia Peluzio, \\ Alessandra Barbosa Ferreira, Arnaldo Chaer Borges, \\ Célia Alencar Moraes
}

Universidade Federal de Viçosa

Bacteria of the genus Lactobacillus are inhabit the human gastrointestinal tract (GIT) and species such as Lactobacillus delbrueckii UFV H2b20, isolated from feces of infant exclusively fedbreast milk, have physiological, technological, immunological and genetic characteristics desirable to probiose. Probiotics are microorganisms that when administered in adequate amounts confer a health benefit on the host.The objective of this work was to study the expression of the gene $f b p A$, encoding the binding protein fibronectin, involved in adhesion to the human gastrointestinal tract.Total DNA extracted from $L$. delbrueckii UFV H2b20 was submitted to PCR with primers based on the $f b p A$ sequence from $L$. delbrueckii subsp. bulgaricus ATCC 11842. The resulting amplicon was cloned and sequenced. The PCR inverse was used in order to identify and amplify the DNA sequences flanking the internal region of the gene $f b p A$ obtained by PCR. Phylogenetic tree was reconstructed by Bayesian Inference. Cells of L. delbrueckii UFV H2b20 incorporated in fermented milk was exposed to the conditions prevailing in the GIT and RNA was extracted from the cells according to the manufacturer's instruction for using Trizol (Invitrogen ${ }^{\circledR}$ ). cDNA synthesis was performed using the kit ImProm-IITM Reverse Transcription System (Promega ${ }^{\circledR-}$ ) and Real-time PCR using the kit Platinum ${ }^{\circledR} \mathrm{SYBR}^{\circledR}$ Green qPCRSuperMIx-UDG (Invitrogen ${ }^{\circledR}$ ) and primers constructed based on the fbpA sequence from L. delbrueckii UFV H2b20.The 1099 base pairs sequence revealed a fbpA homolog, confirming its presence in $L$. delbrueckii UFV H2b20. This sequence presented $98 \%$ identity with the gene encoding the FbpA in L. delbrueckii subsp. bulgaricus ATCC 11842 available in GenBank with accession number CN: 114846. The presence of $f b p A$ in L. delbrueckii UFV H2b20 can mean advantage its ability to colonize the intestine.The $f b p A$ gene from $L$. delbrueckii UFV H2b20 grouped with the gene from L. delbrueckii 
subsp. bulgaricus ATCC 11842. The expression of the $f b p A$ gene in $L$. delbrueckii UFV H2b20, incorporated in fermented milk, after 240 minutes exposure to the conditions prevailing in the GIT, was reduced but this result does not rule out the involvement of this gene in response to stress imposed by the simulation of the human GIT. The results demonstrate that $L$. delbrueckii UFV H2b20 has the fbpA gene, which may mean a comparative advantage in capacity to colonize the intestine.

\section{P123}

\section{Cardioprotective Effect of Maná-Cubiu Fruit (Solanum Sessiliflorum Dunal) in Wistar Rats}

\author{
Livia Cristina Hernandes, Mara Ribeiro Almeida, \\ Joana D. Arc Castania Darin, Regislaine Valéria Burim, \\ Eliseu Rodrigues, Adriana Zerlotti Mercadante, \\ Maria Lourdes Bianchi, Lusânia Maria Greg Antunes \\ Universidade de São Paulo - FCFRP
}

Background: Diet plays a pivotal role in promoting health. Higher intake of fruits and vegetables has been associated with reduced risk of cardiovascular diseases, diabetes and even cancer. A range of studies have investigated the protective effects of fruits against DNA damage in different organs as liver, heart and kidneys. However, many fruits remain unexploited by scientific community despite their potential action on preventing genomic instability. Maná-cubiu (Solanum sessiliflorum Dunal), a native fruit from Amazon Forest, is used in folk medicine to control blood glucose and cholesterol levels. Nevertheless, lack of scientific information and its antioxidant potential represent some of the reasons to evaluate this promising fruit.

Objectives: The aim of this study was to investigate the effects of maná-cubiu in the heart of Wistar rats by the comet assay, used to detect genomic instability.

Methods: The tests were performed with three doses of manácubiu pulp $(125,250$ or $375 \mathrm{mg} / \mathrm{kg}$ b.w.) by gavage for 14 days, followed by intraperitoneal injection (i.p.) of saline or doxorubicin (DXR, $16 \mathrm{mg} / \mathrm{kg} \mathrm{b.w}$ ), just after the last gavage and 24 hours before euthanasia. Heart was removed and weighed to establish the relationship between heart and body weight, which can reveal toxicological alterations. The comet assay was performed under alkaline conditions and 100 nucleoids were analyzed per animal by Comet Assay IVTM, using the Tail intensity parameter.

Results: The animals treated with maná-cubiu did not differ significantly $(p>0.05)$ in body and organ weight compared with the control group. There was no genotoxic effects in the animals treated with maná-cubiu at all doses, in comparison with negative control $(\mathrm{p}>0.05)$. Moreover, the administration of maná-cubiu resulted in a significant reduction $(p<0.05)$ in DNA damages index induced by DXR, when compared with animals treated with a single dose of DXR.

Conclusions: The administration of maná-cubiu fruit at all three doses tested was effective against DXR-induced DNA-damage in heart cells. The cardioprotective effects of maná-cubiu must be better investigated in others conditions and we are currently performing an evaluation of iNOS and COX-2 gene expression in the heart. Here, we suggest that bioactive compounds such as carotenoids and phenolic compounds could be responsible for these protective effects. Financial Support: FAPESP and CAPES.

Financial support: FAPESP (2011/01982-6).

\section{P124 \\ Genomic Action of Retinoic Acid in Cervical Cancer}

Livia Patrícia Rodrigues Batista, Leiliane Rodrigues Batista, Micaelle Oliveira Vieira, Jéssica A. Soares Carvalho, Tatiana Abreu Barros, Kyria Jayanne Clímaco Cruz, Sueli Maria Texeira Lima, Adriana Azevedo Paiva

Universidade Federal do Piauí

The cervical cancer is the third most common malignancy among women, as primary etiological factor Human Papillomavirus (HPV), considered only an initial step, being insufficient to promote cancer progression. Some studies have confirmed the anticarcinogenic effect of retinoic acid (RA), active metabolite of vitamin A which regulates the rate of growth, differentiation and apoptosis and suppresses the effects of nuclear transcription factors such as activator protein 1 and NF-IL6, expressed capable of proliferation and inflammation. The action to prevent cervical cancer by RA may be a consequence of its ability to modulate gene expression. The effects of retinoids are mediated by two classes of receptors, the retinoic acid receptors (RARs) and retinoid X's (RXRs), each subclass $\beta, \alpha$ and $y$. RAR receptors have a strong affinity for all isomers and trans 9-cis RA whereas RXRs showed strong specificity only for the 9-cis isomers. These nuclear receptors induce the transcription of target gene by binding to response elements in the promoter region. RARs and RXRs are able to interact with other nuclear receptors, and thus expand its action on gene expression. However, the efficiency depends on the RA receptors and their interaction with other factors and cofactors. The RARs are complexed by heterodimerization with RXRs and act as ligandinducible response elements (Rares) and elements of response to retinoid X (RXREs) in the promoter regions of genes responsive to RA. Both the positive and negative regulation of gene retinoids have been identified. The high level of RAR $\alpha$ favors the binding of activated heterodimer RXR / RAR RAR selective retinoids. However, RAR coactivators and co-repressors are also involved in the efficient transcription of genes responsive to RA. Thus, studies of vitamin A showed its protective effect, especially in the early stages of carcinogenesis, against the persistence and subsequent progression of HPV infections. However, there is still controversy about the action of vitamin A, being necessary to conduct new studies on the subject in order to clarify the benefits of vitamin A supplements in order to favor the prevention of cervical cancer uterus. 


\section{P125}

\section{Molecular Aspects of Vitamin D on Cancer}

Livia Patrícia Rodrigues Batista, Leiliane Rodrigues Batista, Kyria Jayanne Clímaco Cruz, Aline Naiara Sousa Santos,

Suely Maria Texeira Lima, Adriana Azevedo Paiva

Universidade Federal do Piauí

Cancer is a chronic disease characterized by abnormal growth of cells due to changes in genetic material, related to endogenous and exogenous factors. Some studies have shown that vitamin D exerts non calcemics activities associated with the gene modulation, with physiological and pathological implications for the development of cancer, especially breast, prostate and colorectal. Among the main evidence for the anticancer action of vitamin $\mathrm{D}$, it is included direct transcriptional effects mediated by direct transcriptional activation of the nuclear vitamin $\mathrm{D}$ receptor (VDR). The active form of vitamin, $1.25(\mathrm{OH}) 2 \mathrm{D}$, upon binding to its receptor induces the heterodimerization of the retinol receptor (RXR), forming the RXR-VDR complex, is then translocated to the nucleus, where it promotes transcription genes that have a response element of vitamin D (VDRE) in its promoter region. The 1,25 (OH) 2D has anti-proliferative effect by means of gene expression CDKN1 which encoding the tumor suppressor CKI p21 and repression factor pro-proliferative tumor, such as c-myc and K16. In addition it provides anti-tumor effect resulting from the adjustment key mediators of apoptosis, as the repression of expression of proteins anti-apoptotic BCL2 and BCLXL and induction of pro-apoptotic protein BAX, BAK and BAD. In normal cells, pro-apoptotic properties of 1,25 (OH) $2 \mathrm{D}$ are essential in order to control the hyperplastic growth. Other antineoplastic mechanism of vitamin D is the deletion of genes encoding proinflammatory mediators such as cytokines, chemokines and prostaglandins, which are capable of increasing the tumorigenesis through the activation of signaling pathways in tumor tissue. Finally, studies have shown an association between low levels of calcitriol (1,25 (OH) 2D) and presence of cancer and improve the prognosis and patient survival when supplemented with vitamin D. Many aspects related to the antineoplastic effect of vitamin D have to be clarified so that its supplementation can be inserted as a clinical practice routine in the treatment of neoplasia.

\section{P126 \\ Role of Vitamin D in Type 1 Diabetes Mellitus}

\author{
Livia Patrícia Rodrigues Batista, \\ Aline Naiara de Sousa Santos, Leiliane Rodrigues Batista, \\ Janaína Guimarães Barbosa, Kytia Jayanne Rodrigues Batis \\ ta, Sueli Maria Texeira Lima \\ Universidade Federal do Piauí
}

Several studies have shown activities or functions of vitamin D is not related to bone metabolism, with effects on various types of illnesses, including autoimmune disorders. Among these, the Type 1 Diabetes Mellitus (Type 1DM) is a complex disease, involving genetic and environmental factors that lead to autoimmune destruction of beta cells. Recently, it has been demonstrated that vitamin D shows, among other functions, immunomodulating activity, which acts by stimulating phagocytosis and killing of bacteria and the reduction capacity of antigen presentation by dendritic cells in vitro and production of cytokines. The enzyme 1-alpha hydroxylase responsible for the activation of the metabolite $25(\mathrm{OH}) \mathrm{D}$ to $1,25(\mathrm{OH}) 2 \mathrm{D} 3$, is present in most immune cell, while the vitamin D receptor (VDR) is expressed in macrophages, antigen-presenting cells and activated $\mathrm{T}$ lymphocytes. Recent studies have considered as markers of genetic susceptibility of Type 1DM four polymorphisms (BsmI, FokI, ApaI, TaqI) in the VDR gene. Results suggest that the genotype "BB" of the BsmI polymorphism, genotype " $\mathrm{tt}$ " Talq polymorphism and higher frequency of allele $\mathrm{F}$ in polymorphism Fokl are associated with type 1DM. Many studies have demonstrated an increased susceptibility to Type 1DM in the presence of the BsmI polymorphism in certain populations. This can be explained by genetic influences and environmental factors that are critical in the activation of genes for certain diseases. In contrast, other studies found no association between polymorphisms Fokl, ApaI and Talq with the presence of Type 1 DM. Although more research is needed to confirm these findings. The vitamin D deficiency appears to be related to the pathogenesis of type 1 diabetes, because of its effect shaping of the immune system and regulating the differentiation and proliferation. The supplementation of this vitamin since childhood, reduces the risk of Type 1 DM. Thus, the effects that vitamin D has on the immune system, some studies highlight the important role of this hormone in the prevention of this disease. Besides the influence of environment, genetic polymorphisms implicated in autoimmune diseases determine variations in their susceptibility and bioavailability of vitamin D among individuals. The clear identification of genotypes at risk for Type 1DM and understanding of the role of vitamin $\mathrm{D}$ on this pathology may be critical in defining therapeutic and preventive strategies against the disease.

\section{P127 \\ Creatine Supplementation and Homocysteine Metabolism in Rats Fed Choline-Deficient Diet}

\author{
Lucas Vieira Francisco, Rafael Deminice, \\ Alceu Afonso Jordão Jr., Lilian Eslaine da Silva \\ University of São Paulo
}

Backgrounds: In recent years, hyperhomocysteinemia (HHcy) has been related to the development of chronic kidney disease, diabetes and nonalcoholic steatohepatitis (NASH), and it is considered an independent risk factor for developing cardiovascular disease. The choline-deficient diet (CDD) causes changes in methionine metabolism and increases the concentration of homocysteine (Hcy) in blood and fat accumulation in the liver. Recent studies have shown that creatine supplementation can modulate the balance of transmethylation reactions and decrease the concentration of Hcy. However, the effects of creatine supplementation in models of CDD are little known. 
Objectives: To evaluate the effects of creatine supplementation on Hcy metabolism and fat accumulation in the liver induced by choline-deficient diet.

Methods: Separated into individual cages with controlled light and temperature, 24 rats (Wistar) were divided into three groups: control (C), choline-deficient diet (CDD) and choline-deficient supplemented with creatine (CR). The DDC was composed of control diet (AIN-93) exempts from the recommended amount of choline. Creatine supplementation was performed by adding $2 \%$ (weight / vol) in the diet of the group CR. The diets were given to animals for 28 days on an ad libitum.

Results: The CDD increased significantly $(\mathrm{p}<0.05)$ plasma homocysteine concentration $(50 \%)$ and total fat (38\%), triglycerides $(42 \%)$ and lipid peroxidation $(23 \%)$ in the liver as well as decrease the concentration of S-adenosylmethionine (SAM, 25\%). Creatine supplementation significantly increased plasma concentration (303\%) and liver creatine $(478 \%)$ and significantly decreased its endogenous synthesis $(80 \%$ share of kidney AGAT). This mechanism caused a reduction in plasma homocysteine levels $(11.2 \pm 0.7 \mathrm{C}, \mathrm{CDD} 16.9 \pm$ 1.3, Cr 10.1 \pm 0.6 , umol / L) and liver fat (76.9 \pm 4 C, 5, CDD 106.4 $\pm 12.3, \mathrm{Cr} 76.7 \pm 11.8 \mathrm{mg} / \mathrm{g}$ tissue). The determination of gene expression of enzymes involved in homocysteine metabolism and choline showed that creatine supplementation normalized the mRNA of the enzymes BHMT and GNMT decreased by CDD. However, it has been found restoring the concentration of SAM in the CR group. Also, there were no significant changes in the concentrations of cysteine, methionine and vitamin B12.

Conclusions: Creatine supplementation prevents the increase in Hcy and fat accumulation caused by CDD, probably by modulation of enzymes involved in homocysteine metabolism.

\section{P128 \\ Association of PPAR-Gamma2 Polymorphism on Lipid Profile in Berardinelli-Seip Syndrome}

Maria de Fátima Paiva Baracho, Lucia Dantas Leite, Sancha Helena Lima Vale, Camila Xavier Alves, Maria Gore tti Nascimento Santos, Cristina Moreno Farjado, Mario Hiroyuki Hirata, Jose Brandao-Neto

Federal University of Rio Grande Do Norte

Background: Berardinelli-Seip syndrome (BSS) is a rare autosomal recessive disorder characterized by near-total lack of adipose tissue, prominent muscularity, insulin resistance, diabetes mellitus, dyslipidemia, and other clinical manifestations. Nearly 300 cases among several ethnic groups have been described in the literature, with significant prevalence in Northeastern Brazil. PPAR $\alpha$ and PPAR $\gamma$ are crucial for lipid and glucose metabolism, respectively, and have important role in this syndrome.

Objectives: Although the presence of PPAR- $\gamma 2$ Pro12Ala polymorphism has not been associated with plasma lipids concentrations, we aimed to verify possible association between this polymorphism and lipid profile of BSS patients.

Methods: We investigated twenty-four patients with BSS, treated at the Endocrinology Ambulatory of Onofre Lopes University
Hospital. The project was approved by the Ethics Committee. Biochemical analyses (glucose, cholesterol, triglycerides, HDL-c, LDL-c and VLDL-c) were conducted with enzymatic-colorimetric methodology. DNA was extracted as previously described. Analysis of $P P A R-\gamma 2$ polymorphism was conducted using polymerase chain reaction (PCR). To analyze Pro12Ala polymorphism of the PPAR- $\gamma 2$ gene, amplification products were digested by PCR and visualized in $8 \%$ polyacrymalide gel. The Kruskall-Wallis and the Fisher's test were applied. The GraphPad Prism was used for statistical data analysis with significance level of $5 \%(\mathrm{p}<0.05)$.

Results: Among patients 37.5\% were males and $62.5 \%$ females, with median age of 18.5 years. All had normal BMI for age. Diabetes was present in $54.2 \%$ of patients. In relation to lipids, all subjects showed high levels of triglycerides. Cholesterol levels were altered in $70.8 \%$, HDL-c in $83.3 \%$, LDL-c in $58.3 \%$ and VLDL-c in $91.7 \%$ of the patients. The Pro/Pro genotype of the Pro12Ala polymorphism was found in $87.5 \%$ of patients and the Ala/Ala variant was not observed in any of them. A significant association was found between the Pro/Pro genotype and higher levels of triglycerides $(p=0.010)$, VLDL $(p=0.008)$ and glycemia $(p=0.045)$ among subjects.

Conclusions: There was an association between Pro12Ala polymorphism of the PPAR- $\gamma 2$ gene and glycemia was found how expected. Moreover, there also was significant association with higher triglycerides and VLDL-c levels, suggesting an interrelationship of these parameters in lipid metabolism.

Financial support: CNPQ (471795/2010-0).

\section{P129 \\ Aging and L-Arginine Chronic Administration Modulate BMP-9 Expression in Hepatic Tissue of Rats}

\author{
Mayra Domiciano, Maíra Bispo, \\ João Gilberto Kamia Menezes, Carolina Scatena Sena, \\ Rachel Souza Luiz, Lucas Carminati Pantaleão, \\ Camila Schelles, Thais Castro-Barbosa, Bruno Cogliati, \\ Maria Lucia Dagli, Camilo Lellis-Santos, \\ Maria Tereza Nunes, Silvana Bordin, Luciana Caperuto \\ UNIFESP - Campus Diadema/ICB - USP/FMVZ - USP
}

Background: BMPs are members of the TGF-beta superfamily, which regulates several biological processes, as morphogenesis and cell proliferation. BMP-9 is predominantly expressed in non-parenchymal liver cells and was identified as a regulator of glucose metabolism. Purified recombinant BMP-9 was able to enhance insulin action in skeletal muscle and adipocytes, and to inhibit hepatic glucose production. L-arginine is a conditionally essential amino acid with controversial effects related to glycemic metabolism. In rats, chronic oral supplementation of L-arginine (35mg/day for 30 days) induces insulin resistance, hyperinsulinemia, and increased levels of growth hormone $(\mathrm{GH})$. Aging is a model that exhibits insulin resistance and low levels of GH.

Objectives: The aim of this study was to assess BMP-9 expression in two models of insulin resistance with different levels of GH: aging (50 wk) and L-arginine chronic supplementation (A35). 
Methods: RT-PCR and Western Blotting were used to assess BMP-9 genic and proteic liver regulation in rats after chronic supplementation of L-arginine or aging. L-arginine was administered orally for 30 days. Effects of aging were evaluated in 50 weeks old rats. Insulin tolerance test (ITT) was performed to evaluate glycemic metabolism.

Results: There was no difference in growth rate between supplemented and control rats, which showed that L-arginine supplementation did not promote obesity. Rats became insulin resistant after L-arginine supplementation with $35 \mathrm{mg}(\mathrm{CTL}=4.04 \pm 0.24, \mathrm{~A} 35=$ $2.47 \pm 0.31, \mathrm{p}<0.05)$. Aging rats developed insulin resistance $(\mathrm{CTL}=$ $3.30 \pm 0.11,50 \mathrm{wk}=2.07 \pm 0.05, \mathrm{p}<0.05)$. There was no difference in BMP-9 genic expression after L-arginine supplementation. However, BMP-9 genic expression was decreased in aging rats $(\mathrm{CTL}=1.74 \pm$ $0.03,50 \mathrm{wk}=1.12 \pm 0.01, \mathrm{p}<0.05)$. Expression of unprocessed BMP-9 after L-arginine supplementation was not altered, while aging induced an increased expression of unprocessed BMP-9 $(\mathrm{CTL}=3085$ $\pm 557,50 \mathrm{wk}=11064 \pm 1624, \mathrm{p}<0.05)$. Mature BMP-9 was increased with $35 \mathrm{mg}$ supplementation $(\mathrm{CTL}=8207 \pm 621, \mathrm{~A} 35=11166 \pm 723$, $\mathrm{p}<0.05)$, as well as in aging $(\mathrm{CTL}=1126 \pm 105,50 \mathrm{wk}=2371 \pm 68$, $\mathrm{p}<0.05)$.

Conclusions: Regulation of genic and proteic expression of BMP-9 were different in L-arginine supplementation and aging rats. Both models show particular GH levels. This modulation of BMP-9 expression probably participates in the insulin resistance observed in these models.

Financial support: FAPESP / CNPQ (2009/50041-0 / 475091/2008 6).

\section{P130 \\ Nutrigenomics Approach in Children Nutritional Education}

\section{Luciana Pereira Souza, Nilce Naomi Hashimoto}

Universidade Cruzeiro do Sul

Background: Nutrigenomics studies the influence of nutrients in the expression of some genes responsible for the manifestation of some diseases and how these can be prevented through a healthy diet. Such prevention can be accomplished through effective actions in children nutritional education.

Objectives: Promote interactions between the new knowledge of Nutrigenomics and Children Nutritional Education, characterize the presence of non-communicable chronic diseases (NCDs) in students and their families, develop and evaluate didactic material for the prevention of NCDs since childhood, analyze the school snacks taken by students before and during nutritional intervention.

Methods: longitudinal and descriptive research in a public school with 32 children (7-8 years old) who had their school snacks analysed for sixty days. The observation, registered through a checklist, occurred at two different times, thirty days without nutritional intervention, and the following thirty days with nutritional intervention and the use of didactic material for the understanding of concepts about DNA, genetics, NCD and healthy feeding. There was a questionnaire to parents with closed questions about family history regarding NCDs and their opinion about the food offered at home and snacks taken at school. The data obtained was processed by the statistical analysis and presented in tables and graphs. The research was approved by the Ethics Committee, respecting the legal standards for studies with humans.

Results: All students had a relative suffering from an NCD, for example $65,63 \%$ have arterial hypertension and $21,88 \%$ obesity. About school snacks, $65,63 \%$ of parents say there are chosen by the children themselves and $37,50 \%$ consider them unhealthy. After the dietary interventions considerable improvement in the quality of school snacks was revealed, with reduced consumption of food with low nutritional value such as soda $(35,72 \%)$ and chips $(43,48 \%)$ and increased consumption of healthy food like bananas $(72,88 \%)$ and yoghurt $(49,16 \%)$.

Conclusions: During all the process the understanding of the new concepts was observed, such fact is confirmed by the change of behavior refleted in changes in the quality os school snacks and children's reports about their own history of NCDs in their families. Therefore, nutritional education based on Nutrigenomics can be effective even for children, if done by the appropriate techniques and didactic materials.

\section{P131 \\ Polymorphism in the Transcription Factor NRF2 Influence on Gene Expression of Antioxidant Enzymes}

\author{
Luciane Luca Alencar, Thomas Prates Ong, \\ Silvia M.F. Cozzolino \\ Universidade de São Paulo
}

The cellular enzymatic antioxidant defense system displays a major pathway, Nrf2/ARE (NF-E2 related factor $2 /$ antioxidant response element), which through transcription of Phase II enzymes such as glutathione peroxidase, glutathione transferase, catalase, quinone oxidoreductase 1 , heme oxygenase 1 , prevents cellular damage caused by reactive oxygen species (ROS). Thus, this study aims to review the influence of single nucleotide polymorphisms (SNP) in the gene that encodes the transcription factor Nrf2. Original articles (2005 to the present) have been reviewed in the pubmed databases. With genetic sequencing, it is possible to evaluate the responses resulting from genotype. Until now, nine SNPs were identified in the gene that encodes the transcription factor $\mathrm{Nrf2}$, and two of these, $-617 \mathrm{C} / \mathrm{A}$ and $-651 \mathrm{G} / \mathrm{A}$, are located in the promoter region and are related to the reduction of gene transcription of antioxidant enzymes. In humans, polymorphisms $-617 \mathrm{C} / \mathrm{A},-651 \mathrm{G} / \mathrm{A}, 336 \mathrm{~T} / \mathrm{C}$ have been associated with both a reduction in gene expression of enzymes involved in antioxidant defense system as well as with chronic diseases, such as diabetes mellitus, atherosclerosis, cancer and respiratory diseases. Currently it is known that there is an interaction between genes and other DNA sequences with environmental factors, and these interactions may influence gene expression and/or protein and its function. The presence of these polymorphisms has been associated with functional alterations of translated proteins. When relating the presence of polymorphisms $-617,-651$ and -653 with nutritional aspects, it was found that coffee consumption increased expression of Nrf2. Similarly, some medications, such as phenytoin, 
also increased the gene expression of the transcription factor Nrf2 in the presence of polymorphisms-3089G/A. Thus, Nrf2 represent and im.

\section{P132}

\section{Macadamia Oil Prevents the Increase in the Expression of Genes Related to Muscle Proteolytic Degradation in a Diet-Induced Obesity Murine Model: Role of E3 Ubiquitin Enzyme Ligases, MURF-1 and ATROGIN-1}

\author{
Luís Gustavo de Sousa, Carlos Flores Rodrigues Jr. \\ José Cesar Rosa Neto, Alice Cristina Rodrigues, \\ Edson Lima, Luiz Roberto Grassmann Bechara, \\ Patricia Chakur Brum, Tania Pithon-Curi, Rui Curi, \\ Sandro Massao Hirabara \\ Institute of Biomedical Sciences
}

Background: Increased activity of the ubiquitin proteasome system (UPS) has been observed on skeletal muscle in several murine models of obesity. This increase can be mediated by elevated expression of the E3 ubiquitin enzyme ligases, MuRF-1 and atrogin-1/ MAFbx. Macadamia oil is rich in omega-7 polyunsaturated fatty acid (n-7 PUFA), which is associated with decreased inflammatory process and improved lipid profile. However, effect of macadamia oil on regulation of protein degradation pathway on skeletal muscle has not been investigated yet.

Objectives: The aim of the present study was to assess the effect of macadamia oil on expression of genes involved in proteolytic pathways (Murf-1 and Atrogin-1) in diet-induced obese C57/BL6J mice.

Methods: C57BL/6J mice were fed a control or a high-fat diet and supplemented or not with macadamia oil $(2.5 \mathrm{mg} / \mathrm{kg})$ for eight weeks. mRNA levels of E3 ubiquitin enzyme ligases, MuRF-1 and atrogin-1/ MAFbx, were evaluated in EDL muscle by real-time PCR.

Results: Mice fed a high-fat diet presented decreased weight of EDL muscle when compared to control diet (0.2783 vs 0.3940 , respectively), suggesting skeletal muscle atrophy. Supplementation with macadamia oil prevented this effect $(0.2860$ vs control 0.3483 , $\mathrm{p}<0.05$ ). In order to evaluate skeletal muscle proteolytic systems, we measured MuRF1 and Atrogin-1/MAFbx mRNA levels, in EDL muscle from high-fat diet or control mice, treated or not with macadamia oil. We observed a significant two-fold increase in mRNA levels of E3 ubiquitin enzyme ligases, MuRF-1 and atrogin-1/ MAFbx, in EDL muscles from mice fed a high-fat diet compared to mice fed a control diet. On the other hand, supplementation with macadamia oil restored mRNA levels of Murf-1 and Atrogin-1 in mice fed a high-fat diet to control levels, suggesting a protection of macadamia oil on the pathways involved in the diet-induced proteolysis.

Conclusions: Our data suggest that the increase in skeletal muscle degradation in obesity is associated with elevated expression of genes involved in muscle proteolysis, which is prevented by macadamia oil supplementation.

Financial support: FAPESP (2010/18921-7).

\section{P133}

\section{Antioxidant Capacity of Dietary Polyphenols and their Impact on Gene Expression in CACO2 Cell Lines}

\author{
Luiza Nicolosi Guido, Aïchatou Dior, Abalo Chango, \\ Thais M.F.S. Vieira
}

Institut Politechnique Lasalle Beauvais - France

Colon cancer is the third most common type of cancer and second leading cause of death worldwide. Among the risk factors for developing colon cancer, diet is one of the most important. In the last decades, the link between nutrition and health began to be studied at a genetic and epigenetic level. The nutrients presents in food can influence on genetic and epigenetic process that occur during life altering gene expression and cell multiplication, survival and differentiation. Thus, some nutrients can show a harmful effect for health, leading to the development of diseases or a benefic effect, protecting the organism against malignant cell transformation. Phenolic compounds presents in plants are responsible for protection against oxidative stress, pests and predators. Besides that, in human, they have shown anti inflammatory, antioxidant and anticancer properties. Recent evidences have demonstrated that phenolic compounds present in plants also influence on epigenetic alterations. This study relates the effect of phenolic compounds extract from Sorghum and Grape on $\mathrm{CaCo} 2$ cell line, based on nutrigenomics approaches, gene expression and epigenetic process. The phenolic compounds were extracted from Sorghum and Grape, with quantification and determination of antioxidant power. Cell line $\mathrm{CaCo} 2$ was treated with phenolic compounds at $40 \mathrm{mM}$ and protection against oxidative stress by lipid oxidation, evaluation of the effect on gene expression and on DNA structure were measured. The phenolic compounds found in Sorghum and Grapes have shown an antioxidant activity, protecting cells against oxidative stress during their life cycle, by inhibiting lipid oxidation. The compounds also had effect on the expression of genes related to epigenetic alterations, as DNMTs, MBD2 and GAMT, all related to DNA methylation, and presented activity on DNA structure, providing an increase on chromatin accessibility and decreasing the level of gene silencing of tumor suppressor gene as P16IK4a, related to cell cycle control. The present results show that the treatment of $\mathrm{CaCo} 2$ cells with phenolic compounds from Sorghum and Grape are related to the cell protection against oxidative stress, damage and epigenetic alterations, altering the expression of genes related with epigenetic alterations and cell cycle control, which could be protective for cell differentiation, avoiding malignant transformation. 
P134

Associations of the TNF-Alpha -308G/A, IL6 -174G/C and ADIPOQ 45T/G Polymorphisms with Inflammatory and Metabolic Responses to Lifestyle Intervention in Brazilians at High Cardiometabolic Risk

Maira Ladeia Rod Curti, Milena Monfort Pires, Camila Riso Barros, Antonela Siqueira-Catani, Sandra Roberta G. Ferreira

Department of Nutrition, School of Public Health, University of Sao Paulo, Brazil

Background: Cytokines secreted by the adipose tissue influence inflammation and insulin sensitivity, and lead to metabolic disturbances. How certain single-nucleotide polymorphisms (SNPs) interfere on lifestyle interventions is unclear. We assessed associations of selected SNPs with changes induced by a lifestyle intervention.

Methods: This 9-month intervention on diet and physical activity included 180 Brazilians at high cardiometabolic risk, genotyped for the TNF- $\alpha-308 \mathrm{G} / \mathrm{A}$, IL-6 -174G/C and AdipoQ 45T/G SNPs. Changes in metabolic and inflammatory variables were analyzed according to these SNPs. Individuals with at least one variant allele were grouped and compared with those with the reference genotype.

Results: In the entire sample $(66.7 \%$ women, mean age $56.5 \pm$ 11.6 years), intervention resulted in lower energy intake, higher physical activity, and improvement in anthropometry, plasma glucose, HOMA-IR, lipid profile and inflammatory markers, except for IL-6 concentrations. After intervention, variant allele carriers of the TNF- $\alpha$ -308G/A decreased plasma glucose. Regarding the IL6 -174G/C SNP, carriers of the variant allele had a better response of lipid profile and adiponectin concentration, but only the reference genotype group decreased plasma glucose. In contrast to individuals with the reference genotype, carriers of variant allele of AdipoQ 45T/G SNP did not change plasma glucose, apolipoprotein B, HDL-c and adiponectin concentrations in response to intervention.

Conclusion: The TNF $\alpha-308 \mathrm{G} / \mathrm{A}$ SNP may predispose a better response of glucose metabolism to lifestyle intervention. The IL-6 -174 G/C SNP may confer a beneficial effect on lipid but not on glucose metabolism. Our findings reinforce unfavorable effects of the AdipoQ 45T/G SNP in lipid profile and glucose metabolism after intervention in Brazilians at cardiometabolic risk. Further studies are needed to direct lifestyle intervention to subsets of individuals at cardiometabolic risk.

Financial support: FAPESP.

\section{P135 \\ Indicated for the Young Investigator Award \\ FTO T/A, PPARgamma PRO12ALA but not APOA1-75 Polymorphisms are Associated with Better Response to Lifestyle Intervention in Brazilians at High Cardiometabolic Risk}

\author{
Maíra Ladeia R. Curti, Marcelo Macedo Rogero, \\ Camila Riso Barros, Antonela Siqueira-Catani, \\ Valéria Troncoso Baltar, Sandra Roberta G. Ferreira \\ School of Public Health, University of Sao Paulo
}

Background: The role of obesity-related polymorphisms on weight loss and inflammatory responses to interventions is unclear. We investigated associations of certain polymorphisms with response to a lifestyle intervention.

Methods: This 9-month intervention on diet and physical activity included 180 Brazilians at high cardiometabolic risk, genotyped for the FTO T/A (rs 9939609), PPAR $\gamma$ Pro12Ala (rs 1801282) and Apo A1-75G/A (rs 670) polymorphisms. Changes in metabolic and inflammatory variables were analyzed according to them, adjusted for age, gender, antihypertensive and lipid-lowering medications.

Results: The intervention resulted in lower energy intake and higher physical activity. Anthropometric measurements, 2-h plasma glucose, insulin, HDL-c and Apo B improved significantly for total sample and these benefits did not depend on the genotypes. Only variant allele carriers of FTO T/A decreased fasting plasma glucose after intervention $(100.0 \pm 1.3$ to $95.6 \pm 1.4 \mathrm{mg} / \mathrm{dL}, \mathrm{p}=0.021)$. Mean blood pressure reduced after intervention in variant allele carriers of the PPAR $\gamma$ Pro12Ala $(109.4 \pm 2.1$ to $101.3 \pm 2.1 \mathrm{mmHg}, \mathrm{p}<0.001)$. Improvement in lipid variables was not significant after adjustment for medication. Only the reference genotype of PPAR $\gamma$ Pro12Ala increased Apo A1 after intervention $(134.3 \pm 2.4$ to $140.6 \pm 2.3 \mathrm{mg} /$ $\mathrm{dL}, \mathrm{p}<0.001)$. Only variant allele carriers of FTO reduced CRP concentration $(0.366 \pm 0.031$ to $0.286 \pm 0.029 \mathrm{mg} / \mathrm{dL}, \mathrm{p}=0.023)$.

Conclusion: In Brazilian individuals, the FTO T/A induces a favorable impact on inflammatory status and glucose metabolism. The reference genotype of PPAR $\gamma$ Pro12Ala seems to favor a better lipid profile, while the variant allele decreases blood pressure. Our data did not support benefits of the variant allele of Apo A1 -75G/A polymorphism. Further studies are needed to direct lifestyle intervention to subsets of individuals at cardiometabolic risk.

Financial support: FAPESP. 


\section{P136}

\section{Hepcidin Expression and Oxidative Stress in Diabetic and Diabetic Iron-Supplemented Rat Liver}

\author{
Maisa Silva, Joyce Ferreira Costa Guerra, \\ Marcelo Estáquio Silva, Maria Lúcia Pedrosa
}

Universidade Federal de Ouro Preto

Increased oxidative stress is widely accepted as a participant in the development and progression of diabetes and its complications. A range of biochemical disturbances may result from dysregulated iron metabolism, including metabolic disorders affecting glucose and insulin that lead to diabetes. Moreover, diabetes mellitus is associated with altered iron homeostasis in both human and animal diabetic models. Accumulating evidence suggests that dysregulation of hepcidin expression is one of the primary causes of hepatic iron overload. Thus, we investigated whether the expression of liver hepcidin is altered in diabetes, as this could potentially affect oxidative stress. Rats were divided into four groups: rats in the control (C) group received a standard AIN-93 diet, rats in the control iron (CI) group received a standard diet supplemented with $0.83 \%$ carbonyl iron, rats in the diabetic (D) group received a single injection of streptozotocin $(\mathrm{STZ}, 35 \mathrm{mg} / \mathrm{Kg}$ ) on day 15 , and rats in the diabetic iron (DI) group were streptozotocin treated animals that received a diet supplemented with $0.83 \%$ carbonyl iron. The diets were given for 45 days. Animals from the D $(62.41 \pm 23.9 \mu \mathrm{mol} / \mathrm{L}), \mathrm{CI}(92.86 \pm 26.8 \mu \mathrm{mol} / \mathrm{L})$ and DI $(70.32 \pm 23.4 \mu \mathrm{mol} / \mathrm{L})$ group showed higher levels of serum iron compared with C $(24.71 \pm 7.08 \mu \mathrm{mol} / \mathrm{L})$. Diabetes $(9.58 \pm 1.2 \mathrm{U} / \mathrm{mg}$ protein) and diabetes iron $(8.74 \pm 1.22 \mathrm{U} / \mathrm{mg}$ protein) rats presented an increased concentration of carbonyl protein liver in relation to the group control ( $4.38 \pm 0.46 \mathrm{U} / \mathrm{mg}$ protein). TBARs levels in liver were increased in groups receiving iron overload associated with diabetes or not $(83 \%$ and $71 \%$ respectively). Quantitative PCR analysis showed increased expression of hepcidin in $81 \%$ higher in DI rats than in control rats. The results presented here suggest that diabetes associated or not with iron can cause perturbations in iron status and oxidative stress.

Financial support: CNPQ, CAPES, FAPEMIG, UFOP.

\section{P137 \\ Hypercholesteloremic Diet Increases Expression of the NADPH Oxidase Subunit p22PHOX in Rat Liver}

\author{
Isabel C.M.E. de Abreu, Maisa Silva, Joyce F.C. Guerra, \\ Marcelo Eustaquio Silva, Maria Lucia Pedrosa \\ Universidade Federal de Ouro Preto
}

Hypercholesteloremic diet induces oxidative stress. Reactive oxygen species (ROS) may be generated by multiple sources including NADPH oxidases. The NADPH oxidases are a group of plasma membrane associated enzymes found in a variety of cells of mesodermal origin. It catalyzes the production of superoxide (O2-) by the one electron reduction of oxygen, using NADPH as the electron donor.
Activation of the NADPH oxidase requires the targeting of a cytoplasmic p40-p47-p67(phox) complex to the membrane bound heterodimeric p22-gp91(phox) flavocytochrome. The p22 PHOX subunit is an essential component of the NADPH oxidase, which is considered the major source of oxidative stress products. This way, an increase in an expression of NADPH subunit p22 PHOX can suggest an increase in a production of ROS. The current study aimed to investigate if an increase in oxidative stress generated by hypercolesterolemic diet was associated with changes in the expression of NADPH oxidase subunit 22PHOX in a rat model. Female rats were divided into two groups: Control (C), which received a standard AIN 93 diet and Hypercolesterolemic $(\mathrm{H})$, composed of animals that received a hypercholesterolemic diet with $25 \%$ of soybean oil and $1 \%$ cholesterol. The diets were given for 56 days. Quantitative PCR analysis showed increased expression of NADPH oxidase subunit p22PHOX in hypercolesterolemic rats compared to the control group $(\mathrm{p}=0.006)$. The result presented here suggests that hypercolesterolemic diet induces oxidative stress by NADPH oxidase increasing the amount of superoxide anion available.

Financial support: CNPQ, CAPES, FAPEMIG, REUNI, UFOP.

\section{P138 \\ Phenolic Compounds of Jaboticaba (Myrciaria Jaboticaba) Improve Insulin Sensitivity and Glucose Tolerance in Obese Rats}

Marcela Roquim Alezandro, William Tadeu Lara Festuccia, André Marette, Franco Maria Lajolo, Maria Inés Genovese

\section{Universidade de São Paulo}

Strong evidences indicate that dietary polyphenols influence carbohydrate metabolism through several mechanisms including inhibition of carbohydrate digestion and glucose absorption in the intestine, stimulation of insulin secretion from the pancreatic beta-cells, modulation of hepatic glucose production, activation of insulin receptors and glucose uptake in the insulin responsive-tissues, and modulation of intracellular signaling pathways and gene expression. To test whether polyphenols have beneficial actions in the prevention of dietinduced insulin resistance and type 2 diabetes, C57BL/6J mice fed with a high-fat/high sucrose (HF/HS) diet were treated by oral daily gavage with water or polyphenols from jaboticaba purified in PA or C18 for 8 weeks. Mice were evaluated for glucose and insulin tolerances (GTT and ITT, respectively), antioxidant capacity, lipid profile, glycemia and hepatic and renal function (urea, creatinine, AST (aspartate aminotransferase) and ALT (alanine aminotransferase)). Simultaneously, L6 myocytes were treated with crude extract (CE) and the purified fractions in polyamide (PA) and $\mathrm{C} 18$ resin, in the concentrations of $0.5,5.0$ and $50.0 \mathrm{ppm}$, with and without insulin stimulation. In mice, polyphenols from jaboticaba improved glucose tolerance and insulin sensitivity, without affecting body weight gain or food intake. Mice treatment with jaboticaba polyphenols significantly reduced plasma triacylglycerol, but it did not affect other circulating lipids or hepatic and renal functions. In L6 myotubes, the treatment with jaboticaba extract increased glucose uptake, especially the lowest dose $(0.5 \mathrm{ppm})$. This effect was observed in the three dif- 
ferent extracts and doses. In the absence of insulin, there was an increase of $76 \%$ for CE, $114 \%$ for PA and $82 \%$ for C18. In the presence of insulin, glucose uptake was raised in $73 \%$ for $\mathrm{CE}, 71 \%$ for PA and $57 \%$ for $\mathrm{C} 18$. In conclusion, polyphenols from jaboticaba protected mice from the development of glucose intolerance and insulin resistance induced by a HF/HS diet, such an effect that may involve an improvement in basal and insulin stimulated glucose uptake in skeletal muscle.

Financial support: FAPESP (2009/01775-0).

P139

Polyphenols Content and Antioxidant Activity of Cooking Spices Curcuma, Curry Powder, Black Pepper and Hot Paprika

Marcelo Chaves de Azevedo, Christiane Queiroz, Renata Madureira Polinati, Eliane Fialho

Universidade Federal do Rio De Janeiro

Background: Cooking spices such as curcuma, curry powder, black pepper and red pepper have been casting on the kitchen for a long time because of their food coloring and flavoring properties. However, these spices go further beyond the kitchen and present a great deal of antioxidant and anti-inflammatory activities due to the role of their bioactive compounds curcumin, piperine and capsaicin.

Objectives: To determine the polyphenols content and the antioxidant activity of three different brands of each curcuma, curry powder, black pepper and hot paprika available at the local market.

Methods: Soluble (SP) and hydrolysable (HP) polyphenols content was determined by the method of Folin-Ciocalteu reagent and the results were expressed in $\mathrm{mg}$ of galic acid equivalent $(\mathrm{mgGAE} / \mathrm{g})$. The antioxidant activity was determined by the methods of DPPH which was expressed in \% of inhibition and FRAP results were expressed in trolox equivalent millimolar (mM ETX).

Results: The polyphenols content in curcuma ranged from 1.4 to $1.8 \mathrm{mgGAE} / \mathrm{g}$ (SP) and 2.7 to $3.7 \mathrm{mgGAE} / \mathrm{g}(\mathrm{HP})$, in curry powder it ranged from 1.5 to $1.8 \mathrm{mgGAE} / \mathrm{g}$ (SP) and 2.7 to $3.7 \mathrm{mgGAE} / \mathrm{g}(\mathrm{HP})$, in black pepper it ranged from 1.7 to $2,1 \mathrm{mgGAE} / \mathrm{g}(\mathrm{SP})$ and 3.5 to $3.6 \mathrm{mgGAE} / \mathrm{g}(\mathrm{HP})$ and in hot paprika it did range from 1.5 to 2.2 $\mathrm{mgGAE} / \mathrm{g}$ (SP) and 2.5 to $3.7 \mathrm{mgGAE} / \mathrm{g}$ (HP). The HP extracts presented the highest antioxidant activity, being curcuma $65 \%$, curry powder $67 \%$, black pepper $64 \%$ and hot paprika $62 \%$ DPPH inhibition. FRAP results also showed the HP extracts with the highest antioxidant activity, being curcuma $13 \mathrm{mM}$ ETX, curry powder $14 \mathrm{mM}$ ETX, black pepper $11 \mathrm{mM}$ ETX and hot paprika $6 \mathrm{mM}$ ETX.

Conclusions: The four cooking spices turned out to present a satisfactory amount of polyphenols and antioxidant activity enough to be considered as an effective health promoting tool available on a daily basis.

\section{P140}

\section{Nutrigenomics: Influences of Genetic Variation and Nutrition in Obesity}

\author{
Márcia Fábia Andrade Santos, Luciana Yuki Tomita \\ UNIP
}

The pandemic of obesity has generated a term established by WHO to define this situation: "globesity". With the completion of the Genome Project new areas were created in Nutrition: Nutrigenomics and the nutrigenetics, suggesting customized diets based on individual genotype. The strongest predictor gene associated with obesity, known so far is the gene FTO. Its variants are the most important genetic determinants of obesity. Of SNP's that were reported and were associated with obesity and BMI, the SNP rs9939609 is of particular interest. It is formed by the alleles A > T, and the A allele is considered hazardous and is associated with a higher fat accumulation, mainly in the homozygote form. Studies show the influence of the FTO gene on weight gain, although the mechanism for fat accumulation is unknown. However, there is an influence of environmental factors. For populations with high rates of physical activity, or who have lived in less obesogenic environments, or who have had food insecurity there was no risk for overweight and obesity. The studies investigated the relationship of FTO with chronic diseases, but there were no associations. Chronic diseases appear to be associated with excess body fat. The SNP's list of the FTO gene with obesity and its association with appetite suggest the presence of an important interaction between genes and nutrients. In conclusion based on the studies presented, the homozygotes had the worst food choices. This allele has been associated with an impaired capacity to satiety response. In relation to physical activity, the results found that the association of genotype on the body composition is much smaller and less statistically significant in subjects who have higher levels of physical activity.The association of the FTO gene with human obesity is well established in Europeans. A convergence of evidence indicates that obesity has strong influence of lifestyle and genetics, which may favor weight gain in patients with the risk allele, which seem to have choices for energy-dense foods and lower response to satiety. In the near future it may be useful to know if this is the individual risk allele, to determine the most effective way to develop a personalized eating plan with the genotype for the reduction of chronic diseases such as obesity. However more studies are needed to clarify the genetic influence on weight gain in different ethnic groups. 


\section{P141 \\ Effect of Tributyrin on the Skeletal Muscle Regeration and Hypertrophy}

Vitor da Silveira Alves, Diogo Vasconcelos,

Phablo Savio Teixeira, Carlos Hermano Pinheiro,

Luís G.O. de Sousa, Lucas Guimarães Ferreira, Rui Curi,

Marco Aurélio Ramirez Vinolo

Institute of Biomedical Sciences

Tributyrin (Tb) is a pro-drug of butyrate, a short chain fatty acid that, through inhibition of histone desacetylase (HDAC) activity, modulates inflammation, cell proliferation, differentiation and apoptosis. Regarding skeletal muscle cells, previous studies have shown that inhibition of HDAC activity increase MyoD acetylation and myoblast differentiation in miotubes. Therefore, the aim of the present study was to analyse the effect of oral administration of $\mathrm{Tb}$ on the skeletal muscle healing and hypertrophy. Male Wistar adult rats were treated dailly with $\mathrm{Tb}(2 \mathrm{~g} / \mathrm{kg})$ by gavage. Animals were pre-treated during one week and then submitted to the surgical procedures. The treatment persisted until the end of the protocol. Two protocols were used: in the model of hypertrophy, the muscle extensor tibialis anterior (ETL) was removed. Other group of animals had their tibialis anterior muscle lacerated (model of lesion). In both cases, the other leg (contralateral leg) was used as control. After 28 days, the animals were sacrificed and had their muscles functionally and histologycally analysed. Administration of $\mathrm{Tb}$ has only a slight effect on the recover of muscle strenght after lesion. However, a clear improvement was observed in the model of hipertrophy: a increase of more than $50 \%$ and $36 \%$ for isotonic and tetanic muscle forces was present in the animals treated with $\mathrm{Tb}$ compared with their control. As expected, an increase of EDL (extensor digitorum longus) weight was observed in the ETL ablated legs compared with the contralateral leg. However, no change was found when comparing the muscles of treated and non-treated rats. Histologycally, an increase in fibers diameters was found in animals that received $\mathrm{Tb}$. The results obtained herein indicate that $\mathrm{Tb}$ administration improve muscle hypertrophy in rats, an effect that may be dependent on the inhibition of HDAC activity.

Financial support: FAPESP (2011/12089-0).

\section{P142 \\ SNP 45TG Polymorphism in Macrosomic Children}

\author{
Silleno José Dias Júnior, Ana Luisa Silva Rodrigues, \\ Caroline Kroll, Sandra Ana Czarnobay, \\ Silmara Salete B.S. Mastroeni, Marco Fabio Mastroeni \\ University of Joinville Region - UNIVILLE
}

Background: Fetal macrosomia is defined as birth weight equal or greater than $4.000 \mathrm{~g}$, regardless of gestational age. The risk for macrosomia increases with maternal weight and height. The serum concentration of adiponectin, an adipocytokine released from adipose tissue, is inversely correlated with insulin resistance and is consequently reduced in obesity and type 2 diabetes mellitus. It is therefore possible that maternal serum adiponectin in early pregnancy may be a biomarker of the common metabolic derangement observed in obesity and GDM causing fetal macrosomia. Some studies indicate that a mutation in the gene SNP45TG is associated with changes in the expression of this cytokine, causing metabolic disorders. There are no studies that investigated the association between the gene SNP45TG and macrosomia.

Objectives: This study aimed to describe the potential of SNP 45TG polymorphism in the prediction of fetal macrosomia.

Methods: The sample was drawn from a larger study that investigated 435 pregnant women aged 18 years or over, gestational age 37-42 weeks and live births. For this study we considered only 24 macrosomic children. Genomic DNA was extracted from venous blood samples stored in the card "clonesaver," according to the manufacturer's instructions. Gene amplification $S N P 45 T G$ was performed by applying the PCR-RFLP techniques. Amplicons were digested by endonucleases and SmaI and PCR products were subjected to electrophoresis in submerged gel 1,5\% agarose.

Results: Regarding the genotype frequencies, genotype TT was the most prevalent $(83.3 \%)$, and the prevalence of TG genotype was $16.7 \%$. There were no cases of children with GG genotype. The allele frequency of allele " $G$ " was 0.92 , and allele " $A$ " was 0.08 .

Conclusions: The low frequency of allele " $G$ " found in this study suggests that the allele "G" does not influence the development of the children with macrossomia. Further tests involving serum adiponectin of mothers will still be made to complete the study.

Financial support: Fund to support research - UNIVILLE.

\section{P143 \\ BIP Attenuates Endoplasmic Reticulum Stress Induced by Fatty Acids}

Maria Anete Santana Valente, Patrícia A. Fontes Vieira, Meire Oliveira Barbosa, Gabrielle Luck Araujo

UFMG/FAMINAS/UFV/UFLA

The endoplasmic reticulum (ER) is the first compartment of the secretory pathway of eucaryotic exerting important functions, such as synthesis and modification of proteins and lipid biosynthesis. BiP (binding protein) is a multifunctional protein involved in regulating cellular processes associated with the endoplasmic reticulum. The overload of cholesterol in the human body can affect the homeostasis of the ER, and consequently predispose to organelle stress that subsequently leads to accumulation of poorly folded proteins in the lumen of the ER. High levels of free fatty acids are also associated with dysfunction and apoptosis in adipose tissue is not a mechanism known as lipotoxicity. The accumulation of entrapped proteins incorrectly leads to activation of the response to poorly folded proteins, called the UPR pathway, which includes adaptive mechanisms to restore the normal functions of RE. However, prolonged and intense stress of the ER triggers cell death, usually in the form of apoptosis. Deleterious effects on cell viability due to stress of the ER have also been correlated with high levels of free fatty acids, particularly saturated fatty acids (such as palmitic acid) into cells of various tissues, including cardiac cells, pancreatic cells, adipocytes and hepatocytes. Recent investigations have centered on the stress of the ER as a 
potential mediator of apoptosis induced by high levels of fatty acids. However, the mechanisms are not fully understood. Obesity is associated with the pathophysiology of the ER stress, which plays a role in cardiac dysfunction in obese patients. Liver cells treated with saturated fatty acids showed reduced levels of BiP protein expression, whereas cells treated with unsaturated fatty acid did not change expression of this protein. It is known that overexpression of the protein BiP mitigates stress of the ER-induced free fatty acids and leads to a significant reduction in fatty acid-mediated apoptosis, suggesting a correlation between stress of the ER and lipotoxicity in liver cells. According to these data, it is important to emphasize the importance of balance of power, especially in regards to be saturated fatty acids in the control of obesity and chronic degenerative diseases.

\section{P144}

The Protective Role of Selenium and Vitamins C and E Against Cisplatin-Induced Nephrotoxicity

\author{
Maria Anete Santana Valente, Gabrielle Luck Araujo, \\ Patricia A. Fontes Vieira, Carlos Alberto Tagliati \\ UFMG
}

Cisplatin (cis-diamminedichloroplatinum II) is an effective chemotherapeutic agent used in the treatment of neoplasms. Despite its effectiveness as an antitumor drug, nephrotoxic side effects have significantly restricted its clinical use. Although prophylactic measures are widely used, such as moisture and intensive forced diuresis, irreversible renal damage occurs in about one third of patients treated with this drug. Research suggests that one of the main mechanisms of nephrotoxicity caused by cisplatin are renal changes of mitochondria and reactive oxygen species (ROS), substances potentially harmful to the body. Although mitochondria contain an antioxidant defense system, also seems to be the main source of ROS such as superoxide and hydroxyl radicals. In most cases, as an aggravating factor, the patient under treatment presents malnourished and with a poor diet, lacking in reserves and intake of vitamins and minerals, which decreases the antioxidant defense mechanisms and promotes the installation of renal oxidative stress, with the formation of ROS. The chemical reactivity of ROS with other molecules is very high, can cause lipid peroxidation, oxidation of nucleic acids and proteins and carbohydrates. The lipid peroxidation promotes, for example, the destruction of cell membranes. Recently, strategies are being developed for control of the antioxidant system of mitochondria with the purpose of this organelle reducing injuries due to poisoning caused by oxidative stress. Control of gene expression glutathione peroxidase and catalase are important for the ROS produced by the cellular metabolism are maintained in low concentrations. In investigations on the modulation of antioxidant power in the antitumor cisplatin nephrotoxicity, there are the vitamins C, E and Selenium. These antioxidants in the diet showed protection on the biomarkers nephrotoxicity, as in the expression of catalase and glutathione reductase. Thus, it is important to highlight the protective role of these nutrients, in patients receiving chemotherapy with cisplatin, against oxidative stress and subsequent renal toxicity.

\section{P145}

\section{Association Between the Leptin in Maternal Blood and Placenta and the New Born's Weight}

\author{
Maria Carmen Neves Souza Carmo, \\ Solange Silveira Pereira, Danielle Fernandes Durso, \\ Raquel Neves Ruas, Jacqueline Alvarez Leite
}

Universidade Federal de Minas Gerais

Leptin is a hormone secreted by white adipose tissue, mainly subcutaneous tissue. Blood leptin levels and adipose tissue mass are positively related. Leptin is an afferent signal of satiety, suppressing food intake and stimulating energy expenditure. Leptin is mainly expressed in adipose tissue. However, it can also be found in other tissues such as ovaries, placenta, brown adipose tissue, and bones. Leptin levels are higher in women than in men and even higher during pregnancy. Leptin levels in maternal blood begin to rise early in pregnancy and return to pre pregnancy levels 24 hours after delivery. Leptin levels are significantly higher in umbilical vein than in umbilical arteries. Although the regulation of maternal and fetal weight during pregnancy is not yet clear, it is possible that leptin has a role as a regulating factor. Evidences strongly suggest that placenta is an important source of leptin during pregnancy. The goal of this study was to investigate if the leptin levels in maternal blood and in umbilical vein and arteries and the expression of ob gene in placenta are related with new borns' weight in normal risk pregnancies (without presence of diabetes, pre eclampsia, obesity, etc.). This study used the data of 27 patients. We collected samples of maternal blood in a peripheral vein, blood from umbilical vein and arteries and samples of placental tissue right after delivery. We found a positive relation between maternal leptin levels and gain of weight by the mother. The data also showed a negative relation between maternal leptin levels and the new born's weight.

\section{P146 \\ Antioxidant Potential of Unpublished Xylofuranoside Front to Mn Toxicity in C. Elegans}

Willian Goulart Salgueiro, Suzi Giliane Wollenhaupt, Ana Thalita Gonçalves Soares, Maria Eduarda Lima, Daniela Freitas Câmara, Daiandra Fagundez, Daiana Silva Avila

Universidade Federal do Pampa

Introduction: The nematode C. elegans has been widely used due to it is a low cost experimental model with great possibilities in the area of molecular biology because its genome is completely known. It is transparent and has a small life cycle, large reproductive capacity and developments quickly. Organochalcogens have been widely studied due to their well-known antioxidant potential, they may provide neuroprotection, antiulcer and antidiabetic properties, which are closely related to their antioxidant activity. Manganese $(\mathrm{Mn})$, an abundant element in the crust, is necessary for reproduction 
regulation and normal brain function, being required for activation of many enzymes. Nevertheless, continuous exposure to Mn can lead to cell death due to the activation of necrosis and/or apoptosis pathways.

Objectives: Determine whether new organochalcogens belonging to the xylofuranosides class can protect against $\mathrm{Mn}$-induced toxicity in C.elegans and also determine whether the levels of Mn absorbed by the nematode are reduced by these compounds.

Methodology: For survival assay, 2500 synchronized L1 worms were divided into three groups: 1) tellurium xylofuranoside only, 2) with $\mathrm{MnCl}_{2}$ only and 3) $\mathrm{MnCl}_{2}$ and followed by the xylofuranoside. The worms were treated for $30 \mathrm{~min}$ and after 3 washes transferred to recovery plates containing E. coli OP50, being scored or survival after 24h. To measure Mn levels, $10000 \mathrm{~L} 1$ worms were pre-treated with $\mathrm{MnCl}_{2}$, washed three times with $\mathrm{NaCl} 0.5 \%$, and treated with the compound. After eight washes with $\mathrm{NaCl} 0.5 \%$ samples were stored at $20^{\circ} \mathrm{C}$. Mn levels were measured with a graphite furnace atomic absorption spectrophotometry. Statistical analysis was performed one-way ANOVA and Tukey post hoc. P values of $<0.05$ were considered statistically significant.

Results: In the survival assay, no tested concentration of tellurium xylofuranoside was capable of reversing the damage induced by $\mathrm{Mn}$. Mn levels of treated groups was increased dramatically, but the tellurium compound showed no significant capacity to decrease the absorption of the metal in the nematode.

Conclusions: It is known that $\mathrm{Mn}$ is absorbed by the worm through divalent metal transporters and thus, continuous exposure to Mn can cause toxicity to the worm, as observed by decreased survival followed Mn exposure. The tellurium xylofuranoside did not seem to compete with $\mathrm{Mn}$ for the same transporters, not capturing or even reversing the damage caused by this metal.

\section{P147 \\ Evaluation of Extracts of Ilex Paraguariensis on Survival, Reproduction and Stress Resistance with Paraquat in Caenorhabditis Elegans}

\author{
Maria Eduarda Lima, Ana Ceolin Colpo, \\ Felipa Melgarecho Bassante, Willian Goulart Salgueiro, \\ Hemerson Silva Rosa, Caroline Silveira Martinez, \\ Daiana Silva Avila, Vanderlei Folmer
}

Universidade Federal do Pampa

Background: Ilex paraguariensis, popularly known as yerbamate, is a native specie of tree in South America, used to prepare a peculiar drink. Recent studies have shown that phenolic compounds presented in yerba mate have antioxidant properties. Caenorhabiditis elegans a free-living nematode found in the liquid portion of the soil and is considered a suitable model to study aging and longevity because it has short life cycle, large reproductive capacity and rapid development.

Objectives: This study investigated the effect of acute exposure to extracts of different brands of yerba mate (I. paraguariensis) from Brazil on the survival, reproduction and stress resistance in C. elegans.
Methods: The herbal compounds were purchased from a commercial establishment in Brazil. The brands analyzed weres: Barão, Madrugada and Vier. The aqueous extracts were obtained mimicking the "mate" preparation, by using a medium size gourd, yerba mate, "mate pump", Kitasato flask and a vacuum pump. The extracts of the $1^{\text {st }}, 2^{\text {nd }}, 5^{\text {th }}, 10^{\text {th }}, 15^{\text {th }}$ infusions were filtered and stored forsubsequent experiments. Assays of survival, reproduction and oxidative stress with Paraquat in C. elegans were performed with Bristol wild-type strain (N2). Worms were treated in the first larval stage (L1), which were obtained by a synchronization process. The larvae (2.000 per group) were exposed for half an hour (acute exposure) and after washing with buffered saline $(\mathrm{NaCl} 85 \mathrm{~mm})$. For survival analysis we performed a dose-response curve and plotted sigmoidal dose response curve using nonlinear regression followed by Bonferroni post hoc test. For brood size and Paraquat-induced stress we performed oneway ANOVA and Tukey post hoc. $\mathrm{P}$ values $<0.05$ were considered statistically significant.

Results: The extracts of yerba mate increased survival without altering brood size, in C. elegans at all tested concentrations. There was also an increased resistance to paraquat in these nematodes.

Conclusions: The extracts of yerba mate showed no toxic effects on the nematode $C$. elegans and also exerting a protective activity against the oxidative damage induced by Paraquat, suggesting that the preparation has an antioxidant potential in this model.

\section{P148 \\ Evaluation of Toxic Effects of Iron in Caenorhabiditis Elegans}

\author{
Daiandra Fagundez, Daniela Freitas Câmara, \\ Maria Eduarda Lima, Willian Goulart Salgueiro, \\ Ana Thalita Gonçalves Soares, Daiana Silva Avila \\ Universidade Federal do Pampa
}

Iron is a biologically important metal, being the most abundant metal in the human body, obtained in the diet. This metal has been described as an important element in the pathogenesis of the mechanisms of neurodegeneration. The Caenorhabditis elegans, are tiny worms that live in soil, with a length up to $1.2 \mathrm{~mm}$ in adulthood. Among the main advantages of using this model points to its easy handling and maintenance in laboratory and the fact that they have several homologies with mammals, making it an extremely effective model can be an alternative method of great potential to replace other animal models. The objective of this study was to evaluate the toxic effects of iron in C. elegans. The nematodes used in this study were wild-type N2, strains were maintained on nematode growth medium (NGM) / E. coli OP50 at $20^{\circ} \mathrm{C}$. The worms in the first larval stage ( 2500 per group) obtained by synchronization process were exposed to concentrations of FeSO4 of $0.05 \mathrm{mM}, 0.1 \mathrm{mM}, 0.5 \mathrm{mM} 1 \mathrm{mM}, 1.5$ $\mathrm{mM}$ and $2.0 \mathrm{mM}$ for 30 minutes (acute exposure). After the exposure period the worms were washed with $0.5 \%$ buffer saline and placed on NGM/OP50 new plate. In the survival tests animals grown for 24 hours and then were counted and the average survival were compared to those of untreated animals, for the testing of egg-laying (Brood Size) one animal of each NGM plate and the control group highest concentration were transferred to individual plates, the egg lay was 
monitored daily until the end of the fertile period of four days. After 48 hours was performed to evaluate the behavior of animals. Three experiments were performed in duplicate. The survival curves shown decay of the number of live nematodes with increasing concentrations of iron. In the egg-laying trials there was a delay of about one day in the posture of the animals treated compared to untreated. The behavior assay shows that the treated animals have lower motility (indicated by the number of head movement) and a lower response to touch stimulus. New experiments are being conducted to elucidate the neurotoxicity effects of iron, this study may help to understand the pathogenic processes caused by iron intoxication in humans.

\section{P149 \\ Palmitoleic Acid (Omega-7) Increases ATGL and HSL Gene Expression and Improves Lipolysis in Adipocytes Via PPARA}

\author{
Andressa Bolsoni Lopes, William Tadeu Festuccia, \\ Talita S.M. De Faria, Leonardo Torres Leal, Patrícia Chimin, \\ Rui Curi, Fabio Bessa Lima, Maria Isabel Cardoso Alonso- \\ Vale \\ Federal University of Sao Paulo, Diadema, SP
}

Background: Palmitoleic acid $(16: 1, \omega-7)$, a fatty acid produced in white adipose tissue (WAT) by de novo lipogenesis, was recently shown to modulate insulin sensitivity and triacylglycerol (TAG) metabolism in muscle and liver. However, its effects on WAT metabolism are not fully clarified.

Objectives: To investigate the effects of $\omega^{-7}$ on TAG lipolysis and the lipolitic proteins expression in 3T3-L1 cels and in adipocytes isolated from mice [wild type (WT) and PPAR $\alpha$ knockout (KO)].

Methods: For primary culture studies, epididimal adipocytes were isolated from C57 B6 mice WT and PPAR $\alpha$ KO mice supplemented with $\omega-7(300 \mathrm{mg} / \mathrm{kg} /$ day $)$ or $\mathrm{H}_{2} \mathrm{O}$ by gavage during 10 days. In addition, 3T3-L1 preadipocytes were cultured in growth medium (DMEM) until confluence. Differentiation was induced two days post-confluence by adipogenic cocktail for $48 \mathrm{~h}$. Six days after differentiation, the cells were treated (or not) with $\omega^{-7}(200 \mu \mathrm{M})$ for $24 \mathrm{~h}$. The rate of lipolysis was estimated by Free Glycerol Kit. PPAR- $\alpha$ (GW6741) and PPAR- $\gamma$ (GW9662) antagonists were associated to the treatment in order to access the involvement of PPARs in the lipolytic response. The mRNA of the lipolytic proteins (ATGL, HSL, MGL, CGI-58, G0S2 and perilipin) were estimated by real time PCR.

Results: Treatment with $\omega^{-7}$ stimulated the lipolysis in both, 3T3-L1 cells $(\omega-7: 245.3 \pm 24.1$ vs vehicle: $172.2 \pm 12.1$ and $\omega-7$ : $776.5 \pm 42.5$ vs vehicle: $567.8 \pm 39 \mathrm{nmol} / \mathrm{mg}$ protein, basal and isoproterenol stimulated, respectively) and in primary adipocytes isolated from WT mice supplemented with $\omega-7$ (WT $+\omega-7: 1925 \pm 116$ vs $\mathrm{WT}+\mathrm{H} 2 \mathrm{O}: 1580 \pm 127$ and $\mathrm{WT}+\omega-7: 3825 \pm 54$ vs $\mathrm{WT}+\mathrm{H} 2 \mathrm{O}$ : $2823 \pm 382 \mathrm{nmol} / 106$ cells, basal and isoproterenol stimulated, respectively). In agreement with these data, treatment with $\omega-7$ increased the expression of the lipolytic enzymes HSL and ATGL (around 50\%), but did not influence the expression of MGL, G0S2, CGI58 and perilipin. When PPAR- $\alpha$ antagonist was associated to the 3T3-L1 culture treatments, the effect of the $\omega^{-7}$ on lipolysis was abolished. In the same way, oral supplementation of PPAR $\alpha$ KO mice with the palmitoleic acid resulted in absence of the lipolytic effect in the WT mice, suggesting that PPAR $\alpha$ is an important mediator of the positive effects of $\omega^{-7}$ on lipolysis.

Conclusions: Our data suggest that palmitoleic acid $\left(\omega^{-7}\right)$ increases the lipolytic activity of 3T3-L1 and primary adipocytes. This increament is accompanied by an increase of ATGL and HSL mRNA expression and seems to be mediated by PPAR- $\alpha$ pathway.

Financial support: FAPESP (11/51627-8).

\section{P150 \\ Nutritional Comparison Between Traditional Cookie and Cookie Modified with Green Banana Flour for Celiac Consumer}

\author{
Mariana Galvão B. Cadioli, M. Auxiliadora de Brito Rodas, \\ Maria Lima Garbelotti
}

\section{Instituto Adolfo Lutz}

Celiac disease (CD) is an immune-mediated enteropathy that cause permanent intolerance to gluten in genetically predisposed individuals, and which affects approximately 0.3 to $1.0 \%$ of the population. It is characterized by chronic inflammation on the small intestine, and may develop neoplasm in serious cases. Gluten is a protein fraction present in wheat, rye, oats and other cereals. The intake of individuals and intolerance celiac gluten prevent the adequate absorption of nutrients. A totally gluten-free diet is hard to be followed and may be transgressed voluntarily or involuntarily. The only current treatment for CD is a gluten-free diet, which is not a simple task due to problems adapting to modified products as well as difficulty finding gluten-free products on the market. Furthermore, modified food products lose sensory and technological characteristics, and have increased lipid fractions in order to compensate the modifications. The green banana flour is gluten-free and successfully used by the industry. It is rich in fiber, resistant starch, protein and minerals. The work studied cookie formulations based on wheat flour in comparison to modified with green banana flour, assessing nutritional composition. Traditional cookie formulations were developed based on flour (CFT) and total replacement of this by green banana flour (CFB). Processing and incorporation of ingredients based on data from the literature, whose ratio was calculated on the basis of farinaceous from $100 \% \mathrm{w} / \mathrm{w}$.The physico-chemical (IAL, 2005) encompassed moisture, ash, lipids, protein and dietary fiber, with calculation of carbohydrates and energy value. Statistical analysis employed the Student $t$ test a $5 \%$ error. There was a slight difference $(\mathrm{p}<0.05)$ between the nutritional composition of CFT and CFB. The modified product showed higher carbohydrate content $(77,80 \mathrm{~g} / 100 \mathrm{~g})$ and fiber $(4,45 \mathrm{~g} / 100 \mathrm{~g})$ and lower in protein $(3.74 \mathrm{~g} / 100 \mathrm{~g})$. There was no significant variation in energy value. The higher protein content of the CFT is characteristic of wheat flour, however, the CFB had higher fiber content, a source in the nutrient. The modified product glutenfree food can be seen as a viable alternative for celiac food individuals, acting in the diet for the functional, nutritional and energy, in addition to low-cost access to important poorest people. 


\section{P151 \\ Mechanisms of Antidiabetic Action of Pomegranate (Punica Granatum L.)}

\author{
Mariana Séfora B. Sousa, Amanda C.C.C. Carlos,
} Michel Cardoso de Angelis-Pereira

\section{Sao Paulo University}

Diabetes mellitus (DM) is a metabolic disorder, in which the body either fails to generate insulin (type 1) or the insulin that is formed is not effectively used (type 2), resulting in hyperglycemia. It is associated with lipid metabolism disorder, oxidative stress and inflammation. Various parts of Pomegranate (Punica granatum L.) have been target of current interest as a medicinal agent with antidiabetic action (hypoglycemic, antioxidant and antiinflammatory), probably due to the phenolic compounds. Pomegranate extracts (PE) are rich sources of ellagitannins, gallotannins (punicalin, pedunculagin, granatin B) and anthocyanins (delphinidin, cyanidine and pelargonidin). However, the molecular basis of the observed effects is not fully revealed. Pomegranate peels and flowers were reported to decrease blood glucose, improve insulin levels and number of beta cells in diabetic rats. Studies also suggest that PE leaves improve postprandial hyperglycemia in type 2 diabetes, at least in part, by inhibiting intestinal alpha glycosidase activity. Regarding to in vitro analyzes, seed and hull extracts inhibit aldose reductase enzyme. Furthermore, it has been recently shown that flowers of pomegranate enhance expression of PPAR- $\gamma$ mRNA and restore the reduced expression of glucose transporter (GLUT)-4 mRNA in diabetic rats. Juice, seed and pomegranate peel possess potent antioxidant properties by decreasing nitric oxide and increasing of paraoxonase 1 , superoxide dismutase and glutathione peroxidase expression in diabetic patients. Moreover, administration of PE significantly decreases the concentrations of malondialdehyde, hydroperoxides and inhibits low-density lipoprotein (LDL) oxidation. Recent studies also have documented that PE inhibit inflammation in vivo by inhibition of phosphorylation of ERK1/2, p38 and JNK1/2, as well as activation of IKK- $\alpha$ and transcription factor NF-kB and phosphorylation and degradation of IKB$\alpha$. Activation of mitogen activated protein kinases (MAPK) and $\mathrm{NF}-\mathrm{kB}$ is intimately associated with the increased gene expression of TNF- $\alpha$, IL- $1 \beta$, MCP-1, inducible nitric oxide synthase and cyclooxygenase 2. Moreover, delphinidin inhibits the histone acetyltransferase (HAT) p300/CBP acting as an antiinfammatory by blocking p65 acetylation. Therefore, pomegranate shows therapeutic promise as an agent against the DM. However, further studies are necessary to understand out the exact mechanism by which PE could be exerting its effects.

\section{P152 \\ Molecular Mechanisms of Action of Grape and Red Wine Polyphenols Against Oxidative and Inflammatory Processes in Atherosclerosis}

Mariana Séfora B. Sousa, Amanda C.C.C. Carlos, Michel Cardoso de Angelis-Pereira

Sao Paulo University

Research has shown that initiation and progression of atherosclerosis are associated with the oxidation of LDL-c and secretion of inflammatory cytokines. On the other hand, epidemiological studies have reported association between the consumption of phenolic compounds and reducing of incidence of cardiovascular diseases, which may occur by antiinflammatory and antioxidant effects of polyphenols, for instance resveratrol. Although the mechanisms are not fully understood, studies indicate that phenolic compounds possibly complex with metals (such as iron and copper), or else they increase the antioxidant activity of plasma and also preserve the activity of enzyme paraoxonase. Moreover, the polyphenols neutralize free radicals and activate transcription factors, for example nuclear factorerythroid 2-related factor 2 (Nrf2), which increases the expression of genes that encode important proteins in antioxidant defense (such as superoxide dismutase and glutathione peroxidase). Grape and red wine polyphenols modulate the activity of activator protein 1 (AP-1) by inhibiting protein kinase $\mathrm{C}$ (PKC) activation and mitogen activated protein kinases (MAPK) and they can also inhibit the activity of cyclooxygenase and lipooxygenase enzymes. Additionally, resveratrol inhibits I $\kappa \mathrm{B} \alpha$ phosphorylation, $\mathrm{p} 65$ phosphorylation and $\mathrm{NF}-\kappa \mathrm{B}$ p65 subunit translocation from cytosol to nucleus. The combined inhibition of NF-kB and AP-1 results in the block of the synthesis of cytokines, especially TNF- $\alpha$, chemokines and adhesion molecules. Regarding in vitro analyses, the down regulation of gene and protein expression of VCAM- 1 and interleukin- 6 and -8 by extracts of grape was associated with increased expression of the microRNA-126. Another proposed mechanism is the action of polyphenols over deacetylases. Resveratrol activates histone deacetylase type 2 (HDAC2) and sirtuin 1 (SIRT-1), proteins able to remove acetyl groups from histones, and thereby preventing binding of transcription factors, such as NF-kB, to the DNA. SIRT-1 activation by resveratrol is also involved in the induction of transcription factor Krüppel-like factor 2 (KLF-2) in human endothelial cells. Although the actions of KLF-2 are not fully understood, its activation has been implicated in the modulation of angiogenesis by antithrombotic, antiadhesive and antiinflammatory properties. These actions together reduce oxidation of LDL-c and inflammatory process, attenuating the atherogenic process. 
P153

\section{Domain Swap Chymeras Between Thyroid Hormone Receptor Alpha and Beta Isoforms: Strategy to Understand Structure-Function Differences}

Marie Togashi, Aleksandra Cvoro, Rilva G.P. Soares, Geilliany De O. Campos, Douglas Sieglaff, Paul Webb

Universidade de Brasilia

The understanding of structure-function characteristics of different thyroid hormone receptor (TR) isoforms is regarded as an important tool to aid the design of selective thyromimetics. There is a high homology between the two isoforms and the few differences observed in primary sequences account for well-known distinguished responses. Preliminary results using TR alpha and TR beta stable cell lines have shown that TR subtypes activate highly similar gene sets but with wide variations in the magnitude, kinetics/potency of response and response to selective ligands. We performed domain swaps between the two TR isoforms (chymeras) and deletions of the $\mathrm{A} / \mathrm{B}$ domain in expression vectors to address which regions of the receptor are responsible for differences in TR action on ideal consensus thyroid hormone positively regulated response elements (REs), DR4 and IP6, and a negatively regulated one, TSH alpha. Preliminary results obtained by gene reporter assays in transiently transfected HeLa cells suggest that TR responses on DR4 seem not to be influenced by A/B domain deletion, but that higher levels of TR beta activation at IP6 elements and greater hormone dependency of repression at the TSH alpha element are functions of $\mathrm{A} / \mathrm{B}$ domains. We are performing a detailed characterization of the TR alpha and TR beta chimeras from cofactor exchange and affinity, transcriptional activation to oligomeric formation on REs to understand the role of each domain in different REs and use them to understand TR action in natural promoters.

Financial support: Fundação de apoio à pesquisa do df (FAPDF) (193.000.527/2009).

\section{P154}

\section{Association of PPARG, LEPR and APM1 Gene Polymorphisms with Dietary Intake and Blood Lipids Levels in Children}

\author{
Marília Remuzzi Zandoná, Raquel Olmedo Rodrigues, \\ Silvana Almeida, Paula Dal Bó Campagnolo, \\ Márcia Regina Vitolo, Vanessa Suñe Mattevi \\ UFCSPA
}

Background: The prevalence of childhood obesity is increasing worldwide and confers risks for early type 2 diabetes, cardiovascular disease and metabolic syndrome. Increased energy intake is a significant risk factor of overweight and obesity. Peroxisome proliferatoractivated receptor (PPARG) influence energy intake, satiety responsiveness, intake of palatable energy-dense food and body mass index determination. Furthermore, leptin and its receptor, LEPR, are essential in the regulation of food intake and energy expenditure.
Adiponectin (APM1), the most abundant adipocyte-derived protein, exhibits anti atherosclerosis, anti diabetic as insulin sensitizer, and anti inflammatory properties and hypoadiponectinemia has been observed in association with obesity.

Objectives: We analyzed these polymorphisms, rs1801282 (PPARG gene), rs1137101 (LEPR gene) and rs17300539 (APM1 gene), to investigate association these genetic variations with food intake, anthropometric and metabolic parameters in 305 Brazilian children at eight years old, who were enrolled in a cohort study since birth.

Methods: Polymorphisms were analyzed using polymerase chain reaction-based procedures and their association with dietary, anthropometric and metabolic data was evaluated by t-tests, analysis of variance, Mann-Whitney test, Kruskal Wallis test and general linear models.

Results: The PPARG rs1801282 and APM1 rs17300539 polymorphisms were associated with palatable foods intake. Children carrying the PPARG Ala-allele presented higher intake of lipid-dense foods $(P=0.010)$, sugar-dense foods $(P=0.020)$, total energy intake $(\mathrm{P}=0.037)$, total amount of fat intake $(\mathrm{P}=0.039)$ and marginal differences in total carbohydrates intake $(\mathrm{P}=0.056)$ when compared with the Pro/Pro genotype. For APM1 rs17300539, the amount of total carbohydrates intake were higher in $\mathrm{G} / \mathrm{G}$ individuals $(\mathrm{P}=0.019)$ and a trend of association was observed in total energy intake $(\mathrm{P}=0.059)$. The LEPR rs1137101 was associated with plasma lipids. Carriers of Gln/Gln genotype presented higher HDL cholesterol levels than Argallele carriers $(\mathrm{P}=0.044)$, and higher LDL cholesterol levels and lower triglycerides levels in relation to Arg/Arg homozygotes ( $\mathrm{P}=$ 0.021 and 0.024 , respectively).

Conclusions: This study provides indications that genetic variants in PPARG and APM1 genes might be involved in determination of palatable foods intake, and LEPR gene polymorphisms might influence metabolic profile in children.

\section{P155 \\ Gut Microbiota, Dairy Foods and Obesity: A New Way for Applying Nutrigenomics to Chronic Diseases}

Marina Andrade Batista, Larissa Lovatto Amorin, Natália Cruz Campos, Marialice Pinto Silvestre

Universidade Federal de Minas Gerais

This review is about gut microbiota, nutrigenomics and obesity. Obesity is considered the major global public health problem, originated from the actual life style, which contributes to the development of many other diseases ${ }^{1}$. According to a recent study, gut microbiota, food and human host interact and play a response in nutrigenomics allowing the optimization of a personalized nutrition ${ }^{2}$. A review paper showed that most studies with animals and humans clearly specify that gut microbiota is different between lean and obese individuals ${ }^{1}$. This is important since the gut microbiota regulates glucose uptake from the small intestine, energy metabolism, de novo lipogenesis in the liver, fat storage, serum triglycerides, enterohepatic circulation of bile acids ${ }^{1}$ and blood pressure ${ }^{3}$. Obesity is associated with a lowgrade inflammation, which might be molecularly modulate by using 
probiotics $^{1,3}$. Thus, the intestine is promising as a target organ to design foods with novel health-promoting function, such as probiotics, prebiotics and short-chain fatty acids ${ }^{3}$, which regulate satiety and food intake, through control of gut hormone expression ${ }^{1}$. Peptides derived from milk and dairy products, for example, appear to act in gut microbiota and may have beneficial properties in blood pressure, oxidative stress, homeostasis, appetite, and lipid metabolism ${ }^{2}$. Humans are characterized by genetic pre-disposition and inter-individual variability in terms of response to nutritional interventions, epigenetic, metabolic programming at certain life stages, and acute genomic expression as a holistic response to diet, monitored at gene transcription, protein and metabolite level ${ }^{2}$. In fact, more studies are needed to appreciate details concerning the role of bioactive peptides derived from milk in gut microbiota and obesity, being an innovator alternative to several chronic diseases.

References: 1. Conterno L, Fava F, Viola R, Tuohy KM. Obesity and the gut microbiota: does up-regulating colonic fermentation protect against obesity and metabolic disease? Genes Nutr 2011;6:241260 .

2. Kussmann M, Van Bladeren PJ. The extended nutrigenomics understanding the interplay between the genomes of food, gut microbes, and human host. Front. Genet 2011;2:1-13.

3. Shimizu M, Hachimura S. Gut as a target for functional food. Trends Food Sci Tech 2011;22:646-650.

Financial support: CNPQ and FAPEMIG.

\section{P156 \\ Recent News About Obesity, Nutrigenomics and Functional Compounds}

\section{Marina Andrade Batista, Larissa Lovatto Amorin, Natália Cruz Campos, Marialice Pinto Silvestre \\ Universidade Federal de Minas Gerais}

This review is about novelties concerning the obesity, nutrigenomics and functional compounds. Actually, we know that obesity is a global health problem, which is associated with several risk factors, chronic diseases, and has environmental and genetic causes ${ }^{1}$. Inflammation, oxidative stress and lower blood flow in the large adipose tissue masses are also related to this pathologic condition ${ }^{2}$. The literature has shown some alternatives to treat this impacting disease, such as the action of functional compounds in genes expression. Proanthocyanidins attenuate mitochondrial dysfunction caused by diet-induced obesity in rats either by improving sirtuin 1, nuclear respiratory factor 1 , isocitrate dehydrogenase $3 \gamma$ and $\operatorname{COX} 5 \alpha$ or by decreasing the levels of mitochondrial respiration both with pyruvate and canitine-palmitoyl-CoA ${ }^{1}$. PUFA supplementation reduces LDL-C and TAG levels, while MUFA increases adiponectin expression by PPAR- $\gamma$-activating ligands in subjects at increased cardiometabolic risk ${ }^{3}$. The adiponectin promotes insulin sensitivity by activating AMP kinase and PPAR- $\alpha$ in liver and skeletal muscle ${ }^{3}$. A study with obese individuals observed that polymorphism of the interferongamma-inducible protein 30 gene in the visceral adipose tissue may be associated with cardiovascular disease risk factors, such as hyperglycemia and diabetes ${ }^{4}$. The search for functional compounds and adequate nutrition is a strategy that might modulate metabolism path- ways and immunity at the molecular level in obesity, which promotes health and allows a personalized nutrition to prevent chronic diseases.

References: 1. Pajuelo D, Quesada H, Díaz S et al. Chronic dietary supplementation of proanthocyanidins corrects the mitochondrial dysfunction of brown adipose tissue caused by diet-induced obesity in Wistar rats. Br J Nutr 2012;107:170-178.

2. Alemany M. Regulation of adipose tissue energy availability through blood flow control in the metabolic syndrome. Free Radical Biol Med 2012;52:2108-2119.

3. AlSaleh A, Sanders TAB, O'Dell SD. Effect of interaction between PPARG, PPARA and ADIPOQ gene variants and dietary fatty acids on plasma lipid profile and adiponectin concentration in a large intervention study. Proc Nutr Soc 2012;71:141-153.

4. Turcot V, Bouchard L, Faucher G et al. A polymorphism of the interferon-gamma-inducible protein 30 gene is associated with hyperglycemia in severely obese individuals. Hum Genet 2012;131:57-66.

Financial support: CNPQ and FAPEMIG.

\section{P157 \\ Effect of High Refined Carbohydrate- Containing Diet on Metabolic and Inflammatory Parameters in Adipose Tissue of Mice}

Marina Chaves Oliveira, Zélia Menezes-Garcia, Frederico Marianetti Soriani, Vanessa Pinho, Danielle Glória Souza, Mauro Martins Teixeira, Adaliene Versiani M. Ferreira

Universidade Federal de Minas Gerais

Background: Diet composition affects the expression of some genes and, consequently, the phenotypic expression. However, the effect of genes expression caused by high refined carbohydrate-containing diet (HC) on inflammatory parameters and metabolic disarrangement of adipose tissue are poorly understood.

Objectives: To evaluate the timing and progression of metabolic and inflammatory dysfunction induced by $\mathrm{HC}$ diet in mice.

Methods: BALB/c mice were fed chow or HC diet for 1 and 3 days, 2, 8 and 12 weeks. Food intake, body weight gain, adiposity index and adipose morphometry were performed. Adipose gene expression of ACC, LPL, PPAR $\gamma$, SREBP-1 and ChREBP were analyzed by real-time PCR. Serum levels of total cholesterol, triglycerides, glucose, adiponectin, resistin and leptin were measured. To evaluate the systemic and adipose tissue inflammation the total and differential count of blood leukocytes were analyzed, the concentration of TNF- $\alpha$, IL-6, IL-10, TGF- $\beta 1$, CCL2, CXCL1 and intravital microscopy in adipose tissue performed.

Results: On average, body weight gain and food intake did not change in mice given chow or $\mathrm{HC}$ diet at all times evaluated. However, mice given $\mathrm{HC}$ diet exhibited a rapid (after 1 day) increase in adipose tissue mass and adipocyte hypertrophy. The expression of ACC, LPL, PPARy, SREPB-1 and ChREBP increased at 1 day after $\mathrm{HC}$ diet and return to basal levels in the other times evaluated. The mice presented hyperlipemia, hyperglycemia and altered circulating levels of leucocytes, adiponectin, resistin and leptin. There was leucocyte rolling and adhesion on adipose tissue microvessels at 3 days 
and until 8 weeks of $\mathrm{HC}$ diet. Concentrations of TNF- $\alpha$ and IL- 6 increased significantly in adipose tissue of mice fed $\mathrm{HC}$ diet throughout the experimental period. However, IL-10 and TGF- $\beta 1$ increased continuously till the second week of the start of $\mathrm{HC}$ diet and tended to fall thereafter to baseline levels at week 12. Concentrations of CCL2 increased from day 1 after the start of $\mathrm{HC}$ diet and peaked at week 1. Thereafter levels fell very significantly but were still above baseline levels in mice given $\mathrm{HC}$ diet at all time points. The adipose CXCL1 levels were transiently increased in mice fed $\mathrm{HC}$ diet throughout the experimental period.

Conclusions: The acute inflammatory response in adipose tissue occurs very early and is sustained suggesting that adipose tissue inflammation is a homeostatic mechanism to regulate nutrient overload and adipose expansion.

Financial support: CAPES, CNPQ and FAPEMIG.

\section{P158 \\ Effect of Isocaloric Intake of a High-Fat Diet on Insulin Signaling Pathway in White Adipose Tissue in Wistar Rats}

Marina Maintingue Norde, Mônica Yamada,

Francisco Leonardo Torres-Leal,

Maria Isabel Cardoso Alonso-Vale, Mirian Helena Fonseca-

Alaniz, Tatiane Mieko Fujii, Patrícia Silva Jacob,

Maria Carolina Borges, Julio Tirapegui, Primavera Borelli,

Ricardo Ambrósio Fock, Marcelo Macedo Rogero

Faculdade de Saúde Pública da USP

Background: Obesity, type 2 diabetes, and cardiovascular disease share a metabolic milieu characterized by insulin resistance and chronic subacute inflammation. The amount and the type of dietary fatty acid can modulate inflammatory signaling pathways in white adipose tissue (WAT). High-fat diet (HFD) impairs insulin-stimulated glucose transport and uptake rates, in part, due to increased insulin receptor substrate-1 (IRS-1) serine phosphorylation.

Objectives: The aim of this study was to investigate the effect of isocaloric intake from a HFD on insulin signaling pathway in white adipose tissue in rats.

Material and Methods: Male Wistar rats, aged two-months old, were randomly assigned to one of two groups: control (CON) group ( $9 \%$ of calories from fat) and the HFD group ( $60 \%$ of calories from fat) $(n=6 /$ group$)$. Food intake was measured three times a week. After 12 weeks, the oral glucose tolerance test (oGTT) and the intraperitoneal insulin tolerance test (ipITT) were performed. Also, homeostasis model assessment (HOMA) was used as an indicator of peripheral insulin resistance, using blood glucose ( $\mathrm{mmol} / \mathrm{L})$ multiplied by insulin $(\mu \mathrm{IU} / \mathrm{mL})$ fasting as parameters, and divided by 22.5 . Periepididymal adipose tissue homogenate was used to measure the activation of insulin signaling pathway by quantifying the expressions of IRS-1 and AKT, in its total and phosphorylated forms, by the Western Blotting technique. Prior to analysis, results were checked for distribution normality and homoscedasticity, and means from the control and HFD groups were compared by unpaired Student's t-test.

Results: Food intake (g) in the HFD group was lower in comparison to the CON group, however, there were no differences in energy intake between the groups $(p<0.05)$. Fasting glucose was significantly higher in rats fed a HFD $(p<0.05)$, but there was no statistical difference in plasma insulin levels or HOMA compared to the CON group. Furthermore, the consumption of a HFD resulted in glucose intolerance and impaired insulin sensitivity when animals were submited to the oGTT and the ipITT $(\mathrm{p}<0.05)$. However, no statistical difference was found related to the phosphorylation of IRS-1 (Ser307) and AKT (Ser473) proteins between the groups.

Conclusions: Isocaloric intake of HFD induced insulin resistance and glucose intolerance in rats, however, this diet was not associated with altered insulin signaling pathway in white adipose tissue.

Financial support: FAPESP and CNPQ (2009/54395-0 AND 470418/2009-5).

\section{P159 \\ Effects of Increased Antioxidant Capacity in Cells of Skeletal Muscle Insulin Resistance}

Marina Rodrigues Barbosa, Luciane C. Alberici, Isis Kettelhut, Igor Sampaio, Leonardo dos Reis Silveira

Universidade de São Paulo

Reactive oxygen species are involved in various physiological processes in skeletal muscle. However, the high availability of fatty acids induces insulin resistance. The pathogenesis of insulin resistance involves disorders of lipid metabolism. Although the mechanism is still unclear, high levels of ROS are involved in the condition of insulin resistance. Therefore, we examined whether the reduction of mitochondrial capacity and the insulin response imposed by the high availability of fatty acids are associated with an increase of intracellular hydrogen peroxide (H2O2). The skeletal muscle cells were isolated from quadriceps muscle from Wistar rats and cultured in DMEM medium. The cells were transfected with pcDNA3 plasmid in medium containing Lipofectamine (1:4). After differentiation, the cells were exposed to the palmitic acid $(700 \mu \mathrm{m})$ during $72 \mathrm{~h}$. After this period, the cells were collected and the CAT content and mRNA were determined as a control experiment. In addition, the citrate synthase activity (CS) and the mRNA level of PGC1 alfa were examined as mitochondrial biogenesis factors. Protein content of insulin receptor (IR) and Akt defined by the Western Blotting. The extracellular ROS production were determined by Amplex fluorescent probe. Whereas the oxygen consumption was measured at presence of glucose $(5.6 \mathrm{mM})$ with or without insulin $(0.1 \mathrm{U})$ using a Clark-type electrode. In addition, glucose uptake and fatty acids oxidation determinated by radioactive method. The catalase transfection was successfully transfected as indicated by catalase mRNA and content $(p<0.05)$. The palmitic acid treatment markedly reduced the citrate synthase activity as well as the mRNA levels of PGC1 $\alpha(\mathrm{p}<0.05)$. This effect was accompanied by an elevated production of $\mathrm{H} 2 \mathrm{O} 2$ but reduction of oxygen consumption, glucose uptake and fatty acid oxidation $(\mathrm{p}<0.05)$. In addition, the levels of PGC1 $\alpha$, PPAR $\beta$, phosphorylated Akt and citrate lyase were markedly reduced. However, the catalase transfection was observed to attenuate this effect $(p<$ 0.05 ). Our results showed that elevated fatty acid availability reduces mitochondrial oxygen consumption favoring ROS production and insulin resistance. The improvement in the oxidative capacity of the 
cells by CAT overexpression improved mitochondrial biogenesis, oxygen consumption, as well as glucose uptake, suggesting that the control of intracellular ROS is an important therapeutic target in protecting against type 2 diabetes in muscle cells.

Financial support: CAPES, CNPQ E FAPESP.

\section{P160 \\ Protein Restriction in Early Life Impairs the Increase in First-Phase Insulin Secretion During Pregnancy with No Change in MRNA of Proteins Related to Exocytosis}

Bianca Karine Marin, Sílvia Regina de Lima Reis, Albina de Fátima Silva Ramalho, Simone Ferreira Lemes, Leonardo Marin, Emerielle Cristine Vanzela, Everardo Magalhães Carneiro, Márcia Queiroz Latorraca, Vanessa Cristina Arantes, Marise A. De Barros Reis

Universidade Federal de Mato Grosso-UFMT

Background: During normal pregnancy gradual maternal insulin resistance, which is accompanied by increased insulin secretion, develops to provide fuel to the developing fetus. It is known that malnutrition in intrauterine life may reduce insulin secretion. Nutritional deficiencies during prenatal or postnatal life lead to metabolic programming that may increase the chances of developing diabetes mellitus and metabolic syndrome in adult life. Alterations such as reduced islet size, $\beta$-cell proliferation and decreased capacity of glucose to increase $\mathrm{Ca}^{2+}$ uptake and/or to reduce its efflux have been associated with the lower insulin secretion observed as result of malnutrition.

Objectives: Investigate the kinetics of glucose-induced insulin secretion and its relationship with intracellular calcium concentration $([\mathrm{Ca} 2+] \mathrm{i})$ and gene expression of proteins from exocytotic machinery in pancreatic islets from recovered pregnant and long-term protein-restricted pregnant rats.

Methods: Female rats were separated in control-fed pregnant (CP) and non-pregnant (CNP) or protein-restricted pregnant (LPP) and non-pregnant (LPNP) rats, from fetal to adult life, and in proteinrestricted rats that were recovered after weaning (RP and RNP). Islets were isolated by collagenase method to evaluation of insulin secretion, $[\mathrm{Ca} 2+] \mathrm{i}$ by fluorescence, protein expression by Western-blot and quantitative Real Time-PCR.

Results: In RP and CP rats, glucose-induced biphasic insulin secretion increased with a rapid 1st-phase release after exposure to high level of glucose, followed by decrease, and then an increase during the second phase. LPP islets exhibited a monophasic pattern with a small peak of insulin release, followed by maintenance of the constant values. Insulin secretion during the 1st-phase was similar in RP and RNP as well as in LPP and LPNP groups but was higher in CP than in CNP group. The $[\mathrm{Ca} 2+]$ i from LPP and LPNP islets was lower than in control groups and both were lower in relation to RP and RNP groups. SNAP-25 was increased in islets from pregnant rats independently of their nutritional status and syntaxin-1 level was reduced in RP in relation to RNP rats. VAMP2 content was similar among the groups. No change was observed in mRNA of these proteins.
Conclusions: Protein restriction during intrauterine life and lactation increased $[\mathrm{Ca} 2+] \mathrm{i}$, but impaired the classical first phase of insulin release during pregnancy, due at least in part to reduced Sintaxin-1 protein expression.

Financial support: CNPQ, CAPES (CNPQ-620106/2008-5, 479362/2011-4, PROCAD 022/2007).

\section{P161 \\ Proteomic Approach to Evaluate the Role of Cocoa Consumption in the Inflammatory Pathway}

Marta Roccio, Giorgio Grosa, Silvio Aprile, Stefania Moia, Gillian Elisabeth Walker, Flavia Prodam, Simonetta Bellone, Marco Arlorio, Gianni Bona

Department of Health Sciences, UNIPMN

Background: Diet has been demonstrated to play a crucial role not only for the reduction of excessive energy intake but also for the normalization of tissue hyper-inflammation, that is characteristic of obesity. Dietary components such as polyphenols present in cocoa (Theobroma cacao L.) have important health properties including antioxidant properties, decreased platelet activation and function, modulation of immune function and inflammation. Monocytes are key to this inflammatory status and offer an important key in investigating the role of dietary components and their metabolites.

Objectives: The main target of this research was to study the key molecular mechanisms through which the bioactive components of cocoa may modulate the inflammatory state in healthy subjects.

Methods and Results: At present, there is no specific report on the efficacy of bioavailable flavanols on inflammation in humans, despite promising data in animal models. For this reason a liquid chromatography-mass spectrometry (LC-MS) analysis was performed to evaluate the bioavailability of epicatechin, in particular the glucuronide metabolite, in the plasma of five healthy volunteers (young men aged 22-27 years) treated with acute and chronic levels of cocoa. Cocoa was administered in the form of chocolate bars, derived from two batches of chocolate with different percentages of cocoa (40\% and $80 \%)$.

At the same time, peripheral mononuclear cells were isolated from the blood of the same subject, before and after administration. Cells were lysed and analyzed by 2D-electrophoresis with IPG strips 5-8 $\mathrm{pH}$, with the respective proteomic profiles compared using PDQuest statistical software. Overall, the analysis showed an average total of 705 spots $(n=5)$ present within the monocytes isolated. Following a deeper analysis, our attention was drawn to a cluster of spots localized between 30 to $60 \mathrm{KDa}$, where we observed differences in protein expression after three hours of administration of cocoa, when compared with the basal profile.

Conclusions: The data obtained from the bioavailability experiments and proteomic analyses represent the initial phase of an ongoing study that will contribute to understanding the role of diet on inflammatory mechanisms linked to cocoa consumption. In this regard confirmational experiments will be performed to investigate the hypothetical role of these proteins with inflammatory pathways. 


\section{P162}

\section{Functional Foods Based on Whey Proteins with Bioactive Properties Play a Key Role in Helping to Combat Obesity}

\author{
Mayara Franzoi Moreno, Gabriel Inacio de Moraes Honorat \\ o de Souza, Ana Claudia Losinskas Hachul, \\ Marcos Hiromu Okuda, Bruno Santos, Elisa Esposito, \\ Lila Missae Oyama \\ UNIFESP
}

Introduction: Obesity may be partly responsible for increased oxidative stress and consequent alteration of the expression of proinflammatory cytokines in different tissues, which can trigger diseases related to metabolic syndrome. Functional foods with bioactive properties could be an important role in helping to combat obesity, decreasing risks for diseases and improve the physiological positively modulating inflammatory pathways. The aim of this study was to investigate the effects the supplementation of protein coacervate milk whey on the probable mechanisms involved in proinflammatory processes in mice with obesity induced by high fat diet.

Methods: After 12 weeks of treatment ( 8 weeks with diets control (C) or hyperlipidic (HF) +4 weeks of intervention with coacervate (36 mg protein/ $\mathrm{kg}$ body weight/day) or water administered daily through gavage, under controlled light-dark cycle and $22-25^{\circ} \mathrm{C}$. The animals were sacrificed by decapitation and adipose tissues depots and liver were removed. The serum lipid profile was determined using a commercial kit from Labtest. The tissue concentrations of tumor necrosis factor- $\alpha$ (TNF- $\alpha$ ), interleukin-6 (IL-6), interleukin-10 (IL-10) were determined by ELISA using commercial kits and are expressed as total protein. Data analysis was performed using the software Statistica 6.0. The mean and standard deviation (MSD) were calculated for all variables. We used mann-whitney test. The significance level used for all analyzes will be less than $5 \%, \mathrm{p}<0.05$.

Results: High fat diet increased the serum concentration of triacylglycerol: C coacervate 144.23 \pm 16.2 , C $153.2 \pm 8.4$, HF Coacervate $159.1 \pm 9.6$, HF $185.8 \pm 19.3$. Supplementation with coacervate promoted the normalization of this parameter, suggesting that intake of this complex may in part improve the lipid metabolism disorders triggered by eating high-fat diet. In the liver, the concentration of TNF- $\alpha$ $(p=0.004)$ and IL-10 $(p=0.001)$ had increased in the HF group compared to animals treated with HF coacervate. The increased concentration of IL-10 in liver of animals treated high fat diets may suggest an attempt to decrease the body's inflammatory process triggered by high-fat diet, increasing the production of an antiinflammatory cytokine.

Conclusions: The high fat diet increases serum concentrations of triacylglycerol and liver concentrations of TNF and in general the association with the coacervate improves the proinflammatory profile.

Financial support: CAPES/FAPESP (2009/53801-5 E 2011/ 04310-9).

\section{P163}

\section{Anti-Inflammatory Effects of Polyphenol- Rich Araçá (Psidium Cattleianum Sabine) Extract on Macrophages $\mathbf{J 7 7 4}$}

\author{
Mayra Domiciano, Ingrid Candido Gorofolo, \\ Franco Maria Lajolo, Maria Inés Genovese, Silvana Bordin, \\ Rui Curi, Camilo Lellis-Santos \\ University of São Paulo
}

Several researches have presented the benefits of phenolic compounds rich diets due to anti-inflammatory and antioxidant effects of these molecules, which might neutralize free radicals, preventing or ameliorating the damages of metabolic diseases, such as obesity, diabetes and insulin resistance syndrome (SRI). Diets containing fruits from North hemisphere (berries fruits) or Mediterranean foods (olive oil and wine) have been extensively studied. However, putative functional foods or nutraceuticals from Brazilian flora is poorly investigated. Araçá or strawberry guava (Psidium cattleianum Sabine) is a native Brazilian fruit that has not been thoroughly studied or commercially exploited, but it has significant amounts of polyphenols. Attempting to demonstrate an anti-inflammatory effect of the polyphenol rich Araçá extract (PAE), macrophages J774 were treated with doses of 100,500 or $1000 \mu \mathrm{g} / \mathrm{mL}$ of PAE in the presence or absence of $1 \mu \mathrm{g} / \mathrm{mL}$ lipopolysaccharide (LPS) for 24 hours. Cellular viability was not reduced by any dosage, analyzed through flow cytometry. The phenolic extract at $100 \mu \mathrm{g} / \mathrm{mL}$ significantly reduced nitric oxide (NO) produced by LPS-stimulated macrophages, measured by Griess method. This result correlates with the decreased expression of inducible nitric oxide synthase (iNOS), and phosphorylation of p38 Mitogen-activated Protein Kinase, which are markers of activation and oxidative stress in macrophages, respectively. After high performance liquid chromatography (HPLC) identification of the phenolic compounds from Araça extract, LPS-stimulated macrophages were treated with catechin $(264 \mathrm{nM})$, rutin $(16.7 \mathrm{nM})$ and ellagic acid $(43.35 \mathrm{nM})$ or polyphenol mixture at dose equivalent to $100 \mu \mathrm{g} / \mathrm{mL}$ treatment. Only catechin was able to decrease NO production, iNOS e p38. The polyphenol rutin increased proinflammatory actions induced by LPS. The polyphenol mixture was able to reproduce the effects of the extract in all measured parameters. This is the first description of the catechin presence in this fruit. Our data reinforce the benefits that the consumption of catechin-containing foods or nutraceuticals could promote on inflammation status. Therefore, polyphenol-rich Araçá extract should be considered as a putative nutraceutical to prevent and treat metabolic diseases such as diabetes and obesity. 


\section{P164 \\ Topical Antimicrobial Activity of Oleic and Linoleic Acids in Wounds}

\author{
Maysa Braga, Gilson Masahiro Murata, Rui Curi, \\ Andrea Maria Spessoto, Renata Gorjão, Elaine Hatanaka
}

Universidade Cruzeiro do Sul

Background: The benefits of topically treat wounds using fatty acids include the low cost of the treatment and the fact that the oily damp dressings prevent tissue dehydration and reduce trauma when dressings are changed. Previous studies have demonstrated the immunomodulatory nature of fatty acids in signaling tissue repair. Recently, our interest has been focused on the role of fatty acids as anti-microbial agents to eliminate microorganisms that appear at wound site.

Purpose: The aim of this study was to determine whether oleic and linoleic acids offer a natural barrier against microorganisms in the wound healing, based on in vivo and in vitro assays.

Methods: In vivo: In rats with wounds and treated with oleic and linoleic acids, the predominant bacterial species were isolated, selected, and identified. In vitro: Bacteria growth (S. aureus, E. coli, and S. epidermidis) was evaluated by using oleic and linoleic acids $(250-4000 \mathrm{uM})$ in TSB medium. Additionally, oleic and linoleic acids $(250-4000 \mathrm{uM})$ were tested in an agar-based disc diffusion assay and the inhibition halos were measured.

Results: At 250uM and higher concentrations, oleic and linoleic acids inhibited the growth of S. aureus and S. epidermidis. The main antibacterial effects of both fatty acids occurred in the lag phase.

Conclusions: Oleic and linoleic acids can be considered as antibacterial agents for the topical treatment of wounds. This study was conducted with the approval of the Ethics Committee of UNICSUL $(165 / 102011)$ and was financially supported by FAPESP (2008/50310-5 and 2011/19589-9) and CNPq (475841/2011-5 and 306041/2011).

Financial support: FAPESP (2011/19589-9).

\section{P165 \\ Nutritional Genomics: Concepts and Applications}

\section{Meire Oliveira Barbosa, Maria Anete Santana Valente \\ Universidade Federal de Lavras}

The nomenclature for the field of nutritional genomics is still evolving, and it is common to see "nutrigenomics" used as a shorthand version of "nutritional genomics". There are two major subcategories of nutritional genomics: nutrigenetics and nutrigenomics. Nutrigenetics is concerned with the effect of gene variations on the organism's functional ability, specifically its ability to digest, absorb, and use food to sustain life. Depending on the specific gene variant, the person's likelihood of developing a disorder may be increased or reduced. These genes are the primary focus of nutritional genomics, because they are common within the global population, they affect dietary recommendations about the types and amounts of food that best fit a person, and practical interventions are possible. For exam- ple, the $677 \mathrm{C}>\mathrm{T}$ variation in the gene for 5,10-methylenetetrahydrofolate reductase (MTHFR) results in an altered enzyme that, in the absence of adequate folate, increases the risk for colon cancer, fetal neural tube defects, and possibly cardiovascular disease. People with this variation must be particularly diligent in maintaining adequate folate intake and, quite possibly, may need more than the standard recommended amount. Nutrigenomics, in contrast, is concerned with how bioactive components within food affect gene expression and function, reflecting the complex communication that occurs between the environment and an organism's command center. However, keeping the concepts separate can be helpful when sorting out the underlying mechanisms involved. For example, transcription of a number of genes involved in lipid metabolism and oxidation requires the coordinated binding of two transcription factors: the retinoic acid receptor (RXR) and the peroxisome proliferator activator receptor gamma (PPARgamma). These two proteins must form a heterodimer in order to bind to the response element in the regulatory region of these genes. To form the heterodimer, each protein must bind its respective ligand. For RXR, the ligand is a vitamin A derivative, for PPAR gamma, the ligand is a polyunsaturated fatty acid, such as an omega-3 fat. Thus, food components are integral in communicating the state of the environment to the command center and effecting the appropriate response. Such an understanding of the underlying mechanisms, coupled to information about a person's genotype, forms the basis for developing targeted nutritional interventions.

Financial support: FAPEMIG.

\section{P166 \\ Proteomics as a Tool for Functional Genomics: Applications in Nutrition}

Meire Oliveira Barbosa, Maria Anete Santana Valente, Patrí cia Aparecida Fontes Vieira

Universidade Federal de Lavras

Nutrigenomics seeks to understand the influence of nutrients or bioactive food compounds in the nutrient-gene interaction in response to diet. Proteomics is defined as a set of all proteins expressed in a tissue or cell or biological system at some moment cell. The proteome analysis consists in four essential steps: preparation of the sample, separation, identification and determination of the amino acid sequence. Obtaining and preparing of samples represent a critical step in proteomic analysis. The methods of separation of proteins are one electrophoresis or two-dimensional (2-DE) and liquid chromatography (LC). In 2-DE, proteins are separated according to their isoelectric point and molecular weight. To make visible the protein spots, gels are stained, and after this procedure are scanned and the images are analyzed by specific software to identify the differential spots. Parallel the LC has been used to reduce the complexity of samples, separating protein groups with similar characteristics and sample enrichment. The principal method of identifying proteins is used to mass spectrometry. The technique consists of ionization of the protein/peptide and evaluating the mass/charge $(\mathrm{m} / \mathrm{z})$ ions. Two methods used for ionization proteins are: MALDI and ESI. After ionization the ions are separated by analyzers like TOF, quadrupole or ion trap. The end result is a graph of $\mathrm{m} / \mathrm{z}$ versus intensity are compared with infor- 
mation available in databases such as MASCOT and SEQUEST, which enables identification of the protein of interest by peptide mass fingerprinting. The peptides obtained in the spectrum may undergo fragmentation spectrum and the graph MS/MS can provide the resulting amino acid sequence of proteins by de novo sequencing or by peptide fragment fingerprinting. In nutrition, proteomics study aimed at identifying cellular responses to dietary constituents, as well as their molecular targets and how they influence the metabolism and also applied in the qualitative analysis of food and the study of protein-protein interaction in raw foods and/or processed, and the interactions between proteins and other dietary components. It is noteworthy that a single proteomic analysis of a biological material is not able to reflect its entirety, given the variability between individuals and materials and technologies inherent limitations, however the accumulation of data is extremely important to help in customizing diet and diagnosis of disease in the future.

\section{P167 \\ Does Physical Activity Behavior Modulate the Impact of Rs9939609 SNP of FTO on Adiposity-Induced Disturbances?}

\author{
Milena Monfort-Pires, Maira L.R. Curti, \\ Luciana Dias Folchetti, Camila Risso Barros, \\ Antonela Siqueira-Catani, Emanuel Péricles Salvador, \\ Sandra Roberta Gouvea Ferreira \\ School of Public Health/University of São Paulo
}

Background: The rs9939609 SNP of FTO (T > A) has been associated with obesity and its comorbidities. In carriers of the A allele, physical activity (PA) minimizes the deleterious impact on body weight, which could reduce cardiovascular risk. How sedentary behavior influence this association is unknown.

Objectives: To investigate whether PA level and the time spent watching television modulate the effects of A allele of the FTO on adiposity and markers of inflammation in individuals at high cardiometabolic risk.

Methods: This cross-sectional study included 158 prediabetic individuals (103 women, $54.7 \pm 12.5$ years, BMI $30.5 \pm 5.6 \mathrm{~kg} / \mathrm{m}^{2}$ ). Physical activities were measured by the long version of IPAQ, individuals were genotyped and stratified according to total PA $(<150$ or $\geq 150 \mathrm{~min} /$ week) and to time spent watching television $(<15$ or $\geq 15$ hours/week). Anthropometric, biochemical and inflammatory data were obtained. Subgroups of individuals with or without A allele were compared by student's $t$ test and Pearson coefficient used to test correlations.

Results: Among individuals who watched TV $<15$ hours/week, those carrying the A allele had significantly higher concentrations of total and LDL-cholesterol, apolipoprotein B $(88.3 \pm 27.0$ vs $96.8 \pm$ $21.1 \mathrm{mg} / \mathrm{dL})$ and interleukin-6 $(2.37 \pm 2.06$ vs $3.54 \pm 3.07 \mathrm{pg} / \mathrm{mL})$, but did not differ regarding anthropometric measures. Among those who watched TV $\geq 15 \mathrm{hrs} /$ week, no difference in any clinical data was detected comparing carriers and non-carriers of A allele. Regarding total PA, the presence of the variant allele did not influence the metabolic profile. In A allele carriers, time watching TV was correlated to
CRP $(r=0.227, p=0.039)$, reaching borderline significance for BMI $(\mathrm{p}=0.187, \mathrm{p}=0.09)$.

Conclusions: The presence of the A allele at FTO gene seems to favor a deleterious metabolic and inflammatory profiles particularly for individuals less exposed to sedentary activities such as watching TV. For inactive individuals, the presence of this allele might not affect predisposition to adiposity-induced disturbances.

\section{P168 \\ Ginkgo Biloba Extract (EGB) Increases AKT Phosphorylation in Retroperitoneal Fat Depot of Diet-Induced Obese (DIO) Rats}

\author{
Renata Mancini Banin, Bruna Kelly Sousa Hirata, \\ Caio César Sousa Ribeiro, Samira Alves Castiglioni, \\ Gabriella A. Alves Marian, Iracema Senna Andrade, \\ Juliane C. Silva Zemdegs, Ana Paula Grotti Clemente, \\ Ana Paula Dornelas Segantine, Lila Missae Oyama, \\ Cláudia Maria Oller Nascimento, Eliane Beraldi Ribeiro, \\ Monica Marques Telles
}

UNIFESP - Universidade Federal de São Paulo

Introduction: Consumption of high-fat diets is considered a risk factor for obesity and related co-morbidities among which stands out peripheral insulin resistance. It has been suggested that long-term high-fat intake may affect the insulin signaling cascade, impairing the translocation of GLUT4 (glucose transporters) to the cell surface and thus reducing glucose uptake by insulin-dependent tissues. We have previously demonstrated that EGb significantly reduced food intake and body adiposity and improved both insulin sensitivity and lipid profile of DIO rats. In addition, other studies have suggested that EGb761 therapy might be efficient to treat diabetes mellitus type 2. In this context, the aim of the present study was to evaluate the effect of prolonged treatment with EGb on Akt phosphorylation in retroperitoneal fat depot and gastrocnemius muscle of DIO rats.

Methods: Wistar rats were fed from 2 to 4-mo-old with high-fat diet (obese groups) or standard chow (Control group - C). After that, obese rats were divided in 2 groups: obese rats daily gavaged with saline $(\mathrm{O})$ and obese rats gavaged with $500 \mathrm{mg} / \mathrm{kg}$ of EGb (OG) during a 14-day period treatment. $\mathrm{C}$ group was gavaged with saline. After that, rats were anesthetized and the abdominal cavity was opened, the portal vein was exposed and $6 \mu \mathrm{g}$ of insulin was injected. Retroperitoneal fat pad and gastrocnemius muscle were removed before and 90 seconds after the infusion of insulin. The quantification of Akt phosphorylation was performed by Western blotting technique.

Results: Prolonged administration of EGb significantly increased insulin-stimulated Akt phosphorylation levels in retroperitoneal fat pad by $28.6 \%(p=0.009)$ in comparison to $O$ group. In gastrocnemius muscle, insulin infusion tended to increase Akt phosphorylation levels in OG group $(\mathrm{p}=0.052)$ while in O group Akt phosphorylation levels remained unaltered.

Conclusions: Ginkgo biloba therapy stimulated Akt phosphorylation in retroperitoneal fat depot of DIO rats. Those results combined to our previous finding which reported a significant improvement of 
insulin sensitivity suggest that this herbal medicine might be efficient as a hypoglycaemic therapy. Other studies are needed to better understand the effects of this phytotherapic medicine on insulin sensitivity.

Financial support: FAPESP (2011/06375-0).

P169

Effect of Isocaloric Intake of a High-Fat Diet on Adiposity, Lipid Profile and Adipokines Gene Expression in White Adipose Tissue from Rats

Monica Yamada, Marina Norde, Maria Carolina Borges, Miriam Helena Fonseca-Alaniz,

Maria Isabel Cardoso Alonso-Vale,

Francisco Leonardo Torres-Leal,

Tatiane Mieko De Meneses Fujii, Patrícia Silva Jacob,

Lucas Carminatti Pantaleão, Silvana Bordin, Rui Curi,

Julio Tirapegui, Primavera Borelli, Ricardo Ambrósio Fock, Marcelo Macedo Rogero

São Paulo University

Background: High-fat diet (HFD) is a relevant risk factor for the development of metabolic diseases. Studies have shown that hyperphagia induces body weight gain, leading to an increase in body fat deposition in rodents and humans. Obesity is associated with a state of chronic and low-grade inflammation, which is characterized by abnormal cytokine production, increased acute-phase reactants, and activation of inflammatory signaling pathways.

Objectives: The aim of this study was to investigate the effect of isocaloric intake of a HFD on adiposity, lipid profile and adipokines gene expression in periepididymal adipose tissue from rats. .

Material and Methods: Two months old male Wistar rats were randomly assigned to one of two groups: control (CON) group $(9 \%$ of calories from fat) and the HFD group ( $60 \%$ of calories from fat) $(n=6$ /group). After 12 weeks, body lipid composition was measured by carcass analysis. Serum lipid profile and C-reactive protein (CRP) were measured using commercial kits. Periepididymal adipose tissue was used to verify the expression of tumor necrosis factor (TNF)- $\alpha$, monocyte chemotactic protein (MCP)- 1 and adiponectin genes by Real-time PCR analysis. Prior to analysis, results were checked for distribution normality and homoscedasticity, and means from the control and HFD groups were compared by unpaired Student's t-test.

Results: Food intake (g) in the HFD group was lower in comparison to the CON group, however there were no differences in energy intake between the groups $(\mathrm{p}<0.05)$. Animals fed a HFD increased periepididymal $(p<0.05)$ and retroperitoneal $(p<0.05)$ fat depots and total body lipid content $(\mathrm{p}<0.05)$. The consumption of HFD increased serum LDL and total cholesterol levels $(\mathrm{p}<0.05)$, but not modified HDL, VLDL-cholesterol and triacylglycerols levels. The HFD group showed increased serum CRP level in comparison to the CON group $(p<0.05)$. However, there were no statistically differences in TNF- $\alpha$, MCP-1 and adiponectin gene expression between the groups. Conclusions: Dietary fat content, independent from caloric intake, induced an increase in adiposity and impaired lipid metabo- lism, without changes in adipokines gene expression in intra-abdominal white adipose tissue.

Financial support: FAPESP (09/50269-0) and $\mathrm{CNPq}$ (470418/2009-5).

\section{P170 \\ Determination Cell Free Plasma DNA in Breast Cancer Patients: Comparison Between Genes Encoding GAPDH and B-Actin}

Catarina M.N.O. Sediyama, Luís Fernando S. Moraes, Sérgio O. De Paula, Andreia Q. Ribeiro,

Maria do Carmo G. Peluzio

Federal University of Viçosa

Breast cancer is a major cause of morbidity and mortality among women worldwide. In developed countries, the increase in its incidence is accompanied by a decrease in its mortality However, in developing countries, such as Brazil, the high incidence is still accompanied by increasing mortality. The worldwide primary screening method used is the clinical breast exam and mammography. The discovery of cell-free DNA (CFDNA) in peripheral blood and its correlation with breast cancer raised the possibility of its use as a diagnostic tool in women with this condition. The aim of this study was to evaluate the use of two markers genes encoding glyceraldehyde3 -phosphate dehydrogenase (GAPDH) and $\beta$-actin for the quantification of plasmatic CFDNA in women with breast cancer (GCA) and healthy controls (GCO) in order to compare the diagnostic ability of the method and identify the best marker to classify patients with breast cancer. We evaluated 50 women, 30 cases and 20 healthy controls. It was performed CFDNA's plasmatic dosage using real time PCR. The association between several independent variables and the presence of breast cancer was evaluated by Student's t test, MannWhitney and chi square test, with a significance level of $5 \%$ to reject the null hypothesis. We estimated the odds ratio and its confidence intervals. The results showed that both $\beta$-actin and the GAPDH CFDNA was increased in plasma of women with breast cancer and controls, but the $\beta$-actin identified more accurately the patients with breast cancer. The CFDNA's concentrations found using the $\beta$-actin gene were lower in GCO and higher in GCA, with statistically significant difference between them $(\mathrm{p}<0.000)$. The dosage of CFDNA using $\beta$-actin was the best marker for identifying patients with breast cancer compared to the healthy control group. 


\section{P171 \\ Vitamins Levels in Women with Recurrent Miscarriages and DNA Methylation in IFNG Gene Promoter}

Nathalia Sierra Monteiro, Jessica Carrilho Britto,

Kelma Cordeiro S. Giusti, Mario Henrique B. Carvalho,

Antonio Amorim Filho, Marcelo Zugaib,

Elvira Maria Guerra-Shinohara

University of Sao Paulo (USP)

Background: Folate and cobalamin are vitamins involved in the cycle of remethylation of homocysteine to methionine, which is the precursor of S-adenosyl methionine, universal donor of methyl groups for DNA methylation, an epigenetic event that can regulate gene expression. IFNG encodes the cytokine interferon gamma and is regulated by epigenetic changes in promoter site. Disturbances in IFNG could lead to a change in cytokines balance, needed for fetal tolerance and lead to recurrent miscarriage (RM), defined by the occurrence of at least 3 spontaneous consecutive abortions until 22th week.

Objectives: To access DNA methylation in IFNG and SFN genes in women with primary RM (only abortions), secondary RM (at least one viable fetus) and in healthy women with at least 2 viable fetuses without known miscarriages and compare to folate, cobalamin (Cbl) and homocysteine (hcy) concentrations.

Methods: Fifty one women with history of primary RM, 35 women with secondary RM and 91 healthy women with normal deliveries were included. DNA was extracted of leukocytes and DNA methylation was detected by qMSP after bissulfite conversion. Cbl and folate were measured by microbiological method and Hcy by HPLC.

Results: No difference was found in DNA methylation between groups. DNA methylation in IFNG and SFN showed a strong correlation (pRM: $r=0.895 \mathrm{p}<0.001$, sRM: $r=0.883, \mathrm{p}<0.001$, Control: $\mathrm{r}=0.805, \mathrm{p}<0.001)$. IFNG methylation variability was influenced inversely by $\mathrm{Cbl}$ in primary $\mathrm{RM}(\mathrm{p}=0.046)$, directly by Hcy in secondary RM $(p=0.004)$ and inversely by folate in control $(p=0.032)$. SFN methylation variability was influenced inversely by $\mathrm{Cbl}(\mathrm{p}=$ 0.018 ) in primary RM and directly by Hcy in secondary RM (p = 0.007).

Conclusions: DNA methylation in IFNG and SFN genes is influenced by folate, $\mathrm{Cbl}$ and Hcy levels, although there was no difference between groups.

Ethical approval by Ethical Committee of Pharmaceutical Sciences.

Financial support: CNPQ, FAPESP.

\section{P172 \\ Relationship Between VDR Gene Polymorphisms and the Components of Metabolic Syndrome}

\author{
Natielen Jacques Schuch, Vivian Cristina Garcia, \\ Sandra Roberta Ferreira Vivolo, Lígia Araújo Martini \\ Faculdade de Saúde Pública - USP
}

Background/Aims: The vitamin D receptor gene (VDR) is expressed in many tissues and when it is in its activated form modulates the expression of several genes. The aim of the present study was to investigate the potential association between metabolic syndrome (MetSyn) with the presence of FokI or BsmI polymorphisms in VDR gene in Brazilian adult.

Methods: In a cross-sectional study, 243 Brazilian adult individuals were included. MetSyn was classified using the criteria proposed by NCEP-ATP III. Insulin resistance and $\beta$ cell secretion were estimated by calculating HOMA IR and HOMA $\beta$, respectively. The BsmI (A/G rs1544410) and FokI (C/T rs2228570) polymorphisms were detected by enzymatic digestion with specific enzymes and confirmed by allele specific PCR (ASPCR) or amplification of refractory mutation (ARM) in individuals with or without MetSyn.

Results: In patients with MetSyn, there is no association among BsmI polymorphism and components of MetSyn, HOMA IR and $\beta$, 25(OH)D3, and PTH. However, subjects without MetSyn, but with homozygosis for BsmI polymorphism (recessive bb genotype), presented lower levels of $25(\mathrm{OH}) \mathrm{D} 3$ than those with normal BB genotype. In addition, individuals with MetSyn and heterozygosis for FokI polymorphism (Ff genotype) have higher concentrations of PTH and HOMA $\beta$ than those with normal FF genotype. In this same group, subjects with the recessive ff genotype have higher insulin resistance than those with Ff genotype. On the other hand, patients without MetSyn, but carrying the Ff genotype, have higher concentration of triglycerides and lower levels of HDL than those with FF genotype. Interestingly, the presence of one allele $\mathrm{f}$ in the genotype ( $\mathrm{Ff}$ or $\mathrm{ff}$ ) is apparently enough to increase triglycerides levels and insulin resistance, when compared to the normal FF genotype.

Conclusions: The results show that FokI polymorphism in the VDR gene is associated to insulin resistance and higher concentrations of PTH in patients with MetSyn. Moreover, BsmI polymorphism is related to a lower concentration of $25(\mathrm{OH}) \mathrm{D} 3$ in individuals without MetSyn. Therefore, the results indicated that VDR gene polymorphisms are associated to different phenotypes of MetSyn components. 


\section{P173 \\ Brown and Golden Flaxseed Effects on Body Composition, Lipid Profile and Inflammation in Overweight Adolescents}

\author{
Adriane Moreira Machado, Janayra Maris Teixeira, \\ Marjorie Ostrowski, Heberth de Paula, \\ Luciane Daniele Cardoso, Neuza Maria Brunoro Costa \\ Federal University of Espirito Santo
}

Background: The comparative effect of golden and brown flaxseeds on lipid and inflammatory profile in overweight subjects has not been reported in the literature.

Objectives: The objective of the study was to evaluate the effects of golden and brown flaxseeds on lipid profile, inflammation and body composition of overweight adolescents.

Methods: 75 adolescents (33 boys and 42 girls), from 10 to 18 years of age and BMI/age $>$ score- $z+1$ and $<$ score- $z+2$, were recruited. The volunteers were divided into 3 groups $(n=25)$ and received $28 \mathrm{~g}$ of brown flaxseed (BF), golden flaxseed (GF) or wheat bran (Control - CG) from Monday to Friday for 11 weeks. Anthropometric and biochemical (lipid profile and C-reactive protein) analyses were performed before and after intervention. The normal distributed data were analyzed by one-way ANOVA and pared t-test while the non-parametric data were submitted to Wilcoxon Signed and Kruskal-Wallis tests, by using SPSS, version 19.0.

Results: 61 volunteers concluded the study $(\mathrm{BF}=20, \mathrm{GF}=20$, and $\mathrm{CG}=21)$. The mean daily intake of flaxseed was $14.4 \mathrm{~g}(\mathrm{BF})$ and $14.5 \mathrm{~g}(\mathrm{GF})$. No significant difference was observed between groups at baseline and the lipid profile and C-reactive protein did not change after intervention. The control group (CG) showed no variation in body weight, but there was a reduction on the percentage of fat after intervention, based on the measurement of triciptal and subscapular skinfolds. The flaxseed groups (BF and GF) showed significant increase on body weight and hight, but reduced body fat and no chance in BMI.

Conclusions: The benefits of flaxseed were not observed in the current study concerning lipid profile and C-reactive protein and no difference between brown and golden flaxseeds was found. Other inflammatory parameters are necessary to find out if the amount of flaxseed consumed is suficient to reduce the risk of chronic diseases in overweight adolescents, in association with the reduced body fat.

Financial support: CNPQ (472104/2010-1).

\section{P174 \\ Polyphenols from Propolis Reduces Matrix Metalloproteinase-9 Gene Expression and Activity in Activated Macrophages}

Nicolas Saavedra, Alejandro Cuevas,

Marcela Frota Cavalcante, Dulcineia Saes Parra Abdalla, Luis Antonio Salazar

University of São Paulo-Universidad De La Frontera

Background: Polyphenols from propolis has shown diverse biological activities such as anti-inflammatory and atheroprotective actions. In the context of atherosclerosis, macrophages have an important role, including the synthesis and secretion of matrix metalloproteinases and other enzymes involved in degradation of matrix extracellular components, influencing the stability of atherosclerotic plaques.

Objectives: To evaluate the in vitro effect of polyphenols from propolis on Mmp9 gene expression and proteolytic activity in LPS stimulated macrophages.

Methods: An ethanolic extract of polyphenols from Chilean brown propolis was prepared. To evaluate the cellular toxicity associated to these polyphenols MTT assay and apoptosis/necrosis detection by flow cytometry were used. These assays were performed using variable concentrations of extract and the highest vehicle concentration. RAW 264.7 cells were cultured and stimulated with LPS $(100 \mathrm{ng} / \mathrm{mL})$ to allow the cell activation and achieve an increment on Mmp9 production. To evaluate the in vitro activity of the extract on gene expression and protelytic activity, along with the activator, the cells were co-incubated with the corresponding treatment (extract or vehicle). Gene expression was quantified by qRT-PCR using relative quantification and the protelytic activity was measured by zymography.

Results: The highest non citotoxic concentrations to macrophages were identified as 10 and $7.5 \mathrm{ug} / \mathrm{mL}$ in the MTT and apoptosis/necrosis assays by flow cytometry, respectively. The total polyphenols extract promoted a dose-dependent significant inhibition on Mmp9 gene expression and gelatinolytic activity compared to the control $(\mathrm{P}<0.05)$.

Conclusions: Polyphenols from Chilean propolis showed a significant inhibition of Mmp9 gene expression and activity, suggesting a potential role in the control of extracellular matrix degradation in atherosclerotic plaques. 


\section{P175 \\ Effect of Bark Extract of Bathysa Cuspidata on Hepatic Oxidative Damage in Wistar Rats Induced to Pre-Neoplasic Lesions}

Patrícia Aparecida Fontes Vieira, Alessandra Barbosa Ferrei ra Machado, Natália Filardi Tafuri, Elisa Sialino Muller,

Letícia Linhares Da Silva, Ana Carolina Machado Fonseca, Nathane Pais Siqueira, Lisiane Lopes da Conceição, Maria do Carmo Gouveia Peluzio

Universidade Federal de Viçosa

Bathysa cuspidata (A. St. Hil.) Hook f. belongs to the Rubiaceae family and your bark extract has coumarins, flavonoids, tannins and alkaloids. These secondary metabolites appear to be responsible for the protective effects of the extract on the free radicals damages. It's well established that basal levels of reactive oxygen species (ROS) are elevated in chronic diseases, especially cancer (CA). In the endogenous antioxidant defense system, the enzymes superoxide dismutase (SOD) and catalase (CAT) act scavenging ROS. This study evaluated the ethanolic extract effect of B. cuspidata on the activity of SOD and CAT in the liver of rats submitted with the carcinogen 1.2 dimethylhydrazine $(\mathrm{DMH})$, also hepatotoxic enzymes such as aspartate aminotransferase (AST) and alanine aminotransferase (ALT) were analyzed in serum of animals. The powdered stem bark of Bathysa cuspidata (600 g) was exhaustively extracted by percolation with etanol $95 \%$. Freeze dried extract was administered by gavage to the animals on alternate days for 70 days. Rats with 9 weeks old were divided into 4 groups: G1 (non-induced treated with $400 \mathrm{mg} / \mathrm{kg}$ extract), G2 (induced with pre-neoplasic colorectal lesions treated with $200 \mathrm{mg} / \mathrm{kg}$ extract), G3 (induced with pre-neoplasic colorectal lesions treated with extracts $400 \mathrm{mg} / \mathrm{kg}$ ) and G4 (non-induced treated with saline). Were used the spectrophotometric method for the data collection activity of SOD and CAT at $510 \mathrm{~nm}$ and $240 \mathrm{~nm}$, respectively. Analysis of ALT and AST enzymes were performed using kits. Data were analyzed using ANOVA, and means tested by Tukey test at $5 \%$ of significance. An activity (U/mg protein) of SOD in G2 was significantly higher $(\mathrm{P}<0.001)$ when compared to other groups. The CAT from $\mathrm{CA}$ induced groups treated with extract was significantly higher compared with $\mathrm{G} 1$ and $\mathrm{G} 4(\mathrm{P}=0.002)$. There was no change in AST enzyme activity $(P=0.05)$ and ALT activity increase significantly in groups with pre-neoplasic colorectal lesions and treated with the extract $(\mathrm{P}=0.006)$. The AST reduced activity in serum suggests liver protection. This type of investigation has been shown to represent an effective strategy for selecting medicinal plants for the development of new drugs, as part of the world population uses products derived from plants in their basic healthcare.

Financial support: CAPES / FAPEMIG.

\section{P176 \\ Gestacional Protein Restriction Induces Dendritic Atrophy in Dorsal Hippocampal Neurons and Neurochimical Changes But Does Not Alter Learning and Memory Performance in Adult Offispring}

\author{
Agnes Silva Lopes, Daniele Braz Torres, \\ Ana João Rodrigues, João José Cerqueira, \\ José Miguel Pêgo, José Antonio R. Gontijo, Nuno Sousa, \\ Patrícia Aline Boer \\ UNESP
}

Background: Studies have demonstrated that maternal nutritional restriction during pregnancy or in early postnatal life results in structural abnormalities in the offspring hippocampus and cognitive impairment.

Objectives: To analyze whether gestational protein restriction might induce learning and memory impairment associated with structural and neurochemistry changes in the hippocampus.

Methods: Virgin female Wistar rats were fed during pregnancy with normal-protein diet (control - 17\% casein) or protein-restricted diet (LP 6\% casein). In male offspring with 16-wk old we carried out Morris Water Maze (MWM) test and a detailed morphometric analysis of dendritic cytoarchitecture of the hippocampus. In addition, we analyzed by western blot and immunohistochemistry the dorsal and ventral hippocampal expression and localization of mineralo- (MR) and glucocorticoid (GR), type 1 angiotensina II receptor (AT1) and serotonin receptors (5HT1A and 5HT2A).

Results: By MWM we did not found significant differences between LP and NP groups, in any of the parameters analyzed, suggesting that such functions of hippocampus were not altered by gestational protein restriction. However, by applying 3-dimensional analysis of dendrites from the dorsal hippocampus, this study demonstrates that gestational protein restriction leads to decreases in total basal dendritic length and in apical intersections of CA3 pyramidal neurons. The dendritic architecture of CA1 and dentate gyros was unchanged. We found different patterns of dorsal and ventral expression of analyzed receptors. MR expression was not altered but GR, 5HT1A and AT1 expression was reduced. We also found enhanced 5HT2A expression.

Conclusions: The current study revealed a clear dissociation between behavioral test response and hippocampal neuron changes as consequence of fetal programming. We suggest that reduced GR and 5HT1A and enhanced 5HT2A expression are involved in anxious behavior and that AT1 down regulation may have a protective effect. These neurochemical alterations may have important consequences for anxiety- and depressive-like behavior. 


\section{P177}

\section{Gestacional Protein Restriction Triggers Hyperanxiety in Adulthood Due to Changes in the Bed Nucleus of the Stria Terminalis (BNST)}

\author{
Daniele Braz Torres, Agnes Silva Lopes, \\ Ana João Rodrigues, João José Cerqueira, \\ José Miguel Pêgo, Antonio Francisco Godinho, \\ Nuno Sousa, José Antonio R. Gontijo, Patricia Aline Boer \\ UNESP
}

Background: The BNST is located in a key position to regulate stress response and has been implicated in anxiety behavior. Evidence suggests that this brain region is highly plastic and responsive to deleterious stimuli in terms of neuronal morphology.

Objectives: Investigate in adult rats submitted to protein restriction in utero compared with controls: behavioral parameters, 3D dendritic structure in neurons and the neurochemistry compounds of BNST.

Methods: Virgin female Wistar rats were fed during pregnancy with normal-protein diet (control $-17 \%$ casein) or protein-restricted diet (LP 6\% casein). In male offspring with 16-wk old we carried out open field and elevated plus maze tests. The brains were processed for Golgi-Cox staining Three-dimensional reconstructions of representative Golgi-impregnated neurons from the BNST. Western Blot was used to evaluate in isolated BNST, the expression of 11- $\beta$ HSD2, AT1 (angiotensin II receptor type 1), 5 HT1A, 5 HT2A (serotonin receptors), MR (mineralocorticoid receptor) e GR (glucocorticoid receptor).

Results: LP offspring presented significant reduction in dendritic tree length in BNST neurons in parallel to the rise in the expression of ansiogenic receptors and reduction in ansiolytic. Additionally, the elevated plus maze analyze showed elevated anxiety-like behavior.

Conclusions: Our results demonstrate for the first time that gestational protein restriction can induce significant morphological and neurochemical changes in the BNST that can be related to an anxious behavior in the progeny.

\section{P178 \\ Indicated for the Young Investigator Award Involvement of Glomerular Micro-RNAs Expression on Epithelial-to-Mesenchymal Transition in Adult Maternal Low Protein Diet Programmed Rats}

\author{
Letícia Barros Sene, Flávia Mesquita Vieira, \\ Leonardo Nazário Moraes, Robson Francisco Carvalho, \\ José Antônio Gontijo, Patrícia Aline Boer
}

Universidade Estadual Paulista "Júlio de Mesquita"

Background: Nutritional restriction may result in intrauterine growth retardation associated with impaired nephrogenesis and nephron underdosing. Recently, study has been show that maternal protein-restricted (LP) 16-wk-age offspring, present pronounced reduction of nephron number associated with decreased fractional urinary sodium excretion and arterial hypertension.

Objectives: To investigate the effects of maternal low protein diet exposure in utero on glomerular function, ultrastructure and the expression of miRNAs, mRNAs and proteins involved in fibrosis and epithelial-to-mesenchymal transition (EMT).

Methods: Virgin female Wistar rats were fed during pregnancy with normal-protein diet (control $-17 \%$ casein) or protein-restricted diet (LP 6\% casein). In male offspring with 16-wk old was evaluated the protein urinary excretion rates. Transmission and scanning electron microscopy were used to characterize the glomerular ultrastructure. Glomeruli were isolated to investigate the expression of miR 200 family and miR 192 and mRNAs (col $1 \alpha 1$ e 2, desmin, E-caderin, fibronectin, TGF- $\beta 1, \mathrm{ZEB} 1 / 2$ and ZO-1) by real time-PCR. Proteins markers of fibrosis and epithelial-to-mesenchymal transition (EMT) were studied by western blot and immunohistochemistry.

Results: LP rats presented heavy proteinuria associated to podocytes simplification and foot process effacement, increased thickness of glomerular basement membrane. Isolated glomeruli shows reduced level of miR-200a, miR-141 and miR-429 in LP offspring and miR200c and miR-192 expression are not altered. ZEB2 mRNA was upregulated in glomeruli from LP rats. Collagen $1 \alpha 1 / 2$ mRNA was upregulated but the other mRNAs for EMT markers studied are not altered. The glomeruli shows a reduced immunoreactivity to nephrin and enhanced to TGF- $\beta 1$, fibronectin, and collagen type I in LP rats. By western blot analyses of total kidney tissue we found significant reduction of both podocin and nephrin and enhanced TGF- $\beta 1$ expression in LP rats.

Conclusions: The fetal programmed adult rats presented a striking structural glomerular disorder with an accentuated and advanced stage of fibrosis that led us to state that glomerular miR200 family would be early upregulated by TGF- $\beta 1$ action inducing type I collagen expression. In this way, the increased renal collagen expression may subsequently cause glomeruli EMT by decreasing these miRNAs at later time points.

\section{P179 \\ Expression of Cell Adhesion Molecules in Atherogenesis: an Analysis of the Interfering Factors}

Patrícia Feliciano Pereira, Franciane Rocha Faria, Maria Do Carmo Gouveia Peluzio, Sylvia do Carmo Castro Franceschini, Silvia Eloiza Priore

Federal Univervisity of Viçosa

Atherogenesis may start during fetal life and its course consists of inter-relationships between traditional risk factors and new biomarkers. It has been suggested that atherosclerosis is a specific form of chronic inflammation. This review was focused on vascular (VCAM1) and intercellular (ICAM-) cell adhesion molecules, markers whose crucial roles in all phases of the development of atherosclerosis still need to deepen the scientific knowledge. These molecules mediate adhesion and migration of monocytes to arterial intima, the early stages of atherogenesis. They are expressed by monocytes, macrophages, $\mathrm{T}$ and $\mathrm{B}$ lymphocytes, fibroblasts, epithelial and endothelial 
cells. Longitudinal studies document as a predictor of cardiovascular disease, high circulating levels of ICAM-1 and VCAM-1, the latter being less consistently. Lifestyle factors and genetic induce the secretion of these molecules. Being obese and male gender was associated with higher levels of ICAM-1. The level of these markers appears to reduce with age, which suggests caution in interpreting values. Regarding diet, there was an inverse association between diet and VCAM-1, and direct link between western pattern with ICAM-1 and VCAM-1. Investigators from the Nurses' Health Study confirmed these findings, but other studies haven't observed these differences. In a recent study in mice with diet rich in cholesterol levels were increased ICAM-1 but not VCAM-1. The type of dietary fatty acids is also an influence factor, once the palmitic acid in human activated expression of adhesion molecules while linoleic acid reduced. The garlic extract has shown in vitro to be able to modulate the expression of ICAM-1 and VCAM-1. Alcohol use, smoking and physical inactivity are associated with higher levels of adhesion molecules. Some genetic polymorphisms have also been identified and appear to influence the circulating levels of these molecules, including G241R, $\mathrm{K} 469 \mathrm{E}$, rs5496, rs5498, rs3093030 and rs1799969. It is known that TNF-alpha, fibrinogen, IL-1, IL-4, interferon- $\gamma$, angiotensin II, LDL and the transcriptional factor NF-kappaB modulate the expression of adhesion molecules. The adhesion molecules may add prognostic information to traditional risk factors and allow for early identification of atherosclerosis, allowing for primary prevention that is primordial and should be encouraged.

Financial support: CNPQ and FAPEMIG.

\section{P180 \\ Omega-3 in the Regulation of Non-Esterefied Fatty Acids (NEFa) and the Expression of the Lipogenics Transcription Factors}

\author{
Patrícia Mattei, Carolina Bespalhok Jacometo, \\ Eduardo Schmitt, Augusto Schneider, \\ Luiz Francisco Machado Pfeifer, \\ Nelson José Laurino Dionello, Francisco Burkert Del Pino, \\ Marcio Nunes Corrêa \\ Universidade Federal de Pelotas
}

Background: The polyunsaturated fatty acids (PUFAs) are essential for the tissues development, and their supplementation during pregnancy can induce some alterations on the pregnant and offspring lipid metabolism, at biochemical and gene expression levels.

Objectives: To evaluate if a diet rich in PUFAs can regulate pre and postpartum NEFA concentrations and the effect on the expression of lipogenic transcription factors in consecutive generations.

Methods: Eighteen female Rattus norvegicus - Wistar/UFPel, 8 weeks old, were separated in two groups: 1) diet rich in omega-6, with soybean oil (CTL) and, 2) diet rich in omega-3, with flaxseed oil (OM). The diets were elaborated in accordance with AIN-93 recommendations. After 30 days of adaptation the rats were mated. The males received only the control diet. The F1 females were selected at weaning ( $\mathrm{n}=16 /$ group) and divided into three groups: females from OM group that continued receiving the same diet (OM-OM), females from OM group that began to receive diet containing soybean oil
(OM-CTL), and females from CTL group that continued to receive the same diet (CTL-CTL). These animals were mated at 60 days old as described (G0). The F2 ( $\mathrm{n}=16 /$ group) was selected as F1 and the same groups were maintained: OM-OM-OM, OM-CTL-CTL, and CTL-CTL-CTL. The F2 was mated as F1. Female pre-partum (19 days of pregnancy, $n=4 /$ group) and postpartum euthanasias (21 days, $n=6 /$ group) were conducted for each generation to collect blood and liver samples. NEFA concentrations were measured by colorimetric method. Real-Time quantitative PCR was performed to evaluate the liver $\mathrm{X}$ receptor alpha $(\mathrm{LXR} \alpha)$ and sterol regulatory element binding protein 1c (SREBP-1c).

Results: Comparing NEFA postpartum concentration between the generations, the OM group maintained a constant, while the CTL group had a decrease in concentration from G0 to F1 $(\mathrm{P}=0.04)$ and increased from $\mathrm{F} 1$ to $\mathrm{F} 2(\mathrm{P}=0.005)$. However, no differences were observed between groups in the prepartum period. LXR $\alpha$ pre-partum expression increased from F1 to F2 (CTL, P < 0.001, OM-CTL, P < 0.001 ), except to the OM group, and postpartum expression increased in all groups across generations $(\mathrm{P}<0.001)$. SREBP-1c expression increased for all groups, both pre and postpartum, from F1 to F2 (P < 0.001).

Conclusions: PUFAs had an effect on the plasmatic NEFA concentrations and a cumulative effect across generations at the expression of transcription factors related to lipogenesis.

\section{P181 \\ Influence of GPX4 in Male Infertility \\ Priscila Pereira Pessoa, Christielle Félix Barroso, Luana Mara Silva Castro, Alana Flávia Fernandes Santos, Larissa Bezerra Santos, Carla Soraya Costa Maia \\ Universidade Estadual do Ceará}

The reactive oxygen species (ROS) have pathophysiological importance in the etiology of male infertility. The human sperm present the major antioxidant defense systems the phospholipid hydroperoxide glutathione peroxidase (GPx4), which directly reduces oxidized phospholipids. GPx4, which has selenium (Se) on its active site, is found in the mitochondria of the testis and sperm. The articles cited in this literature review were indexed in the databases PubMed, Scielo, Science Direct between the years 2008 and 2012, using the following keywords: GPx4, male infertility, mice, selenium. Schneider et al. (2009) demonstrated that the GPx4 disorders caused a severe structural impairment in the middle piece of rats spermatozoa, arguing that this selenoenzyme confers the vital role of selenium in male fertility in mammals. Study of Imai et al. (2009) demonstrated that GPx4 depletion of mice spermatocytes resulted in infertility. Both sperm motility and mitochondrial membrane potential showed significantly reduction, which are accompanied by structural changes in the middle piece of spermatozoa. A study by Gautrey et al. (2011) showed that $\mathrm{T} / \mathrm{C}$ polymorphism in the 3'UTR region of the GPx4 gene affects the expression of selenoprotein and the viability of cells transfected into Caco-2 cells. When Se supply was low, cells overexpressing the $\mathrm{C}$ variant 3'UTR showed lower viability after oxidative stimulation, increased levels of ROS, lower GPx activity and mRNA expression compared with cells overexpressing the T variant. After Se supple- 
mentation, cell viability and GPx4 expression were higher in cells overexpressing the $\mathrm{C}$ variant. A more recent study of Atig et al. (2012) evaluated the antioxidant profile in men with idiopathic infertility, which showed a significant association of sperm motility in the presence of Se in seminal fluid and an antioxidant action impaired by Se deficiency. We conclude that depletion of GPx4 causes severe abnormalities in sperm and may be a cause of infertility in rats and humans. Therefore, higher intake of Se leads to high expression of the selenoprotein, being a protective factor.

\section{P182 \\ Perinatal Undernutrition and Fluoxetine: Gene Expression Analysis of 5HT-1a, MAO-A e BDNF in the Hippocampus of Adult Rats}

\author{
Priscilla M. Ferreira Vieira, Matheus Cavalcanti Pinho, \\ Tercya Lucidi de Araújo Silva, Ricardo Orozco Solis, \\ Sandra Lopes Souza, Raul Manhães de Castro, \\ Rhowena Jane Barbosa de Matos \\ Universidade Federal de Pernambuco CAV/ UFPE
}

Background: The undernutrition during the period of gestation and lactation alters the developing process of the offspring that could results in cognitive deficiencies of learning memory. In these processes of neuronal plasticity participates both, the serotoninergic receptors 5HT-1A and the neurotropic factors BDNF and IGF-1. These genes are overexpressed in response to antidepressants such as fluoxetine an inhibitor of the serotonin reuptake .

Objectives: To determine the long-lasting effects of protein undernutrition in the expression of the genes 5HT-1A, MAO-A, BDNF and IGF-1 in the hippocampus of adult rats in response to fluoxetine.

Methods: Wistar rats (90-100 days) were divided according with the received diet during the gestation and lactation until weaning as follows: control $(\mathrm{C}$, casein $17 \%, \mathrm{n}=3)$ undernourished $(\mathrm{D}$, casein $8 \%, \mathrm{n}=3$ ). Offspring males were divided at 158 days of life according with the treatment received during 22 days: (CF and DF, fluoxetine $10 \mathrm{mg} / \mathrm{Kg}$, s.c., $\mathrm{n}=5$ ) or saline (CS and DS, $0.9 \% \mathrm{NaCl}$, s.c., $\mathrm{n}=$ 5). At 180 days rats were scarified by decapitation and RNA were extracted from hippocampus and real-time PCR were used to quantify the expression of the genes $5 \mathrm{ht}-1 \mathrm{a}$, mao-a, bdnf e igf- 1 and b-actin as housekeeping gene. Relative gene expression were calculated with the 2- $\Delta \Delta$ CT method, averages were compared by two-way ANOVA, $\mathrm{P}<0.05$. Results, we found significant interaction diet $\mathrm{x}$ treatment in the undernourished animals that received chronic injection of fluoxetine, showing an increase in the gene expression of the genes: 5 ht$1 \mathrm{a}, 40 \%(\mathrm{CS}=1.00 \pm 0.03, \mathrm{CF}=1.21 \pm 0.15, \mathrm{DS}=1.00 \pm 0.08, \mathrm{DF}=$ $1.40 \pm 0.21, \mathrm{p}<0,05, \mathrm{n}=5)$, mao-a, $30 \%(\mathrm{CS}=1.01 \pm 0.13, \mathrm{CF}=$ $1.03 \pm 0.14, \mathrm{DS}=0.93 \pm 0.11, \mathrm{DF}=1.31 \pm 0.09, \mathrm{p}<0.001, \mathrm{n}=5)$, bdnf, $90 \%(C S=1.01 \pm 0.19, C F=0.71 \pm 0.20, \mathrm{DS}=1.01 \pm 0.19$, $\mathrm{DF}=1.92 \pm 0.32, \mathrm{p}<0.001, \mathrm{n}=5$ each group), and not alteration for igf-1 $(\mathrm{CS}=1.01 \pm 0.16, \mathrm{CF}=0.88 \pm 0.10, \mathrm{DS}=1.01 \pm 0.16, \mathrm{DF}=$ $0.91 \pm 0.10, \mathrm{p}>0,05, \mathrm{n}=5$ each group).

Conclusions: The stimulus by fluoxetine increase the serotoninergic activation in the undernourished animals, which suggests that these animals presents an intrinsic over activation of the serotoniner- gic system that could improve the mechanisms of hippocampal plasticity and memory formation. Implying that perinatal protein undernutrition may have an effect during the development of the serotoninergic system in the hippocampus. Additional experiments are being done to determine if these animals have improved memory capacities.

Financial support: FACEPE/ CNPQ (APQ-0087-4.05/10).

\section{P183 \\ Creatine Supplementation in Fatty-Liver Disease}

Rafael Deminice, René L. Jacobs, Margaret E. Brosnan, John T. Brosnan, Alceu Afonso Jordao

University of Sao Paulo

Background: Fat accumulation and the progression of nonalcoholic fatty-liver disease (NAFLD) have been associated to impaired hepatic methionine metabolism. Creatine is a methylated compound responsible for a considerable consumption of S-adenosylmethionine in the liver. Recently, creatine intake has shown to modulated methyl balance. However, the effect of creatine supplementation on fattyliver disease is unknown.

Objectives: Examine the effects of creatine supplementation on liver fat accumulation and dysfunction in methionine metabolism, induced by a high-fat diet in rats. We also examined the effects of betaine supplementation as a positive control.

Methods: 24 rats were divided into 4 groups: control diet (C), high-fat diet (HF), high-fat diet supplemented with betaine (HFB), and high-fat diet supplemented with creatine (HFC). The C and HF liquid diets contained, respectively, 35 and $71 \%$ of energy derived from fat. Betaine and creatine were supplemented in the diet $(1 \% \mathrm{wt} /$ vol) for 3 weeks.

Results: The HF diet significantly increased total liver fat concentration (25\%), liver triglycerides (37\%), liver TBARS (14\%) and decreased hepatic S-adenosylmethionine concentration (SAM) (18\%) and SAM/SAH (24\%). Betaine and creatine supplementation normalized these perturbations. As previously documented, betaine supplementation increased hepatic SAM (276\%) and phosphatidylcholine (PC) formation and prevented fat accumulation. Creatine supplementation greatly down-regulated endogenous creatine synthesis, as evident from a 90\% decrease in renal arginine:glycine amidinotransferase activity (AGAT). It also returned hepatic SAM levels to normal. However, increased liver phenylethanolamine (PE) and decreased the $\mathrm{PC} / \mathrm{PE}$ ratio were observed in HFC group compared to rats fed the other diets. Thus, we examined the abundance of genes involved in the metabolism of S-containing amino acids, fatty acids and phospholipids. Creatine normalized the expression of Gnmt and Bhmt. It also reduced Ppara mRNA levels as well as those of its downstream targets Cptla, Lcad and Cd36 genes of mitochondrial ß-oxidation, and fatty acids transporter, respectively.

Conclusions: Betaine and creatine supplementation prevented the fatty-liver induced by $\mathrm{HF}$ by different mechanisms. Betaine increased SAM availability and PC formation. Creatine normalized the expression of Gnmt and Bhmt and key genes of beta-oxidation. To 
our knowledge, this is the first demonstration of this effect of creatine.

Financial support: Supported by Cihr, Canada and FAPESP, Brazil.

\section{P184 \\ Maternal Consumption of High-Fat Diet During Pregnancy and Lactation Leads to Modulation of MicroRNA Expression Related to Lipid Metabolism}

Rafaela Oliveira Benatti, Arine Mattos Melo, Nicole Guimarães Ashino, Letícia Martins Ignácio-Souza, Lício Augusto Velloso, Márcio Alberto Torsoni,

Adriana Souza Torsoni

\section{Universidade Estadual de Campinas - UNICAMP}

Introduction: Obesity has been pointed as a ubiquitous disease in society that advances quickly in all races, genders, social levels and age groups. Children of mothers who consume high-fat diet during pregnancy and lactation accumulate lipids in the liver and activate inflammatory pathways that culminate in the development of insulin resistance in various tissues. Recent studies using a model of dietinduced obesity showed the relationship to microRNA expression and metabolic disturbance. The miR-122, an important microRNA related to lipid metabolism and highly expressed in liver, showed altered expression in obese individuals, concomitant to altered expression of genes involved to lipogenesis. It is really important to understanding whether the conditions for development of the fetus in obese mothers could contribute to the microRNAs activation that promote metabolic damage observed in the adult offspring.

Methodology: For the present study was used male Swiss mice (28 days), from dams that consumed either control diet (SC-O) or high fat diet (HFD-O) during pregnancy and lactation. After weaning, the offspring SC-O and HFD-O were fed with control diet for 7 days. Liver fragments were used to evaluate the expression of miR-122 using real time PCR (RT-PCR). Lipogenic proteins (FAS, SCD-1, HMGCR) were also investigated using RT-PCR and/or Western Blot technique (WB).

Results: HFD-O mice presented increased body weight $(7 \mathrm{~d})$ compared to SC-O. Furthermore, the results obtained by RT-PCR and WB showed higher HMGCR (1.5-fold to WB and 587-fold to RT-PCR), SCD-1 (1.3-fold WB and 708-fold to RT -PCR) and FAS (1.4-fold to $\mathrm{WB}$ and 86-fold to RT-PCR) expression in liver of HFD-O than SC-O mice. The liver expression of miR-122 in HFD-O was lower $(99.22 \%)$ than SC-O mice.

Discussion: The results of the present study showed that the consumption of a high fat diet during pregnancy and lactation lead to diminished expression of liver miR-122 in the offspring. This result was accompanied by increased expression of lipogenic genes. This molecular alteration may be related to the fatty liver observed in the adult offspring of dams fed with a high fat diet during pregnancy and lactation.

Financial support: FAPESP (2011/13947-0).

\section{P185 \\ Dietary Combination of Conjugated Linoleic Acid with Phytosterols Reduces Adipose Tissues in Lean and Obese Rats}

\author{
Rafaela da Silva Marineli, Cibele Priscila Busch Furlan, \\ Larissa G. Bajay, Mário Roberto Maróstica-Júnior \\ UNICAMP
}

It is established that adipokines play a role in the maintenance of an inflammatory state in adipose tissue and in the development of obesity and comorbidities. We investigated the effects of dietary CLA and phytosterols supplementation in adipose tissue weight and in leptin concentration in lean and obese rats. Seventy-five SpragueDawley rats were fed with high-fat diet (35\%) or normal-fat diet (9\%) supplemented with $2 \%$ of each lipid compound. Animals were divided into nine groups: Standard group $(\mathrm{P})$, Control high-fat group (HF), Control linoleic acid group (LA), three high-fat groups fed diets added CLA (HC), phytosterol (HP) and CLA plus phytosterols (HC + $\mathrm{P})$, three normal-fat goups fed diets added CLA (C), phytosterol (P) and CLA plus phytosterols $(\mathrm{C}+\mathrm{P})$ for 9-weeks. Animals were sacrificed by decapitation preceded by 12-h-fasting. Epididymal adipose tissue was removed and weighed. Blood samples were collected and serum leptin was analyzed using enzyme-linked immunosorbent assay method. The animals that received the combination of CLA and phytosterols showed a clear decrease on epididymal adipose tissue weight, $44 \%$ for obese and $35 \%$ for lean rats compared to control groups $(P<0.05)$. The serum leptin concentration in CLA groups was significantly lowered, $22 \%$ for obese and $60 \%$ for lean rats compared to control groups $(P<0.05)$. It is known that leptin concentration is directly associated with adiposity and an important role for adipose tissue as an endocrine organ. In summary these finds suggest that the dietary association between CLA and phytosterols acted in a complementary and synergistic way and could be beneficial for the prevention of metabolic syndrome by suppressing fat accumulation.

Financial support: FAPESP.

\section{P186 \\ Freeze-Dried Jaboticaba Peel (Myrciaria Jaboticaba (Vell.) Berg) Add to High-Fat Diet Ameliorate Insulin Resistance in Obese Rats}

\author{
Sabrina Alves Lenquiste, Ângela Giovana Batista, \\ Anne Castro Marques, Rafaela da Silva Marineli, \\ Mário Roberto Maróstica-Júnior
}

School of Food Engineering, University of Campinas

Jaboticaba is a typical fruit of the Brazilian cerrado, very rich in anthocyanins, which is attributed powerful effect antioxidant, antiobesogenic and combat insulin resistance. This study evaluated the effect of add freeze-dried jaboticaba peel (FJP) in high-fat diets in serum glucose, insulin and insulin resistance in rats. Thirty-five Sprague Dawley rats, divided in 5 groups were fed with the diets: Normal Control (NC) - diet AIN 93-G, High-Fat control (HF) - diet AIN 93-G with $35 \%$ fat (31\% lard and $4 \%$ soybean oil), HFJ1 - HF 
diet $+1 \%$ FJP, HFJ2 - HF diet + 2\% FJP, HFJ4 - HF diet + 4\% FJP. Supplemented groups were fed for 28 days with HF diet and for more 42 days with the diets with FJP. After the experiment, the animals were fasted for 12 hours and killed by decapitation. The blood was collected to obtain serum and the pancreas and epididymal adipose tissue (EAT) were removed and weighed. We analyzed the serum glucose and insulin by enzymatic and ELISA kit, respectively. The blood glucose and insulin were used to calculate HOMA-IR (Homeostasis Model Assessment). The percentage weight of the pancreas and EAT in relation to final body weight of animals was calculated. Pancreas showed no statistical difference between groups $(p>0.05)$, but the EAT showed the higher values in the HF group and significantly diminution in HFJ1. The serum glucose was significant lower $(\mathrm{p}<0.05)$ in CN. Serum insulin and HOMA-IR index were significantly lower $(p<0.05)$ in groups fed with FJP. We conclude that the consumption of FJP reduced hyperinsulinemia in animals, acting positively in prevention of the insulin resistance.

Financial support: FAPESP.

P187

PPAR Alpha and Gamma Modulation: FAS and CPT-2 Gene Expression in Liver and Adipose Tissue by Dietary Conjugated Linoleic Acid in Short and Long Supplementation Period in Healthy Rats

\begin{abstract}
Lilia Ferreira Santos-Zago, Adriana Prais Botelho, Rafaela da Silva Marineli, Sabrina Alves Lenquiste, Fernanda Seyr Pozza, Luiza Bernardi Pardo, Cibele Priscila Busch Furlan, Soely Maria P.M. Reis, Admar C. Oliveira, Mário Roberto Maróstica-Júnior
\end{abstract}

School of Food Engineering, University of Campinas

The effects of conjugated linoleic acid (CLA) on lipid metabolism have been explained by modulation of peroxissome proliferators-activated receptors (PPAR), a nuclear receptor family fatty acid-regulated that represents important regulators of genes. Two members of this family, PPAR alpha and PPAR gamma have critical role in regulation of lipolysis and lipogenesis by activating expression of genes involved in fatty acid oxidation and fatty acid biosynthesis such as carnitine palmitoyltransferase-2 (CPT-2) and fatty acid synthase (FAS) genes, respectively. The objective of this study was to evaluate the influence of dietary CLA supplementation on gene expression of PPAR alpha and gamma, FAS and CPT-2 in liver and adipose tissue of rats. Forty male healthy Wistar rats were divided into four groups $(\mathrm{n}=10)$ and received diet supplemented with $2 \%$ of a commercial mixture of $\mathrm{c} 9 \mathrm{t} 11$ and $\mathrm{t} 10 \mathrm{c} 12$ CLA Tonalin ${ }^{\circledR}$ isomers (EE) or $2 \%$ of safflower oil as placebo (EP). Euthanasia was carried out after $4(\mathrm{n}=$ $20)$ and 8 weeks $(n=20)$ of supplementation. Samples of liver and epididymal adipose tissue were immediately removed and frozen in liquid nitrogen for RNA extraction. Experiments were performed using real time polymerase chain reaction (RT-PCR) and a commercially available test system was used to determine the gene expression of PPAR and enzymes. After 4 weeks, there was an increase of gene expression of PPAR alpha ( $87 \%$ in liver and $63 \%$ in adipose tissue) and PPAR gamma (64\% in liver and $78 \%$ in adipose tissue) and a reduction of gene expression of FAS $(60 \%$ in liver and $75 \%$ in adipose tissue) and CPT-2 (83\% in liver and $60 \%$ in adipose tissue) in the group supplemented with CLA $(\mathrm{p}<0.05)$ compared to control. Furthermore, after 8 weeks, there was an increase of gene expression of PPAR alpha (65\% in liver and $134 \%$ in adipose tissue) and PPAR gamma ( $80 \%$ in liver and $159 \%$ in adipose tissue) and a reduction of gene expression of FAS (69\% in liver and $64 \%$ in adipose tissue) and CPT-2 (76\% in liver and $60 \%$ in adipose tissue) in the group supplemented with CLA $(\mathrm{p}<0.05)$ compared to control. It was concluded that the increased gene expression of PPAR alpha and gamma not necessarily resulted in increased expression of genes under the transcriptional control of PPAR, such as CPT-2 and FAS, suggesting that CLA can influence fatty acid oxidation and fatty acid biosynthesis by PPAR dependent and independent mechanisms in short and long supplementation period.

Financial support: FAPESP.

\section{P188 \\ Butyrate Attenuates Glomerular Injury in an Experimental Model of Focal and Segmental Glomerulosclerosis}

Raphael J.F. Felizardo, Rafael Luis Pereira, Clarice S.T. Origassa, Marcus Antonio Cenedeze, Vinícius Andrade Oliveira, Marco Aurélio Vinolo, Rui Curi, Alvaro Pacheco-Silva, Niels O.S. Câmara

Universidade Federal de São Paulo

Butyrate is a short-chain fatty acid produced by colonocytes through anaerobic fermentation of dietary non digested fibers, and immunologically important despite its well known properties in modulate immune response. Several studies show the capacity of butyrate in modulating intestinal and extra-intestinal inflammatory response, however the literature lacks information about its activity in renal tissue. Renal diseases are considered important public health problems with high potential to lead to end-stage renal disease and often to death. Focal and segmental glomerulosclerosis (FSGS) is one of the most important causes of end stage chronic renal disease. FSGS is a glomerular disease characterized by foot effacement of visceral epithelial cells (podocytes) and reducing the slit diaphragm-related protein, an important and selective structure involved on filtration barrier. These alterations lead to proteinuria and development of histological findings as vascular collapse, mesangial sclerosis and progressive deterioration of some segments of some glomeruli. Therefore, this study aims to evaluate the antiinflammatory and antifibrotic potential of butyrate in an experimental model of FSGS. Experimental FSGS mimics the classic signs of human disease and we used a single intravenous injection of chemotherapic adriamycin to induce FSGS in Balbc mice. After, the animals were treated with doses of butyrate with objective to evaluate its antiinflammatory and renoprotective properties. The treatment with butyrate in adriamycin-induced FSGS in mice reduces dramatically total urine proteins, including albumin, a marker of glomerular lesion, in addition to decreased expression of classic inflammatory markers as TGF- $\beta$ e TNF- $\alpha$. Expression of podocytes markers, such as podocin and nephrin, once reduced in disease, were re-established after butyrate treatment. Histological find- 
ings showed glomerular damage was attenuated and preserved form of the glomeruli in butyrate-treated animals when compared with controls. Here, we demonstrate that butyrate has an important effect on glomerulopathies, thus it could be a new accessible approach to treat this kind of disease.

Financial support: FAPESP CAPES (2012/02270-2).

\section{P189 \\ Perinatal Protein Restriction Alters in the Long Term the Metabolic Plasticity of Skeletal Muscle}

Raquel da Silva Aragão, Omar Guzmán-Quevedo, Georgina Perez-Garcia, Raul Manhães-De-Castro, Francisco Bolaños-Jimenez

UFPE and Nantes University

Background: Skeletal muscle has a remarkable ability to adapt its metabolism in response to caloric intake and the energy demands of the body. Thus, in response to fasting or exercise, skeletal muscle favors the use of lipids as an energy source at the expense of glucose oxidation. This metabolic adaptation, when combined with impaired mitochondrial function, leads to the development of obesity and type 2 diabetes and is associated with enhanced expression of genes involved in lipid metabolism, oxidative phosphorylation and mitochondrial biogenesis. On the other hand, it is now well established that a poor nutrient environment in utero or during neonatal life, is a predisposing factor for the development of obesity and related metabolic diseases at adulthood.

Objectives: The aim of this study was to determine whether the enhanced disease susceptibility induced by perinatal undernutrition is associated with impaired metabolic plasticity of skeletal muscle.

Methods: Wistar rats were fed isocaloric diets containing either $17 \%$ (control) or $8 \%$ protein (LP) throughout pregnancy and lactation. At weaning offspring received standard chow and at 4 months of age the effects of fasting (48h) on the expression levels in skeletal muscle of several genes involved in fatty acid metabolism and mitochondrial function were assessed by real-time PCR.

Results: In the fed state, LP rats showed enhanced expression levels of key regulatory genes of mitochondrial function and fatty acid metabolism but these effects differed from one muscle type to another. Thus, whereas the expression of CPT-1a, PGC-1 $\alpha$, UCP3 and PPAR $\alpha$ were increased in the soleus of LP rats in relation to controls, only the mRNA levels of this latter gene were increased in EDL. In contrast, we detected higher expression levels of PPAR $\delta$ in EDL but not in soleus. LP animals exhibited also impaired transcriptional responses to acute food restriction but again these responses differed between muscle types. Actually, early protein restriction suppressed the fasting-induced expression of CPT-1a in soleus but not in EDL.

Conclusions: Perinatal protein restriction alters in the long term the metabolic plasticity of skeletal muscle. This impaired capacity of muscle cells to adapt their metabolism in response to an energy challenge might contribute to the development of metabolic disorders associated with malnutrition during early life.

Financial support: CAPES-COFECUB (657/09).

\section{P190 \\ Energy Value for Overweight or Obese Children and Adolescents: a Systematic Review}

\author{
Raquel Sylvestre Ribeiro, Lúcia Gomes Rodrigues
}

Universidade Federal do Estado do Rio De Janeiro

The prevalence of childhood obesity has increased in recent decades and has now turned into a public health problem. The strategies for its treatment are poorly documented, comparing with the existing work on adults, and there are doubts in calculating diet energy value of overweight or obese children and adolescents. To describe the energy intake established in nutritional intervention studies with overweight or obese children and/or adolescents. A systematic review performed by the electronic search in the databases LILACS, IBECS, MEDLINE, Cochrane Library, SciELO. The inclusion criteria were: clinical trial study design, dietary intervention combined or not with physical activity, the target population of overweight/obesity children and/or adolescents and energy value quantitative changes in participants' diet. There were no year, genre or language specifications. The exclusion criteria were: review studies, animal experimental studies, studies with adults or old people, individuals with any syndrome or metabolic disease or in use of any medication that could influence feeding, weight and/or body composition. The review afforded 315 articles, 16 being selected to be read, 9 of whom matched the aforementioned selection criteria. All of them were published between 2004 to 2010, representing four geographic regions: Asia (44.4\%), Europe (33.3\%), North America (11.1\%) and South America (11.1\%). Three studies (33.3\%) underwent interventions with low-calorie diet, one $(11.1 \%)$ used normocaloric diet and five $(55.5 \%)$ did not classify the diet offered, only mentioning your calorie and/or distribution of macronutrients. The energy value established in the studies ranged from 1200 to $2000 \mathrm{Kcal}$. For calculating the energy needs for this population, the analyzed studies did not make clear how it was done. There was heterogeneity in the establishment of energy intake in the analyzed studies. There was no explanation from the authors of the formula for calculating energy requirements for the population in question.

\section{P191 \\ Lycopene Supplementation Modulates Leptin and Resitin in Diet-Induced Obese Rats}

Renata A.M. Luvizotto, André F. Nascimento,

Damiana T. Pierine, Erika Imaizumi, Natália C.M. Miranda, Paula T. Presti, Célia R. Nogueira, Camila R. Correa,

Kyung Jin Yeum, Ana Lúcia Anjos Ferreira

UNESP/TUFTS University

Background: Obesity is characterized by a chronic low-grade inflammation and lycopene has been reported to display anti-inflammatory effects. However, it is not clear whether lycopene supplementation modulates adipokines levels in vivo under obesity condition. 
Objectives: To evaluate whether lycopene supplementation can regulate leptin and resistin expression in obese rats.

Methods: Male Wistar rats were randomly separated for receiving control diet $(\mathrm{C}, \mathrm{n}=7$ ) or hypercaloric diet (DIO) for 6 weeks. After this period, DIO animals were randomized into two groups: DIO $(n=7)$ or DIO supplemented with lycopene (DIO + L, $n=7)$. The lycopene used was oil-resin mixed with corn oil (10 $\mathrm{mg} / \mathrm{Kg}$ body weight/day), untreated groups (C and DIO) received appropriate volumes of corn oil, both via gavage, for 6 weeks. The rats were killed by decapitation and blood samples and adipose tissue were collected for hormonal determinations and gene expression evaluation. For data analysis was used one-way ANOVA followed by Tukey post hoc tests. $\mathrm{P}<0.05$ was considered to be statistically significant.

Results: Plasma lycopene concentration was undetectable in $\mathrm{C}$ and DIO groups, while in supplemented animals it was able to be detected $(165 \pm 64 \mathrm{nmol} / \mathrm{L})$. Lycopene supplementation did not affect body weight or adiposity, however decreased leptin (DIO: $1.31 \pm$ $0.12 v s$ DIO + L: $0.97 \pm 0.22, \mathrm{p}<0.05$ ) and resistin (DIO: $1.04 \pm$ $0.17 v s$ DIO + L: $0.56 \pm 0.07, \mathrm{p}<0.05)$ gene expression and plasma levels (leptin-DIO: $10.2 \pm 2.2 \mathrm{ng} / \mathrm{mL} v s$ DIO + L: $7.1 \pm 1.3 \mathrm{ng} /$ $\mathrm{mL}, \mathrm{p}<0.05$, resistin-DIO: $10.3 \pm 2.1 \mathrm{ng} / \mathrm{mL}$ vs DIO $+\mathrm{L}: 7.7 \pm 1.3$ $\mathrm{ng} / \mathrm{mL}, \mathrm{p}<0.05$ ) in vivo, also reduced gene expression of IL-6 (DIO: $3.00 \pm 0.40 v s$ DIO + L: $0.70 \pm 0.18, \mathrm{p}<0.05)$ and monocyte chemoattractant protein-1 (MCP-1) (DIO: $1.95 \pm 0.35$ vs DIO + L: 1.01 $\pm 0.31, \mathrm{p}<0.05)$ in adipose tissue from obese rats.

Conclusions: These data suggest that lycopene affects adipokines expression and release, improving inflammatory markers, which may be indicated as an effective strategy to reduce the risk of progression of obesity-associated comorbidities.

Financial support: FAPESP (2011/22786-0).

P192

\section{Association of Bioactive Compounds of Spices with the Viability of MCF-7 Breast Cancer Cell Line}

\section{Renata Madureira Polinati, Christiane Queiroz, Marcelo Chaves Azevedo, Heloise Sierra Cordeiro, Jerson Lima Silva, Eliane Fialho}

\section{Universidade Federal do Rio De Janeiro}

Background: Spices have been used for thousands years to improve the sensory quality of foods and the quantity and variety consumed in tropical countries is particularly extensive. Among spices, black pepper is the main spice exported worldwide from India, followed by red pepper, turmeric, ginger, cardamom, curry powder, among others. These spices have BCs, such as piperine, capsaicin, curcumin and 6-gingerol which are responsible for their antioxidant and anti-inflammatory effects, contributing to their chemopreventive and chemotherapeutic activities. Breast cancer is the second most frequent type in the world and the most common among women.

Objectives: The aim of this study was to investigate the effect of piperine, capsaicin, curcumin and 6-gingerol as well as the melphalan chemotherapy, either alone or associated with the viability of the breast cancer cells (MCF-7).
Methods: For analysis of cell viability (CV), cells were treated with concentrations ranging from 1 to $300 \mu \mathrm{M}$ of BCs. For treatment with melphalan doses were $50 \%$ lower. After treatment, cells were incubated with $0.5 \mathrm{mg} / \mathrm{ml}$ MTT dissolved in PBS for 3 hours at $37^{\circ} \mathrm{C}$. After incubation, MTT solution was removed and the formazan crystals were dissolved in isopropanol acid $(0.04 \mathrm{M})$. The amount that MTT reduced was calculated based on the difference between the absorbance of 570 and $650 \mathrm{~nm}$.

Results: Due to the large volume of data, we chose the concentration of $50 \mu \mathrm{M}$ in 24 hours of treatment for the presentation of results. When it comes to the effects of the isolated compounds, we observed that curcumin decreased the $\mathrm{CV}$ in $57 \%$, followed by piperine, capsaicin, and gingerol. In the $25 \mu \mathrm{M}$ concentration, melphalan showed a decrease of $26 \%$ on $\mathrm{CV}$. After the association of BCs with melphalan we observed that for almost all combinations there was an additive effect instead of a potentiating effect, except for the combination with melphalan + piperine and melphalan + capsaicin. For the BCs combinations only an additive effect was observed rather than potentiator and for a few combinations such as piperine + gingerol the effect was less if compared to isolated use.

Conclusions: The results suggest that the combination of $\mathrm{BCs}$, piperine and capsaicin, and melphalan chemotherapy can potentiate the cytotoxic effects on MCF-7 breast cancer cells rather than when they are used alone.

\section{P193 \\ Genetic Ancestry Is Associated with Systolic Blood Pressure and Glucose in Children and Adolescents from an Admixed Brazilian Population}

Erica M. Queiroz, Ana Paula C. Cândido, Ana Maria Santos, leso M. Castro, George L.L. Machado-Coelho, Tailce M. Leite, Rinaldo W. Pereira, Renata N. Freitas

Federal University of Ouro Preto

Background: Epidemiological studies in admixed population have shown that the prevalence of obesity and related diseases such as type 2 diabetes and hypertension may vary by ethnic group, probably due to differences in the genetic Background: Risk factors for the above referred conditions are consistently found in pediatric populations and may persist until adult life.

Objectives: The aim of this study was to assess the relationship between genetic ancestry and risk variables in children and adolescents of an admixed Brazilian population.

Methods: Genetic ancestry was determined by genotyping 15 ancestry informative markers (AIMs) in 189 children and adolescents from the Ouro Preto, Brazil from which we also obtained data on anthropometric, clinical and biochemical variables. Individuals were categorized into three groups (predominantly African - PAFR, predominantly Multiethnic - PMIS and predominantly European PEUR) using the proportion of AIMs $>0.650$ as the cutoff point. Mean values of anthropometric, clinical and biochemical variables were compared between groups. Simple linear regression model was used to test differences in mean values of variables adjusting for confounders. 
Results: The contribution of European (EUR), African (AFR) and Amerindian ancestries in the population accounted for $50.3 \%$, $33.3 \%$ and $16.4 \%$, respectively. PAFR individuals had higher mean blood pressure and glucose level compared to PEUR. In the linear regression model, the difference in systolic blood pressure between the groups was independent of sex, age, body mass index, waist circumference, glucose and serum lipids. Otherwise, the difference in glucose concentrations was independent of the same variables and also of blood pressure.

Conclusions: Systolic blood pressure and glucose are positively and independently associated with a higher contribution of African ancestry in this admixed Brazilian population.

Financial support: FAPEMIG (CDS-APQ00519-09), CAPES, CNPQ (474965/2004-0), UFOP.

\section{P194 \\ Variants in the APM1, IGF2, LEPR, POMC and PPARGC1 Genes Are Associated with Components of Metabolic Syndrome in Brazilian Children and Adolescents}

Erica M. Queiroz, Ana Paula C. Cândido, leso M. Castro, Alínia Q.A. Bastos, George L.L. Machado-Coelho,

Renata N. Freitas

NUPEB/Federal University of Ouro Preto

Background: Obesity can be associated with phenotypes of the metabolic syndrome since early ages. It is well known that these phenotypes can result of the interaction between genetic and environmental factors. Several single nucleotide polymorphisms (SNPs) have been related to a higher susceptibility for metabolic syndrome phenotypes.

Objectives: To investigate whether 12 SNPs located within ADRB2, APM1, IGF2, KCNJ11, LEPR, POMC, PPARG, PPARGC1, $S L C 6 A 14$ and $U C P 2$ genes are associated with obesity or obesityrelated phenotypes including metabolic syndrome components in children and adolescents from Ouro Preto, MG.

Methods: A case-control study was conducted nested to a sectional population based study in which 192 individuals ( 7 to 14 years old) were categorized as overweight (body fat percentage $\geq 29.56 \%$ and $\geq 32.87 \%$ for boys and girls, respectively) and 211 as normal weight. Genotyping and data on clinical and biochemical variables were obtained. Carriers of the less frequent alleles for each SNP were grouped for the statistical analysis. Individuals were categorized according to cut offs of variables used to characterize metabolic syndrome in children and adolescents. Odds ratios and 95\% confident intervals for metabolic syndrome phenotypes were calculated for the polymorphic allele carriers with the most frequent allele homozigous groups as reference. For all analyses $\mathrm{P}<0.05$ was considered.

Results: No difference in the SNPs distribution was observed between normal and overweight groups. Binary logistic regression revealed that the carriers of the less frequent alleles for APM1 rs266729, POMC rs28932472 and LEPR rs1137101 had higher odds for presenting high concentrations of triacylglycerides $(\mathrm{OR}=1.65$, $\mathrm{P}=0.05)$ and LDL-C $(\mathrm{OR}=2.85, \mathrm{P}<0.01)$ and low concentrations of HDL-C $(\mathrm{OR}=2.83, \mathrm{P}=0.02)$, respectively. The POMC rs 28932472 was also marginally associated with low HDL-C $(\mathrm{OR}=2.24, \mathrm{P}=$ $0.05)$ and the IGF2 rs680 had reduced odds for high LDL-C $(\mathrm{OR}=$ $0.55, \mathrm{P}=0.02)$. The LEPR rs1137101 $(\mathrm{OR}=2.50, \mathrm{P}=0.02)$ and PPARGC1 rs8192678 $(\mathrm{OR}=1.91, \mathrm{P}=0.02)$ were associated with higher B BMI and LEPR rs1137101 was associated with high waist circumference $(\mathrm{OR}=3.46, \mathrm{P}=0.01)$.

Conclusions: We found associations between four SNPs with at least one risk phenotype that is used to characterize metabolic syndrome in children and adolescents, which might cause later in life, higher risk for cardiovascular diseases or type 2 diabetes.

Financial support: FAPEMIG, CAPES, CNPq and UFOP.

\section{P195 \\ Association Between Adiponectin Variant (T45G) and DNA Damage in Morbid Obese Women}

\author{
Bruno Cesar Ottoboni Luperini, Danielle Cristina Almeida, \\ Marília de Paula Porto, Renato Paschoal Prado, \\ João Paulo de Castro Marcondes, \\ Patrícia Fátima Souza Novais, Irineu Rasera Junior, \\ Daisy Maria Fávero Salvadori \\ Botucatu Medical School, UNESP
}

Background: Obesity is a major health problem that increases the risk of several common diseases such as increased risk of type 2 diabetes mellitus, stroke, heart disease, hypertension, metabolic syndrome, and cancer. Some studies have reported that obesity can lead to the production of oxygen reactive species, which could interact with DNA causing genotoxic damage. It is known that various hormones and cytokines are secreted by adipose tissue, among them, adiponectin. This is an important adipokine secreted by adipocytes and plays an important role in glucose regulating homeostasis. Moreover, its plasma concentration is inversely proportional to the mass of adipose tissue and is closely related to the development of metabolic syndrome (MS) and insulin resistance. Despite adiponectin's presumed role in fatty acid oxidation and energy homeostasis, little is known about the effect gene variants on DNA damage.

Objectives: Considering that, we examined the effects of adiponectin genetic variation and its relationship with DNA damage.

Methods: The ADIPOQ 45T-G single-nucleotide polymorphism (SNP) was analyzed in 300 morbid obese women living in the Southeast region of Brazil, and most of them were Caucasian. These obese women were distributed into three groups according to the genotypes: TT $(n=226$, age $33.53 \pm 6.43$ years $)$, TG $(n=68$, age $33.06 \pm$ 7.07 years) and GG $(n=6$, age $33.2 \pm 2.86$ years $)$. The control group consisted of 300 age-matched healthy Caucasian individuals with the following ADIPOQ 45T-G genotypes: TT $(\mathrm{n}=208$, age $31 \pm 4.61$ years), TG ( $\mathrm{n}=86$, age $30 \pm 6.98$ years $)$ and $\mathrm{GG}(\mathrm{n}=9$, age $31.67 \pm$ 6.95 years). DNA damage, as depicted by the comet assay, was evaluated from a maximum of 30 individuals randomly selected from each genotype, both from control and obese women.

Results: Overall, we did not observe significant differences in genotype or allele frequencies between obese and controls. Furthermore, ADIPOQ 45T-G SNP do not influence body mass index and weight in both groups. However, in obese $(37.44 \pm 32.12, \mathrm{n}=30)$ 
and in control $(17.76 \pm 22.15, \mathrm{n}=30)$, individuals with the $\mathrm{TG}+\mathrm{GG}$ genotypes had higher DNA lesions than those with TT genotype.

Conclusions: Our data demonstrated an increased DNA damage associated to the ADIPOQ 45T-G TG + GG genotypes. Nevertheless, the higher amount of DNA damage would not be related to morbid obesity, since in the control group increased genotoxicity was also detected in individuals with same genotypes.
Conclusions: Polymorphisms of genes of metabolism, DNA repair and metabolism of folic acid could modulate levels of DNA damage and efficiency of DNA repair, especially in subjects with lower intake of micronutrients. In addition, diet rich in fruit and vegetables, rich in $\beta$-carotene and lycopene, can be capable of protecting cells against DNA damage.

Financial support: FAPESP (05/54450-0).

\section{P196 \\ Influence of B-Carotene, Lycopene and Polymorphisms of the GENES GSTM1, GSTT1, GSTP1, CYP1A1, CYP2E1, XRCC1, TS and MTHFR on the Level of DNA Damage, Misincorporated Uracil and DNA Repair Capability in Individuals with Antagonic Diets}

Renato Paschoal Prado, Bruna Fornazari Santos, Daisy Maria Favero Salvadori,

Marcelo Sady Plácido Ladeira

\section{Botucatu Medical School-UNESP}

Background: The contribution of diet to cancer can vary from $10 \%$ to $80 \%$. Possibly, due to the low ingestion of antioxidants and enzymatic cofactors involved in reactions of DNA repair and methylation and the high ingestion of chemical addictive presented in the modern diet, which associated to the genetic factors, could lead to genomic instability and hipomethylation contributing to cancer development.

Objectives: We evaluated the influence of $\beta$-carotene, lycopene and polymorphisms of the genes GSTM1, GSTT1, GSTP1, CYP1A1, CYP2E1, XRCC1, TS and MTHFR on the level of oxidative DNA damage and uracil incorporation, efficiency of DNA repair in lymphocytes of two groups of individuals with different diets.

Methods: Group I (GI): 49 individuals with diet rich in organic products, integral grains, fruit and vegetables and poor in industrialized products, Group II (GII): 56 individuals with diet rich in industrialized products and poor in fruit and vegetables. Oxidative DNA damage, uracil incorporation and the efficiency of DNA repair were assessed by the Comet assay and polymorphisms by PCR or PCRRFLP. The micronutrients were measured by high-performance liquid chromatography (HPLC).

Results: The serum levels of all micronutrients were higher in individuals of GI than GII. GII presented a negative correlation between strand breaks (SBs) and $\beta$-carotene and between misincorporated uracil and $\beta$-carotene. The serum concentration of lycopene was correlated negatively with misincorporation uracil in both groups. With regard to individuals of GI: a) carriers of the MTHFR C1298C genotype showed higher levels of DNA damage and b) carriers TS $T S E R 3 R / 3 R$ genotype had higher levels of oxidized purines. With respect to individuals of the GII: a) carriers of the GSTT1 (-/-) or CYP2E1 C1/C2 or XRCC1 Arg399Arg genotype showed higher levels of oxidized purines and pyrimidines, uracil incorporated and lower efficiency of the DNA repair, b) carriers of the MTHFR C1298C genotype showed higher levels of broken DNA strand and c) genotype carriers $T S T S E R 3 R / 3 R$ had higher levels of oxidized purines and lower efficiency of DNA repair.

\section{P197 \\ Micronutrients, Total Antioxidant Capacity and Oxidative DNA Damage in Patients Without Comorbidities Undergoing Elective Surgery with Total Intravenous Anesthesia}

\author{
Mariana Gobbo Braz, Renato Paschoal Prado, \\ Leandro Gobbo Braz, Ana Lucia Dos Anjos Ferreira, \\ José Reinaldo C. Braz, Kyung-Jin Yeum, \\ Daisy Maria Fávero Salvadori \\ Botucatu Medical School and TUFTS University
}

Background: It is suggested that invasive surgical procedure can enhance reactive oxygen species (ROS) in patients. On the other hand, there are a few data in literature regarding elective surgeries, and the adjuvant effect of general anesthesia. However, it is known that propofol, an intravenous anesthetic widely used for general anesthesia, has an antioxidant potential.

Objectives: To determine the antioxidant defense and the level of oxidative DNA damage in patients without comorbidities undergoing minor surgery with intravenous propofol anesthesia.

Methods: Fifteen adult patients, of both genders, aged between 18-44 years old, classified by the American Society of Anesthesiologists as physical status I (healthy patient, with no disease other than surgical abnormality and without systemic disturbances) and scheduled for elective and minimally invasive otorhinological surgery under total intravenous anesthesia with propofol were enrolled in the study. Blood samples were drawn before (T0) and 120 min after the beginning of anesthesia (T1). Plasma was obtained to measure lipophilic antioxidants such as carotenoids, tocopherols and retinol by a high performance liquid chromatography (HPLC), hydrophilic antioxidant uric acid using a dry chemical analysis, and total antioxidant performance (TAP) by fluorometry. Oxidative DNA was evaluated in isolated lymphocytes using the alkaline comet assay.

Results: Patients presented significant decreases of carotenoids $(p \leq 0.01)$, alpha-tocopherol $(p=0.007)$, retinol $(p<0.001)$ and uric acid $(\mathrm{p}<0.001)$ concentrations, whereas significant increase of gamma-tocopherol $(\mathrm{p}<0.001)$ and TAP $(\mathrm{p}=0.015)$ was observed at T1. There was no significant change in the amount of DNA damage.

Conclusions: Despite of the reduction of endogenous antioxidants during elective surgery with propofol anesthesia, patients without comorbidities did not present significant change in the level of oxidative DNA damage in lymphocytes. Antioxidant activity of propofol and its intralipid solution containing soybean oil may be resulted in increases of plasma total antioxidant capacity and gammatocopherol concentration during anesthesia in these patients.

Financial support: Supported by FAPESP/Brazil and USDA/ USA (2010/05611-0 AND 58-1950-7-07). 


\section{P198 \\ Influence of Protein Malnutrition on Diferentiation of Bone Marrow Multipotent Mesenchymal Stem Cells}

Ricardo A. Fock, Mayara C.R. Cunha, Stela Penalva, Fabiana Lima, Primavera Borelli

Faculdade de Ciências Farmacêuticas USP

It is estimated that over $30 \%$ of humanity will suffer from some degree of malnutrition was found more in children, the elderly, patients with malignancies, chronic diseases or undergoing chemotherapy. Data showed that protein malnutrition (PM) compromises lympho-hematopoietic organs and modifies the immune response. Knowing this and the bone marrow (BM) microenvironment has distinct modulators that act synergistically to influence hematopoietic tissue, we evaluated some aspects of the complex regulation of BM microenvironment evaluating the expression of transcription factors involved in the differentiation of MSC, and the production capacity of cytokines which are important in the regulation of the hematopoiesis. Two-month-old male BALB/c mice were submitted to PM with a low-protein diet $(2 \%)$, compared to $12 \%$ protein in the control group. Serum total protein levels and albumin as well as complete blood count and BM aspirate smear were evaluated. MSC from BM were isolated, characterized and the expression of PPAR $\gamma$, RUNX2 were evaluated. Supernatants of the MSC culture were collected and the production of Stem cell factor (SCF), granulocyte colony stimulating factor (G-CSF) and granulocyte/macrophage colony stimulating factor (GM- CSF) were quantified. The malnourished groups reduced levels of total serum protein and albumin associated with anemia, leukopenia and hypoplasia of BM. MSC characterized by flow cytometry showing cells populations in both groups stained positive for CD90, CD271, Sca1, CD49e, CD13, low marking for CD34 and negative for CD14, CD45. In the measurement of transcription factors by Western Blot found that MSC of malnourished animals showed higher expression of RUNX2 $(0.71 \pm 0.08,0.49 \pm, 0.07 \mathrm{UA}$ control group, $\mathrm{p} \leq 0.05)$ and PPAR $\gamma(0.67 \pm 0.05 \mathrm{UA}, 0.51 \pm 0.07 \mathrm{UA}$ control group, $\mathrm{p} \leq 0.05)$. MSC supernatants of malnourished mice quantified by Elisa presented a higher concentration of SCF (144 \pm 7 $\mathrm{pg} / \mathrm{mL}$.day, $\mathrm{p} \leq 0.05)$ and a reduced concentration of G-CSF $(42 \pm 19$ $\mathrm{pg} / \mathrm{mL}$.day, $\mathrm{p} \leq 0.05)$ and GM-CSF $(2,2 \pm 0.8 \mathrm{pg} / \mathrm{mL}$.day, $\mathrm{p} \leq 0.05)$ when compared to the control group $(119.1 \pm 4.9 \mathrm{pg} / \mathrm{mL}$.dia, $210.0 \pm$ $94 \mathrm{pg} / \mathrm{mL}$.dia and $12.9 \pm 3.4 \mathrm{pg} / \mathrm{mL}$.dia, respectively). The alterations allow us to conclude that malnutrition commits MSC contributing to an impaired hematopoietic microenvironment and inducing the bone marrow failure observed in PM states.

Financial support: FAPESP.

\section{P199 \\ STAT1 and STAT3 Expression in Spleen Lymphocytes from a Protein Malnourished Mice}

\begin{abstract}
Alexandra Siqueira Mello, Dalila Cunha Oliveira, Jackeline Soares O. Beltran, Stela Penalva,

Primavera Borelli, Ricardo Ambrósio Fock
\end{abstract}

Department of Clinical and Toxicological Analyses, Faculty of Pharmaceutical Sciences, University of São Paulo

Protein malnutrition (PM) modifies resistence to infection altering a wide range of physiological processes, including hematopoiesis and immune response. The STATs are transcription factors involved in signaling pathways and has been implicated in a variety of cellular immune functions like synthesis of different cytokines and growth factors which are important for the immune response. In this study, we evaluated the effect of PM on the Lymphocytes Stat1 and Stat3 expression. Two-month-old male BALB/c mice were submitted to PM. When the experimental group lost approximately $20 \%$ of their initial body weight, hemogram, myelogram and spleenogram were evaluated. The spleen cells populations were analyzed by flow cytometry and the STAT1 and 3 were quantified. PM group presented anemia, leucopenia and reduction in total spleen cells cellularity. PM group showed a higher percentage of spleen CD4 positive cells (75.5 $\pm 6.8 \%)$ and more CD3 positive spleen cells $(28.5 \pm 2.3 \%)$ when compared to control animals $(65.0 \pm 7.2 \%$ and $17.8 \pm 5.0 \%, \mathrm{p} \leq 0.05)$. STAT1 and STAT3 expression did not show difference when compared control to PM group. However cells from PM group stimulated with LPS showed higher expression of STAT $1(0.8500 \pm 0.1500)$ and lower expression of STAT3 $(0.2725 \pm 0.03065)$ when compared to control group $(0.2067 \pm 0.1468,0.8575 \pm 0.1425, \mathrm{p} \leq 0.05)$. These findings show that malnourished mice present a deficient immune response when challenged.

Financial support: FAPESP (2010/51921-0).

P200

The Influence of Protein Malnutrition on the Expression of TNFRI Receptors and IL-1ß Production in Macrophages Stimulated with TNF- $\alpha$

Dalila Cunha Oliveira, Alexandra Siqueira Mello, Jackeline Soares Oliveira Beltra, Ed Wilson Santos, Primavera Borelli, Ricardo Ambrósio Fock

Department of Clinical and Toxicological Analyses, Faculty of Pharmaceutical Sciences, University of São Paulo

Protein malnutrition (PM) decreases resistance to infection, previous studies have demonstrated in malnourished animals a decreased capacity of pro-inflammatory cytokines production. TNF- $\alpha$ is a pro inflammatory cytokine primarily produced by monocytes and macrophages and it is able to initiate inflammatory and immunoloregulatory responses by binding to TNF cell surface receptors inducing $\mathrm{NF}-\kappa \mathrm{B}$ activation and transcription of pro-inflammatory cytokines. 
Our work purpose to investigate the capacity of macrophages have to produce IL- $1 \beta$ after TNF- $\alpha$ stimulus and the expression of TNF receptor type I (TNFRI) in a murine model of PM. Two-month-old male BALB/c mice were submitted to PM with a low-protein diet ( $2 \%$ ), compared to $12 \%$ in the control group. Serum total protein levels, albumin, hemogram and peritoneal cellularity count were evaluated. Macrophages from both groups were stained with F4/80, Mac-1 and CD14 and stimulated with $10 \mathrm{pg} / \mathrm{mL}$ of TNF- $\alpha$ and after 2 hours of stimulus the IL- $1 \beta$ production and expressions of TNFRI were quantified. Malnourished animals presented reduced serum total protein $(5.0 \pm 0.24 \mathrm{~g} / \mathrm{dL}, \mathrm{p} \leq 0.05)$ and albumin $(1.8 \pm 0.14 \mathrm{~g} / \mathrm{dL}, \mathrm{p} \leq 0.05)$ levels as well as anemia $(10.1 \pm 1.1 \mathrm{Hb} . \mathrm{g} / \mathrm{dL}, \mathrm{p} \leq 0.05)$, leucopenia $(1050 \pm 170$ leuc. $/ \mathrm{mm} 3, \mathrm{p} \leq 0.05)$ and reduction in peritoneal cellularity $(1.8 \pm 0.6 \times 106 \mathrm{cel} / \mathrm{mL}, \mathrm{p} \leq 0.05)$ when compared to control animals $(6.551 \pm 0.2813$ prot. g/dL, $2.811 \pm 0.1327$ alb. g/dL, $13.2 \pm 1.4 \mathrm{Hb} . \mathrm{g} /$ $\mathrm{dL}, 3240 \pm 320$ leuc. $/ \mathrm{mm}^{3}, 2.7 \pm 0.7 \times 10^{6} \mathrm{cel} / \mathrm{mL}$, respectively). Macrophages from malnourished group after TNF- $\alpha$ stimulus showed a reduced production of $\mathrm{IL}-1 \beta(39.5 \pm 21.8 \mathrm{pg} / \mathrm{mL}, \mathrm{p} \leq 0.05)$ when compared to animals from controls groups stimulated (116.8 \pm $29.8 \mathrm{pg} / \mathrm{mL})$ or not with TNF- $\alpha(122.0 \pm 22.7 \mathrm{pg} / \mathrm{mL})$. The quantification of F4/80, Mac-1 did not show difference beteween groups, however macrophages from malnourished animals showed a reduced expression of CD14 $(50.1 \pm 2.5 \%)$ when compared to control animals $(65.1 \pm 3.2 \%, \mathrm{p} \leq 0.05)$. The expression of TNFRI showed reduced values in malnourished group without $(0.36 \pm 0.12 \mathrm{U} / \mathrm{A}, \mathrm{p} \leq 0.05)$ or with $(0.36 \pm 0.06 \mathrm{U} / \mathrm{A}, \mathrm{p} \leq 0.05) \mathrm{TNF}-\alpha$ stimulus when compared to the respective control animals $(0.80 \pm 0.09 \mathrm{U} / \mathrm{A}$ and $0.76 \pm 0.15 \mathrm{U} / \mathrm{A})$. Our results show that cells from malnourished animals have reduced expression of receptors and low cytokine production these results infer us to say that these reduced receptors expression could be one of the cause that malnourished individuals would not respond with the same intensity and/or manner as well-nourished individuals.

Financial support: FAPESP (2011/14247-2).

\section{P201 \\ Docosahexaenoic Acid (DHA) Induces Epigenetic Changes Involved in the Transcriptional Activation of Tumor Suppressor Gene in Breast Cancer Cell Line}

Rita de Cássia Borges Castro, Aline Conti, Danielle Fontes Almeida, Fernando Salvador Moreno,

Thomas Prates Ong, Graziela Rosa Ravacci, Dan Linetzky Waitzberg

Faculdade de Medicina da Universidade De São Paulo

Background: DNA hypermethylation and alterations in posttranslational histones modifications contribute to silencing of tumorsuppressor genes. These alterations are commonly seen in human cancers and have an important role in mammary tumorigenesis. Several genes are hypermethylated in breast cancer, especially the tumor suppressor gene $R A S S F 1 A$, which contribute to its reduced expression in breast tumors. Epigenetic events are important as therapeutic targets, because of its reversible nature after dietary compounds that can modulate the epigenetic processes in cancer. Experimentally, docosahexaenoic acid (DHA), a member of the omega-3 fatty acids, can reduce proliferation, induce apoptosis and decrease the invasive potential of breast tumor cells. However, the antitumor mechanism of DHA and its ability to modulate epigenetic events are not completely understood.

Objectives: Our objective was to verify, in vitro, the action of DHA in epigenetic events related to transcriptional reactivation of tumor suppressor gene, such as $R A S S F 1 A$, in different human breast cancer cell lines.

Methods: Three different breast cell lines (MCF-7, MDA-MB231, SKBR-3) were treated with DHA $(100 \mu \mathrm{M})$ or vehicle (ethanol) for 72 hours. Real time quantitative PCR was performed for gene expression quantification of RASSF1A. DNA methylation of the promoter region of $R A S S F 1 A$ was evaluated by methylation specific PCR. Western blot was used to analyze histone modification, as histone H3 lysine 9 (H3K9ac) and histone H4 lysine 16 (H4K16ac) acetylation. Moreover, we evaluated the phases of the cell cycle by flow cytometry.

Results: Compared to control cells, DHA increased RASSF $1 A$ expression on MCF-7 (1,98 fold, $\mathrm{p}=0.03$ ), but not in MDA-MB231 and in SKBR-3 cells. These three transformed breast cells lines show methylation in specific region of RASSF1A promoter. DHA treatment increased $R A S S F 1 A$ promoter region hypomethylation in MCF-7 and SKBR3. Moreover, DHA induced H4K16ac in MCF7 $(\mathrm{p}=0.04)$ and MDA-MB231 $(\mathrm{p}=0,03)$, demonstrating a role in facilitating gene transcription.

Conclusions: From this perspective, we can suggest that DHA could act by epigenetic mechanisms related to transcriptional reactivation and reactivate the tumor gene supressor, as $R A S S F 1 A$, previously silenced.

Financial support: FAPESP (2010/19706-2).

\section{P202 \\ Açai (Euterpe Oleracea Mart.) Extract Induces the Expression of Glutathione S-Transferase and Protects Caenorhabditis Elegans Against Oxidative Stress Conditions}

\author{
Larissa F. Bonomo, Alvaro G.J. Torres, Patricia Boasquivis, \\ Joyce F.C. Guerra, Maria L. Pedrosa, Marcelo E. Silva, \\ Riva P. Oliveira \\ Universidade Federal de Ouro Preto
}

Açai (Euterpe oleracea Mart.) is a native palm plant in northern South America which has recently emerged as a promising source of natural antioxidants, mainly polyphenols. The pulp from the açai fruit has received much attention in recent years as one of the new "super fruits" due to its high antioxidant capacity and potential anti-inflammatory activities. In this work the Caenorhabditis elegans was used as a model organism to investigate açai aqueous extract (AAE) effects on oxidative stress resistance and its possible mechanisms of action. We determined the AAE capacity to inhibit DPPH radical as indicative of its capacity to neutralize free radicals. We observed that 100 $\mathrm{mg} / \mathrm{mL}$ of AAE inhibited $72,16 \%$ of DPPH radicals and this capacity was not significantly altered when the extract was mixed with Escherichia coli, the food source for C. elegans. Next, we examined whether AAE induced the expression of gst-4, a glutathione 
S-transferase enzyme, using a transgenic line carrying the gst-4 promoter fused to the Green Fluorescent Protein (GFP) gene. The fluorescence signal of gst $-4: \because G F P$ animals treated with $100 \mathrm{mg} / \mathrm{mL}$ of AAE for 48 hours were significantly increased compared to control group. This same treatment was neither able to change catalase activity nor to increase the levels of total protein sulfhydryl groups in wild-type animals. However, when the worms were first exposed to AAE and then submitted to stress condition with t-butyl hydroperoxide (t-booh), this pretreatment was able to prevent the reduction of protein sulfhydryl groups level's induced by t-booh. In addition, AAE pretreatment increased significantly the animal's survival after 12 hours on t-booh. To further investigate how AAE increased the oxidative stress resistance, survival on stress conditions were performed using C. elegans deletion mutants for the $\mathrm{p} 38$ mitogen-activated protein (MAP) kinase family, a pathway which is activated by a variety of environmental stresses. By using nsy-1 and sek-1 mutants, which encodes the MAPKKK and MAPKK respectively, we observed that AAE exposure also enhanced their resistance to oxidative stress. Our data indicates that $\mathrm{p} 38 \mathrm{MAPK}$ does not seem to be required for AAEmediated stress resistance.

Financial support: FAPEMIG and CNPQ.

\section{P203 \\ Identification G1793A Mutation of the Gene Encoding the Enzyme Methylenetetrahydrofolate Reductase and Environmental Factors in Women with Breast Cancer}

Sandra Soares Melo, Mariel de Barros, Cláudia Helena Jasper, Mônica Testoni Cardozo, Bruno H. Gern

Universidade do Vale Do Itajaí

Background: The etiology of breast cancer may be influenced by genetic and environmental risk factors, being related to mutations, family history of the disease, hormonal, nutritional and behavioral issues.

Objectives: The study aimed to characterize the mutation G1793A of the gene encoding the enzyme methylenetetrahydrofolate reductase (MTHFR) and environmental factors in women with breast cancer.

Methods: The sample consisted of 35 women with breast cancer, treated at a Basic Health Unit in the city of Itajai - SC. Gene and allelic frequency were determined from polymorphism G1793A. Anthropometric data was collected and a clinical and a food frequency questionnaire were apllied. The presence of the mutation G1793A was determined through the amplification of the fragment exon 11 of the MTHFR gene by PCR-RFLP.

Results: The results showed heterozygous genotype frequency of $5.71 \%$ for the G1793A mutation. The average age of the sample was 56 years, most women were overweight, had no family history of breast cancer, past use of oral contraceptive, had gone through mammography and had breast fed, multiparity and were postmenopausal. Coffee, milk and soy bean oil were the daily most consumed foods.
Conclusions: It concludes that G1793A genotype showed a low frequency in the population studied. The environmental factors checked in the women were unfavorable socioeconomic conditions, physical inactivity, obesity, multiparity, low exclusive breastfeeding duration, high fat intake including high red meat consumption and low consumption of fruits and vegetables. Considering the low genotype frequency of the G1793A mutation, we suggest the development of case-control studies with a larger sample size, to be possible to do associations between environmental risk factors and gene mutation.

Financial support: Governo do estado sc programa pesquisa artigo 170 .

\section{P204 \\ Lipidemia and Blood Lactate Assesment in Patients with Colorectal Cancer Supplemented for 8 Weeks with Fish Oil}

Sandro J.R. Bonatto, Isabela Coelho, Daniele Pequito, Adriana Aya Yamaguchi, Gina Borghetti, Júlia Aikawa, Luciano Vale Sabóia, João Repka, Jorge Itsu Fukushima, Luiz Claudio Fernandes

Instituto de Pesquisa Pelé Pequeno Príncipe

Objective: The chemotherapeutic agents can alter the plasma lipid profile. The polyunsaturated fatty acid (PUFA) n-3 is able to minimize this situation. This study investigated whether supplementation for 8 weeks with $2 \mathrm{~g}$ / day of fish oil rich in PUFA n-3 alters the blood lipid and blood lactate concentration in plasma of cancer patients.

Methods: This experimental protocol was approved by the Ethics Committee of Angelina Caron Hospital, Campina Grande do Sul, Parana, Brazil, and was endorsed by the Committee of the Universidade Federal do Paraná. Receiving chemotherapy after excision of gastrointestinal tumors $(\mathrm{n}=38)$ were randomized, and one group was supplemented $(\mathrm{n}=19)$ with $2 \mathrm{~g}$ of fish oil (Herbarium) daily for 8 weeks and the other was not supplemented $(n=19)$. Blood samples were collected prior to initiation of supplementation (T0) and after 8 weeks (T8) in both groups. The plasma was separated by centrifugation, and is measured $(\mathrm{mg} / \mathrm{dL})$, total cholesterol, HDL, LDL, VLDL triacylglycerols by commercial systems (LABEST) and lactate $(\mathrm{mmol} / \mathrm{ml})$ by the enzymatic method. The results were expressed as mean $\pm \mathrm{SEM}$. Total cholesterol in control group T0 was $162.4 \pm 14.5$, T8 was $196.3 \pm 10.2$, in fish oil group T0S was $174.1 \pm$ 9.5 , T8S was $154.4 \pm 9.4$. Triacylglycerol T0 was $144.6 \pm 19.4$, T8 was $170.7 \pm 12.6$, T0S was $134.4 \pm 12.8$, T8S was $131 \pm 10.4$. HDL T0 was $35.3 \pm 4.7$, T8 was $33.9 \pm 5.1$, T0S was $30.6 \pm 3.1$, T8S was $44.46 \pm 3.9$. LDL T0 was $105.8 \pm 11.2$, T 8 was $127 \pm 10.2$, T0S was $109.6 \pm 8.3$, T8S was $93.5 \pm 7.1$. VLDL T0 was $29.16 \pm 4.6$, T8 was $35.8 \pm 3.1$, T0S was $26.1 \pm 2.6$, T8S was $25 \pm 1.2$. Lactatemia T0 was $1.83 \pm 0.07$, T8 was $1.74 \pm 0.1$, T0S was $1.75 \pm 0.1$, T8S was $1.37 \pm$ 0.07 .

Conclusion: Supplementation with fish oil was effective in reducing blood lactate concentration and increase HDL in relation to the start of supplementation. And also to reduce total and LDL cholesterol compared with subjects not supplemented.

Financial support: Pronex-CNPq, CAPES and Fundação Araucaria. 
P205

Effect of Fish Oil on Insulin Sensitivity and Global DNA Methylation in Rats Supplemented for Two Generations

Renata Gorjao, Sandro M. Hirabara, Alessandra Folador, Jarlei Fiamoncini, Rafael H. Lambertucci,

Carlos F. Rodrigues Jr., Marlene S. Rocha, Julia Aikawa,

Ricardo K. Yamazaki, Amanda R. Martins,

Alice C. Rodrigues, Angelo R. Carpinelli, Tania C. Pithon-

Curi, Luiz C. Fernandes, Rui Curi

ICB-USP and ICAFE-UNICSUL

Evidence suggests that omega-3 (n-3) polyunsaturated fatty acids (PUFA), mainly eicosapentaenoic (EPA) and docosahexaenoic (DHA) acids, have positive impact on health in humans and animals models, but the underling mechanisms are not completely known yet. Here, we investigated the effect of fish oil supplementation for two consecutive generations on systemic and muscle insulin sensitivity in rats associated to total DNA methylation profile in skeletal muscle and liver. Female Wistar rats were supplemented with fish oil or water prior to mating and then throughout pregnancy and gestation. Supplementation was daily and orally given at $1 \mathrm{~g}$ per kg body weight as a single bolus. Same treatment was performed by the two following generations. At 75 days of age, male offspring were used. Plasma concentrations of glucose, insulin, and lipids were determined at the end of experimental protocol. HOMA-IR, ITT, muscle insulin sensitivity (glucose uptake and metabolism, insulin signaling, and GLUT-4 translocation), and muscle lipid composition were also evaluated. Data were analysed by $t$ test or One-Way Anova followed by Tukey post-test. Long-term fish oil supplementation reduced glycemia and lipidemia and improved in vivo insulin sensitivity (HOMA-IR and ITT) as well as in vitro insulin sensitivity in soleus muscle (glucose uptake and metabolism and GLUT-4 translocation). The last effect was associated with increased insulin-stimulated p38 MAP kinase phosphorylation and lower n-6/n-3 fatty acid ratio, but not with IR, IRS-1, and Akt phosphorylation induced by insulin. Global DNA methylation was significantly decreased in liver from $\mathrm{G} 2$ rats by $50 \%$ $(p<0.05)$, but it was not modified in soleus skeletal muscle. These results suggest that long-term fish oil supplementation improves insulin sensitivity in association with increased insulin-stimulated p38 activation, decreased n-6:n-3 fatty acid ratio in skeletal muscle, and decreased global DNA methylation in liver.

Financial support: FAPESP, CNPQ, CAPES, and INOD.

\section{P206 \\ Prevention of High-Fat Diet-Induced Insulin Resistance by Fish Oil Supplementation Is Associated with Modulation of Genes Related to Muscle Mitochondrial Function and Oxidative Capacity in C57BL/6 Mice}

\author{
Amanda R. Martins, Alice C. Rodrigues, Laureane N. Masi, \\ Cátia L. Amaral, Amanda R. Crisma, Rui Curi, \\ Sandro M. Hirabara \\ ICB-USP and ICAFE-UNISCUL
}

Obesity induced by high-fat diet is closely related to the development of several diseases, including insulin resistance syndrome, type 2 diabetes mellitus, dyslipidemias, and inflammatory diseases. Although the factors leading to insulin resistance in these conditions are not completely defined yet, evidence suggests that the increased available of fatty acids (FA) plays an important role in the development of muscle insulin resistance. On the other hand, intake of polyunsaturated fatty acids (PUFA), specially n-3 PUFA, is associated with beneficial effects on insulin resistance, but the involved mechanisms remain to be clarified. In this study, we evaluated if the effects of the fish oil supplementation, a source of n-3 PUFA, on insulin resistance induced by high-fat diet in $\mathrm{C} 57 \mathrm{Bl} / 6$ male mice is associated with alteration in the expression of genes related to glucose and lipid metabolism. C57BL/6 male mice ( 8 weeks) were divided in four groups: a) control diet (CD), b) HFD, c) CD supplemented with n-3 $(\mathrm{CD}+\mathrm{n}-3)$, and d) HFD supplemented with n-3 (HFD + n-3). CD + n-3 and HFD + n-3 were supplemented with fish oil (n-3 source) by oral gavage at $2 \mathrm{~g}$ per $\mathrm{Kg}$ of body weight, three times per week. $\mathrm{CD}$ and HFD were supplemented with water instead at the same dose. Our results showed that fish oil supplementation increased insulin sensitivity, reduced weight gain and improved lipid profile in animals submitted to high-fat diet. Moreover, expression of oxidative and lipogenic genes was modified in soleus muscle from these animals. IRS-1, UCP3, PLIN5, and PGC-1a genes were significantly downregulated, GLUT4 gene was not modified, and CPT1 gene was upregulated in animals fed a high-fat diet and supplemented with fish oil. In summary, the results suggest that the prevention of skeletal muscle insulin resistance induced by high-fat diet by fish oil supplementation is associated with modulation of expression of genes related to the mitochondrial function and oxidative capacity. Acknowledgements: FAPESP, CNPq, CAPES, Dean's Office for Research/USP, INOD, NEL, and Center of Lipid Education and Research. 


\section{P207}

\section{Influence of Modifier Genes Pre-Selected in BRAC1 Carriers on Cancer Risk in Patients with CHEK2 Mutations}

\section{Satish Gupta, Katarzyna Jaworska, Katarzyna Durda, Magdalena Muszynska, Ania Jakubowska, Jan Lubinski \\ Medical University of Warsaw}

The major inherited risks of development of breast and other cancers are germline mutations in either the high (BRCA1 BRCA2) or moderate (CHEK2) risk genes. The substantial variability in cancer penetrance in mutation carriers probably can be explained by influence of other genetic or environmental factors. The knowledge of risk-modifying factors in addition to mutation status would aid in the effective application of risk prediction or cancer prevention strategies in patient carrying these mutations. In Poland the three common founder mutations in BRCA1 gene which account for the majority $(\sim$ $90 \%$ ) of BRCA1 mutations in breast-ovarian cancer families and four mutations in CHEK2 gene (1100delC, IVS2 + 1G > A, I157T, del5395) which are present in $\sim 6 \%$ of general Polish population have been identified. Studies on our population provided evidence that CHEK2 truncating mutations are associated with increased risk of cancer in the thyroid, breast and prostate. The I157T has been found to be associated with increased risk of breast, prostate, colon, kidney and thyroid cancer. Presence of founder mutations of BRCA1 and CHEK2 genes in our population makes it ideal for association studies on other genes modifying cancer risk not influenced by ethnic variations. We have chosen group of Selenoprotein coding genes for this kind of association study, as Selenium have been studied extensively in different kind of cancers with respects to its protective capabilities. We found lower Se concentration in cancer patients than in controls $(73.25 \mu \mathrm{g} / \mathrm{L}$ vs. $82.05 \mu \mathrm{g} / \mathrm{L})$. Next we divided the group according to type of cancer: breasts, prostate, lung/larynx and other cancer-control pairs. We compared the genotype frequencies for 4 most polymorphic tested SNPs (rs1139793, rs713041, rs1050450 and rs5845). We found some evidence that specific genotypes in tested genes may be associated with different cancer risk depending on Se concentration, however it has to be verified in larger number of pairs.

\section{P208 \\ Nutrigenetic Interaction Between Uncoupling Proteins and Dietary Plant Steroids Alters Energy Metabolism}

\section{Mickey Wilson, Debora Esposito, Slavko Komarnytsky \\ North Carolina State University}

Background: Metabolic syndrome is defined as the pathophysiologic condition characterized by the presence of co-existing traditional risk factors for cardiovascular disease such as obesity and insulin resistance. Lifestyle modification can improve insulin resistance and mitigate risk factors associated with the metabolic syndrome, yet the success of maintaining lifestyle changes in humans over a long period is poor. Our previous studies have shown that plant steroids attenuate lipid accumulation in the adipocytes and mitigate obesity by an unknown mechanism.

Objectives: The cellular mechanisms that contribute to metabolic syndrome are not fully understood, but evolving concepts suggest that hypothalamus, liver, and adipose tissue defects play major roles. The present study was designed to determine the potential of plant steroid supplementation to modify metabolic gene expression profiles in a mouse model of diet-induced obesity.

Methods: Three groups of $6 \mathrm{wk}$ old male C57BL/6J mice $(\mathrm{n}=$ 10) fed low fat $(10 \%)$ or high fat $(60 \%)$ diet were gavaged daily for $28 \mathrm{~d}$ with $0.2 \mathrm{ml}$ of vehicle (5\% DMSO in corn oil) or $50 \mathrm{mg} / \mathrm{kg}$ body weight of plant steroids. Body composition was assessed by dualenergy X-ray absorptiometry, while hypothalamus, liver, and adipose tissue samples were subjected to the pathway-specific real time PCR analysis.

Results: Oral administration of plant steroids decreased food intake, body weight gain, and fat mass as compared with vehicletreated controls. Expression levels of the brain-derived neutotrophic factor (Bdnf) mRNA were increased in adipose (4.5 fold), liver (4.5 fold), and hypothalamic (2 fold) tissues, suggesting enhanced norepinephrine turnover and whole body carbohydrate oxidation. Uncoupling protein 1 (Ucpl) transcript was strongly upregulated only in the adipose tissue (16 fold), consistent with alterations in lipid and fatty acid metabolism.

Conclusions: The fat tissue gene expression profile indicates a positive shift in carbohydrate and lipid metabolism associated with dietary plant steroids and begins to elucidate their putative cellular targets and mechanisms.

\section{P209 \\ Effects of Safflower Oil on Body Fat and Biochemical Parameters in Rats Fed a Hypercaloric Diet}

\author{
Elisângela M.S. Pérez, Fernanda T. Benevides, \\ Nylane M.N. Alencar, Ingrid F.T. Figueiredo, \\ Karoline S. Aragão, Otacílio B. Deocleciano, \\ Socorro V.F. Madeira \\ Universidade Federal do Ceará
}

Background: Obesity is a medical condition that increases the risk of cardiovascular disease. In some industrialized countries, the safflower oil has been used to reduce the excess of body fat. Safflower oil is a natural oil made from safflower seeds, rich in the essential n-6 polyunsaturated fatty acid (PUFA) linoleic acid.

Objectives: The aim of this work was to evaluate the effect of safflower oil on body fat and serum parameters in rats.

Methods: Male Wistar rats (90-110g) were randomly divided into three groups $(\mathrm{n}=11)$. Groups A and B were fed with a normocaloric diet and group $\mathrm{C}$ with a hypercaloric diet for 31 weeks. Groups $\mathrm{B}$ and $\mathrm{C}$ were treated with safflower oil $(40 \mathrm{mg} / \mathrm{Kg})$ by gavage, during the last seven weeks. Weekly body weights, daily water and food consumption were recorded and at the end of 31 weeks heparinized blood samples were collected for determination at serum levels of total cholesterol (TC), glucose (GLU), and triglycerides (TG) by commercial kits. Data were expressed as mean \pm standard error from mean 
(S.E.M.) adopting as significant results had presented $\mathrm{p}<0.05$ (ANOVA and Tukey post test). Experimental protocols were approved by the Institutional Ethics Committee.

Results: After 23 weeks with hypercaloric diet group $\mathrm{C}$ showed a significant $(p<0.05)$ increase in body weight equivalent to $75 \%$ and $115 \%$, when compared to animals fed on standard diet, group A and $\mathrm{B}$, respectively. The administration of safflower oil in the begin of 24 week reduced significantly the increase of weight in group B $(311.75 \pm 14.00 \mathrm{~g}$ to $319.75 \pm 12.15 \mathrm{~g}, 102.57 \%)$ compared to group A $(321.00 \pm 10.12 \mathrm{~g}$ to $346.00 \pm 9.45 \mathrm{~g}, 107.78 \%)$, but has no significantly effect in group C. No significant differences among the experimental groups were found TC and GLU. The serum levels of TG were significantly increased in group $\mathrm{C}$ with hypercaloric diet $(96.25 \pm 5.65 \mathrm{mg} / \mathrm{dL})$ compared to normocaloric diet group A (61.92 $\pm 2.78 \mathrm{mg} / \mathrm{dL})$. The safflower oil reduced significantly TG in group B $(46.05 \pm 4.45 \mathrm{mg} / \mathrm{dL})$ compared to group A, but has no significantly effect in group $\mathrm{C}$.

Conclusions: These results suggest that safflower oil reduced TG but has no significantly effect in obesity.

Financial support: CNPQ.
(109.3- 245) $\mathrm{kg}, \mathrm{BMI}=23.7 \pm 0.52 \mathrm{~kg} / \mathrm{m}^{2}$ and $51.2 \pm 1.06 \mathrm{~kg} / \mathrm{m}^{2}$, body fat $=29.9 \pm 2.0 \%$ and $49.4 \pm 0.9 \%$. The adipocytes area was $62 \%$ higher in SC of obese $(8054 \pm 351 \mu \mathrm{m} 2)$ compared to control $(4959 \pm 434 \mu \mathrm{m} 2)$ subjects. This increase was even greater $(86 \%)$ in OM of obese $\left(7126 \pm 121 \mu \mathrm{m}^{2}\right)$ compared to controls $(3835 \pm$ $543 \mu \mathrm{m}^{2}$ ). Leptin expression, characteristically produced by SC fat was similar between Leptin expression, characteristically produced by SC fat was similar between groups in that site. In the OM fat, leptin's expression was $218 \%$ higher in obese $(1.267 \pm 0.21)$ compared to control $(0.40 \pm 0.15)$ patients. GLUT4 expression was similarly reduced in SC and OM of obese $( \pm 75 \%)$ compared to control ones. IRS-1 expression did not differ between both SC, while in OM fat, it was reduced in obese subjects [0.33 (0.01- 0.69)] compared to controls $[0.82(0.57-1.04)]$. There were no differences between expression of adiponectin and its receptor 1 .

Conclusion: Despite the expansion of both visceral and subcutaneous fat, increased expression of some important marker occurs mainly in the visceral tissue, including leptin that is essentially produced in subcutaneous tissue.

Financial support: $\mathrm{CNPQ}$.
P210

\section{Class III Obesity Is Related to Alterations in Expression of Leptin, Insulin Receptor Substrate-1 (IRS-1) in the Visceral But Not in the Subcutaneous Fat}

Solange Silveira Pereira, Lilian Gonçalves Teixeira, Marina Chaves Oliveira, Alexandre Lages Savassi Rocha, Marco Túlio Costa Diniz, Adaliene V. Matos Ferreira, Jacqueline Isaura Alvarez Leite

Universidade Federal de Minas Gerais

Background: Visceral fat is more related to the production of pro-inflammatory adipokines, whereas the subcutaneous fat is linked to the production of leptin and adiponectin. It is known that expansion of visceral fat is more deleterious than the subcutaneous fat. In the extreme obesity the adipokines' profile in those tissues is not fully clarified.

Objectives: To evaluate the profile of production and expression of adipokines in visceral (omental) and subcutaneous (abdominal) fat in class III obese patients (BMI $>40 \mathrm{~kg} / \mathrm{m}^{2}$ ) compared with tissues from eutrophic ones.

Methods: Sixteen obese patients (undergoing bariatric surgery) and 14 normal ones (undergoing gynecologic or abdominal hernia surgeries) at the Hospital das Clínicas of UFMG participated of the study. At the time of surgery were collected samples of abdominal subcutaneous(SC) and omental(OM) fats. Body weight, BMI and percentage body fat (by BIA) were measured. Adipocytes area was presented as the average of 100 cells/subject. Expression of leptin, adiponectin and its receptor-1, GLUT- 4 and IRS- 1 were measured by RT-PCR. Normal distribution data were expressed as mean $\pm \mathrm{SE}$, and analyzed by $t$ test while other data were expressed as median/interquartile range and analyzed by Mann-Whitney test.

Results: The anthropometric data of eutrophic(CT) and obese $(\mathrm{OB})$ patients were: weight $=60.9(51-74.5) \mathrm{kg}$ and 131.7

\section{P211 \\ Protective Effect of Lithospermum Erythrorhizon Against Diet-Induced Obesity Through the Modulation of Hepatic Gene Expressions Involved in Lipid Metabolism}

So Young Gwon, Jiyun Ahn, Chang Hwa Jung, Taeyoul Ha

Korea Food Research Institute

Lithospermum erythrorhizon, which has traditionally been used as a vegetable and to make the liquor Jindo Hongju, contains several naphthoquinone pigments, including shikonin. This study aimed to evaluate the anti-obesity effects of Lithospermum erythrorhizon ethanol extract (LE) and elucidate the underlying mechanism. C57BL/6J mice were fed a normal or high-fat diet with or without LE supplementation for 8 weeks. LE reduced high-fat diet-induced increases in body weight, white adipose tissue mass, serum triglyceride and total cholesterol levels, and hepatic lipid levels while decreasing lipogenic and adipogenic gene expression. Furthermore, acetylshikonin suppressed adipocyte differentiation in a dose-dependent manner and significantly attenuated adipogenic transcription factor expression in 3T3-L1 cells. These findings suggest that Lithospermum erythrorhizon prevents obesity by inhibiting adipogenesis through downregulation of genes involved in the adipogenesis pathway and may be useful dietary supplement for the prevention of obesity. 


\section{P212 \\ Protective Effect of Lycopene on High Fat Diet-Induced Hepatic Steatosis and Involvement of MicroRNA-21 Mediated Repression of FABP7}

Jiyun Ahn, Hyunjung Lee, Chang Hwa Jung, Taeyoul Ha

Korea Food Research Institute

Non-alcoholic fatty liver disease (NAFLD) is a chronic disorder characterized by hepatic fat accumulation and abnormal lipid metabolism. Here, we investigated the protective effect of lycopene on high-fat diet-induced hepatic steatosis and fatty acid-induced intracellular lipid accumulation by miRNA regulation. C57BL/6J mice were fed high-fat diet with or without $0.05 \%$ lycopene for 8 weeks. Hepa 1-6 cells were treated with stearic acid (SA) after $24 \mathrm{~h}$ pretreatment with lycopene. Treatment of lycopene improved hepatic steatosis in high-fat diet fed mice and reduced intracellular lipid accumulation induced by SA in Hepa 1-6 cells. We demonstrated that miR-21 expression was decreased in livers from high-fat diet-fed mice and Hepa 1-6 cells treated with SA. Lycopene normalized the downregulation of miR-21, which led to the downregulation of fatty acid binding protein 7 (FABP7), a direct target of miR-21, at both the transcriptional and translational levels. This specific negative regulation of miR-21 was achieved by targeting the FABP7 3'UTR. Upregulation of miR-21 markedly blocked SA-induced intracellular lipid accumulation by blocking FABP7 expression. Moreover, silencing of FABP7 effectively reduced SA-evoked lipid accumulation in Hepa 1-6 cells. The results suggest that lycopene may be a useful functional compound for treating NAFLD by regulating hepatic lipid metabolism.

\section{P213}

\section{The Polymorphisms of the Vitamin D Receptor and Inflammatory Bowel Disease}

Tainá Nunes Rodrigues, Nathália Marques Barros, Márcia Soares Mota, Cyrla Zaltman

Universidade Federal do Estado Do Rio De Janeiro

The increased prevalence of inflammatory bowel disease (DII) associated with limitations in the knowledge of its etiology has brought attention to important modulator of the immune response associated with chronic inflammatory processes. One of the most important facts is that vitamin D can accumulate in the microenvironment of lymphoid organs, where it exerts specific autocrine and/or paracrine effects, and activated T cells can perform only the last vitamin D-activating reaction, hydrolyzing $25(\mathrm{OH}) \mathrm{D} 3$ to $1,25(\mathrm{OH}) 2 \mathrm{D} 3$, whereas macrophages and some dendritic cells (DCs) have both of the enzymes needed to convert vitamin $\mathrm{D}$ to $1,25(\mathrm{OH}) 2 \mathrm{D} 3$. Macrophages and activated DCs have the enzyme 1-alpha-hydroxylase, which promotes the local production of 1,25(OH)2D3, leading to paracrine effects. Vitamin D receptor (VDR) gene and vitamin D appear to be involved in increased susceptibility to autoimmune and inflammatory diseases, including the Crohn's Disease (CD) and
Ulcerative Colitis (UC). Furthermore, there is an increase of studies about de prevalence of several polymorphisms in the vitamin D receptor gene that my influence on the evoluation and risck of these diseases. The aim of this study was an update on the possible association of nutritional status of vitamin D, VDR gene polymorphisms and DII. We have done a review of the literature in the period from january 2000 to may 2012 includes articles that describe the action of vitamin D and VDR polymorphism in etiopathogenesis of DII. The results demonstrate that there is a required of vitamin D and the VDR for normal numbers of regulatory T-cells that have been shown to suppress experimental DII. There is also a possible association of genetic polymorphism of the type Apa I, Taq I and Bsm I in exon 8, and Fok I in exon 2. Prevalence of genotypic polymorphisms in the vitamin D receptor associated with IBD has yielded conflicting results, possibly due to ethnic differences in the populations studied. There is an increase in CD risk for Europeans carrying TaqI tt genotype and significant decrease in CD risk for all carriers of the ApaI "a" allele. The TaqI tt genotype is associated with susceptibility to both UC and CD in males. In conclusion, this study demonstrates that vitamin D exerts immunomodulating effects that may hold promise in many diseases, characterized by anti-Th1 and anti-Th17 effects, proTh2 effects, and induction of Tr1 cells. The development of IBD, as well as the role of impaired vitamin D.

\section{P214}

\section{Prolonged Exposure to Omega 3 Fatty Acids Improves Insulin Sensitivity and Modulates Genes Involved in Insulin Signaling Pathway in Mice}

Talita Romanatto, Bin Wang, Rui Curi, Jing X. Kang

University of São Paulo/ Harvard Medical School

Background and aims: A growing body of evidence suggests that the amount and type of fat included in the diet contribute to the development of insulin resistance and that the reduction of the tissue n-6/n-3 fatty acid ratio could effectively suppress the development of many chronic diseases, including inflammatory disorders, diabetes and neurodegenerative diseases. The fat- 1 transgenic mouse is capable of converting omega-6 (n-6) to omega-3 (n-3) fatty acids, leading to an increase in $n-3$ fatty acid content with a balanced n- $6 / n-3$ fatty acid ratio in all tissues, independent of diet. In the present study we investigate the effects of n-3 on insulin sensitivity as well as the expression of insulin pathway-related genes in aging mice.

Materials and Methods: The total number of C57BL/6 mice were divided in four study groups: wild-type at age 8 weeks $(n=4)$, fat- 1 at age 8 weeks $(n=4)$, wild-type at age 8 months $(n=4)$ and fat- 1 at age 8 months $(n=4)$. The tissue fatty acid composition was measured using gas chromatography (GC). After the treatment period, body mass, food intake and the plasma concentration of glucose and insulin were measured. Glucose tolerant testing (GTT), insulin tolerant testing (ITT) and heart gene expression by Real Time PCR were performed in all groups.

Results: Lipid analysis confirmed that $n-3$ tissue levels of fat- 1 mice are much higher than that of wild type mice. Fat- 1 mice at 8 months of age showed decreased body weight and food intake, 
decreased basal glucose and insulin levels and displayed an improvement in glucose tolerance and insulin sensitivity during GTT and ITT, respectively, compared to wild-type mice of the same age. Evidence for increased insulin related genes in the heart tissue of fat- 1 mice (age 8 months) was obtained by a 2-fold increase in mRNA levels for insulin receptor (IR) and protein kinase B (Akt) and 3-fold increase in mRNA levels for phosphatidylinositol 3-kinase (PI3K-p85) and nitric oxide synthase (e-NOS) compared with all other groups.

Conclusions: Aged fat-1 mice are more insulin sensitive than the other groups tested in this study, despite the age-related insulin resistance observed in the aged wild type mice. This suggests that a prolonged increase in n-3 fatty acid tissue content with a balanced of the n-6/n-3 fatty acid ratio may be an important approach for the prevention and treatment of insulin resistance and related chronic diseases.

Financial support: FAPESP (2009/50621-6).

P215

The Relationship Between FTO

Polymorphisms and Anthropometric and

Metabolic Profile in Individuals with MS Enrolled in a Nutritional Orientation Program

Tamiris Invencioni Moraes, Raquel Oliveira,

Rebecca Kristina Kiyokawa, Marcela Correia Sousa,

Alvaro Cerda, Mario Hiroyuki Hirata,

Cristina Moreno Fajardo, Egidio Lima Dorea,

Nagila R. Teixeira Damasceno, Monica Ines Elias Jorge,

Rosario D. Crespo Hirata

University of São Paulo

Background: Metabolic syndrome (MS) is a set of metabolic disorders characterized by central obesity, insulin resistance, dysglycemia, dyslipidemia, and hypertension, which are important risk factors for cardiovascular disease. Common polymorphisms in Fat Mass and Obesity Associated gene (FTO) have been found to be associated with obesity and MS, however there is limited information in our population.

Objectives: We have investigated the relationship between FTO polymorphisms and anthropometric and metabolic profile in individuals with MS enrolled in a nutritional orientation program (NOP) to reduce body weight.

Methods: Anthropometric, body fat composition and clinical data were obtained from 62 MS patients, before and after the NOP lasting nine weeks. Blood samples were also obtained to evaluate metabolic variables (lipid, glucose, leptin and other markers) and genomic DNA extraction. FTO polymorphisms rs1558902 T > A, rs17817449 T > G, rs9930506A > G, rs9939609T > A and rs8050136 $\mathrm{C}>\mathrm{A}$ were detected by Real Time PCR.

Results: Allele frequencies of FTO variants were similar to that found in European populations.

MS patients carrying FTO rs1558902 TT genotype had higher baseline body weight than the A allele carriers (TA + AA genotypes) $(\mathrm{p}=0,010)$. After NOP, carriers of FTO rs 1558902 A allele (TA + AA genotypes) had higher reduction of weight, body mass index (BMI) and abdominal circumference $(\mathrm{p}<0.05)$ than TT genotype carriers. In addition, MS patients carrying FTO rs9039609 TT genotype had greater reductions in body weight $(\mathrm{p}=0.010)$ than the allele A (AA + TA genotypes) carriers, after NOP. There was no relationship of SNPs with lipid profile, glucose, leptin and other markers.

Conclusions: These results are suggestive that the FTO 1558902 $\mathrm{T}>\mathrm{A}$ and rs9039609 $\mathrm{T}>$ A polymorphisms contribute with a reduction of body weight in response to a nutritional orientation program in MS patients.

Financial support: FAPESP.

\section{P216 \\ Methionine Imbalance in the Diet Affects the DNA Stability}

\author{
Tarsila D.U.H. Gomes, Alexandre Ferro Aissa, \\ Joana D. Arc C. Darin, Maria de Lourde Pires Bianchi, \\ Lusânia Maria Greggi Antunes \\ FCFRP/USP
}

Background: Environmental factors during intrauterine development, such as maternal diet, may alter the metabolism of the offspring. One of these nutrients is the amino acid methionine (Met) that is required for protein synthesis and for methyl donation to the DNA methylation process, especially in the early life development. It is metabolized by liver to homocysteine, a compound that might interfere with reactive oxygen species (ROS) levels and cause damages to the DNA. Therefore, it is important to evaluate how a Met imbalance may alter the DNA stability.

Objectives: This study aimed at evaluating the DNA damage induced by Met imbalance in mice, by the comet assay in hepatic and renal tissues.

Methods: Male mice offspring of dams, that previously fed different Met diets (control, $0.3 \%$ DL-Met, supplemented, $2.0 \%$ DL-Met), were treated with control, supplemented or deficient $(0 \%$ DL-Met) Met diets ad libitum for 18 weeks. Then, the animals were euthanized and the liver and kidney were collected to carry out the comet assay under alkaline conditions. The comet assay parameter used was the $\%$ tail DNA (CometAssay IV ${ }^{\mathrm{TM}}$ software) scored in 100 nucleoids per animal.

Results: The Met supplementation and deficiency in the offspring diet, regardless the maternal diet, increased $(p<0.05)$ the DNA instability in both tissues when compared to the offspring of control dams group treated subsequently with control diet.

Conclusions: The DNA damage induced by Met supplementation might be due to a ROS production increased in response to a homocysteine metabolism. Besides, it might be possible that occurred a saturation of the transulfuration pathway and the glutathione produced in this pathway did not neutralize all the ROS generated. The DNA instability caused by Met deficiency may be due to the uracil misincorporation in the genetic material when methyl group is depleted. Further studies related to genes expression may elucidate how Met imbalance can interfere in its metabolism cycle, and other biomarkers.

Financial support: CAPES/CNPQ (CNPQ 471078/2010-7). 


\section{P217}

\section{Characterization of the Fecal Levels of Bifidobacterium and Lactobacillus Species from Lean and Obese Adult Women}

\author{
Tatiana Fiche S. Teixeira, Lisiane Lopes Conceição, \\ Seppo Salminen, Kirsi Laitinen, Josefina Bressan, \\ Maria do Carmo Gouveia Peluzio
}

Universidade Federal de Viçosa

Gut microbiota can affect the gene expression of the host and may predispose to obesity. Characterization of gut microbiota from lean and obese individuals at species is scant, particularly in the case of Bifidobacterium and Lactobacillus. The identification of species that are naturally present in higher concentrations in lean individuals might direct the search for strains that can have higher potential to positively affect body weight or nutritional status management.

The aim of this study was to characterize specific Bifidobacterium and Lactobacillus species in feces from female adults.

This was a cross-sectional study in which seventeen lean (BMI $\left.19-24,99 \mathrm{~kg} / \mathrm{m}^{2}\right)$ and fifteen obese females $\left(\right.$ BMI $\left.>30 \mathrm{~kg} / \mathrm{m}^{2}\right)$ of similar age participated. Anthropometric and body composition measurements were assessed. Specific bacterial groups were analyzed in fecal samples by qPCR. Bifidobacterium genus, B. longum, Bifidobacterium catenulatum, Bifidobacterium bifidum, Bifidobacterium adolescentis, Bifidobacterium breve, Lactobacillus acidophilus, Lactobacillus paracasei, Lactobacillus plantarum, Lactobacillus rhmanosus and Lactobacillus casei species were characterized and compared regarding the prevalence and count number. Data are expressed as median (interquartile range). Anthropometric and body composition of lean and obese women were as follows: BMI 21.2 (20.6-21.9) vs 34.5 (32.8-36.7), waist (cm) 68 (66.5-72) vs 93.5 (89.5-97), body fat (\%) 22.7 (18-2.8) vs 37.5 (36.7-38.9) $(\mathrm{p}<0.05)$. Lactobacillus casei was the only bacterial specie not identified in the fecal samples from either group. The only specie that differed regarding the prevalence was Lactobacillus plantarum, whose presence was more common in the lean group (76\% vs $25 \%$, $p=0.005)$. A tendency for lower counts of $L$ plantarum in obese subjects was also observed $(p=0.06)$. B genus and $B$ longum counts were lower in obese subjects $(p<0.05)$. The other bacterial species analyzed did not differ between groups. B genus, B longum and $\mathrm{L}$ plantarum inversely correlated with weight, BMI and fat percentage $(\mathrm{p}<0.05)$. Specific species from the Bifidobacterium and Lactobacillus genus are naturally present in higher levels in lean women, and since increased numbers of $\mathrm{B}$ longum and $\mathrm{L}$ plantarum correlated with lower weight, BMI and fat percentage, these species might be explored in future human studies as potential probiotics for obesity management.

Financial support: FAPEMIG and academy of Finland (APQ0131310).

\section{P218 \\ Endotoxins and Chronic Diseases}

Tatiana Fiche S. Teixeira, Raquel Duarte M. Alves, Ana Paula Boroni, Viviane Silva Macedo, Maria do Carmo Gouveia Peluzio

Universidade Federal de Viçosa

The term endotoxin refers to any toxin associated with bacterial cells, more specifically to the lipopolisaccharide (LPS), a structural molecule of the outer part of the cell's wall of gram-negative bacteria. Gut microbiota is a huge reservoir of endotoxins and alteration of its composition has been associated with obesity and diabetes. LPS molecule comprises three domains: O-antigen, core $\mathrm{R}$ and lipid $\mathrm{A}$. The lipid A portion determines the biological activity of LPS. It has been verified that a chronic infusion of low levels of LPS in mice promoted a low grade inflammatory status, a key aspect of chronic diseases. Obese and diabetic patients show higher levels of circulating LPS. Specific receptors in the cell membrane of immune, epithelial and adipocytes cells known as toll-like receptors (TLR-4) interact with LPS and activate intracellular signaling cascades activating the nuclear factor kappa beta (NFkB). This transcriptional factor stimulates the expression of pro-inflammatory cytokines such as tumor necrosis factor alpha (TNF- $\alpha$ ) and interleukins (IL-1, IL-6 and IL-6), that maintains the low grade inflammation. Subclinical increment in the circulatory levels of endotoxins is associated with weight gain, insulin resistance, glucose intolerance and oxidative stress, similarly to the effects of a high fat diet (HF). This dietary pattern modifies the gut microbiota composition by increasing the population of gramnegative bacteria. It also leads to an increment in the intestinal permeability which might be a route to LPS to access the circulation. Besides, the HF also stimulates the formation of chylomicrons, which incorporates LPS from the intestinal lumen into its structure, transporting LPS through the lymphatic circulation. The type of dietary fat differently influences the endotoxin levels and consequently the gene expression of host cells. Different lipid sources (milk, palm, rapeseed or sunflower oil) were administrated to mice and it was shown that palm oil intake resulted in the most active transport of LPS toward tissues. Consequently, those mice had the highest inflammatory response and expression of the receptors TLR4 and CD4 in white adipose tissue. Thus, LPS seems to act synergistically with fatty acids on the secretion of proinflammatory cytokines, endotoxin transporters and inflammation. It is important to understand the link between the dietary fatty acids profile and endotoxemia in the chronic diseases development and management.

Financial support: FAPEMIG (CDS-APQ-01313-10). 
P219

\section{Nutrigenetics and Memory: the Interaction Between Zinc Intake and the Variability of the Gene SLC30A3 on Memory Scores in Aging}

\author{
Tatiane Jacobsen da Rocha, Pâmela Camini Constantin, \\ Cláudia Blehm, Camila Korb, Daiani Bamberg, \\ Andrea Guedes, Tainá Ludmila Ramos Fonseca, \\ Alcyr Oliveira Junior, Luciana Alves Tisser, \\ Fabiana Michelsen de Andrade, Marilu Fiegenbaum
}

Universidade Federal de Ciências da Saúde de Poa

Zinc is an essential micronutrient and plays roles in cell metabolism and gene expression, besides its important role in neurogenesis. The SLC30A3 gene encodes the zinc transporter protein 3 (ZnT3) and is expressed exclusively in brain regions like the hippocampus and neocortex, regions important for memory consolidation. The objective of this study is to evaluate the interaction between the variability in the gene SLC30A3 and zinc intake on the memory of volunteers over 50 years. For this cross-sectional study, 197 volunteers were selected from groups of elderly people in the region of the Vale dos Sinos (Rio Grande do Sul, Brazil) for which five types of memories were analyzed by tests of the WAIS-III and Weschesler Verbal Learning Test of Rey. These volunteers responded a food frequency recall, based on two periods of life: before age 40, and from that age until the present age. This food recall contained information on the frequency of consumption in 3 zinc-rich foods (beef cattle, chicken and fish). For statistical analysis food groups were grouped into two class of consumption alone (day vs twice a week or less). The determination of the genotypes of SLC30A3 gene polymorphisms (rs11126936 and rs73924411) was performed by allelic discrimination assays in real-time PCR. Nutrigenetic interactions were investigated using the factorial design, using the level of education (in years) and sex as co-adjustment factors for memory scores. All statistical analyzes were performed using SPSS version 19.0. It was possible to perceive an apparent interaction $(\mathrm{p}=0.050)$ between the past consumption of zinc and rs11126936 polymorphism of the SLC30A3 gene, we found that GG homozygotes who consumed foods rich in zinc daily, showed lower immediate verbal memory scores than $\mathrm{T}$ allele carriers $(-0.18 \pm 1.39$ vs. $+0.29 \pm 1.18)$ although this difference was not observed in individuals with less than the consumption of zinc daily. The power may interact with individual genetic profile and determine the susceptibility of an individual to develop greater degrees of memory deficit. However, to date, no study investigated the influence nutrigenetics consumption of zinc and SLC30A3 gene polymorphisms in memory deficits. The data presented in this study should be confirmed with increasing sample size, and indicate a possibility for further personalization of diet-related modulation of memory.

\section{P220 \\ Nutrigenetics Evaluation of the Interaction Between the Consumption of Tryptophan and Polymorphism Ins/Del in the SLC6A4 Gene and Its Influence on Memory Scores}

\author{
Tatiane Jacobsen da Rocha, Renata Biachi Marian, \\ Ana Paula Nazario, Camila Korb, Daiani Bamberg, \\ Pâmela Camini Constantin, Luciana Alves Tisser, \\ Alcyr Oliveira Junior, Fabiana Michelsen de Andrade, \\ Marilu Fiegenbaum \\ Universidade Federal de Ciências da Saude de Poa
}

The synthesis of brain serotonin may be modulated by offering dietetically macronutrients, among the main tryptophan. The serotonin transporter (5-HTT) is responsible for the reuptake of serotonin released into the synaptic cleft and is encoded by the gene SLC6A4. Some studies suggest the role of serotonin in modulating learning and memory processes, however, investigations on the interaction between the intake of foods rich in tryptophan and the gene SLC6A4 on memory scores missing in the literature are scarce. The objective of this study is to evaluate the interaction between the variability in the gene SLC6A4 and consumption of foods rich in tryptophan, on the memory of volunteers over 50 years. For this cross-sectional study, 197 volunteers were selected from groups of elderly people in the region of the Valley of the Bells, for which five types of memories were analyzed by tests of the WAIS-III and Weschesler Verbal Learning Test of Rey These volunteers responded a food frequency questionnaire, based on two periods of life: before age 40, and from that age until the present age. This food recall contained information on the frequency in the consumption of foods rich in tryptophan (egg, cassava, meat and cheese). The determination of genotypes of polymorphism ins/del (rs25531) of the SLC6A4 gene was performed by PCR assay - RFLP using the restriction enzyme. The influence of interactions on scores nutrigenéticas memory was tested using factorial designs, with level of education (in years) and sex as covariates. All statistical analyzes were performed using SPSS version 19.0. We detected a significant interaction $(p=0.012)$ between the genetic variant and the consumption of foods rich in tryptophan before age 40 scores on verbal learning ability Rey: individuals who reported infrequent consumption of tryptophan have these scores decreased only when homozygous for the long allele. Already in short allele carriers, the consumption of this nutrient did not affect the scores of learning ability. Traditionally nutrition provides general information about the nutritional needs of the population. However, from studies on nutrigenetics, the genetic variability of the individual or population subgroups may be used to promote adequate diet and optimal state of health. The data presented in this study should be confirmed with increasing sample size, and indicate a possibility for further personalization of diet-related modulation of memory. 


\section{P221}

\section{Nutrigenetics Interaction Between Selenium Intake and the Variability of the Gene 15 SEP on Memory Scores}

\author{
Tatiane Jacobsen da Rocha, Daiani Pires Bamberg, \\ Pâmela Camini Constantin, Camila Korb, Cláudia Blehm, \\ Paola Dornelles, Tainá Ludmila Ramos Fonseca, \\ Alcyr Oliveira Junior, Luciana Alves Tisser, \\ Fabiana Michelsen de Andrade, Marilu Fiegenbaum
}

Universidade Federal de Ciências da Saúde de Poa

Selenium is an important micronutrient and antioxidant, and studies show that selenium deficiency in the diet is associated with memory deficits in aging. However, nutrigenetic interaction on this characteristic has never been investigated. The gene of the selenoprotein $15 \mathrm{k}$ (SEP15) is responsible for the transport of about $50 \%$ of selenium found in plasma, and this concentration is influenced by diet. The objective of this study is to evaluate the interaction between the variability in gene SEP15 and consumption of selenium on the memory of volunteers over 50 years. For this cross-sectional investigation, 197 volunteers were selected from groups of elderly people in the region of the Vale do Sinos (Rio Grande do Sul, Brazil), for which five types of memories were analyzed by tests of the WAIS-III and Weschesler Verbal Learning Test of Rey. These volunteers responded a food frequency questionnaire, based on two periods of life: before age 40, and from that age until the present age. This food recall contained information on the frequency in the consumption of four foods rich in selenium (Brazilian nuts, cabbage, spinach and flaxseed). The determination of the genotypes of the SEP15 gene polymorphism (rs5859 rs5845 and rs561104) was performed by allelic discrimination assays in real-time PCR. The influence of nutrigenetic interactions on memory scores was tested using factorial designs, with level of education (in years) and sex as covariates. All statistical analyzes were performed using SPSS version 19.0. Of the five types of memory assessed only the immediate verbal memory was associated with genetic variability investigated (rs5845) and with selenium consumption. To this variant, it was seen that the selenium few frequent consumption was associated to decreased scores to this specific type of memory, but only in GG homozygotes, but this effect was not seen in allele A carriers $(p=0,035)$. Low selenium intake has been associated with various diseases, so understanding the interaction between the genetic profile of individual or population subgroups and selenium intake in disease susceptibility is essential. The data presented in this study should be confirmed with increasing sample size, and indicate a possibility for further personalization of diet-related modulation of memory.

\section{P222 \\ Yerba Maté (llex Paraguariensis) Aqueous Extract Modulates the Inflammatory Response in Wistar Rats Fed a High-Fat Diet}

\author{
Tatiane Mieko de Meneses Fujii, Monica Yamada, \\ Patrícia Silva Jacob, Maria Carolina Borges, \\ Lucas Carminati Pantaleão, Primavera Borelli, \\ Ricardo Ambrósio Fock, Julio Tirapegui, \\ Marcelo Macedo Rogero \\ University of Sao Paulo
}

Background: The consumption of high-fat diet (HFD), which is rich in saturated fatty acids, can increase body adiposity and contribute to the development of chronic diseases, such as obesity. Yerba maté (Ilex paraguariensis) has a high concentration of phenolic compounds, which can modulate the inflammatory response through inhibition of the nuclear factor $\kappa \mathrm{B}(\mathrm{NF}-\kappa \mathrm{B})$ signaling pathway.

Objectives: The purpose of this study was to investigate the effect of yerba mate aqueous extract (YMAE) on inflammatory response in rats fed a isocaloric HFD.

Methods: Male Wistar rats were fed a HFD ( $n=12 /$ group) or control $(\mathrm{CON}) \operatorname{diet}(\mathrm{n}=12 /$ group $)$ for 12 weeks. After this period, animals were randomly distributed in groups, which received water (CON1 and HFD1) or were treated with $1 \mathrm{~g} \cdot \mathrm{kg}-1$ of YMAE (CON2 and HFD2) for more 4 weeks. From blood, C reactive protein (CRP), lipid profile and free fatty acids levels were quantified. Carcass chemical composition was determined. Extraction of total proteins of soleus muscle was carried out and used for evaluating the expression and phosphorylation of c-Jun $\mathrm{N}$ terminal (JNK) and IkappaB kinase beta (IKK- $\beta$ ) and phosphorylation of NF- $\mathrm{KB}$ by the Western Blotting technique. Concerning the statistical analysis, comparisons between groups were initially performed by ANOVA. The alpha level was set for 0.05 . Significant differences were then compared using Tukey's test with a significance coefficient of 0.05 .

Results: The total energy intake did not differ between the groups. The treatment with YMAE reduced the percentage of weight gain, but adipose fat pads and plasma total fatty acid concentration did not differ between the groups. The CON2 group presented lower fat and higher lean mass percentage in comparison to the CON1 group. Plasma CRP, total cholesterol and triglycerides levels was lower in HFD2 group in comparison to the HFD1 group. There were no statistical differences between groups in relation to the expression and phosphorylation of JNK and IKK- $\beta$ and phosphorylation of $\mathrm{NF}-\kappa \mathrm{B}$.

Conclusions: YMAE promoted reduction in weight gain and in adiposity, which may be associated with reduction of inflammatory response. These results suggesting a potential benefit of yerba maté in reducing cardiometabolic risk.

Financial support: FAPESP (2009/54395-0) and CNPQ (470418/2009-5). 
P223

\section{Growth Hormone Secretagogue Receptor (GHSR) Gene Polymorphisms Association with Food Intake and Metabolic Features in Obese Children and Adolescents}

Thais Arthur, Eliana Frazzatto, Isabel Guazzelli, Simone Diniz, Sandra Villares

University of São Paulo Medical School, Brazil

Introduction: Ghrelin, a hormone-releasing peptide, enhances appetite and increases food intake in healthy men. The endogenous ligand ghrelin is the growth hormone secretagogue receptor (GHSR), and effects of ghrelin are mediated via the GHSR. Ghrelin and the GHSR are believed to have important roles in energy homeostasis and metabolic parameters. Common polymorphisms in GHSR have been associated with obesity. The GHSR gene 477G/A (rs572169) polymorphism is a good candidate for susceptibility to obesity, and subjects carrying the minor A allele had a higher dietary restraint and more disinhibition than subjects homozygous for the $\mathrm{G}$ allele.

Objective: Determine possible associations between the $477 \mathrm{G} / \mathrm{A}$ (rs572169) polymorphism of GHSR with energy intake, macronutrient intakes and metabolic features in obese children and adolescents.

Methods: A total of 347 obese children and adolescents $(37.2 \%$ boys, aged $10.6 \pm 1,4$ years, BMI $30.5 \pm 4.7 \mathrm{~kg} / \mathrm{m}^{2}$, ZBMI $2.3 \pm 0.3$, $46.3 \%$ pubertal) were genotyped for GHSR by PCR. Metabolic measures were obtained and. energy and macronutrient intakes were assessed by using 3-day food records. The statistical analysis was performed with Independent-samples T test by SPSS software.

Results: The population was in Hardy-Weinberg equilibrium (GG: $67.4 \%$, GA:29.7\%, AA:2.9\%, p = 0.74). GG children had higher energy intake that children carrying the A allele (GA + AA) (2127.7 \pm $641.2 \mathrm{Kcal}$ vs. $1815.3 \pm 562.0 \mathrm{Kcal}, \mathrm{p}=0.006)$ and carbohydrate intake $(261.0 \pm 86.9 \mathrm{~g}$ vs. $218.0 \pm 71.5 \mathrm{~g}, \mathrm{p}=0.005)$. GG carrier had also a higher total cholesterol $(160.7 \pm 31.2 \mathrm{mg} / \mathrm{dL}$ vs. $152.5 \pm 27.4$ $\mathrm{mg} / \mathrm{dL}, \mathrm{p}=0.02)$, higher LDLc $(98.8 \pm 27.1 \mathrm{mg} / \mathrm{dL}$ vs. $89.7 \pm 25.7$ $\mathrm{mg} / \mathrm{dL}, \mathrm{p}=0.003)$ and lower HDLc $(41.1 \pm 8.8 \mathrm{mg} / \mathrm{dL}$ vs. $43.5 \pm$ $10.1, p=0.03$ ) when compared with children carrying the A allele.

Conclusions: The results support the notion that the studied 477 G/A (rs572169) polymorphism of GHSR may be involved in obesity, food intake and with a more severe metabolic profile in obese children and adolescents. Our findings also suggest a complex genotype - environmental interactions on obesity risk.

\section{P224}

\section{Growth Hormone Secretagogue Receptor (GHSR) Gene Polymorphisms Association with Metabolic Features in Obese Children and Adolescents}

\author{
Sophie Deram, Eliana Frazzatto, Isabel Guazzelli, \\ Sandra Villares, Thais Arthur \\ University of São Paulo Medical School, Brazil
}

Introduction: Ghrelin, hormone-releasing peptide, enhances appetite and increases food intake in healthy men. The endogenous ligand ghrelin is the growth hormone secretagogue receptor (GHSR), and effects of ghrelin are mediated via GHSR. The GHSR system plays significant role in regulating the process of obesity and type 2 diabetes that are important components of metabolic syndrome (MS). Therefore, the GHSR gene 171C/T (rs495225) polymorphism is a good candidate for susceptibility to obesity and excellent for studying MS.

Objectives: To assess the frequencies of 171C/T (rs495225) polymorphism of GHSR in obese children and adolescents (OCA) as compared to normal weight healthy controls and to investigate that polymorphism are associated with MS and features metabolic in OCA.

Methods: A total of 428 Brazilian children, 91 normal weight healthy (43.9\% boys, aged $10.6 \pm 1.5$ years, BMI $17.0 \pm 2.4 \mathrm{~kg} / \mathrm{m}^{2}$, ZBMI $-0.3 \pm 0.9,40.6 \%$ pubertal) and 337 obese $(35.5 \%$ boys, aged $10.7 \pm 1.4$ years, BMI $30.6 \pm 4.7 \mathrm{~kg} / \mathrm{m}^{2}$, ZBMI $2.3 \pm 0.3,46.3 \%$ pubertal, $43.0 \%$ with MS) participated into the study. The polymorphic region of GHSR gene was amplified by polymerase chain reaction (PCR) using primers. MS was diagnosed by Adult Treatment Panel III (ATP III) adapted for children. The statistical analysis was performed with ANOVA by SPSS software.

Results: Genotype frequencies did not significantly differ between normal weight controls and obese children $(\mathrm{X} 2=0.66$, d.f. $=$ $2, \mathrm{p}=0.7)$. However, obese patients carrying the TT genotype in $171 \mathrm{C} / \mathrm{T}$ polymorphism had significantly higher values of HOMA $(4.21 \pm 0.19$ vs $3.66 \pm 0.26, p=0.02)$, higher frequency of MS $(44.1 \%$ vs $15.2 \%, \mathrm{p}=0.04)$, hypertriglyceridemia $(47.7 \%$ vs $14.1 \%, \mathrm{p}<$ $0.001)$ and a tendency for hyperglycemia $(56.5 \%$ vs $17.4 \%, \mathrm{p}=0.08)$ as compared to this carrying the $\mathrm{CC}$ genotype.

Conclusions: The results support the notion that the studied 171C/T (rs495225) polymorphism of GHSR might be a genetic risk factor for MS in OCA. Our findings also suggest a complex genotype- environmental interactions on obesity risk. 
P225

\section{Acute Physical Exercise Reduces Food Intake and Activates Tub, Insulin and Leptin Signaling Probably Via Hypothalamic IL-6 Action in Mice}

Thayana Oliveira Micheletti, Paula Gabriele Fernandes Qua resma, Lais Weissmann, Gustavo Duarte Pimentel,

Tanyara Silva Baliane, Manoela Ramalho,

Nayara Reencober, Andressa de Cassia dos Santos, Mario José Abdalla Saad, Patricia Oliveira Prada

Universidade Estadual de Campinas

Background: It is known that the regulation of food intake is mediated by the central nervous system (CNS) and insulin and leptin have a major role in this process. Studies showed that the protein Tub is express in hypothalamic areas which regulate food intake and energy expenditure. Deletion or mutation of Tub induces obesity and insulin resistance. Furthermore, studies from our group demonstrated that Tub is a substrate of insulin and leptin signaling in mice hypothalamus. Currently, it is known that physical exercise increases interleukin 6 (IL-6) levels and IL-6 may regulate energy homeostasis by acting in the hypothalamus.

Aim: To investigate the effect of acute physical exercise and hypothalamic IL-6 injections in the regulation of food intake, and insulin or leptin induced Tub, IR and Obr phosphorylation in the hypothalamus and in the adipose tissue of control mice.

Methods: Adults C57BL6/J mice fed with chow were cannulated in order to receive intracerebroventricular (ICV) injections of insulin, leptin or saline. A group of mice was submitted to 2 hours of exercise (swimming) and another group was not exposed to exercise (sedentary). Sedentary mice received saline or IL-6 peptide and exercised mice received saline or IL- 6 antibody. One hour after treatment mice received insulin, leptin or saline ICV and the food intake, tub phosphorylation in hypothalamus and adiposity were measured.

Results: We observed a decrease in food intake in the exercised group treated with insulin and leptin. However, IL-6 antibody blocked this effect. Decreased food intake was accompanied with an increase in tub, IR and Obr phosphorylation in the hypothalamus. Similarly to hypothalamus, there was an increase in tub, IR and Obr phosphorylation after insulin and leptin ICV injections in the epididimal adipose tissue of exercised and IL-6 peptide treated mice. In addition, epididimal adipose mass was decreased and hormone-sensitive lipase (HSL) protein levels was increased in this tissue, suggesting a possible increase in lipolysis. No differences were found in adipose tissue mass and HSL protein levels in exercised and IL-6 antibody treated mice.

Conclusions: Our results suggest that IL- 6 and physical exercise are able to induce reduction intake food, in addition to modulate Tub, IR and Obr phosphorylation in hypothalamus and in adipose tissue and may play a role in lipolysis.

Financial support: FAPESP (2010/18918-6).

\section{P226 \\ Anticarcinogenic Properties of Zinc and Selenium}

Thiago Leal B. Hipólito, Laís Lima Castro, Kyria Jayanne C. Cruz, Sueli Maria T. Lima, Maria do Carm o Carvalho Martins, Betânia Jesus S.A. Freitas

Federal University of Piauí

The completion of the human genome project has contributed to a major breakthrough in research aimed at finding answers about the relationship between the pathogenesis of chronic diseases and nutrition, more specifically on the possible interactions between genes and nutrients. Cancer is a disease in which cells with genetic alterations grow abnormally, invading other tissues and losing its original function. Evidence has shown that metabolic disorders are common in cancer cells, leading to increased oxidative stress. The increasing in production of reactive oxygen species associated with low antioxidant activity has been linked to several cancers. In recent decades, studies have been conducted with a view to clarify the involvement of micronutrient antioxidants and anticarcinogenic mechanisms in cancer development. Zinc in particular has been the element of greatest interest to most researchers. This mineral is involved in biochemical processes in antioxidant defense by being structural component of the superoxide dismutase and metallothionein, it also acts as a transcription factor and in activities of enzymes involved in DNA and RNA synthesis such as RNA and DNA polymerase, and thus seems to have effect inhibitory on the growth of neoplastic cells. Selenium, micronutrient antioxidant, can function as an antimutagenic agent, preventing malignant transformation of normal cells. This protective effect of selenium was first associated with its presence in glutathione peroxidase and thioredoxin reductase, enzymes that are known to protect DNA and other cellular components from oxidative damage. Some mechanisms have been suggested to explain the anticarcinogenic effect of selenium: the action of selenoenzymes in reducing DNA damage, oxidative stress reduction, inflammation reduction, detoxification, improved immune response, increased tumor suppressor protein $\mathrm{p} 53$, inactivation of protein kinase $\mathrm{C}$, changes in DNA methylation, blocking the cell cycle, inducing apoptosis of cancer cells and inhibition of angiogenesis. Despite evidence from epidemiological and laboratory anticarcinogenic properties these minerals, further studies should be conducted in order to better clarify the mechanisms of action of these nutrients in the genesis and prevention of cancer. 
P227

\section{Dietary Compounds with Anticarcinogenic Properties}

Thiago Leal B. Hipólito, Laís Lima Castro,

Kyria Jayanne C. Cruz, Sueli Maria T. Lima, Maria do Carm

o Carvalho Martins, Betânia Jesus S.A. Freitas

Federal University of Piauí

Several epidemiologic and experimental investigations have shown that bioactive compounds in food products (CBAs) may decrease the risk of many kinds of cancers. The CBAs present in food may act in different ways, both as regard to physiological targets as their mechanisms of action. The resveratrol found in peanuts, blueberries, grapes and plant species have antioxidant, anti-inflammatory, anticancer and cardioprotective. The mechanisms that explain these properties relate to the power to modulate gene expression by inhibiting the activation of Nuclear Factor Kappa B (NF-kB), the Activator Protein 1 (AP-1), of Inducible Nitric Oxide Synthase (iNOS) of Jun$\mathrm{N}$-terminal Kinase (JNK) and 5-lipoxygenase (5-LOX), promoting improvement of inflammatory processes and oxidative stress and suppressing the progression of tumors. The limonoids present in citrus fruits (citron, orange and lemon) whose main mechanism of action is the induction of glutathione-S-transferase (GST), an enzyme that promotes the conjugation of carcinogens that have suffered previous bioactivation by phase I enzymes in the metabolism of xenobiotics within the cytochrome $\mathrm{P} 450$, and as a result the decrease in toxicity of mutagenic substances. Quercetin (apple and citrus), the tyrosol (olive oil) and lycopene (tomatoes, watermelon and guava) inhibit in vitro gene the expression of COX-2 and iNOS, and, by decreasing the translocation of the transcription factor NF- $\kappa B$ from the cytoplasm to the nucleus, thereby reducing the inflammatory response. Minerals such as manganese, magnesium, zinc, copper and molybdenum may protect DNA, as well as present immunomodulatory functions, but little is known about specific antineoplastic properties. Calcium plays an important role antineoplastic. In the digestive tract, this mineral inhibits proliferation and increased differentiation of intestinal cells, inhibits activation of the enzyme ornitinadescarboxilase and promotes the activation of apoptosis of tumor cells through both the activation of caspase enzymes, which foster nuclear disintegration and organelles, as the activation of transglutaminases. From these literature data, it becomes possible to identify the importance of nutrigenomics as a tool to evaluate interactions between nutrients and bioactive food compounds in modulating gene and, especially, in contributing to the reduction of cancer.

\section{P228 \\ Hypolactasia in Adult: Genetic Test as a Comfortable Diagnostic Method}

Thiago Leal B. Hipólito, Artemízia Francisca Sousa, Julianne Viana F. Portela, Laís Lima Castro,

Livio Rodrigues Leal, Claricy Barbosa Leal, Livia Patrícia Ro

drigues Batista, Kyria Jayanne C. Cruz, Sueli Maria T. Lima

Federal University of Piauí

Hypolactasia is defined by the decline in enzymatic activity of the lactase, which affects a major part of the population after weaning as a irreversible and genetically programmed process, causing primary lactose malabsorption. In consequence, bacteria in the colon metabolize non digested lactose within the intestine lumen producing hydrogen, carbon dioxide and methane, leading to abdominal bloating and cramps, flatulence and diarrhea. Advances made on researches have shown that this disturb is associated with a polymorphism on the LCT-13910C > T nucleotide, the DNA region responsible for lactase persistence or nonpersistence. The diagnosis of lactose intolerance has been difficult to establish because of unsatisfactory diagnostic methods, including the hydrogen breath test, considered gold-standard. The validation of the LCT-13910C $>$ T polymorphism prompted the use of genetic test as screening test to diagnose hypolactasia. Its mechanism is based on measuring the lactase activity with a Quick Lactase Test kit with a sensitivity and specificity of $100 \%$ and $86 \%$ respectively, when compared to the hydrogen breath test. It also offers other advantages, as not requiring a pre-exam diet, lactose tests or fasting and being cheaper and more comfortable for the patient. Thus, facing these facts on literature review, genetic test can be used as a new available tool to diagnose hypolactasia among a population of patients suspected of lactose malabsorption.

\section{P229}

\section{Sphingosine 1-Phosphate Receptor 1/STAT3 Signaling Is Crucial for Regulation of Food Intake in Lean and Obese Rodents}

\author{
Vagner Rodrigues Silva, Érico Moreto Lins, \\ Luciene Lenhare, Thayana Oliveira Micheletti, \\ Guilherme Z. Rocha, Carlos K. Katashima, \\ Gustavo Duarte Pimentel, Patrícia Oliveira Prada, \\ Joseane Morari, Dennys Esper Cintra, José Rodrigo Pauli, \\ Lício Augusto Velloso, Mário José Saad, \\ José Barreto Carvalheira, Eduardo Rochette Ropelle \\ Department Of Internal Medicine, UNICAMP-Brazil
}

Rationale: Food intake and energy expenditure are regulated by neurons expressed in the hypothalamus. The leptin signaling (Jak2/ STAT3) is crucial for appetite regulation and energy expenditure. Recently, the sphingolipid S1P (sphingosine-1-phosphate) through of receptor S1PR1 (sphingosine-1-phosphate receptor-1) was described to act as a potent activator of leptin pathway in tumor cells. Therefore, we sought to investigate the effects of receptor S1PR1 on the activa- 
tion of Jak/STAT signaling in hypothalamus of lean and obese rodents.

Methods: Western blot, immunohistochemistry and the dissection of the hypothalamic regions techniques were combined to characterize the expression of S1PR1 in hypothalamus of lean and obese (diet-induced obesity) male Wistar rats and C57BL6/J and ob/ob mice. The intracerebroventricular (ICV) injection of S1P was performed to measure the food intake and to evaluate the leptin signaling in hypothalamus of both lean and obese rats. For statistical analysis were used the test $t$ Student and ANOVA one-way.

Results: Initially, we observed higher expression of S1PR1 in hypothalamus of rats when compared with other tissues, such as muscle, liver, heart and adipose tissue. The dissection of several hypothalamic nuclei showed an increased expression of S1PR1 in arcuate and ventromedial nuclei when compared to others hypothalamic area. Using the double staining in the immunohistochemistry approach, we observed that most neurons expressing S1PR1 in arcuate and ventromedial nuclei were shown to possess pSTAT3. Interestingly, the ICV infusion of S1P reduced the food intake dose-dependent manner in lean rats. The immunoprecipitation and Western blotting analysis showed a strong association of S1PR1/Jak2 and persistent phosphorylation of STAT3 in hypothalamus. In the other hand, the ICV infusion of curcubitacin, a pharmacological inhibitor of STAT3, abolished the effects of S1P on food intake. Furthermore, in obese rats and ob/ ob mice was found lower S1PR1 expression and leptin resistance in hypothalamus. Besides, the S1P ICV injection was able to activate Jak2/STAT3 pathway, to reduce the food intake and potentiate the leptin effects in obese rats.

Conclusions: The present study demonstrated that S1PR1 is able to control food intake through the interaction with the Jak2/ STAT3 pathway in the hypothalamus. In addition, S1P administration was sufficient to potentiate the anorexigenic effect of leptin in obese rodents.

Financial support: FAPESP (11/13828-1).

\section{P230}

\section{Nutritional Recovery Promotes Hypothalamic Inflammation in Adult Life}

\author{
Hellen Barbosa Farias Silva, Ana Paula Carli, \\ Katarine Barbosa Cardoso, Letícia Martins Ignacio-Souza, \\ Silvia Regina Lima Reis, Andressa Coope, \\ Marise Auxillia Barros Reis, Márcia Queiroz Latorraca, \\ Marciane Milanski, Vanessa Cristina Arantes
}

Universidade Federal de Mato Grosso

Background: Epidemiological studies have demonstrated a relationship between low birth weight and obesity in adult life. Low protein diet during important phases of life promotes hyperphagia, and the hypothalamus is an important regulator of food intake.

Objectives: We aimed to assess whether protein restriction in fetal life and after nutritional recovery during lactation alters the expression of hypothalamic cytokines (IL1- $\beta$ and TNF- $\alpha$ ), neuropeptides (POMC and NPY) and the AKT signaling.

Methods: Rats were divided into three groups: a control group (C) consisting of rats born to and suckled by dams fed a control diet during pregnancy, lactation and after weaning, a recovered group (R) consisting of the offspring of dams fed the LP diet during pregnancy but fed the control diet during lactation and after weaning, and an LP group consisting of the offspring of dams fed a LP diet during pregnancy, lactation and after weaning. After $120 \mathrm{~d}$ old were evaluated nutritional and biochemical parameters, gene expression involved in hypothalamic inflammatory pathway, as well as, AKT signaling in different tissues.

Results: The relative food intake was greater in LP and $\mathrm{R}$ rats than $\mathrm{C}$ rats. The liver glycogen content was similar in $\mathrm{R}$ and $\mathrm{LP}$ rats and both showed greater liver glycogen concentration than the $\mathrm{C}$ group. Serum albumin was diminished in LP. Both R and LP rats, showed diminished serum leptin concentrations than $\mathrm{C}$ rats. Mean total areas under the $\Delta \mathrm{G}$ were similar in $\mathrm{R}$ and $\mathrm{C}$ rats and higher than $\mathrm{LP}$ rats. The HOMA-IR index was decreased in $\mathrm{R}$ and $\mathrm{C}$ compared to the LP group. In contrast, the Kitt in $\mathrm{R}$ was similar from that in $\mathrm{C}$, and both lower than in LP rats. AKT phosphorylation in the hypothalamus and liver were similar in $\mathrm{R}$ and $\mathrm{C}$ rats and higher than LP. In muscle, AKT phosphorylation was higher in R and LP than the C rats. PCR array in hypothalamus showed that, 52 inflammatory genes were upregulated in $\mathrm{R}$ and 59 down-regulated in LP group compared to $\mathrm{C}$ group. TNF- $\alpha$ expression was higher in R group, whereas IL- $\beta$ did not differ among three groups. Hypothalamus of LP showed greater NPY mRNA expression than of $\mathrm{C}$ rats. The $\mathrm{R}$ group showed higher POMC mRNA expression in relation to both LP and $\mathrm{C}$ groups.

Conclusions: Our results suggest the nutritional recovery contributed to hypothalamic inflammation and the protein restriction during all life promotes hyperphagia, but not hypothalamic inflammation.

Financial support: FAPEMAT (Processo No. 282448/2010).

\section{P231 \\ The Cytoprotective Role of Short Chain Fatty Acids in Acute Kidney Injury Model Involves Modulation of Inflammatory Process and Epigenetic Modifications}

Vinicius Andrade-Oliveira, Mariane T. Amano, Matheus Correa-Costa, Angela Castoldi, Pedro M. MoraesVieira, Jean S. Peron, Énio J. Bassi, Danilo C. Almeida, Clarice Origassa, Meire I. Hiyane, Caroline M. Ferreira, Marco A. Vinolo, Rui Curi, Niels Olsen Camara

University of Sao Paulo

Background: Short chain fatty acids (SCFA) such as acetate, propionate and butyrate, are the end-products released from the fermentation of complex carbohydrates by anaerobic bacteria of the intestinal tract, for which have been ascribed anti-inflammatory roles.

Objectives: to evaluate the role of SCFA in model of acute kidney injury whereas inflammation plays a major role.

Methods: Mice were submitted to model of AKI injury (ischemia-reperfusion injury (IRI). SCFA were given individually (200 $\mathrm{mg} / \mathrm{kg}$ ) or in pool (200 mg/kg of each), $0.5 \mathrm{~h}$ before ischemia and at the time of reperfusion. After $24 \mathrm{~h}$, samples were collected for analyses. A p value was considered significant when $\leq 0.05$.

Results: SCFA induced better renal function, when administered isolated or in pool when compared to untreated group, $(n=5$ for all 
groups and $\mathrm{p}<0.0001$ ), being acetate associated with the best protection. This improvement was due to lower protein levels of inflammatory molecules as IL1- $\beta$, IL-6 and MCP-1. A reduction in the influx of macrophages, activated dendritic cells and neutrophils was also seen in SCFA-treated animals. Interestingly, an amelioration of the systemic inflammatory response was also achieved, better seen by the reduced serum levels of MCP-1, IL1A and RANTES. All together, these data was corroborated by a decreased activation of NF- $\kappa$ B pathway. The level of apoptosis also reduced in kidney of animals treated with SCFA. In vitro, it was observed that these SCFA modulate of the activation and the generation of bone marrow derived dendritic cells. LPS-pulsed dendritic cells treated with SCFA also induced less lymphocyte $(\mathrm{CD} 4+$ and $\mathrm{CD} 8+)$ proliferation in allogeneic context and SCFA treatment inhibited lymphocyte proliferation under specific or polyclonal stimuli. In a kidney epithelial cell line, the SCFA treatment reduces NFK-b activation in inflammatory conditions. We observed that treatment with acetate modulated the expression of genes related to chromatin modification (epigenetic regulation) in the kidneys of animals submitted to IRI. Finally, the treatment with acetate-producing bacteria ameliorates kidney function after IRI injury.

Conclusions: The SCFA is a promising tool in the protection of the AKI.

Support: FAPESP (process number: 2011/01016-2 and 12/02270-2), CNPq (LIA, CNPq/Inserm), Complex Fluids INCT, and Cepid-Fapesp.

P232

Muscle Gene Expression in Response to Sepsis and Supplementation with Free L-Glutamine Plus L-Alanine or L-Alanyl-LGlutamine

Vinicius Fernandes Cruzat, Lucas Carminatti Pantaleão, Pau lo Ivo Homem Bittencourt Jún, Julio Orlando Tirapegui

University of São Paulo

Background: Sepsis is the leading cause of death in intensive care units (ICUs) in the world and in Brazil. The availability of glutamine is reduced in sepsis, possibly altering the gene expression response of many tissues, especially skeletal muscles, which seems to correlate with increased morbidity and mortality. Oral or enteral supplementations with L-glutamine dipeptides, such as L-alanyl-Lglutamine (DIP) or solutions containing L-glutamine plus L-alanine, both in the free form (ALA + GLN) can represent an efficient way to provide glutamine for the body in some catabolic situations.

Objectives: We investigated the effects of ALA + GLN and DIP supplementation on skeletal muscle gene expression in a model of sepsis.

Methods: C57B6 mice were subjected to endotoxemia (LPS, Escherichia Coli, $\left.5 \mathrm{mg} \cdot \mathrm{kg}^{-1}\right)$ and supplemented orally with L-glutamine $\left(1 \mathrm{~g} . \mathrm{kg}^{-1}\right)$ plus L-alanine $\left(0.61 \mathrm{~g} \cdot \mathrm{kg}^{-1}\right)$ both in the free form (GLN + ALA-LPS) or DIP (1.49 g. $\left.\mathrm{kg}^{-1}\right)$ (DIP-LPS). Plasma and skeletal muscle concentrations of glutamine were evaluated by ELISA kit, and the expression rate of 41 genes in gastrocnemius muscle was analyzed by Real Time-PCR Array.
Results: LPS group exhibited lower concentration of glutamine in plasma and gastrocnemius muscle $(\mathrm{p}<0.05)$, when compared to control and supplemented groups. We detected a differential skeletal muscle gene expression profile in LPS group with up or down-regulation of $68.3 \%$ genes when compared to control. Among these genes, the expression of $31.7 \%$ was at least partially recovered in GLN + ALA-LPS and DIP-LPS groups. MRNA of genes related to inflammation (E.g: NFkB, Myd88, IRAK) and that codify for chaperone proteins (E.g: HSF1 and HSPs) were more expressed in LPS group than in controls, GLN + ALA-LPS and DIP-LPS groups $(\mathrm{p}<0.05)$. Significantly less mRNAs of genes related to protein synthesis (E.g: Eif4e, mTOR) and more mRNAs of genes related to protein breakdown (E.g: Foxo3, Trim63, Casp 1 and 3) were observed in LPS group, compared to controls $(\mathrm{p}<0.05)$. However, genes related to protein synthesis and degradation did not differ in LPS group compared to GLN + ALA-LPS and DIP-LPS groups.

Conclusions: Our results indicate that L-glutamine plus L-alanine in the free form or L-alanyl-L-glutamine dipeptide represent an efficient way to provide glutamine for the body, modifying muscle gene expression profile related to inflammatory response, which provide additional protection during endotoxemia.

Financial support: FAPESP and CNPQ (09/52853-1).

\section{P233 \\ Involvement of the Complexes 1 and 2 of MTOR in the Changes in Gene Expression Induced by LPS in Macrophages (Peritoneal and Raw 264.7) and 3T3I1 Adipocytes}

\author{
Vivian Almeida Paschoal, Thiago Belchior Oliveira, \\ Juliana Magdalon, Rui Curi, William Tadeu Festuccia \\ ICB - USP
}

Introduction: Previous studies found an increased activity of the mTOR complex 1 (mTORC1) in some cell types after treatment with lipopolysaccharide (LPS) and cytokines such as TNF-alpha and IL1beta. In this study we evaluated the contribution of mTOR complexes 1 and 2 to the changes in gene expression induced by LPS treatment of peritoneal and RAW 264.7 macrophages and 3T3L1 differentiated adipocytes.

Methods: C57BL6 peritoneal mouse macrophages and RAW 264.7 cells treated with LPS $(5 \mathrm{mg} / \mathrm{mL})$ in association or not with rapamycin (mTORC1 $100 \mathrm{nM}$ inhibitor) and torin (250 nM inhibitor mTORC1 and 2) for 4 hours. 3T3L1 cells treated with LPS (100 ng/ $\mathrm{ml})$ in association or not with rapamycin $(100 \mathrm{nM})$ or Torin $(250 \mathrm{nM})$ for $24 \mathrm{~h}$ were assessed for mRNA levels from several cytokines by QPCR.

Results: LPS treatment significantly increased peritoneal macrophages mRNA levels of COX2, IL1 $\beta, \mathrm{TNF} \alpha$ and MCP1 and reduced those of lipin. Among these genes, the upregulation in MCP-1 and TNF $\alpha$ mRNA levels induced by LPS was partially (MCP-1) or completely $(\mathrm{TNF} \alpha)$ attenuated by $\mathrm{mTORC} 1$ inhibition with rapamycin, while LPS-induced IL1 $\beta$ upregulation was impaired by inhibition of mTORC1 and 2 by torin. Similarly, treatment with either rapamycin or torin blocked the reduction of lipin levels induced by LPS. In RAW 264.7 cells, LPS significantly increased mRNA levels of COX2, 
IP10, IL1 $\beta$, TNF $\alpha$ and MCP1. In contrast to the peritoneal macrophages, however, treatment with torin attenuated the increase in IL-1 $\beta$ induced by LPS alone. In 3T3L1 adipocytes, LPS significantly increased mRNA levels of COX2, MCP1, PAI1. In contrast to macrophages, however, LPS induced COX2 upregulation was completely attenuated by Torin and rapamycin. Torin, also attenuated LPS induced upregulation of PAI1 and resistin mRNA levels.

Conclusions: Our data suggest that complexes 1 and 2 of mTOR seem to actively participate in the modulation of gene expression associated with treatment with LPS in macrophages and 3T3L1 cells. RAW 264.7 cells respond differently compared to peritoneal macrophages when treated with rapamycin and torin.

Financial support: FAPESP.

\section{P234}

\section{N-3 Fatty Acids Protects from the Glucose Intolerance Associated with High Fat Feeding by Activating PPAR?}

\author{
Thiago Belchior Oliveira, Juliana Magdalon, \\ Vivian Almeida Paschoal, Talita Farias, André Marette, \\ Rui Curi, William Tadeu Festuccia \\ ICB - USP
}

Introduction: Supplementation with n-3 fatty acids was previously demonstrated to have beneficial actions against the development of chronic metabolic diseases such as diabetes and obesity. In this sense, genetically modified mice (FAT-1) with an increased endogenous production of n-3 from n- 6 fatty acids due the transgenesis of a fatty acid desaturase were protected from the development of diet-induced obesity. Here we tested the hypothesis that n-3 fatty acids protect from the development of diet-induced obesity and insulin resistance by activating the nuclear receptor peroxisome proliferator activated receptor $\gamma(\operatorname{PPAR} \gamma)$.

Methods: Wild type and FAT1 C57BL/6 mice fed with high-fat diet supplemented or not with a PPAR $\gamma$ antagonist GW9662 $(0.1 \%$ $\mathrm{w} / \mathrm{w})$ for 8 weeks were evaluated for body weight gain, adiposity, glucose tolerance (GTT) and adipose tissue glucose metabolism.

Results: FAT-1 mice were protected from obesity and glucose intolerance (GTT) associated with high fat feeding. Pharmacological PPAR $\gamma$ inhibition, however, impaired glucose tolerance of FAT-1 mice to levels close to high fat fed wild type mice. Surprisingly, this impairment of glucose tolerance induced by PPAR $\gamma$ inhibition in FAT-1 mice was not associated with changes in adiposity or glucose incorporation into triacylglycerol and its components: fatty acids and glycerol.

Conclusions: Our data suggest that the beneficial effects of n-3 fatty acids against the development of the glucose intolerance associated with high fat feeding are, at least in part, associated with an activation of PPAR $\gamma$ by these fatty acids.

Financial support: FAPESP.

\section{P235}

\section{Association of Vitamin D Receptor Gene Polymorphisms with Blood Pressure}

Vivian Cristina Garcia, Natielen Jacques Schuch, Sandra R.G. Ferreira, Lígia Araújo Martini

University of Sao Paulo

Findings suggest that vitamin D receptor (VDR) gene polymorphisms are associated with higher susceptibility to several diseases, including hypertension. To evaluate the potential association of presence of polymorphisms in VDR gene with high blood pressure in adults. In a cross-sectional study, 242 individuals (62\% women) were evaluated. Anthropometric measurements, blood pressure and a fasting blood sample were obtained. Serum concentration of $25(\mathrm{OH}) \mathrm{D} 3$ was measured by HPLC and iPTH by electrochemiluminescence. BP was obtained by mean of two measures. Vitamin D insufficiency was defined by $25(\mathrm{OH}) \mathrm{D} 3 \leq 75 \mathrm{nmol} /$. The VDR rs1544410 (BsmI) and rs2228570 (FokI) polymorphisms were performed by allele specific PCR. Individuals with chronic kidney disease were excluded. HardyWeinberg equilibrium test was performed. Statistical analysis was performed by SPSS (v.17.0), significance level was $p<0.05$. Mean age 51(14) years, BMI 29(6) $\mathrm{kg} / \mathrm{m}^{2}$, waist circumference $98(14) \mathrm{cm}$ and iPTH 40(18)pg/mL. Mean blood pressure was 130/80(18/11) $\mathrm{mmHg}$. Mean vitamin D was 58(17)nmol/L. Vitamin D insufficiency was present in $82 \%$ of the sample and $54 \%$ of individuals had blood pressure above of 140 and/or $90 \mathrm{mmHg}$. Genotype variation of BsmI and FokI was, respectively: BB, 14\%, Bb, 41\%, bb, $45 \%$ and FF, $9 \%$, Ff, 53\%, ff, 38\%. No differences were observed between BsmI and FokI polymorphism and BMI, waist circumference, vitamin D, PTH and blood pressure. iPTH was correlated with systolic $(\mathrm{r}=0.265, \mathrm{p}=$ $0.000)$ and diastolic blood pressure $(r=0.216, p=0.001)$ in whole sample. However, in individuals homozygous ff genotypes, the vitamin D was correlated with systolic $(\mathrm{r}=0.283, \mathrm{p}=0.007)$ and diastolic blood pressure $(r=0.293, p=0.005)$. Individuals heterozygous $\mathrm{Bb}$, vitamin $\mathrm{D}$ was correlated with diastolic blood pressure $(\mathrm{r}=0.200, \mathrm{p}=$ 0.047). Previous data suggests that VDR polymorphisms could participate of elevation of blood pressure, increasing the susceptibility of hypertension. In our study, the relationship between vitamin D and blood pressure only appear in individuals homozygous ff genotype and in individuals heterozygous $\mathrm{Bb}$ genotype, showing the possible influence that polymorphisms could exert in hypertension. 
P236

\section{Physico-Chemical Characterization of Yacon}

Wanise Maria S. Cruz, Shizuko Kajishima, Matilde Pumar, Carolina Oliveira Coutinho, Sabrina Mattos Guimarães,

Natália Alvarenga Borges

Universidade Federal Fluminense

Introduction: The yacon (Polymnia sonchifolia Poepp) is a tuberous root, originated in the Andean region of South America It is considered functional food due to the high content of fructooligosaccharides (FOS), precursor of inulin, a prebiotic. The functional effects attributed to yacon are: Improves the immune response due to the stimulation of growth of bifidobacteria, better glycemic control and hyperlipidemia. FOS are fermented by the microflora in the colon, producing fatty acids and organic acids which lower the $\mathrm{pH}$ in the intestinal lumen, increasing the concentration of ionized minerals, including calcium, which favors uptake by passive diffusion (Caprilles et al. 2005).

Objectives: To physically and chemically characterize yacon, in natura.

Methods: Samples were acquired in Niteroi / RJ. Were determined $\mathrm{pH}$, titratable acidity (TA), total soluble solids ( ${ }^{\circ}$ Brix), moisture, protein, lipid and ash, according to the methods Instituto Adolfo Lutz (2005). The percentage of total carbohydrate was determined by the difference of the percentage of the sums of moisture, fat, protein and ash (USP, 2004). To determine the energy value was considering the conversion factors of Atwater (USP, 2003).

Results: The analysis results were compared with the results found in the literature, with some variations: $\mathrm{pH}$ 5.51, ATT 0.318, $1.332^{\circ}$ Brix, moisture $90.08 \%$, ash $0.33 \%, 0.05 \%$ lipids and proteins $320 \%$. ${ }^{\circ}$ Brix values and ATT were different from literature, the $\mathrm{pH}$ values were closer to those found by Ventura (2004) and Michels (2005). Despite the lack of literary data, the determination of these is important for the assessment of post-harvest quality of yacon. The value obtained was $6.34 \%$ carbohydrates, different from those found by Lachman (2003), 13.8\%, and Michels (2005), 12.5\%. This difference can be explained by variations in the composition of sugars and fructans of yacon in different harvest times and growing conditions. The energy value found was $38.6 \mathrm{kcal} / 100 \mathrm{~g}$ close to that obtained by Michels (2005), $34.7 \mathrm{kcal}$.

Conclusions: The yacon is a food low in energy. For its functional properties it is an alternative feed for prevention and treatment of various diseases nutrition.

\section{P237}

\section{Soy Protein-Based Infant Formulas and Its Adverse Effects: A Systematic Review}

\author{
Yasmin N.V. Amaral, Lucia Gomes Rodrigues \\ Universidade Federal do Estado do Rio de Janeiro
}

Cow's milk allergy is the most common food allergy in infants, because they have an immature immune system and rely heavily on antibodies in breast milk. The use of hypoallergenic formulas is considered the alternative proposed. Currently there are three types of formulas that can be indicated in the dietary treatment of allergy in infants: Soy Protein based formulas, extensively hydrolyzed casein formulas, and free amino acids based formulas. The soy formulas have high amounts of phytate, aluminum and phytoestrogens that can bring undesirable effects to child health. Therefore, the objective of this review was to describe the adverse effects of the use of soy formula in infants. This study was a systematic review by searching the electronic databases Pubmed and Biblioteca Virtual em Saúde, whose sources of information to Cochrane, SciELO, LILACS and MEDLINE. The articles were selected from the reading of the abstracts that should provide the following information: the content of phytoestrogens and / or aluminum in soy formula compared to other formulas and breast milk and its possible consequences, evaluation of growth of infants fed with soy formula and review articles were excluded. Have been created three cards from extraction of information from selected articles, first of all have been identified 210 articles, 19 were selected for full reading, and only 14 were included in the review for meeting all inclusion criteria. The articles dated from 1994 to 2012 and represented 7 countries: United States (42.9\%), Italy (14.3\%), United Kingdom (14.3\%), Spain $(7.1 \%)$, Finland (7.1\%), Canada (7.1\%) and New Zealand (7.1\%). Five studies addressed the issue of growth and development of infants fed with soy formula, five discussed the amount of phytoestrogens in soy formula and other four aluminum. As for weight of the infants, only one study reported a reduction in infants fed with soy formula. Other studies showed extremely higher amounts of isoflavones and aluminum in soy formula when compared to milk-based formula or breast milk. Studies have shown the existence of high levels of isoflavones (genistein and daidzein) and aluminum in both formulas as in infants fed with soy formula and this fact may lead to adverse effects on human health both during childhood and adulthood. 


\section{Author Index}

\section{Journal of \\ Nutrigenetics Nutrigenomics}

Numbers refer to page number
Abdalla, D.S.P. 195, 269

Acosta, D.A.V. 209

Agostinho, C. 203, 204

Ahn, J. 285, 286

Aikawa, J. 230, 282, 283

Aissa, A.F. 287

Alberici, L.C. 191, 262

Albuquerque, K.T. 216

Albuquerque, L.L. 232

Alcadipa, F.C. 217

Alencar, L.L. 250

Alencar, N.M.N. 284

Alezandro, M.R. 253

Allayee, H. 182

Aller, E. 233

Almeida, C.G. 214

Almeida, D.C. 278, 294

Almeida, D.F. 217, 281

Almeida, I.S. 205

Almeida, M.G. 227

Almeida, M.R. 247

Almeida, S. 260

Alonso-Vale, M.I.C. 258, 262, 267

Alves, C.X. 249

Alves, H.H. 235

Alves, R.D.M. 288

Alves, S.R. 206

Amano, M.T. 294

Amaral, C.L. 196, 198, 283

Amaral, Y.N.V. 297

Amorin, L.L. 244, 260, 261

Andrade, F.O. 240

Andrade, I.S. 266

Andrade-Oliveira, V. 294

Andrade, V. 201

Angelis-Pereira, M.C. 197

Antoine, Jean-M. 191

Antunes, L.M.G. 247, 287

Aprile, S. 263

Aragão, K.S. 284

Arantes, V.C. 263, 294

Araujo, G.L. 255, 256

Araújo, T.M.C. 195

Arçari, D.P. 221

Arifa, R.D. 244

Arlorio, M. 263

Arthur, T. 291

Ashino, N.G. 202, 274

Assis, R.C.L. 234

Avila, D.S. 256, 257

Azevedo, M.C. 277
Bajay, L.G. 274

Baliane, T.S. 292

Baltar, V.T. 252

Bamberg, D. 289, 290

Banin, R.M. 266

Barale, R. 192

Barbosa, A.C.S. 227

Barbosa, D.S. 218

Barbosa, J.G. 248

Barbosa, K.B.F. 198, 239

Barbosa, M.O. 255, 265

Barbosa, M.R. 191, 262

Barquilha, G. 213

Barrett, K.E. 190

Barros, C.R. 252, 266

Barros, F.S. 231

Barros, N.M. 286

Barros, N.V.A. 245

Barros, T.A. 245, 246, 247

Barroso, C.F. 272

Bassante, F.E.M. 228

Bassante, F.M. 199, 211, 236, 257

Bassi, E. 201, 294

Bastos, A.Q.A. 278

Batista, Â.G. 274

Batista, D.F. 203

Batista, K.J.R. 248

Batista, L.P.R. 242, 243, 245 , 246, 247, 248, 293

Batista, L.R. 242, 243, 245, 246, 247, 248

Batista, M.A. 244, 260, 261

Batista, T.M. 203

Bechara, L.R.G. 251

Beger, R. 180

Belinetti, F.M. 219

Bellone, S. 263

Beltra, J.S.O. 280

Beltran, J.O.S. 232

Beltran, J.S.O. 237, 280

Benatti, R.O. 202, 274

Benevides, F.T. 284

Bento-Santos, A. 223

Berndt, S.I. 243

Berti, A.P. 231

Bezerra, A.P.M. 222

Bezerra, J.F. 227

Bianchi, M.L. 247

Bianco, B. 217

Bispo, K.P. 216

Bispo, M. 249
Blehm, C. 289, 290

Boasquivis, P. 281

Boer, P.A. 270, 271

Bogle, M. 180

Bolaños-Jiménez, F. 187, 276

Bollati, M. 190

Bona, G. 263

Bonatto, S. 230

Bonatto, S.J.R. 282

Bonomo, L.F. 281

Bordin, S. 206, 249, 264, 267

Borelli, P. 232, 237, 262, 267, 280, 290

Borges, A.C. 246

Borges, M.C. 262, 267, 290

Borges, N.A. 297

Borges-Silva, C.N. 215

Borges, T. 229

Borghetti, G. 230, 282

Boroni, A.P. 288

Bortolin, R.H. 227

Boschero, A.C. 237

Botelho, A.P. 275

Bouts, D.M.D. 222

Bowman, F. 233

Braga, M. 265

Brand, C.C. 237

Brandao-Neto, J. 249

Braz, J.R.C. 279

Braz, L.G. 279

Braz, M.G. 279

Bressan, J. 198, 214, 234, 239, 240, 288

Brito, G. 230

Britto, J.C. 268

Brosnan, J.T. 273

Brosnan, M.E. 273

Brum, P.C. 251

Bukhari, A. 194

Burgueño, A.L. 193

Burim, R.V. 247

Cabe-Sellers, B.Mc, 180

Caçula, K.G. 235

Cadioli, M.G.B. 258

Calabriso, N. 225, 226

Caldas, D.R.C. 193

Câmara, D.F. 256, 257

Câmara, N. 187, 201, 210, 275 , 294

Camargo, J.A. 233

Camargo, V.B. 199
Campa, D. 192

Campagnolo, P.D.B. 260

Campión, J. 234, 236

Campos, A. 241

Campos, G.D.O. 260

Campos, N.C. 244, 260, 261

Cândido, A.P.C. 277, 278

Canzian, F. 192

Caperuto, L. 249

Carabelli, B. 232

Cardoso, J.F.R. 212

Cardoso, K.B. 294

Cardoso, L.D. 269

Cardozo, M.T. 282

Caricilli, A. 201

Carli, A.P. 294

Carlos, A.C.C.C. 197,259

Carluccio, M.A. 225, 226

Carmo, M.C.N.S. 256

Carneiro, E.M. 191, 203, 237, 263

Carnelossi, M.S. 218

Carpinelli, A.R. 283

Carrai, M. 192

Carraro, J.C.C. 240

Carvalheira, J.B. 233, 293

Carvalho, F.M.C. 195

Carvalho, J.A.S. 245, 246, 247

Carvalho, M.H.B. 268

Carvalho, R.F. 241, 271

Casey, J. 190

Castiglioni, S.A. 266

Castoldi, A. 201, 294

Castro-Barbosa, T. 249

Castro, I.M. 277, 278

Castro, L.L. 292, 293

Castro, L.M.S. 272

Cavalcante, M.F. 269

Cechinel-Filho, V. 236

Cenedeze, M.A. 275

Cerda, A. 287

Cerqueira, J.J. 270, 271

Chambaud, I. 190

Champagne, C. 180

Chango, A. 251

Chiang, N. 184

Chimin, P. 258

Cicogna, A.C. 200

Cintra, D.E. 293

Clarke, S.E. 219

Clemente, A.P.G. 266

Coelho, I. 230, 282

\section{KARGER}

(C) 2012 S. Karger AG, Basel

Fax +41613061234

E-Mail karger@karger.ch

www.karger.com

Accessible online at:

www.karger.com/jnn 
Coelho, L.M. 231

Cogliati, B. 249

Cohen, C. 212

Coimbra, C.C. 241

Colpo, A.C. 199, 211, 228, 236, 257

Conceição, L.L. 216, 246, 288

Constantin, P.C. 289, 290

Conti, A. 281

Cookson, A.L. 212

Coope, A. 294

Cordeiro, H.S. 277

Cordero, P. 236

Correa-Costa, M. 294

Correa, C.R. 200, 227, 276

Corrêa, M.N. 207, 208, 209, 272

Costa, N.M.B. 269

Costa, P.S. 204

Cota, R.Guerra-S. 241

Coutinho, C.O. 297

Cozzolino, S.M.F. 205, 250

Crisma, A.R. 198, 223, 245, 283

Crocco, P. 192

Cruz, K.J.C. 193, 225, 242, 243, 245, 246, 247, 248, 292 , 293

Cruz, W.M.S. 297

Cruzat, V.F. 295

Cuevas, A. 195, 269

Cunha, M.C.R. 280

Curi, R. 183, 191, 193, 194, 196, 198, 201, 206, 210, 213, 223, 224, 230, 235, 238, 240, $245,251,255,258,264,265$, $267,275,283,286,294,295$, 296

Curi, T.C.P. 235

Cursino, J. 202

Curti, M.L.R. 252, 266

Cury-Boaventura, M.F. 206, 235

Cvoro, A. 260

Cyrino, E.S. 218

Czarnobay, S.A. 255

da Conceição, L.L. 270

da Costa, C.E.M. 232

da Rocha, T.J. 289, 290

Da Rosa, F.T. 207, 208

da Silva Aragão, R. 187, 276

da Silva Brito, A.K. 193, 225

da Silva Fraire, P. 231

da Silva, L.E. 248

Da Silva, L.L. 270

da Silva Marineli, R. 213, 274, 275

Da Silva, S. 201

da Silveira Alves, V. 255
Dagli, M.L. 240, 249

Damasceno, N.R.T. 287

Daniel, H. 188, 238

Darin, J.D.A.C. 247, 287

das Neves, R.X. 223, 224

de Abreu, I.C.M.E. 253

de Almeida Amaral, E.E. 222

de Almeida Coelho, C. 209

de Andrade, F.M. 289, 290

de Angelis-Pereira, M.C. 197, 259

de Araújo Silva, T.L. 273

de Azevedo, M.C. 254

de Barros, M. 282

de Barros Mucci, D. 216

De Barros Reis, M.A. 263

de Brito Rodas, M.A. 258

de Camargo, V.B. 236

de Carli, E. 224

de Carvalho, Á.C.S. 193, 225

de Carvalho, V.B.L. 193, 225

de Cássia Borges Castro,

R. 217, 281

de Cassia dos Santos, A. 292

de Castro Marcondes, J.P. 278

de Castro, R.M. 187, 273

De-Castro, R.Manhães-, 276

De Caterina, R. 181, 225, 226

de Conti, A. 240, 241

De Faria, T.S.M. 258

de Fátima Paiva Baracho, M. 249

de Fátima Rodrigues, C. 224

de Fátima Silva Ramalho,

A. 263

de J. Hernandez-Vazquez,

$$
\text { A. } 226
$$

de Jesus, G.P.P. 228

de Lima, D.C. 241

de Lima Junior, E.A. 223, 224

de Lima Reis, S.R. 263

de Lima, T.M. 238

de Lima, W.T. 210

de Lourde Pires Bianchi,

$$
\text { M. } 287
$$

de M. Barros, H.R. 235

de Matos, R.J.B. 273

de Melo Loureiro, A.P. 240

de Meneses Fujii, T.M. 267, 290

de Miranda, J.X. 240

de Moraes Honorato de Souza, G.I. 264

de Moura, C.F.G. 228

de Oliveira, L.G.F. 222

de Oliveira, E.F. 203, 204

de Oliveira, T.B. 240

de Oliveira, T.F. 240

de Paula, H. 269

de Paula Oliveira, R. 230 de Paula Porto, M. 278

De Paula, S.O. 267

De Rango, F. 192

de Sousa, L.G. 196, 251, 255

de Sousa Santos, A.N. 248

De Sousa Silva Rios, G. 193, 225

de Souza, L.A. 222

de Souza, L.E. 194

De Souza Meirelles, M.S. 212

de Souza, T. 201

Delboni, F.R. 217

Deminice, R. 229, 248, 273

Dennis, E.A. 184

Deocleciano, O.B. 284

Deram, S. 291

Desjardins, Y. 202

de $\sim$ Souza $\sim$ Jr. A.L. 194

Dias, M.M. 216

Diaz-Ruiz, R. 226

Dichi, I. 218, 219

Diniz, M.T.C. 285

Diniz, S. 291

Dionello, N.J.L. 208, 272

Dior, A. 251

do Amaral, C.L. 223, 245

do N. Marreiro, D. 193, 225

Domenici, F.A. 212

Domiciano, M. 249, 264

Dorea, E.L. 287

Dornelles, P. 290

dos Reis Silveira, L. 262

dos Santos Garbim, T.H. 231

dos Santos Gomes, L. 222

Draper, C. 179

Dubois, M.J. 202

Dullius, J. 206

Durda, K. 284

Durso, D.F. 256

Dyer, J. 212

Esposito, D. 220, 284

Esposito, E. 264

Evangelista, E.A. 241

Factori, R. 232

Fagundes, C.T. 183

Fagundez, D. 256, 257

Fajardo, C.M. 287

Faria, F.R. 271

Farias, T. 296

Farjado, C.M. 249

Felizardo, R.J.F. 275

Ferguson, L.R. 186

Fernandes, A.A.H. 203

Fernandes, L.C. 230, 282, 283

Fernandez, T. 190

Ferraz, L.F.C. 221

Ferreira, A.B. 246

Ferreira, A.L.A. 200, 276
Ferreira, A.L.D.A. 279

Ferreira, A.R. 241

Ferreira, A.V. 220, 244, 261, 285

Ferreira, C.M. 210, 294

Ferreira, L.G. 255

Ferreira, S.R.G. 252, 266, 296

Festuccia, W. 186, 235, 240, 245, 253

Festuccia, W.T. 196, 206, 238, 258, 295, 296

Fialho, E. 204, 222, 254, 277

Fiamoncini, J. 235, 238, 283

Fiegenbaum, M. 289, 290

Figueiredo, I.F.T. 284

Filho, A.A. 268

Fock, R.A. 262, 267, 280, 290

Folador, A. 283

Folchetti, L.D. 266

Folmer, V. 199, 228, 236, 257

Fonseca, A.C.M. 216, 270

Fonseca-Alaniz, M.H. 262, 267

Fonseca, T.L.R. 289, 290

Foss, N.T. 199

Frade, M.A. 199

Franceschini, S.D.C.C. 271

Francisco, L.V. 229, 248

Francisqueti, F.V. 227

Frazzatto, E. 291

Freire-Neto, F.P. 227

Freitas, B.J.S.A. 292, 293

Freitas, F.S. 246

Freitas, R.N. 277, 278

Fujii, T.M. 262

Fukushima, J.I. 282

Furlan, C.P.B. 213, 214, 274, 275

Furtado, K.S. 241

Gaiolla, P.S.A. 203

Galvão, B.C.O. 195

Garbelotti, M.L. 258

García, G.P. 187

Garcia, S.B. 212

Garcia, V.C. 268, 296

Garcia, Z.M. 244

Garofolo, I.C. 206

Genovés, S. 190

Genovese, M.I. 202, 206, 235, 253, 264

Gern, B.H. 282

Giano, T.F. 193

Giusti, K.C.S. 268

Godinho, A.F. 271

Gollucke, A.P.B. 228

Gomes, T.D.U.H. 287

Gonçalves, A.E.S. 202, 206

Goncalves-Santo, C.C. 215

Gontijo, J.A. 228, 229, 270, 271

Gontijo, L.N. 216 
Gorjao, R. 206, 213, 215, 230, $235,238,265,283$

Gorofolo, I.C. 264

Green, B. 180

Grompone, G. 190

Grosa, G. 263

Guaita, G.O. 231

Guazzelli, I. 291

Guedes, A. 289

Guerra, B.A. 203

Guerra, J.C. 230

Guerra, J.F. 239, 253, 281

Guerra-Shinohara, E.M. 268

Guido, L.N. 251

Guimarães, J.B. 241

Guimarães, J.C. 237

Guimarães, S.M. 297

Guirado-Rodrigu, S.H. 215

Gupta, S. 284

Guzmán-Quevedo, O. 187, 276

Gwon, S.Y. 285

Ha, T. 285,286

Haag, A. 238

Hachul, A.C.L. 264

Hannah, S.S. 238

Hashimoto, N.N. 250

Hatanaka, E. 183, 230, 265

Henriques, A. 241

Hermsdorff, H.H.M. 234

Hernandes, L.C. 247

Hernández, A. 196

Hipólito, T.L.B. 292, 293

Hirabara, S. 213

Hirabara, S.M. 196, 223, 224, $235,238,245,251,283$

Hirata, B.K.S. 266

Hirata, M.H. 227, 249, 287

Hirata, R.D.C. 182, 227, 287

Hiyane, M.I. 294

Hodgkinson, A.J. 212

Holway, H. 220

Huang, T. 192

Huang, Wen-Y. 243

Ignacio-Souza, L.M. 202, 274, 294

Imaizumi, E. 276

Ip, B. 201

Irigoyen, M.C. 194

Itoh, K. 212

Jacob, P.S. 262, 267, 290

Jacobs, R.L. 273

Jacometo, C.B. 207, 208, 209, 272

Jakubowska, A. 284

Jasper, C.H. 282

Jaworska, K. 284

Jenkins, G. 190
Jerônimo, S.M.B. 205

Jordao, A.A. 229, 273

Jordão Jr. A.A. 248

Jorge, M.I.E. 287

Jún, P.I.H.B. 295

Jung, C.H. 285, 286

Junio, C.F.R. 223

Junior, A.J. 199

Junior, A.O. 289, 290

Junior, I.R. 278

Júnior, M.R.M. 213, 214

Júnior, S.J.D. 255

Kajishima, S. 297

Kang, J.X. 188, 189, 219, 286

Kaput, J. 180, 190

Katashima, C.K. 233, 293

Katki, H.A. 243

Katz, I.S.S. 232

Keesen, T. 205

Kettelhut, I. 191, 262

Kiyokawa, R.K. 287

Komarnytsky, S. 220, 284

Korb, C. 289, 290

Kroll, C. 255

Ladeira, M.S.P. 279

Ladim, K.C. 206

Laitinen, K. 288

Lajolo, F. 235

Lajolo, F.M. 202, 206, 253, 264

Lambertucci, R.H. 283

Lana, J.P. 220

Latorraca, M.Q. 263, 294

Leal, C.B. 293

Leal, L.R. 293

Leal, L.T. 258

Leandro, C.G. 223

Leandro, E.S. 246

Lee, H. 286

Leite, J.A. 256

Leite, J.I.A. 285

Leite, L.D. 249

Leite, T.M. 277

Leles, S.M. 231

Lellis-Santos, C. 206, 249, 264

Lemes, S.F. 263

Lenhare, L. 293

Lenquiste, S.A. 274, 275

Levada-Pires, A.C. 193, 206

Li, D. 192

Li, J. 238

Libra, G.N.M. 232

Lima, E. 251

Lima, F. 280

Lima, F.B. 258

Lima, M.E. 199, 211, 228, 236, 256, 257

Lima, R.L. 244

Lima-Salgado, T.M. 193
Lima, S.M.T. 242, 243, 245 , 246, 247, 248, 292, 293

Lins, É.M. 293

Lipay, M.V. 217

Loor, J.J. 207, 208

Lopes, A.B. 240, 258

Lopes, A.S. 270, 271

Loureiro, M.B. 227

Lozovoy, M.A.B. 219

Lubinski, J. 284

Luglio, H.F. 233

Luiz, R.S. 249

Luperini, B.C.O. 278

Lustosa, B.H.B. 203

Luvizotto, R.A.M. 200, 201, 227,276

Ma, D.W.L. 219

Macedo, V.S. 288

Machado, A.B.F. 270

Machado, A.M. 269

Machado-Coelho, G.L.L. 277, 278

Maciel, B.L.L. 205

Madeira, S.V.F. 284

Magalhães, G.A. 244

Magdalon, J. 183, 240, 295, 296

Maia, C.S.C. 272

Malek-Zadeh, C.H. 210

Malfitano, C. 194

Mancini, D.A.P. 199

Mancini-Filho, J. 199

Mansil, N.L.G. 193

Marchini, J.S. 210

Marcotte, B. 202

Mardiana, M. 194

Marette, A. 188, 202, 253, 296

Marian, G.A.A. 266

Marian, R.B. 289

Mariman, E. 233

Marin, B.K. 263

Marin, L. 263

Marineli, R.S. 214

Marniar, M. 194

Maróstica-Júnior, M.R. 274, 275

Marques, A.C. 214, 274

Marques, A.Y.C. 213

Marques, C.G. 206

Marques, P.E. 220

Marreiro, D.N. 242, 243

Martin, R.L. 180

Martinez, C.S. 211, 236, 257

Martinez, J.A. 188, 234, 236

Martini, L.A. 268, 296

Martins, A.O. 200

Martins, A.R. 196, 198, 245, 283

Martins, L.B. 244
Martins, L.M. 242

Martins, M.D.C.C. 292, 293

Martorell, P. 190

Masi, L.N. 198, 245, 283

Masini-Repiso, A.M. 211

Massaro, M. 225, 226

Mastroeni, M.F. 255

Mastroeni, S.S.B.S. 255

Mathers, J.C. 185

Matsubara, L. 203

Mattei, P. 208, 209, 272

Mattevi, V.S. 260

Mazzotti, T.K.F. 217

Mello, A.S. 280

Melo, A.M. 202, 274

Melo, I.L.P. 199

Melo, P.A.S. 219

Melo, S.S. 282

Melo, T.R. 227

Mendes, A. 199

Mendes, E. 210

Mendes, F.A.V. 232

Mendes, M.C. 233

Mendez, A.L. 236

Menezes-Garcia, Z. 261

Menezes, G.B. 220

Menezes, J.G.K. 249

Mercadante, A.Z. 247

Mesquita, F. 228, 229

Micheletti, T.O. 233, 292, 293

Milagro, F.I. 234, 236

Milanski, M. 294

Minicucci, M. 203

Miotto, C.H. 207

Mir, C.G. 228

Miranda, N.C.M. 276

Moia, S. 263

Moisa, S. 207

Momesso, C.M. 213, 235

Monfort-Pires, M. 266

Monteiro, J.P. 180, 209

Monteiro, N.S. 268

Montesanto, A. 192

Moore, L.E. 243

Moraes, C.A. 246

Moraes, L.F.S. 267

Moraes, L.N. 271

Moraes, T.I. 287

Moraes-Vieira, P.M. 294

Morais, A.H.A. 195

Morais, L.V. 227

Morari, J. 293

Moreno, F.S. 240, 241, 281

Moreno, M.F. 264

Mori, M.A. 203

Morine, M. 180

Moriscot, A. 191

Mota, M.S. 286

Moura, R.S. 237

Mourão, D.M. 198, 239 
Mulet, A.P. 190

Müller, C. 198, 239

Muller, E.S. 270

Munhoz, A.C. 232

Murata, G.M. 230, 265

Muszynska, M. 284

Nabb, W.C.Mc, 212

Nachbar, R.T. 223

Nascimento, A.F. 200, 201, 227 , 276

Nascimento, C.M.O. 266

Nascimento, L.M. 193, 225

Nascimento, N.N. 243

Navarro, A.M. 199

Nazario, A.P. 289

Neto, F.P.F. 205

Neto, J.C.R. 223, 224, 245, 251

Neto, J.F.R. 205

Nicola, J.P. 211

Nicoletti, C.F. 210

Nogueira, C.R. 276

Nonino, C.B. 210

Norde, M. 262, 267

Novais, P.F.S. 278

Nunes, A.C.S. 206

Nunes, M.T. 211, 249

Ochoa-Ruiz, E. 226

Okino, A.M. 218

Okoshi, K. 200, 203

Okuda, M.H. 264

Oliveira, A.C. 275

Oliveira, A.M.L. 227

Oliveira, A.R.S. 242

Oliveira, D.C. 237,280

Oliveira, D.M. 222

Oliveira, F.M. 199

Oliveira, H.C.F. 235, 237

Oliveira, K. 217

Oliveira, M.C. 244, 261, 285

Oliveira, M.N. 246

Oliveira, R. 287

Oliveira, R.P. 281

Oliveira, T.B. 295, 296

Oliveira, V.A. 275

Oliveira, Y.M. 227

Oliverira, T.A.S. 200

Ong, T. 179, 185, 240, 241, 250,281

Origassa, C. 275, 294

Ortega-Cuellar, D. 226

Osorio, J. 207

Ostrowski, M. 269

Otter, D.E. 212

Oyama, L.M. 264, 266

Ozanne, S. 185

Pacheco, A.C.L. 222

Pacheco-Silva, A. 275
Paiva, A.A. $245,247,248$

Paiva, F.A. 230

Palioto, G.F. 231, 232

Pantaleão, L.C. 206, 249, 267 , 290, 295

Pardo, L.B. 275

Paschoal, V.A. 240, 295, 296

Passarino, G. 192

Patrício, P.R. 235

Pauli, J.R. 293

Pedrosa, M.L. 230, 239, 253, 281

Pedrote, P.M. 222

Pêgo, J.M. 270, 271

Pellegrino, M. 226

Peluzio, M.D.C.G. 216, 246, 267, 270, 271, 288

Penalva, S. 280

Pence, L. 180

Pequito, D. 230, 282

Percival, S.S. 199

Pereira, P.F. 271

Pereira, R.L. 275

Pereira, R.W. 277

Pereira, S.S. 256,285

Pérez, E.M.S. 284

Perez-Garcia, G. 276

Peron, J.S. 294

Perrone, C.E. 203

Pérusse, L. 189

Pessoa, P.P. 272

Pfeifer, L.F.M. 208, 272

Pierine, D.T. 276

Pimentel, G.D. 233, 292, 293

Pinheiro, C.H. 255

Pinheiro, C.H.J. 223

Pinho, M.C. 273

Pinho, V. 261

Pino, F.A.B.D. 208, 209

Pino, F.B.D. 272

Pires, M.M. 252

Pirola, C.J. 193

Pithon-Curi, T. 193, 206, 213, 215, 223, 251, 283

Poetini, M.R. 211

Polato, T.H.P. 215

Polinati, R.M. 254, 277

Portela, J.V.F. 293

Pozza, F.S. 275

Prada, P.O. 292, 293

Prado, R.P. 278, 279

Prasad, C. 179, 181

Presti, P.T. 276

Priami, C. 180

Priore, S.E. 271

Prodam, F. 263

Pumar, M. 297

Purdue, M.P. 243

Quadros, D.E. 217
Quaresma, P.G.F. 292

Queiroz, C. 254, 277

Queiroz, E.M. 277, 278

Queiroz, K.B. 241

Rabelo, L.M.A. 195

Radiah, R. 194

Ramalho, M. 292

Ramon, D. 190

Ramos, P.S. 204

Raposo, H.F. 235

Ravacci, G.R. 217, 281

Reencober, N. 292

Reis, B.Z. 205

Reis, C.E.G. 206

Reis, M.A.B. 294

Reis, S.A. 216

Reis, S.M.P.M. 275

Reis, S.R.L. 294

Repka, J. 282

Rezende, A.A. 227

Rezende, L.A. 227

Ribeiro, A.Q. 267

Ribeiro, C.C.S. 266

Ribeiro, D.A. 228

Ribeiro, E.B. 266

Ribeiro, F.A.P. 228

Ribeiro, M.L. 221

Ribeiro, O.G. 232

Ribeiro, R.S. 276

Rizzato, C. 192

Roccio, M. 263

Rocha, A.L.S. 285

Rocha, A.P.M. 237

Rocha, C.L.M.S.C. 231, 232

Rocha, G.Z. 233, 293

Rocha, J.L.M. 198, 239

Rocha, M.S. 283

Rodovalho, G.V. 241

Rodrigues, A.C. 196, 223, 251, 283

Rodrigues, A.J. 270, 271

Rodrigues, A.L.S. 255

Rodrigues, A.N. 217

Rodrigues, D.F. 220

Rodrigues, E. 247

Rodrigues, H.G. 183

Rodrigues Jr. C.F. 251, 283

Rodrigues, L.G. 217, 276, 297

Rodrigues, L.O. 216

Rodrigues, M.E. 216

Rodrigues, R.O. 260

Rodrigues, T.N. 286

Roela, R.A. 217

Rogero, M.M. 252, 262, 267, 290

Rohani, R. 194

Romanatto, T. 238, 286

Ronque, E.R.V. 218

Ropelle, E.R. 233, 293
Roquim, M. 206

Rosa, D.D. 216

Rosa, F.T. 229

Rosa, H. 199, 211, 228, 236, 257

Rose, G. 192

Rowe, C.A. 199

Roy, N.C. 212

Ruas, R.N. 256

Rudnicki, M. 195

Saad, M. 201

Saad, M.J. 233, 292, 293

Saavedra, N. 195, 269

Sabóia, L.V. 282

Salazar, L.A. 195, 196, 269

Salgado-Filho, W. 210

Salgueiro, W.G. 256, 257

Salminen, S. 288

Salvador, E.P. 266

Salvadori, D.M.F. 278, 279

Salvanha, D.M. 222

Sampaio, F.A. 243

Sampaio, I. 191, 262

Santos, A. 213

Santos, A.F.F. 272

Santos, A.M. 277

Santos, A.N.S. 245,248

Santos, B. 264

Santos, B.F. 279

Santos, C.M.M. 215

Santos, E.A. 195

Santos, E.W. 237, 280

Santos, F.M. 227

Santos, G.A. 202

Santos, J.C. 221

Santos, L.B. 272

Santos, L.R. 206

Santos, M.F.A. 254

Santos, M.G.N. 249

Santos, R.A.D. 212

Santos, V.C. 206

Santos-Zago, L.F. 275

Sardinha, F.L.C. 216

Scheler, H. 218

Schelles, C. 249

Schmidt, B. 203, 204

Schmitt, E. 207, 208, 209, 272

Schneider, A. 208, 272

Schuch, N.J. 268, 296

Scoditti, E. 225, 226

Scolastici, C. 241

Scotti, M. 180

Scripes, N.A. 219

Sediyama, C.M.N.O. 267

Seelaender, M.C.L. 223, 224

Segantine, A.P.D. 266

Sena, C.S. 249

Sene, L.B. 271

Serhan, C.N. 184 
Serquiz, A.C. 195

Serrano-Nascimento, C. 211

Shelby, B. 180

Sieglaff, D. 260

Silva, A.M.O. 199

Silva, D.R.P. 218

Silva, F.Y. 210

Silva, G.B. 205, 232, 237

Silva, H.B.F. 294

Silva, H.P. 227

Silva, J.L. 204, 277

Silva, L.E.C.M. 229

Silva, L.L. 216

Silva, M. 239, 253

Silva, M.E. 239, 253, 281

Silva, M.M. 222

Silva-Santiago, S.C. 222

Silva, V.R. 233, 293

Silveira, L.R. 191

Silvestre, M.P. 244, 260, 261

Simão, A.N.C. 218, 219

Simão, T.N.C. 219

Simões, M.C. 235

Simopoulos, A.P. 181

Siqueira-Catani, A. 252, 266

Siqueira, N.P. 216, 270

Soares, A.T.G. 256, 257

Soares, J.C.M. 211

Soares, N.P.C. 237

Soares, R.G.P. 260

Solis, R.O. 273

Sookoian, S.C. 193

Soriani, F.M. 261

Sousa, A.F. 293

Sousa, J.M.A. 195

Sousa, M.C. 287
Sousa, M.S.B. 197, 259

Sousa, N. 270, 271

Souza, D.G. 183, 244, 261

Souza, É.S. 216

Souza, J.C. 237

Souza, K.S. 227

Souza, L.P. 250

Souza, S.L. 273

Spessoto, A.M. 265

Stanilka, J.M. 199

Storelli, C. 225, 226

Su, L.J. 243

Sun, J. 192

Tafuri, N.F. 270

Tagliati, C.A. 256

Tebas-Falcão, F. 223

Teixeira, J.M. 269

Teixeira, L.G. 285

Teixeira, M.M. 183, 210, 220, 244, 261

Teixeira, P.S. 223, 255

Teixieira, T.F. 246,118

Telles, M.M. 266

Teodoro, A.J. 237

Thum, C. 212

Tirapegui, J. 262, 267, 290, 295

Tiscornia, I. 190

Tisser, L.A. 289, 290

Togashi, M. 260

Tomeleri, C.M. 218

Tomita, L.Y. 254

Torralbo, D.F. 217

Torres, A.G.J. 281

Torres, D.B. 270, 271

Torres-Leal, F.L. 262, 267
Torsoni, A.S. 202, 274

Torsoni, M.A. 202, 274

Turner, J. 180

Uribe-Carvajal, S. 226

Ururahy, M.A.G. 227

Vale, S.H.L. 249

Valente, M.A.S. 255, 256, 265

Van Baak, M. 233

van den Berg, R. 190

Vannucchi, H. 212

Vanzela, E.C. 237, 263

Vasconcelos, D. 196, 223, 255

Vasconcelos, E.J.R. 222

Veiga, C.B. 213

Velazquez-Arellano, A. 226

Velloso, L.A. 187, 202, 274, 293

Vêncio, R.Z.N. 222

Venturini, D. 218, 219

Vieira, A.M. 183

Vieira, E.E.S. 193, 225

Vieira, F.M. 271

Vieira, L.Q. 244

Vieira, M.O. 245, 246, 247

Vieira, P.A.F. 255, 256, 265, 270

Vieira, P.M.F. 273

Vieira, T.M.F.S. 251

Villares, S. 291

Villarim, A.L.S.C. 195

Vinolo, M.A. 183, 196, 201, 275, 294

Vinolo, M.R. 198, 255, 260

Vivolo, S.R.F. 268
Voll, J.C. 231, 232

Volp, A.C.P. 198, 239

Wainwright, L.J. 190

Waitzberg, D.L. 217, 281

Walker, G.E. 263

Wang, B. 286

Wang, X.D. 200, 201

Webb, P. 260

Weissmann, L. 292

White, P.J. 188

Williams, A. 180

Wilson, M. 284

Wise, C. 180

Wollenhaupt, S.G. 256

Yamada, M. 262, 267, 290

Yamaguchi, A. 230, 282

Yamazaki, R. 230

Yamazaki, R.K. 283

Yegnasubramania, S. 243

Yeum, K.J. 276, 279

Young, W. 212

Zaltman, C. 286

Zambonatto, R. 193

Zandoná, M.R. 260

Zemdegs, J.C.S. 266

Zingali, R.B. 222

Zoppi, C. 191

Zornoff, L. 203

Zugaib, M. 268

Zulet, M.Á. 234 